\title{
More Than Meets the Eye
}

A critical semiotic analysis of landscape design visualizations

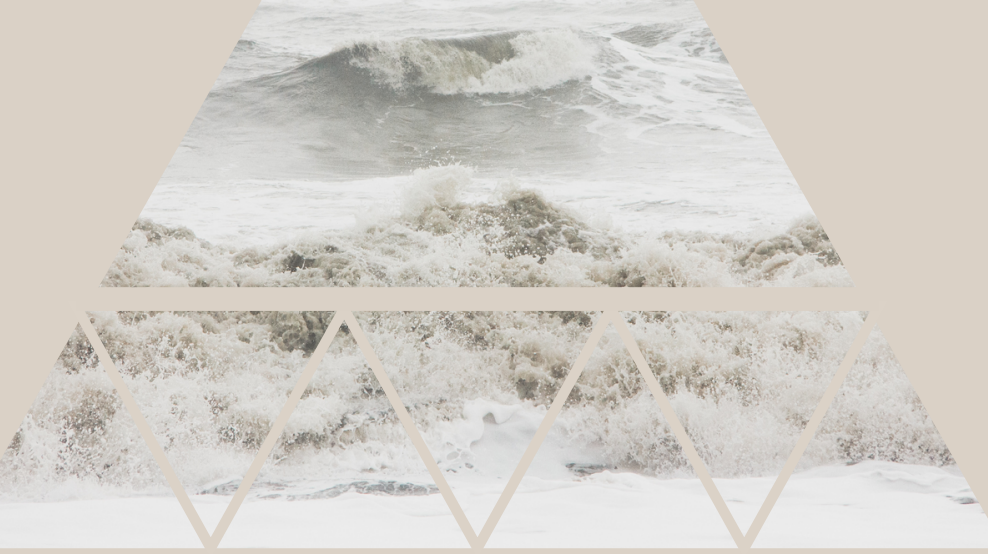

Kevin Raaphorst 



\section{More Than Meets the Eye}

A critical semiotic analysis of landscape design visualizations

Kevin Raaphorst 


\section{Thesis Committee}

\section{Promotor}

Prof. Dr A. van den Brink

Professor of Landscape Architecture

Wageningen University \& Research

\section{Co-promotor}

Dr W. van der Knaap

Assistant Professor, Land Use Planning Group

Wageningen University \& Research

\section{Other members}

Prof. Dr A.K. Bregt, Wageningen University \& Research

Prof. Dr K.J. Jørgensen, Norwegian University of Life Sciences, Ås, Norway

Prof. Dr M.N.C. Aarts, Radboud University Nijmegen

Dr R.A.J. Plant, University of Technology Sydney, Australia

This research was conducted under the auspices of the Dutch Research School of the Socio-Economic and Natural Sciences of the Environment (SENSE). 


\section{More Than Meets the Eye}

A critical semiotic analysis of landscape design visualizations

Kevin Raaphorst

Thesis

submitted in fulfilment of the requirements for the degree of doctor

at Wageningen University

by the authority of the Rector Magnificus,

Prof. Dr A.P.J. Mol,

in the presence of the

Thesis Committee appointed by the Academic Board

to be defended in public

on Tuesday 9 April 2019

at 4 p.m. in the Aula. 
Kevin Raaphorst

More than meets the eye: a critical semiotic analysis of landscape design visualizations 250 pages.

$\mathrm{PhD}$ thesis, Wageningen University, Wageningen, the Netherlands (2019)

With references, with summary in English

ISBN 978-94-6343-598-7

DOI https:// doi.org/10.18174/470766 


\section{Contents}

1 - INTRODUCTION 11

1.1 Introducing multifunctional landscapes: the Rotterdam Roof Park 11

1.2 Designing multifunctional landscapes in the face of climate change. $\quad 16$

1.3 Design visualization in landscape architecture and planning 18

1.4 Research objective and research question 23

1.5 A critical visual research approach 24

1.6 Methodology 27

1.7 Structure of this thesis 33

2 - THE SEMIOTICS OF LANDSCAPE DESIGN COMMUNICATION: towards a critical visual research approach in landscape architecture $\quad 37$

$\begin{array}{ll}\text { Abstract } & 37\end{array}$

2.1 Introduction 38

$\begin{array}{ll}2.2 \text { Semiotics } & 40\end{array}$

2.3 Iconography $\quad 45$

2.4 Simulacra and Simulation 46

2.5 'Power/Knowledge' 48

2.6 Visual Methodologies $\quad 50$

2.7 Conclusions 56

$\begin{array}{ll}\text { Acknowledgements } & 58\end{array}$

3 - READING LANDSCAPE DESIGN REPRESENTATIONS AS AN INTERPLAY OF VALIDITY, READABILITY AND INTERACTIVITY: a framework for visual content analysis $\quad 61$

$\begin{array}{ll}\text { Abstract } & 61\end{array}$

3.1 Introduction $\quad 62$

$\begin{array}{ll}\text { 3.2 Landscapes, representations and designs } & 64\end{array}$ 
3.3 A lens of semiotics and iconography 68

$\begin{array}{ll}3.4 \text { Analytic framework } & 71\end{array}$

3.5 Images, codes and interpretation $\quad 75$

3.6 Demonstrating the framework $\quad 82$

3.7 Discussion and conclusion $\quad 96$

$\begin{array}{ll}\text { Acknowledgements } & 99\end{array}$

4 - KNOWING YOUR AUDIENCE: the contingency of landscape design $\begin{array}{ll}\text { interpretations } & 103\end{array}$

$\begin{array}{ll}\text { Abstract } & 103\end{array}$

4.1 Introduction 104

4.2 Semiosis, the Interpretant, and Relational Ontologies 106

4.3 Analytical Framework 109

4.4 Rebuild by Design 113

4.5 Co-constructing Rebuild by Design 115

4.6 Discussion 128

4.7 Conclusion 131

$\begin{array}{ll}\text { Acknowledgements } & 133\end{array}$

5 - VISUALIZATION, PARTICIPATION AND RHETORIC: the discursive power of landscape design representations in participatory processes 137

$\begin{array}{ll}\text { Abstract } & 137\end{array}$

5.1 Introduction 138

5.2 Conceptual framework $\quad 140$

$\begin{array}{ll}5.3 \text { Visual discourse analysis } & 147\end{array}$

5.4 Participation, Interactivity, and Rhetoric 149

5.5 Conclusion and discussion 160

Acknowledgements 164 
6 - CONCLUSIONS AND DISCUSSION

6.1 Introduction

6.2 A critical visual research approach

6.3 How visual meaning-making processes shape participatory projects $\quad 172$

6.4 Discussion

6.5 Reflection on the research approach

6.6 Suggestions for further research

6.7 Societal relevance

References

Appendices

Summary

225

Samenvatting

Acknowledgements

About the author 



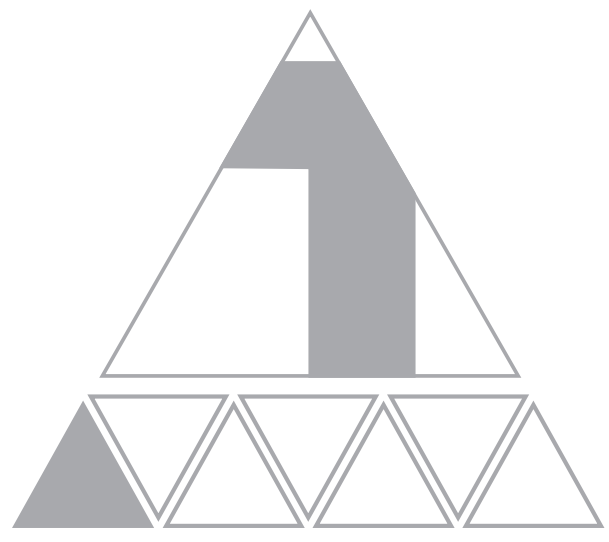





\title{
Chapter 1
}

\section{INTRODUCTION}

\author{
$x$
}

1.1 Introducing multifunctional landscapes: the Rotterdam Roof Park

The 'Bospolder' neighbourhood in the Rotterdam area of Delfshaven was physically separated from the harbour by an emplacement of twelve railroad tracks and a river dike. This created a strong barrier between the residential and industrial sites of the area (Figure 1.1). In 1998, the Dutch railway company decided that the number of tracks could be reduced from twelve to four due to a diminishing demand for rail transport (OntwikkelingsBedrijf Rotterdam 2003). This meant that fifteen acres of land became available for development; land that was highly desired by stakeholders from both the harbour area and the Bospolder neighbourhood. The ongoing discussion centred on whether or not to address the lack of green and public spaces in the neighbourhood, especially considering

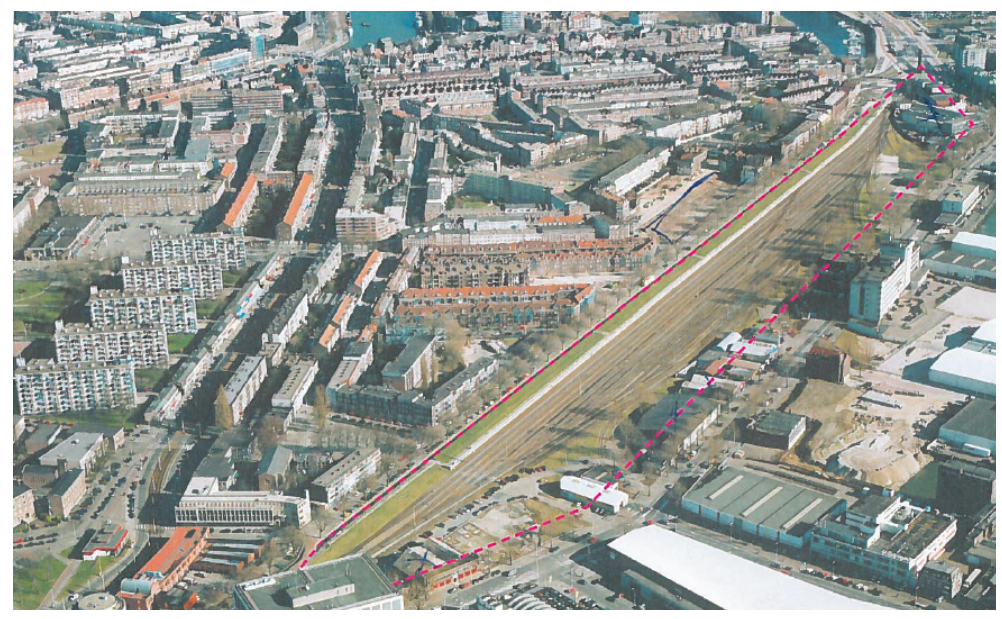

Figure 1.1. Old situation of the Rotterdam 'Roof Park': project area marked in red (OntwikkelingsBedrijf Rotterdam 2003). 
newly planned residential locations in the area. The harbour industry also made a spatial claim to the site by arguing for a much needed distribution centre and transportation hub. The connection of the site to the neighbouring river dike meant that additional requirements were posed by the water management authority that is responsible for safeguarding and enhancing flood protection in the area.

The planning process gained momentum when a city planner from the municipality of Rotterdam drew a sketch on a paper napkin in a local community centre's bar. The sketch represented the concept of an elevated park leaning onto the neighbouring river dike. This elevated park would arch over the remaining rail road tracks and cover the roofs of industrial warehouses that were to be developed at the spot of the disposable tracks. This idea (Figure 1.2) sparked interest amongst local neighbourhood inhabitants and attained public, political and financial support for the participatory planning and design process of what became known as the 'Rotterdam Dakpark' (Rotterdam Roof Park).

Design workshops were organized to gain insight into the concrete ideas and wishes of the local community. The landscape architects that facilitated these workshops used stock photos and on-the-fly photo montages to create

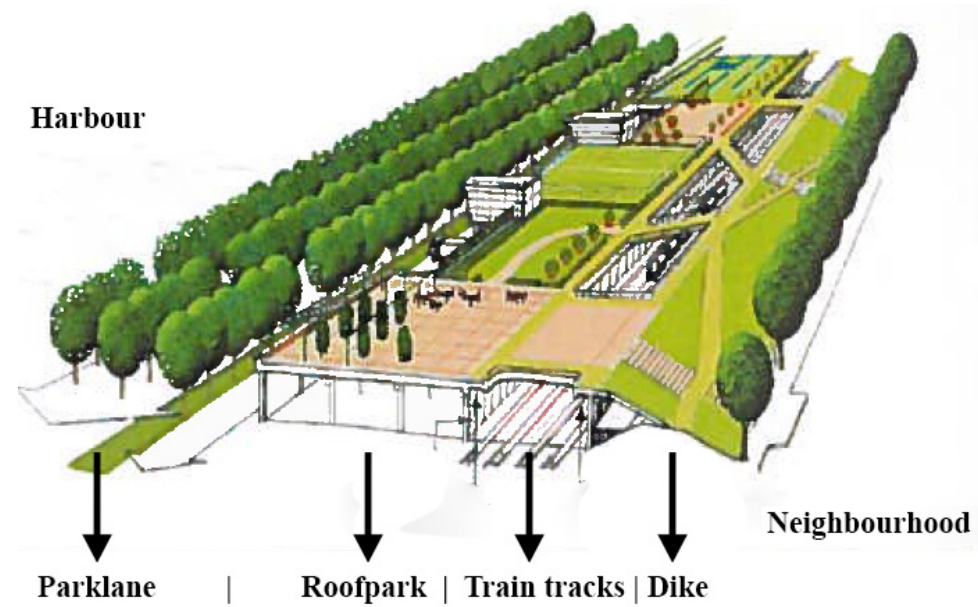

Figure 1.2. Early conceptual drawing of the Rotterdam 'Roof Park' (Adapted from OntwikkelingsBedrijf Rotterdam 2003). 
a preliminary composition of the park's architecture and together with local inhabitants developed a 'Top 10 list' of desired functions. Besides photographic techniques, physical scale models were made by the inhabitants to help them to shape their own version of the park (Figure 1.3).
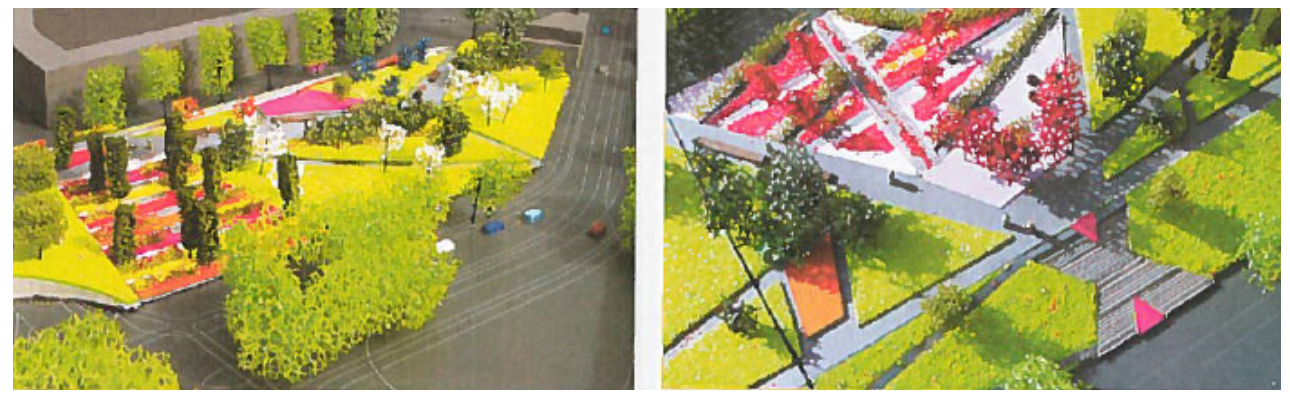

Figure 1.3. Early scale model of the Rotterdam 'Roof Park' (Ontwikkelingsbedrijf Rotterdam 2003).

Parallel to the participatory design process, the municipality of Rotterdam pursued an iconic approach: city planners envisioned a park interwoven with the city's infrastructure. The aim of this so-called 'park lane concept' was to connect several economic centres, in particular Rotterdam The Hague Airport and Delfshaven, within the metropolitan area. A bird's eye view drawing was made to depict the position of the roof park in relation to the other economic highlights of the city (Figure 1.4).

A few years into the design process, economic developments in the Rotterdam area led to a further decrease in the need to maintain industrial functions at the ground level, i.e. the rail road tracks and industrial warehouses. At this point the project developer started to advocate the commercial retail exploitation of groundlevel spaces. A realistic 3D artist impression showed their vision of the 'Bigshops Parkboulevard' (Figure 1.5).

Ultimately, the Rotterdam Roof Park boasts most of the spatial functions that came up during the planning and design process: a community park, a river dike, a shopping centre, and a green park lane that connects the various economic highlights of Rotterdam. The Roof Park project shows that, during participatory 


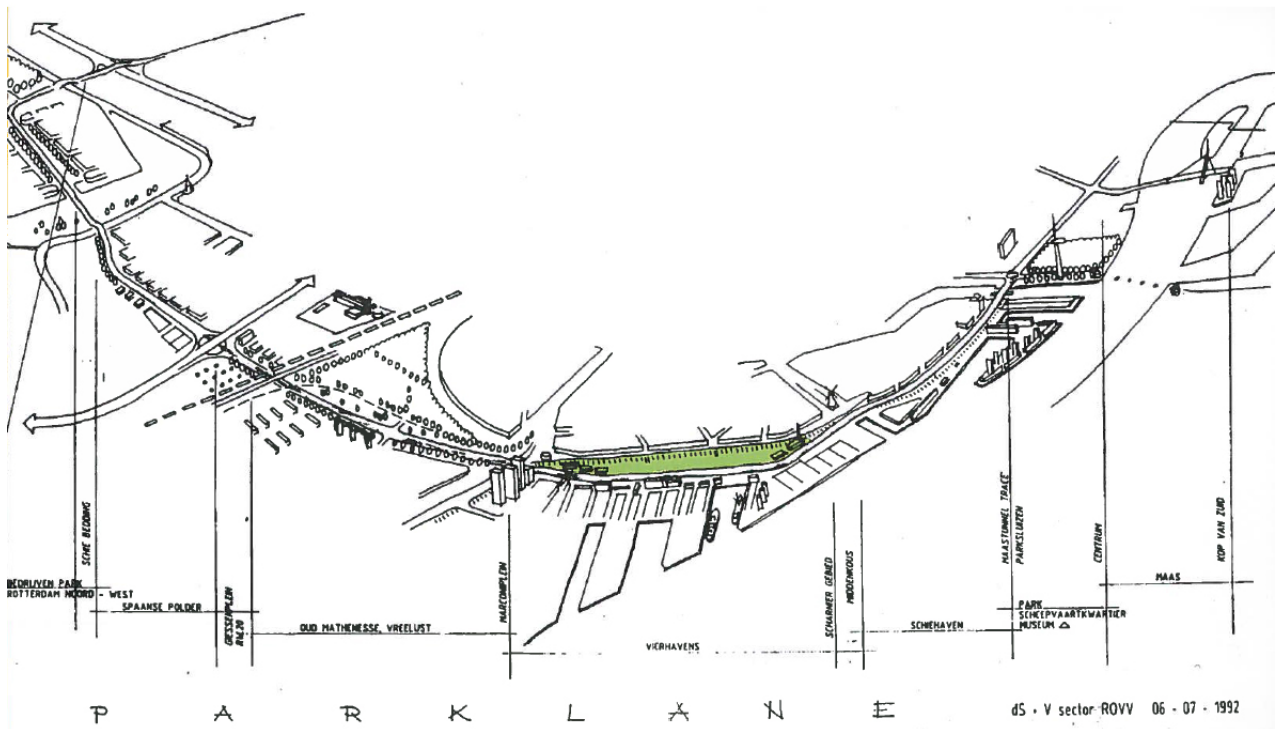

Figure 1.4. Bird's eye drawing of the 'Parklane' concept (Ontwikkelingsbedrijf Rotterdam 2003).

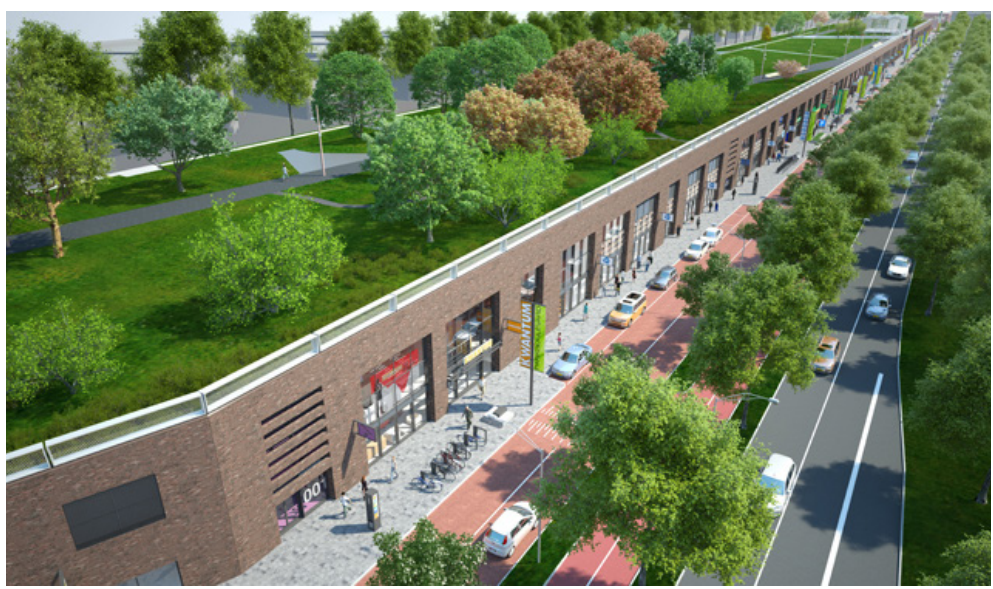

Figure 1.5. 3D rendering of the 'Park Shops Boulevard' (Buro Sant en Co 2007).

processes, each stakeholder - local inhabitants, the municipality, and the project developer, used particular visualization styles and techniques to further their own objectives. Photo collages and scale models were used to gather input from local inhabitants, yet they were also used in a later phase of masterplan development 
to demonstrate the level of participation that took place during the design process. Bird's eye visualizations showed how the park would connect to a more comprehensive municipal strategy, and a 3D artist's impression demonstrated the potential for real estate exploitation.

Although each image was produced for and received by a specific stakeholder group, complete control by producers over who viewed these images was hard. For example, it is difficult to assume that local inhabitants still recognized their 'Top 10' desired functions in the 3D renderings of the project developer. It is also questionable how 'impressed' potential investors would have been by looking at the scale models produced by local inhabitants.

The succession of design representations in relation to the underlying planning and design process shows that certain inspiring visual concepts can become leading in a design process at the cost of the original design assignment. For example, the preservation of the railroad tracks was no longer needed once the stakeholder enforcing the preservation of the train tracks left the decision table halfway through the design process. Consequentially, the necessity of an elevated park evaporated; a simple ground-level park could now suffice. However, the visual representation of an elevated park concept was considered so compelling that the most powerful stakeholders involved, i.e. the municipality and project developer, supported this idea throughout the remainder of the design process. The designs produced to accommodate this support were no longer based on public preferences or on the originally envisioned gentrifying functions. Moreover, the desirability of high-profile commercial competition for local shops in the neighborhood was questioned severely. Rather, the subsequent designs were based more on a visual concept driven by iconic municipal ideals and financial interests.

The planning and design of flood defence landscape areas - such as the Roof Park - involves the knowledge, insights and imaginations of a variety of scientific experts, policy makers and local stakeholders who make competing spatial claims (Jones \& Stenseke 2011). The meaning of visual design representations can change 
over time and differ for each stakeholder group and individual participant. Consequentially, the visual and communicative qualities of visual representations, the setting in which they are produced, and their various interpretations pose important questions about how they help shape participatory planning and design processes.

In this PhD thesis, I study why visual design representations have different meanings for different project stakeholders and I try to determine the communicative qualities of those images that are most important during the participatory planning and design processes of multifunctional landscape projects.

\section{$\triangle$ \\ 1.2 Designing multifunctional landscapes in the face of climate change.}

The Rotterdam Roof Park design process is representative of many current and future planning and design projects as recent developments relating to climate change have led to the increasing engagement of planning and design disciplines with climate adaptation projects (Lenzholzer \& Brown 2013; Watson \& Adams 2010). Traditionally, the construction of flood defence structures was primarily in the hands of water experts and civil engineers. However, the heightened requirements of flood protection areas demand more space than the current dike system; space that is often not readily available (van de Ven et al. 2011). Consequently, the expertise of spatial planners and designers is needed within such projects to address the increasingly important challenge of combining a multiplicity of spatial functions.

In the Netherlands, a vision of robust, long-term flood defence solutions that incorporate existing spatial functions is being put in place through Dutch policy programs such as the Delta Programme (Rijksoverheid 2017; van Buuren et al. 2016; Verduijn et al. 2012). A multifunctional flood defence strategy is required because to adhere to both flood protection norms and spatial requirements the 
Delta Programme looks for solutions such as the so-called multi-functional flood defence (MFFD) concept. Within MFFD landscapes spatial functions like recreation, housing, transport, and ecology are a functional part of the urban or rural environment (Anvarifar et al. 2016; Gralepois et al. 2016; van Veelen et al. 2015). This implies an integration and sometimes replacement of neighbouring spatial functions; a process that can trigger the response of various stakeholders (Matos Castaño et al. 2015).

Intensive stakeholder collaboration during planning and design processes is expected to lead to inclusive co-design activities and thus a viable process outcome, i.e. a protective flood defence system that safeguards and enhances the spatial quality of the landscape and that enjoys broad political and public support (Kothuis \& Kok 2017). The stakeholder configurations associated with MFFD projects are very diverse due to increased multifunctionality; each project needs to engage with different spatial functions. It is not uncommon to find city planners, civil engineers, landscape architects, members from the water board and local inhabitants working together on the same project (Altamrano \& van der Heijden 2013). Moreover, stakeholder configurations differ as projects go through the various analytical, conceptual and implementational design and planning phases (de Jonge 2009; Lawson 2005). These stakeholders have their own backgrounds, experiences, interests and disciplinary traditions and are therefore able to provide insight into planning and design challenges and solutions (van Dijk 2011).

This diversity in stakeholders' perspectives can easily lead to miscommunication when different interpretations of design challenges and solutions clash during a participatory process (Gailing \& Leibenath 2015). The challenge in participatory planning and design processes is to facilitate the communication of design ideas between stakeholders and participants in such a way that the planning and design process will produce more effective collaborative outcomes. To achieve effective design outcomes the expertise of different academic and professional experts 
should be combined, the preferences of local stakeholders taken into account and public and political support for the implementation of the design secured.

\section{$\triangle$ \\ 1.3 Design visualization in landscape architecture and planning}

In general, landscape architects and planners involved in spatial (re)development projects deal with stakeholder groups of different disciplines and various levels of complexity (Southworth et al. 2012; Thering \& Chanse 2011). Visual design representations act as 'communication bridges' that connect the perspectives of landscape planners and designers to those of different stakeholders (Lewis et al. 2012; Schroth et al. 2011; Sheppard et al. 2011; Steinitz 2012). Visual representations are used to gather local knowledge, explain technical details, and inform and persuade specific stakeholder groups using different graphical techniques, e.g. free-hand sketches, photograph montages and video presentations, or digital 2D and 3D perspective views (Barnaud et al. 2013; Lovett et al. 2015; Paar 2006). There are a few comprehensive publications that provide an inventory of the different representation techniques that are used in landscape architecture. These scholarly works range from an overview of historical landscape views and garden designs, axonometric drawings, construction drawings, physical modelling, photography and film, to a handbook for analogue and digital visualization (Amoroso 2012; Balmori 2014; Bishop \& Lange 2005; Mertens et al. 2010; Treib 2008).

These visuals not only facilitate communication, they also serve as a catalyst for discussion (van den Brink et al. 2007) and as a design tool that can be integrated in the design process. (Gänshirt 2007; Pallasmaa 2009). Aside from being an important means of representation, landscape visualizations are also considered as productive entities in the context of planning and design processes. Drawing, for example, is considered as an act whereby a design can be advanced into a further stage of development (Biddulph 2014; Dee 2016). In this sense, representations are 
tools for collecting and synthesizing knowledge and information and, by extension, shaping the process of architectural design (Altürk 2008; Balmori 2014). This means that images not only represent the world, they also make the world (Mitchell 2002) This claim has become increasingly relevant as it is acknowledged that digital visualization techniques are altering the way in which landscapes are designed and are broadening the possibilities for future landscape design interventions (Walliss et al. 2014; Walliss \& Rahmann 2016).

However, Lee and Pae (2017) recognized an inherent paradox in digital landscape design representation. They argue that the most realistically looking images are produced to depict landscapes that are not yet actualized. 'Realism', in this sense, does not refer to the real world but rather to 'an established pictorial convention that traces back to the arts of the early $20^{\text {th }}$ century - and even earlier picturesque depictions of the $18^{\text {th }}$ century - within the discipline of landscape design' (Lee \& Pae 2017: 3). Considering the pivotal role of design representations for the communication and practice of landscape design it is increasingly recognized that the use of such visualizations involves the risk of injecting misinformation into a planning and design process. This issue has become more pressing since modern techniques that are capable of producing hyper-realistic images (Kullmann 2014) are increasingly connected to the 'pervasive culture of design marketing' (Groulx \& Lewis 2017: 10). Linked to the idea of the 'experience economy', i.e. the marketing of experiences as products, it is even claimed that computer-generated images not only market buildings or urban environments but also embody particular sensations (Degen et al. 2015). This means that atmospheres that are artificially produced manipulate the aesthetic perception of the designed object. Moreover, due to their apparent realism, it is often overlooked that 'visualizations are empirical models, calibrated only to characteristics that are selected from the complexity of real landscapes' (Nassauer 2015: 171). Overlooking the selective nature of visualizations is problematic. A recent study has shown that the built reality often differs from the imagined design visualization (Downes \& Lange 
2015), which could mean that hyper-real imagery leads to project expectations that are impossible to meet.

This discussion extends to planning and design imagery that depicts design content on a higher level of abstraction, such as drawings or maps. Sketches, by their abstract nature, represent a concept rather than a physical object (Bafna 2008). For example, it is not uncommon to list emblematic examples of 'architecture', which are part of the architectural canon, that exists only in representational and conceptual form without ever being built e.g. the Cenotaph for Newton by EtienneLouis Boullée, or the work of Giovanni Battista Piranesi (Rattenbury 2002). Yet, the extent to which drawings are able to capture and represent particular aspects of present and future landscapes is an ongoing scholarly debate among landscape architects, since the landscape is 'not only a phenomenon of analysis, but is more significantly something to be made, or designed' (Corner 1992: 144). For Corner, a drawing is never just an object of analysis, but it is also a generative activity. As the creative design process unfolds drawings become concrete and detailed and increasingly approach their represented objects in terms of visual similarity. However, there are limits to the representative powers of drawings. For example, van Dooren addressed the question of whether the aspect of time, central to the ever-changing nature of landscape, can be represented by means of a drawing. He concluded that 'drawings are important in the production of landscape, but they are only one means among others' (van Dooren 2017: 332). The concept of landscape encompasses more aspects than drawings are able to capture on their own, which is why they are always used in tandem with other techniques, such as cartography.

For maps, it is not the promise of realism but rather the condensation of a lot of information into a small format that leads to the observation that 'concepts which might sound unacceptable when expressed in words may become more palatable when represented graphically' (Pojani \& Stead 2016: 383). However, the credibility and authority attributed to cartographic depictions of real-world phenomena often exceeds their inherent flaws and limitations as representational 
entities (Crampton \& Krygier 2006). In planning and human geography, the role of cartography in planning processes, and particularly the political power of maps, is a widely researched topic (Bueno Lacy \& Van Houtum 2015; Crampton 2001; Dühr 2007; Harley 1988). Maps can be both efficient and obfuscating means of communication due to their condensed nature, yet the influence of maps on participatory design processes is rarely addressed in the field of landscape architecture from a critical perspective.

According to Dee (2004), such a critical perspective can inform a critical visual research approach that focuses on looking at visual communication more broadly in terms of convergence between intent and image interpretation rather than looking primarily from the producers' or audiences' viewpoints. A critical visual research approach could be useful because even though design disciplines like landscape architecture rely heavily on their visual language (Gough 2016; Rattenbury 2002), the choice of analogue visual techniques is generally made implicitly and intuitively (Dee 2004). This implicit use of visualizations can be problematic, as the current participatory nature of planning and design processes and the high complexity of the spatial and socio-political contexts of multifunctional planning and design projects call for a more conscious use of these 'traditional' representation techniques.

The apparent centrality of the visual or ocularcentrism (Jay 1993) within spatial design disciplines has led to a fading distinction between 'seeing' and 'knowing'. This subtle merging of concepts influences the way in which knowledge is constructed (Jenks 1995), and by extension to the way architectural works are discussed (Rattenbury 2002) and design ideas are formed (Dee 2004). This means that one of the main challenges for planners and designers is to present design ideas and imaginations in a way that promotes communication and interaction while making clear the limitations that are inherent in each visualization technique (Nassauer 2015). However, developments in the field of digital visualization are mostly technology-driven and are generally evaluated in terms of their technical 
capabilities (Lewis 2012) rather than their use as means of communication. As such, existing critical studies of digital landscape representation most often focus on a single and thus limited perspective. For example, some scholars study visualization effectiveness in terms of interactive knowledge construction in participatory planning processes (Bailey \& Grossardt 2010; Gill et al. 2013; Schroth et al. 2011). Others study usability in terms of user experience of new media and techniques (de Boer et al. 2011; Jallouli \& Moreau 2009; Ruotolo et al. 2013), or affective appraisal (Houtkamp 2012; van Lammeren et al. 2010). Despite their relevance, such perspectives are limited because the meaning of visual materials cannot be derived from just an image itself (Rose 2012). There is always a stage of production, i.e. the circumstances under which an image is produced, and a stage of audiencing, i.e. the (re)negotiation or rejection of meaning (Hall 1997) by specific audiences under specific circumstances.

Visual communication, that is the relationship between images, their creation context, and the way that audiences interpret them, can be conceptualized in terms of a meaning-making process (Jappy 2013; Rose 2012). To enable a conscious use of visualization techniques, a good understanding of the meaning-making processes that shape the interaction between the producers' intentions and the audiences' interpretations of visual design representations is needed (Dee 2004; Lange 2011; Lewis et al. 2012). There have also been some pioneering works in the field of landscape architecture and planning that explored the relation between landscape and meaning, for example, Jørgensen 1998; Lindström et al. 2013; Spirn 1998. However, when it comes to design communication, this understanding of meaning-making is either partial, i.e. focused on only a single stage of visual communication, or too practical and pragmatic, i.e. based on trial and error or disciplinary traditions.

The resulting communication can lead to insufficient integration of scientific and professional expertise with the preferences of local stakeholders and consequently to little political and public support for the outcomes of landscape planning and 
design projects. Considering these limitations, the current use and understanding of visual design representations in participatory processes is insufficient to arrive at design outcomes suitable for the increasingly complex socio-political context and stakeholder configurations relevant to planning and designing multifunctional flood defence landscapes.

Based on the overview presented above this thesis identifies a knowledge gap that is two-fold: epistemologically (1), the disciplines of landscape planning and design lack a critical visual research approach to the study of the meanings of visual representations in participatory planning and design processes; empirically (2), there is a lack of understanding about how these different meanings shape participatory planning and design processes and their outcomes.

\section{$\triangle$}

\subsection{Research objective and research question}

The research objective of this thesis is to contribute to a more conscious use of visual design representations in participatory landscape planning and design projects by studying the visual communication that occurs between designers, experts and stakeholders as meaning-making processes. Identifying the factors of the meaning-making processes that shape the progress and outcomes of participatory planning and design projects could enable more effective and transparent communication in the future.

The knowledge gap and research objective identified in this thesis translate into the following research question:

What meaning-making processes are visual landscape design representations part of, and how do these processes shape the progress and outcomes of participatory landscape planning and design projects? 
In this thesis, I focus on the use of visual design representations as a means of communication within participatory planning and design processes of multifunctional flood defence landscapes. The main subjects of inquiry are therefore (1) visual design representations in their many forms and appearances; (2) the meanings that are attributed to or derived from these representations by designers, planners, and project stakeholders; (3) the participatory planning and design processes of multifunctional flood defence landscape projects where these representations are used. To study these issues, this thesis explores and applies theoretical perspectives and visual research methods and develops a critical visual research approach to studying the meanings of visual landscape design representations in a participatory context.

\subsection{A critical visual research approach}

The process of visual communication that occurs during participatory planning and design processes involves the production and interpretation of visual design representations by different project stakeholders. This process of visual communication can be considered as a process of meaning-making; a producer attributes meaning to a visual design representation, whilst the audience derives his or her meaning from that representation. This process of meaning-making is called semiosis and the study of this process is known as semiotics. As meaningconveying objects, visual design representations and their ability to shape participatory planning and design processes can be conceptualized through a theoretical perspective that is based on the ideas of the semiotician and pragmatist philosopher Charles Sanders Peirce (Peirce 1958, 1992). The Peircean theory of semiotics and the philosophy of pragmatism provide both the theoretical structure for this thesis and the epistemological premises on which the critical visual research approach of this thesis has been developed. 
Pragmatism is a philosophical tradition formed around the writings of John Dewey, William James and Charles Sanders Peirce (Scheffler 2012). From a pragmatist view, 'thought' is not only a way of explaining and describing current reality, it is also a way of problem-solving and anticipating future developments. Furthermore, for pragmatists, the value of any type of knowledge and understanding depends on the practical application of those ideas. The concept of 'design thinking' originates from the same premises as pragmatism (Buchanan 1992). Particularly the idea of abductive reasoning, first concretized by Peirce, resonates strongly within the design disciplines (Deming \& Swaffield 2011; Kolko 2009). Abduction entails a logical inference or hypothesizing of a possible outcome (Peirce 1992). Based on that envisioned outcome, a search for empirical data and guiding principles is undertaken to confirm or continuously adjust the original hypothesis (Dorst 2011). Such an adaptive hypothesis - from a pragmatist standpoint - is not to be confused with the 'traditional' hypothesis that is either verified or falsified (Popper 1972) as it is generally the case in the natural sciences.

The - pragmatic - hypothesis of this thesis consists of the assumption that Peirce's concept of the sign and his theory of 'triadic semiotics' provides a workable conceptual framework for the study of the qualities of visual landscape design representations that shape participatory planning and design processes. Triadic semiotics, in the Peircean sense, constitutes the logic of meaning-making (Peirce 1958), and, by extension, a philosophy of communication (Bergman 2009; Jørgensen 1998). For Peirce, the meaning of the world is mediated by different kinds of signs: sounds, smells, colours, emotions, sentiments, etc. From the pragmatist view on semiotics everything has the potential to be a sign, as long as it means something to someone (Eco 1976; Parret 1984). Peirce distinguishes three sign-components, hence the term triadic semiotics: representamen, object, and interpretant. The representamen consists of the perceivable form of a sign, i.e. its physical manifestation as a sound, smell, form, or image. The object consists of the thing that the sign refers to. For example, a flood map (representamen) represents 
the extent in which an area was or can become flooded (object). The interpretant consists of the interpretation of that object, via its representamen, by someone. The communication of an object is thus mediated by the way in which that object is represented. Together, these three components constitute the concept of the sign (Figure 1.6).

Each sign has a sign-function, i.e. a form of logic that explains how the meaning of that sign is constructed. A sign is therefore not defined as a thing but as a function within the process of meaning-making (Eco 1976). To study a sign one needs to study a sign-function. The sign-function of a visual landscape design representation can be conceptualized as follows (Figure 1.7). Imagine a landscape architect who develops a design concept for a multifunctional flood defence structure. To communicate that design concept (object), the landscape architect

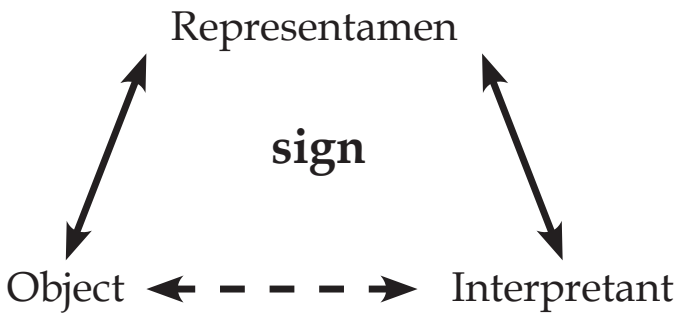

Figure 1.6. Peirce's concept of the sign as a function (Jappy 2013).

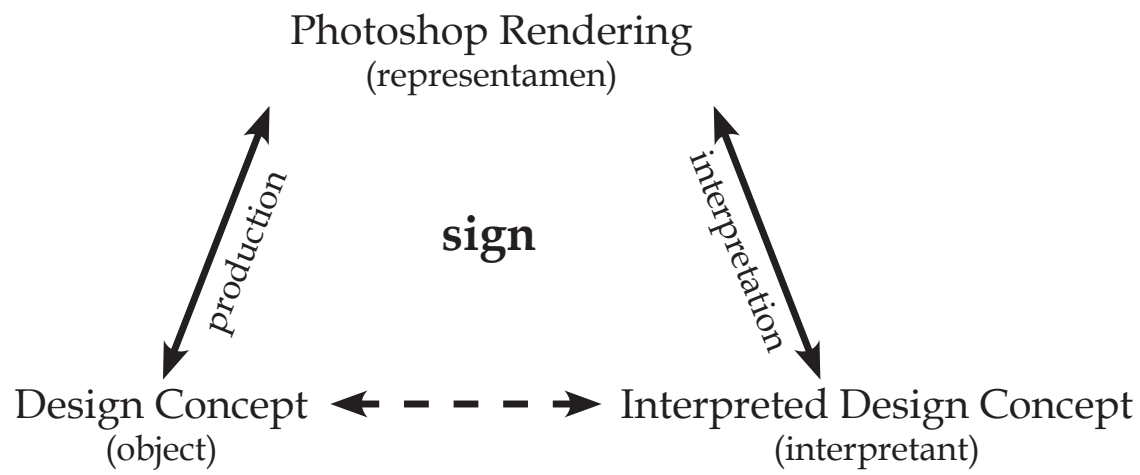

Figure 1.7. Concept of a visual communication process (own figure, adapted from Peirce, Figure 1.6). 
creates a representation of that concept with the use of different visual techniques, styles and media, e.g. a Photoshop rendering presented on a projector screen (representamen). The project's commissioner interprets the image based on his or her professional and personal background and establishes an interpreted version of the design concept in his or her mind (interpretant). This interpreted version of the design concept is a mediated and approximated perception of the original object, which is why this relation is commonly depicted as a dashed line in Peirce's triad (Jappy 2013) (Figures 1.6 and 1.7).

A sign, once it has been interpreted, leads to an action or what Morris called the 'pragmatics of the sign' (Morris 1938), through which it attains a critical function within the design process. In this case, the commissioner might hamper or advance the design process by deciding whether or not to proceed with a further working out of the conceptual design.

\section{0 \\ 1.6 Methodology}

Research approach

The concept of the sign, as described in Figure 1.6, has been operationalized into the main conceptual framework for the research presented in this thesis. This framework is used following the logic of abductive reasoning: the objective or aim is known, i.e. to better understand the meaning-making processes of visual design representations to improve communication. However, the research objects, i.e. the meanings of visual representations and their ability to shape participatory processes are still unknown. At the point of departure the theory and visual methods with which to achieve the objective of this thesis are unclear as well, yet the assumption is made that Peirce's semiotic model can be used as a guiding principle.

This search for theoretical perspectives and methodological applications can be defined as an exercise in 'epistemological pluralism' (Healy 2003). Advocated 
by Feyerabend, the idea of epistemological pluralism means that in the search for scientific 'truth' the researcher uses concepts and methods that 'work' (Feyerabend 1975). The framework developed in this thesis follows this pluralistic principle: the theoretical conceptualization and methodological operationalization presented in each research paper 'work' for the research subject under scrutiny.

Following Peirce's pragmatist conceptualization of the sign, visual design representations can be studied using four distinct foci that line up with the different stages of visual communication: its pragmatics or critical functions, its representamen or image, its interpretation or audiencing, and its object or production. The subject of inquiry of the first focus area consists of the implicit critical functions that landscape design representations have during participatory planning and design processes. Being a theoretical exploration, this analysis entails a critical review of the literature on visual representation, critical social theory, visual research methods, and visualization theories in landscape architecture. The result of this theoretical exploration leads to a proposed critical visual research approach to the study of the meaning-making processes that form a part of visual landscape design representations. Using a case study design, this theoretical research approach is operationalized further for the study of the second (image), third (interpretation), and fourth (production) research foci.

\section{Case study: Rebuild by Design}

The research foci, i.e. image, interpretation and production are studied using an explorative retrospective case study approach. Case study research is useful when studying a phenomenon that cannot be isolated from its original context yet needs to be studied in its totality and in a great amount of detail (Kumar 2014). The selected case should be representative of the subject of inquiry, or be selected based on its a-typical properties. Furthermore, the selected case should be accessible to the researcher so that he or she can learn the most from it (Denzin \& Lincoln 2005). In this thesis, the research phenomenon consists of the meaning-making 
processes of visual design representations that shape the participatory planning and design processes of multifunctional landscape projects that contain complex stakeholder configurations. The empirical case study that is representational of this phenomenon and accessible to the researcher is identified in the form of the Rebuild by Design ( $\mathrm{RbD}$ ) competition. $\mathrm{RbD}$ was a transdisciplinary design competition that took place in the greater metropolitan area of New York after hurricane Sandy in 2012.

The main objective of $\mathrm{RbD}$ was to promote resilience and innovation by developing a multitude of design proposals on a multitude of scales that would prevent future flooding in the area. Key principles for this competition were the ideas that spatial solutions should be multifunctional, connected to local and previous efforts, and strengthen cooperation between governments, businesses, academic institutions, non-profit organizations, and local communities through a participatory planning and design process (Rebuild by Design 2013). Being a design competition there are also additional advantages to the study of $\mathrm{RbD}$. Design competitions are increasingly used to stimulate the quality of urban design and to re-distribute control over that quality amongst participants (Davison et al. 2018). The planning and design processes that take place in the context of design competitions are usually well documented (Chupin 2011), especially if the arrangement of the design process is one of the key criteria for entering the competition. Studying design competition entries also allows for the comparison of designs and design processes, as the project teams are required to work within the same socio-political context and towards similar process outcomes (de Waal et al. 2015).

Within the RbD competition four projects serve as embedded cases (Yin 2009) (Table 1.1). The selected projects are 'Living with the Bay' by the Interboro team, 'New Meadowlands' by the MIT-CAU team, 'Resist, Delay, Store, Discharge' by the OMA team, and the 'Big U' project by the BIG/One Architecture team. The Living with the Bay and New Meadowlands proposals served as the starting point for an 
inventory of visual materials and for the study of the visual complexity of design representations. These two projects were selected on the basis of the comparable scale of their project area (regional), difference in flood risk type (tidal and river, respectively), and accessibility of participants (Dutch design firms and water experts). The Resist, Delay, Store, Discharge and the Big-U projects were added at a later stage and are used to map the broader socio-political context of the $\mathrm{RbD}$ competition for the study of design interpretations and provided additional data for the study of the production stage of the design images.

Table 1.1. Selection of embedded case studies.

\begin{tabular}{ll}
\hline Research focus & RbD Proposal \\
\hline Image (ch3) & Living with the Bay; New Meadowlands. \\
Interpretation (ch4) & Resist, Delay, Store, Discharge; New Meadowlands; Big-U. \\
Production (ch5) & $\begin{array}{l}\text { Resist, Delay, Store, Discharge; New Meadowlands; Living with } \\
\text { the Bay. }\end{array}$ \\
\hline
\end{tabular}

\section{Data collection and analysis}

Case study research is characterized by a 'very flexible and open-ended technique of data collection and analysis' (Grinnell 1981: 302, as cited by Kumar, 2014). Each embedded case study therefore contains elaborate sections describing the reconceptualization of the research subject and a re-operationalization of research methods (Table 1.2). This is done in accordance with the epistemological pluralism described above, which requires the strong contextualized conceptualizations of research subjects to avoid epistemological contamination (Healy 2003).

The study of the first focus area, i.e. the critical functions of design representations, entails a critical review of the literature on visual representation, critical social theory, visual research methods and landscape architecture visualization theories. The image is studied using a visual content analysis and subsequent iconographical interpretation. The study of interpretations and 
Table 1.2. Research methods and data collection methods for all four research foci.

\begin{tabular}{lll}
\hline Research focus & Research method & Method of data collection \\
\hline Critical functions & Literature review & Desk research \\
Image & $\begin{array}{l}\text { Literature review, } \\
\text { visual content analysis, } \\
\text { iconographical } \\
\text { interpretation }\end{array}$ & $\begin{array}{l}\text { Desk research, photo } \\
\text { documentation at design } \\
\text { firms }\end{array}$ \\
& $\begin{array}{l}\text { Literature review, } \\
\text { situational mapping, social } \\
\text { semiotic post-foundational } \\
\text { Interpretation }\end{array}$ & $\begin{array}{l}\text { Desk research, semi- } \\
\text { siscourse analysis }\end{array}$ \\
& $\begin{array}{l}\text { Literature review, visual } \\
\text { discourse analysis }\end{array}$ & $\begin{array}{l}\text { Desk research, semi- } \\
\text { structured interviews }\end{array}$ \\
\hline
\end{tabular}

processes of interpretation is conducted through a social semiotic post-foundational discourse analysis (SSPDA) of semi-structured interviews using the situational mapping technique. Image production is studied using a visual discourse analysis of image materials and semi-structured interviews.

The selected embedded cases were analysed using both quantitative and qualitative data. Image material was collected from the design proposals' documents, brochures, flyers and websites. Also used were analogue sketches that were photographed at design offices, poster boards, scale models and information leaflets that were photographed at a community meeting. This image material was analysed qualitatively, i.e. by manually coding the images, and quantitatively by conducting a co-occurrence analysis, both in data analysis software Atlas.TI. Coding was done according to a rudimentary coding scheme that evolved into a more complex overview of visual and content-related codes after three coding rounds, up until saturation was achieved (Bryman 2012).

Semi-structured interviews were conducted with landscape architects, urban designers, municipality officials, local stakeholders, and scientific experts. These interviews were transcribed literally and coded manually using Atlas. TI. The interviewees were selected based on their involvement with the various 
$\mathrm{RbD}$ projects and their availability for an interview. The interviews with Dutch designers, planners and scientific experts were conducted in 2014 and 2015. The interviews that were conducted with local stakeholders for the analysis of design interpretations took place during a 3-week fieldwork period in New York City and Boston at the beginning of 2016. During this period respondents were selected using a snowballing technique, which led to the inclusion of interviewees from the Resist, Delay, Store, Discharge and Big-U proposals. Consequently, these two projects became additional embedded cases for this thesis research into the interpretation and production of design representations.

\section{Validity}

The internal validity of case study research depends on whether the empirical phenomena can be described in an accurate way (Kumar 2014), i.e. the visual complexity of images, their interpretations and their production. Each empirical research focus involves a qualitative interpretive research method, which ensures that the situations described by specific interviewees are reproduced from their perspective and as accurately as possible (Schwartz-Shea \& Yanow 2012). Such a purely qualitative approach makes it difficult to generalize empirical findings into conclusions about all Rebuild by Design proposals, multifunctional landscape projects or design competitions in general. Rather, the external validity of this thesis depends on the extent to which the empirical case studies enable an application of and critical reflection on the proposed visual research approach. This approach, as defined in the objective of this thesis, should help to understand the visual communication between designers, experts and stakeholders as meaning-making processes. The external validity of each thesis chapter is discussed and evaluated in the relevant chapters and in the final chapter of this thesis. 


\section{$\nabla$ \\ 1.7 Structure of this thesis}

The following four chapters present the theoretical exploration, explication, and application of the critical visual research approach developed in this thesis, each as a peer-reviewed journal article (Table 1.3). Chapter 2 'sets the stage' for a critical visual research approach through the exploration of different theoretical perspectives and visual research methods for studying the critical functions of design representations within participatory planning and design processes. Chapter 3 addresses the semiotic complexity of landscape design representations with a research focus on the image. In chapter 4 , the process of image interpretation is studied. Chapter 5 deals with the discursive power attributed to landscape design representations by their producers. Chapter 6 contains the synthesis of the four research chapters into an analytical framework for a critical visual research approach. This chapter also presents an overview of the different sign-functions and communicative qualities of visual representations that describe how visual design representations shape participatory planning and design processes of multifunctional landscapes. These findings are then discussed in the light of recent scholarly debates to determine their academic contributions to the disciplines of landscape architecture, planning and visual studies. This thesis concludes by reflecting on the research approach, addressing the societal relevance of this research and by making recommendations for further research. 


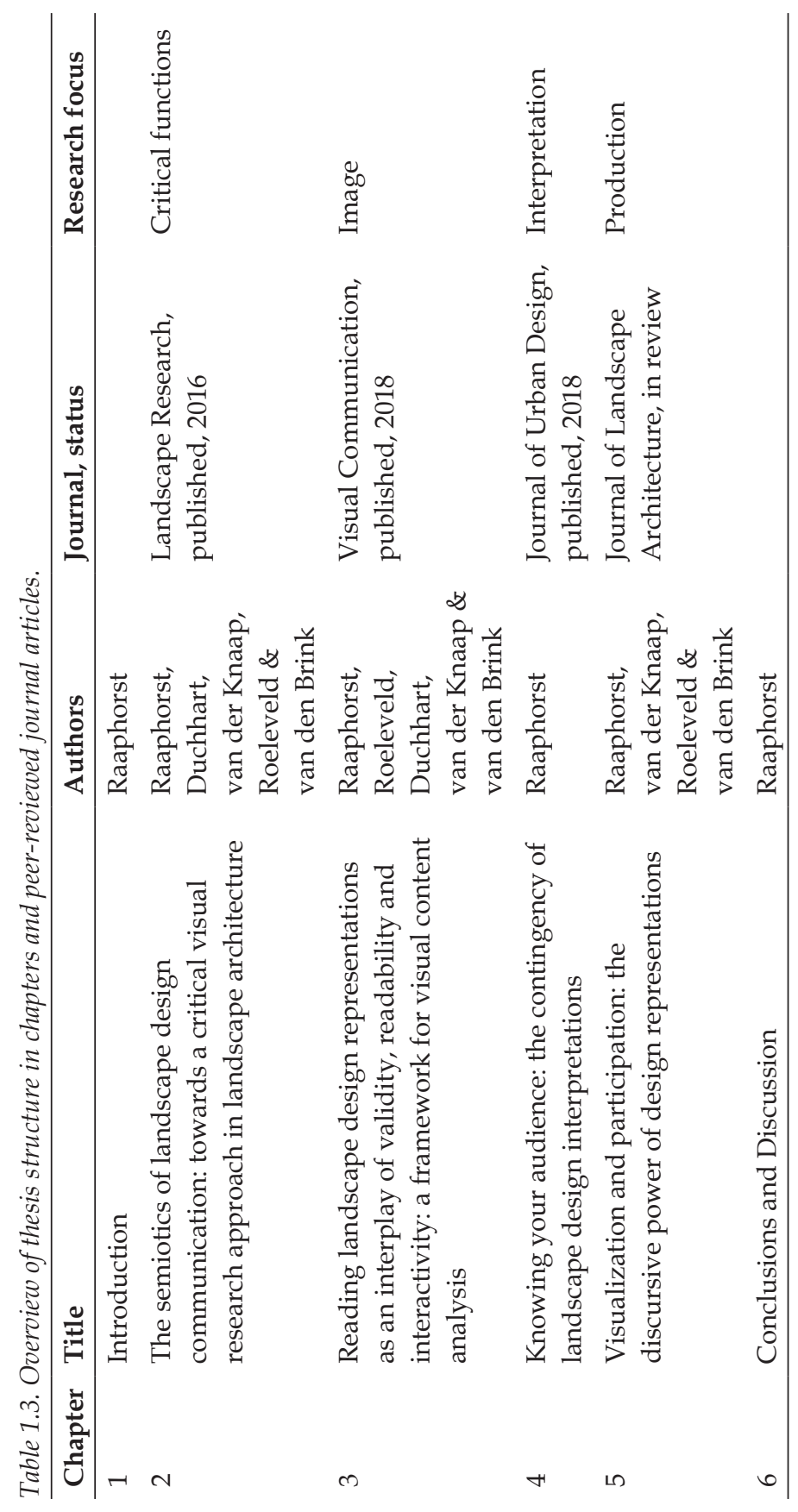




$$
2
$$


This chapter is published as:

Raaphorst, K., Duchhart, I., van der Knaap, W., Roeleveld, G., \& van den Brink, A. (2017). The semiotics of landscape design communication: towards a critical visual research approach in landscape architecture. Landscape Research, 42(1), 120-133.

Keywords

Critical visual research, visual representation, landscape architecture, semiotics, participatory design. 


\title{
Chapter 2
}

\section{THE SEMIOTICS OF LANDSCAPE DESIGN COMMUNICATION:}

towards a critical visual research approach in landscape architecture

\begin{abstract}
$\pi$
Abstract

In landscape architecture, visual representations are the primary means of communication between stakeholders in design processes. Despite the reliance on visual representations, little critical research has been undertaken by landscape architects on how visual communication forms work or their sociopolitical implications. In this theoretical paper, we argue that such research is of great importance. We explain how concepts of visual and critical social theory such as visual semiotics, simulacra and simulation, and power/knowledge can be used to critically reflect on landscape architectural representations. We further propose to study these representations at different stages of meaningmaking by using visual methodologies such as visual discourse analysis, iconographical content analysis, and social semiotic analysis. We conclude that these research approaches have the potential to explain issues such as dominant power structures, miscommunication between participants, and visual pathdependencies during landscape design processes.
\end{abstract}




\section{$x$}

\subsection{Introduction}

Landscape planning and design processes are participatory and transdisciplinary activities, in which experts and laypeople collaborate and play an active role in designing landscape projects (Thering \& Chanse 2011). During these processes visual representations are an important means of communicating analytic findings, abstract ideas, and design principles, and to inform and persuade participants (Mertens et al. 2010; van den Brink \& Bruns 2014). Representations range from free-hand sketches, photomontages and video to digital 2D and 3D perspective views. (Appleton \& Lovett 2005; Barnaud et al. 2013; Paar 2006). As catalysts for discussions (van den Brink et al. 2007) representations help to establish mutual understanding through knowledge construction. The many forms of knowledge, e.g. local, community, scientific, indigenous, applied, and institutional knowledge, are recorded, legitimized, and communicated through visual representations (Cross 2006; Lawson \& Dorst 2013; Lenzholzer et al. 2013). The critical or uncritical use of representations, therefore, determines the ability to communicate different types of knowledge between participants, the quality of a design, and subsequently the quality of the landscape. This issue is not just confined to landscape architecture; professions such as urban planning, interior design and urban architecture deal with comparable complexities.

Many scholars stress doing research into the meaning-making process that influences the interaction between the producers and the users of visual landscape representations, and consequentially the socio-political implications of these images (Dee 2004; Lewis et al. 2012; Perkins \& Barnhart 2005). Recent studies focus primarily on the visualization effectiveness of digital decision-making tools in participatory planning processes (Bailey \& Grossardt 2010; Gill et al. 2013; Schroth et al. 2011), their usability in terms of user experience (de Boer et al. 2011; Ruotolo 
et al. 2013) or the affective appraisal of 3D visualizations and human-computer interaction (van Lammeren et al. 2010).

Dee (2004) suggests further research into critical visual studies that looks at effectiveness and usability in terms of the convergence between image intent and image reception. Besides using the visual as a research method (Dee \& Fine 2005), Dee suggests a research approach that could 'highlight the relationship between drawing form and content, product and process, the subjects they privilege and the damaging effect of the uncritical use of techniques' (Dee 2004, 22). She also suggests addressing the problems and potential of digital imagery, because these techniques can be subjected to the same type of criticism levelled at 'analogue' drawing techniques. Moreover, several scholars observe that a 'technological thrust' has provided unprecedented technological possibilities yet insufficient knowledge on how to use them (Bishop \& Lange 2005; Lewis 2012).

Our aim is to elaborate the critical aspect of Dee's concept by establishing a strong connection between landscape architecture, visual research, and critical social theory. Dee argues that representations can assume a range of critical functions that are often overlooked. We claim these functions connect visual landscape research with a body of work on critical social theory, which is defined as a form of reflective knowledge that aims to reduce entrapment in systems of dominance and dependence (Habermas 1972). By making explicit the implicit social structures that influence human behaviour, we can engage in transformative action that reduces inequalities. The principles of critical social theory are thus similar, if more political, to those of transformative research approaches (Creswell \& Plano Clark 2011) which are already being applied in the field of (landscape) architecture (Groat \& Wang 2013; Lenzholzer et al. 2013). A critical visual approach in landscape architecture aims to make explicit the implicit functions of visual representations during design processes, and in doing so uncover and reduce inequalities between stakeholders.

The research question central to this paper is how can the implicit critical functions of visual representations in participatory landscape planning and design 
processes be made explicit. It therefore focuses on introducing a theoretical and methodological framework for carrying out critical visual research in the field of landscape planning and design. We introduce and combine relevant work on critical social theory and visual communication research available, for instance, in communication science, art history, architecture, media studies, and political geography. First, we discuss and link several theoretical and philosophical concepts that are related to visual semiotics, i.e. visual meaning-making, through a literature review. We then argue how these theories feed into visual methodologies that address the different 'stages' of visual meaning-making: the production, the image and the audiencing stages. We conclude by describing the relevance of a critical approach to design representations with reference to recent developments in architectural education and profession.

\section{$\triangle$}

\subsection{Semiotics}

We start by explaining the relevance of semiotic theory for the study of visual design representations in relation to landscape design processes. Representations are a form of visual communication that involve a collection of visual signs. Every sign engages in a process of meaning-making called semiosis (Eco 1976). Semiotics is considered to be a rich resource for landscape researchers (Lindström et al. 2013) who explore the semiotics of the landscape as an entity. They try to shape and trace meaning within the physical and sensual experience of the landscape (Howett 1987), or to interpret how meaning and identity is reflected in visual design representations (van Assche et al. 2012). We expand on these writings by considering the visual representations of landscape designs as an entity that engages in semiosis.

To understand how semiosis works, and how it might be studied, we present a brief overview of the way semiotic theory developed. Ferdinand de Saussure 
(1857-1913), a historical linguist, presented a 'synchronic' approach to linguistics, which involved a study of the momentary conditions under which language gains meaning in a network of signs; how language functions as it is, not how it came to be (Holdcroft 1991). De Saussure argued that a linguistic sign is a dyad, a twosided entity, consisting of a signifier and a signified (Figure 2.1). A signifier is the physical representation of a sign, such as a written word, an image, or a sound.

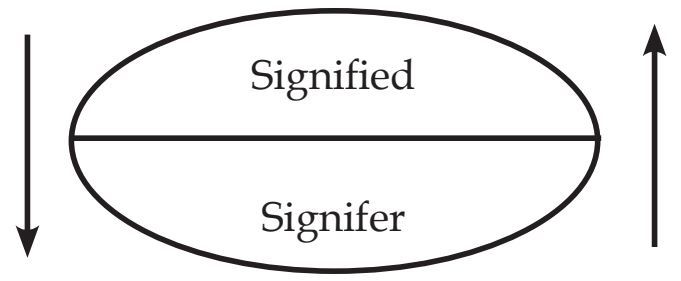

Figure 2.1. Dyadic Model of Semiosis by de Saussure (own figure).

The signified is the mental concept, or meaning, that is attributed to a signifier or a collection of signifiers. In semiotics, communication entails a transfer of mental concepts between individuals through the use of signifiers (Eco 1976). This transfer presupposes a process of constant encoding and decoding, during which meaning is embedded within, and derived from, a signifier.

As a linguist, de Saussure found that the relation between a signifier and a signified was culturally determined. For instance, in landscape design practice, the extensive use of symbols can easily lead to confusion between different participants. The basic treetop symbol, to a layman's eye, resembles a tree as much as it does a parasol (perhaps more the latter) (Figure 2.2). This concept stresses the importance
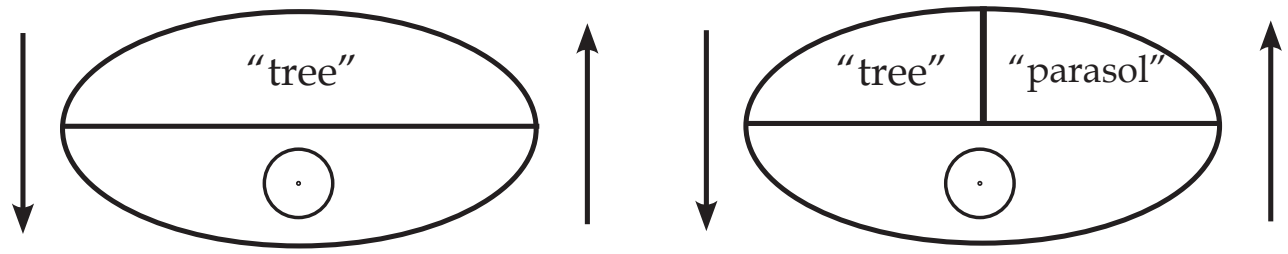

Figure 2.2. Singular (left) and multiple (right) signification (own figure). 
of studying how signs function within society through cultural relations; a study that de Saussure called semiology, a term that is used to describe the European school of semiotics.

In the US, almost simultaneously, the philosopher Charles Sanders Peirce (1839-1914) developed a semiotic theory of logic that advocated a triadic understanding of semiosis. He made a distinction between the representamen (the physical sign), the real-world object that the sign refers to, and the interpretant (a 'proper significate effect' or 'sign within the mind') (Eco 1976) (Figure 2.3). This triadic understanding describes the decoding process that takes place as the audience establishes a new mental sign, or 'interpretant', which can again relate to an object, and thus forms a new 'interpretant'. This potential of 'unlimited semiosis' (Eco 1976, 1990) explains why an image can trigger an endless stream of connotations.

\section{Representamen}

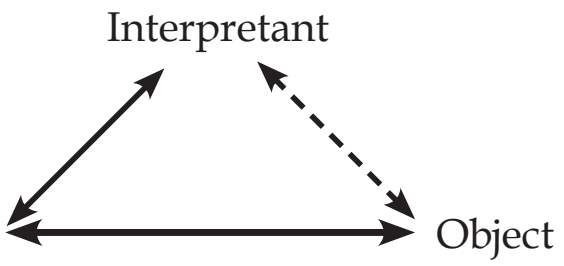

Figure 2.3. Triadic model of semiosis of Charles Sanders Peirce (own figure).

The dyadic theory of de Saussure aimed at culturally determined denotation (what does the sign refer to?), whilst Peircian semiotics opened up the possibility for connotation (invoking other, existing sign systems). According to Peirce's understanding, the treetop symbol would refer to a real-life tree. The mental image of that tree opens up possibilities for connotation: of a tree that provides shade under which to read books, but also of a tree that drops dead leaves that make the ground slippery, people falling and requiring medical attention, etc. These connotations are assembled into a personal interpretation of the meaning of the treetop symbol: an assemblage that is often unpredictable (Figure 2.4). 


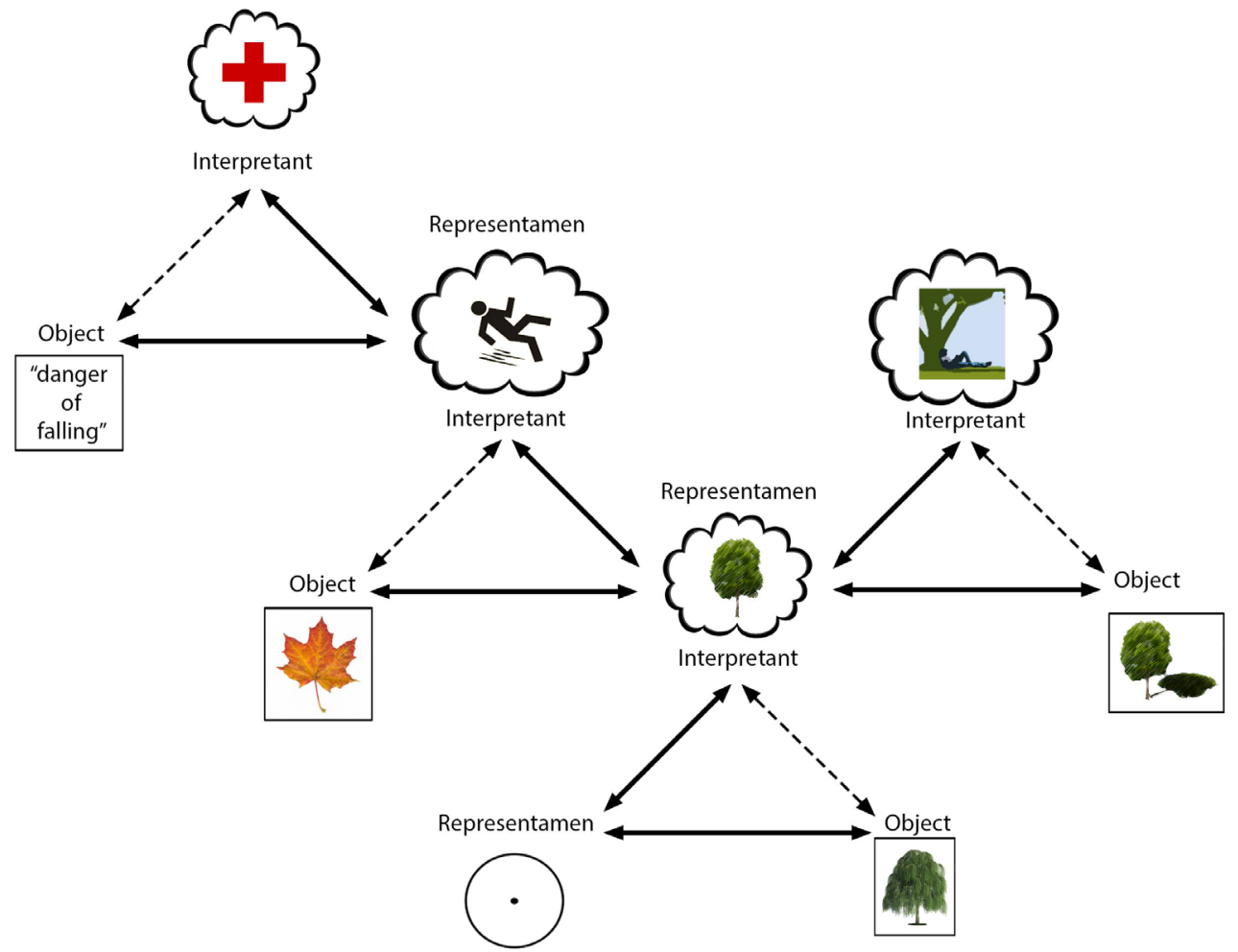

Figure 2.4. Possible assemblage of meaning through a triadic signifying chain of connotation (own figure).

Roland Barthes (1915-1980) is a Saussurian successor who, like Peirce, incorporated the power of connotation. Barthes explained how to deconstruct an advertisement by separating the image into three messages: a linguistic message (the text), a coded iconic message (connotations) and a non-coded iconic message (denotations) (Barthes 1977). In Peirce's understanding, the non-coded message refers to the object of the sign: the apparent denotation. The coded message refers to the interpretant of the sign; the string of invoked connotations. To avoid confusing these concepts, please refer to Table 2.1 for a comparison of semiotic terminology. We have highlighted various aspects of a photomontage and denoted their non-coded message, as an example, in Figure 2.5. We address the coded, 
Table 2.1. Comparison of Semiotic Concepts (own figure).

\begin{tabular}{llll}
\hline de Saussure & Peirce & Barthes & Definition \\
\hline Signified & Object & $\begin{array}{l}\text { Non-coded message } \\
\text { Denotation }\end{array}$ & The referent (i.e. fire) \\
Signifier & Representamen & Sign & $\begin{array}{l}\text { The material sign (i.e. } \\
\text { smoke) } \\
\text { Signified }\end{array}$ \\
& Interpretant & $\begin{array}{l}\text { Coded message } \\
\text { Connotation }\end{array}$ & $\begin{array}{l}\text { The interpretation (i.e. } \\
\text { campfire) }\end{array}$ \\
\hline
\end{tabular}

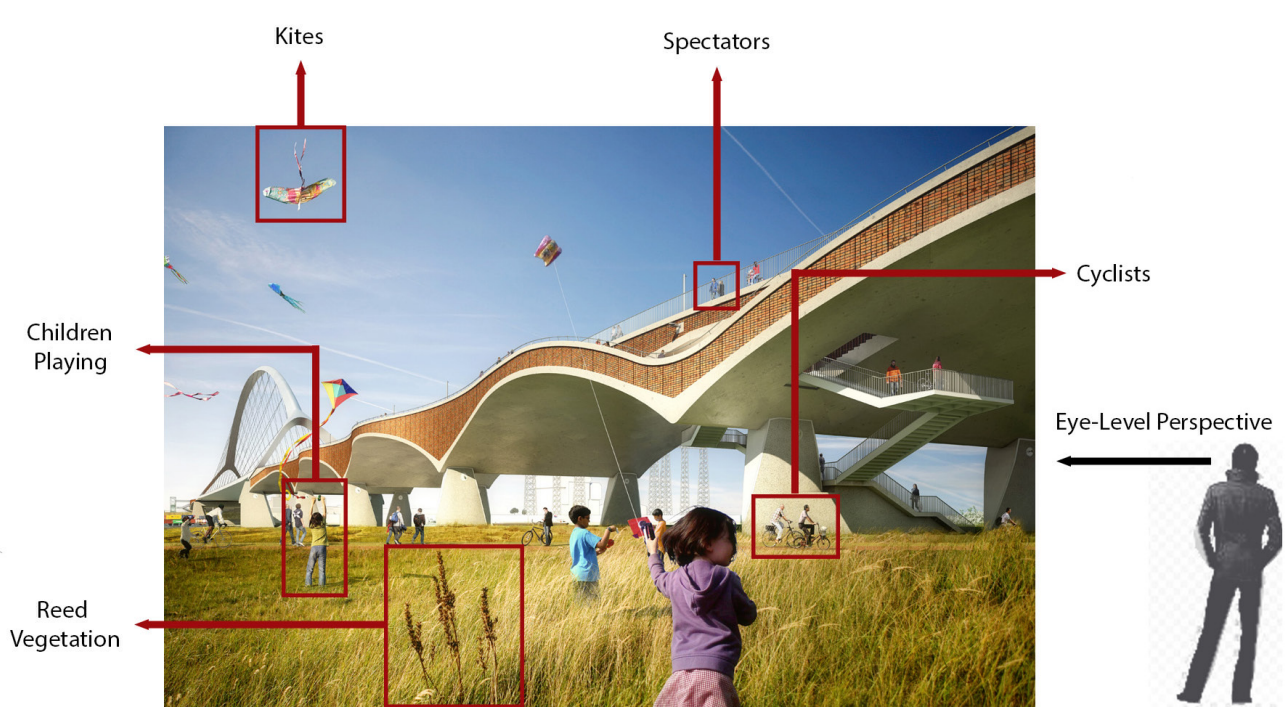

Figure 2.5. Denotative Meaning in a Photomontage (BMD, 2011) (own analysis).

connotative layer of meaning of the same image later in this paper. According to Barthes, the process of connotative decoding is so natural and immediate that it is often difficult to distinguish connotation from denotation. He explains how these natural connotations, or 'grey areas', exist within popular culture as myths and make an unjustified claim to being 'real'. What people perceive as reality is in fact an interpretation shaped by personal experiences, background and beliefs (Barthes 2009).

This perceptual blending of denotation and connotation caused scholars to question whether the existence of a stable 'objective' signified is possible. In fact, 
philosopher Jacques Derrida (1930-2004) claimed that it is not through Saussurian difference (the way a sign differs from other signs) but through Derridean différance (the way the meaning of a sign is deferred, or postponed, until it is influenced by another sign) that the meaning of a sign is continually altered, and thus unstable (Baugh 1997; Gottdiener 1995; Hall 1997). For example, the symbol of the treetop refers to a tree; combined with an empty field it refers to shade; combined with buildings it suggests that this tree could block the view from inside that building. The meaning of one sign is influenced by its relation to another sign. Derrida's différance emphasizes the importance of contextualization, the reconstruction of the sign systems that influence the process of meaning-making (Baugh 1997; Pada 2009).

\section{$\triangle$}

\subsection{Iconography}

This stance on contextualization is similar to that of iconography, an analytic strategy strongly related to Barthesian semiotics. It goes beyond the image alone: it also studies the image's context (Panofsky 1955). Primarily used in disciplines such as art history, iconography - guided by cultural and historical contexts - enables the interpretation of cultural meanings and expressions over several historical periods, including the social class and political regime in which the artist and the audience lived (Rose 2012; van Leeuwen \& Jewitt 2001). Iconographic landscape research has a long tradition within the field of art history and political geography. For instance, a body of work studies the landscape as a cultural image. Landscapes, represented either in a painting, literary prose, or as a cultivated environment, can be recognized as important signs of political, economic and social issues (Cosgrove \& Daniels 1988). Moreover, the designed landscape is also viewed as a cultural and political representation of power that in turn shapes and controls the people who are part of the landscape (Corner 1992; Mitchell 2002). In line with landscape iconography we propose an iconographical study of the power of landscape 
representations. Through intertextuality - the comparison of images from a similar context - we can describe the intentions of the producers of landscape visualizations from a critical semiotic perspective (Rose 2012).

Peircean semiotics, iconography, and Derrida's différance concept can be used to study visual representations of landscape designs. At the macro level, the object of semiosis is the imagined design in the mind of the designer; the representamen is its visual representation, and the interpretant is the way in which different participants in design processes interpret the design. At the micro level, the scale, the perspective, the use of certain colours, building materials, and figures all refer to certain coded (interpretant) and non-coded (object) messages. These layers of meaning illustrate the complexity of effective design communication. Moreover, it shows that not every visual representation refers to reality or to the imagined reality in the same way.

\section{$\triangle$}

\subsection{Simulacra and Simulation}

Derrida's unstable signifieds make us question the extent to which a landscape design representation can legitimately represent an imagined reality or landscape. These socio-political implications of semiosis were explored by Jean Baudrillard (1929-2007) in a post-structural turn in semiotic theory. Baudrillard claimed that it is not reality that emits signs in order to be perceived, but that the signs themselves construct reality as simulations. Signs circulate increasingly up to the point that their domination becomes so complete that they replace reality entirely and the representation becomes more important than the reality it aims to represent. This precession of simulacra, as Baudrillard called it, posits the idea that the only way we can perceive reality is through the mediation of reality by culture and media (Baudrillard 1981, 1988). 
Baudrillard's critique concerning the dominance of signifying practices has inspired critical theory about the relationship between (urban) architecture, culture, and representations (Proto 2003; Rattenbury 2002). These theorists argue that, despite the fact that architecture is driven by physical reality, designs are generally discussed through visual representations. Even unbuilt, imagined designs sometimes claim the same architectural status as their built counterparts blurring the distinction between the real and the unreal. This 'hyper-real', so they argue, might very well lie at the core of our definition of architecture.

Simulacra and the concept of hyper-reality have gained increasing attention with the rise of modern 3D modelling techniques that give unprecedented levels of visual detail and a sense of realism (Kullmann 2014). However, the sociopolitical status of imagery is equally important. A concrete example of this is the political power of cartographic images. Maps have credibility and authority. They are assumed to represent the world 'as it is' (Bushell 2012; Crampton \& Krygier 2006). Maps can also be used to distort reality (Monmonier 1995) because they mediate reality for us, not through a comprehensive gaze but through a regime of mathematical equations, political choices and financial interests (van Houtum 2013). The cartographic use of scale, level of detail, and perspective reduces the complexity of reality to simplified symbolic representations (Wohl \& Strauss 1958). For the majority of the population the only idea of place that exists outside the reality of their own personal experience is mediated through this subjective cartography and representation (Menatti 2011).

The precession of cartographic simulacra occurs when representations of reality shape reality itself. For instance, the incorrect depiction of state borders between Costa Rica and Nicaragua by Google Maps flared up a long-standing border dispute and lead to military action (Brown 2010). This suggests that if it is on the map it is true, for it is part of the cartographical simulacrum. This dominant view ignores the unmapped places that often speak more to our imaginations than their charted rivals (Bonnett 2014). Similarly, the credibility of design representations 
shapes the expectations of the built reality. This issue is especially relevant if we consider the temporal nature of landscapes: a design might take 20 years to come to fruition.

Different stages of the simulacrum depict the degree of abstraction from reality - i.e. the conceptual distance between the interpretant and the object - and thus the similarity between the envisioned and the built landscape. One could argue that a landscape design can potentially become a simulacrum when certain semiotic strategies (colour scheme, perspective, figurines, weather effects) create strong expectations in the early stages of the design process. This can be intentional or unintentional, and depend on the visual (il)literacy of the audience or on different 'visual languages'. If this image looks too real or definitive and becomes a benchmark for the designs that follow, successive visualizations could enforce the initial misrepresentation by making it more specific, detailed and 'real'. At its final stage the design no longer holds any reference to the built reality. In more practical terms, this precession of simulacra is captured by the phrase 'it looked better in the pictures'.

\section{$\triangle$ \\ 2.5 'Power/Knowledge'}

Considering the influence of visual representations on the perception, understanding, and evaluation of the (imagined) landscape, the production of knowledge, and thus the design process, criticism should focus on the social relations within which the production of visual representations is embedded. Regard for the different social positions of the producers and the users of visual representations is lacking, despite the fact that the power relations between these two groups define what can be represented. Few people and institutions control the content and availability of visualization techniques such as GIS or AutoCAD, and not all participants possess the expertise to apply these techniques. Because of this inequality in the ability to visualize, many theorists acknowledge it to be a 
'critical imperative to examine in detail how certain institutions mobilise specific forms of visuality to see, and to order, the world' (Rose 2012: 10).

In regard to issues of ability, power and control, we follow the argument that Western thought is dominated by ocularcentrism (Jay 1993): one can make a claim to truth through visual representation. Therefore, we regard the act of creating visual representations of landscape designs as an act of knowledge construction; as making a claim to truth. In landscape design practice, participants can make these knowledge claims by sharing and applying their knowledge, expertise and ideas through design representations, either by drawing themselves or through the mediation of landscape architects. The (in)ability of participants to communicate their knowledge determines the credibility of that knowledge. We propose to study this issue through theory that relates to the intersection of power and knowledge.

According to Foucault, power and knowledge are inextricably linked (Foucault 1980). Although he never wrote specifically about landscape representation, his view on power is nevertheless relevant. Foucault's project was not about defining an absolute truth (he considers 'truth' to be highly subjective), but, among other things, about describing the production of a multiplicity of truths by analysing how power functions within society. Power is everywhere and can consist of many things: political influence, skill, expertise, experience, charisma, literacy, and ultimately: knowledge. Power is used to make a claim to truth through knowledge construction. In doing so, power shapes specific knowledge about the world and the way people act on that knowledge. This understanding of knowledge is called discourse. According to Foucault, discourse causes people to think and act in certain ways not because it functions through oppression or the imposition of rules, but because discourse also produces the subjects it governs. Our ideas about places, landscapes and objects consists of those ideas that are presented to us as the truth. Discourses are produced based on the claim that their knowledge is 'true'. The discourse that substantiates this claim most effectively is the one that becomes dominant (Foucault 1974). Therefore, in this paper, we consider visual 
representations of landscape designs not only as objects of semiosis, but also as a discourse: specialized forms of knowledge that are constructed by specific actors and influence the way people act upon that knowledge (Rose 2012).

Stakeholders that present their ideas, knowledge, and design solutions in the most comprehensible and persuasive way are the ones that become dominant during a design process. If we relate this to semiotics, a power dynamic at the production side of design representations a priori influence the interpretations of those designs. This power dynamic of struggling discourses is therefore not only a dynamic of agency (the decisions of the image producer), but also a dynamic of materialisation (what the visual representations of landscape designs, and thus power, look like).

\subsection{Visual Methodologies}

The three main theoretical concepts that we have presented, namely semiotics, simulacra and simulation, and power/knowledge, tie into several visual methodologies that can be applied to study visual representations of landscape designs. Closely related to Peirce's triadic understanding of semiosis, Rose (2012) distinguishes three meaning-making 'sites': the production, image, and audiencing site. These three constitute, respectively, how an image is made, what it looks like and how it is interpreted by the user. To avoid confusing these 'image sites' with physical landscape sites, we choose to refer to these sites as 'stages' of meaning-making.

Each stage constitutes a different focus of visual research. At the production stage, the focus is the social and institutional context within which images are produced. At the image stage, the focus lies on the image itself, what it looks like, its medium and the visualization techniques that were used. At the audiencing stage, specific attention is paid to the way the audience interprets the image, but it is sometimes also necessary to trace how an image functions within a larger socio- 
political context. These are all different aspects of the meaning-making process, and can be studied using different visual methodologies.

The choice of visual methodology and analytical depth depends heavily on the context, the availability of visual data, the interpretive skills of the researcher, and the willingness of participants (Rose 2012). Given the complexity and diversity of design processes, there is no 'one way' to do critical visual research. Nevertheless, numerous methods can be drawn from related scientific disciplines and used to conduct such a study. In her book on visual methodologies, Rose (2012) discusses methods such as compositional interpretation, content analysis, semiology, psychoanalysis, discourse analysis, and ethnographic methods. Van Leeuwen and Jewitt (2001) suggest methods such as visual anthropology, social semiotics, and ethnomethodological approaches. Others advocate visual ethnography (Pink 2012) and critical semiotic approaches (Spencer 2011). Using different but complementary methods to study several image stages generates a deeper understanding of semiosis, but for each study a clear prioritization is necessary to avoid methodological excess and disjointedness.

We will explain how three interpretive methods can be combined within a critical visual research approach for landscape design processes: iconographical content analysis, visual discourse analysis, and social semiotic analysis. These methods focus on different stages of meaning-making, yet overlap: production, image and audiencing are always related.

\section{Image Stage: Iconographical Content Analysis}

We consider collecting visual data as the starting point of a comprehensive visual analysis and begin by addressing the visual methodologies related to the image stage. It is clear that it is not just how the image looks, but also the sociopolitical context that determines how a design is interpreted. We should, therefore, relate the specifics of an image to its maker as well as to its audience. To do so, we can use an iconographical content analysis. This method allows us to make an 
inventory of the visualization techniques that are used in a specific design process. Similar to a traditional content analysis, source material is analysed using a coding scheme which allows the quantification of data characteristics. In the context of landscape design, one can look at the kinds of visualization techniques, media, drawing perspectives and colour schemes.

The coding scheme can also be more detailed and in-depth if we relate this to semiotic theory. A content analysis enables us to study semiotic strategies within specific design contexts. For instance, which shade of green is used to depict trees within the design? A darker shade of green can promote a sense of consistency, history and preservation, while a brighter shade of green implies spring, new beginnings, a futuristic design and human progress. A bird's eye perspective signifies control over the landscape and the ability to shape it to your liking. However, an eye-level perspective appeals to the human sense of scale and to sensory perception (van Leeuwen \& Jewitt 2001). Modern photomontages tend to include human figures on streets, balconies and benches. These figurines attribute meaning and function to certain spaces. For instance, children running through a field of high grass while flying a kite implies a safe environment for children (Figure 2.6). This could very well be a distortion of reality, a simulacrum: the actual field in which the girl is playing could be located next to a busy traffic intersection of busy roads.

Semiotic strategies do not stand alone. They have been implemented by someone at a specific moment in the design process and for a specific reason. Based on an iconographical overview of semiotic strategies, the researcher can identify particular points of interests and decide on the direction in-depth follow up research should take. For instance, the next step might be to conduct a visual discourse analysis in order to study the production of these images or to engage in a study of social semiotics to address the audiencing stage. 


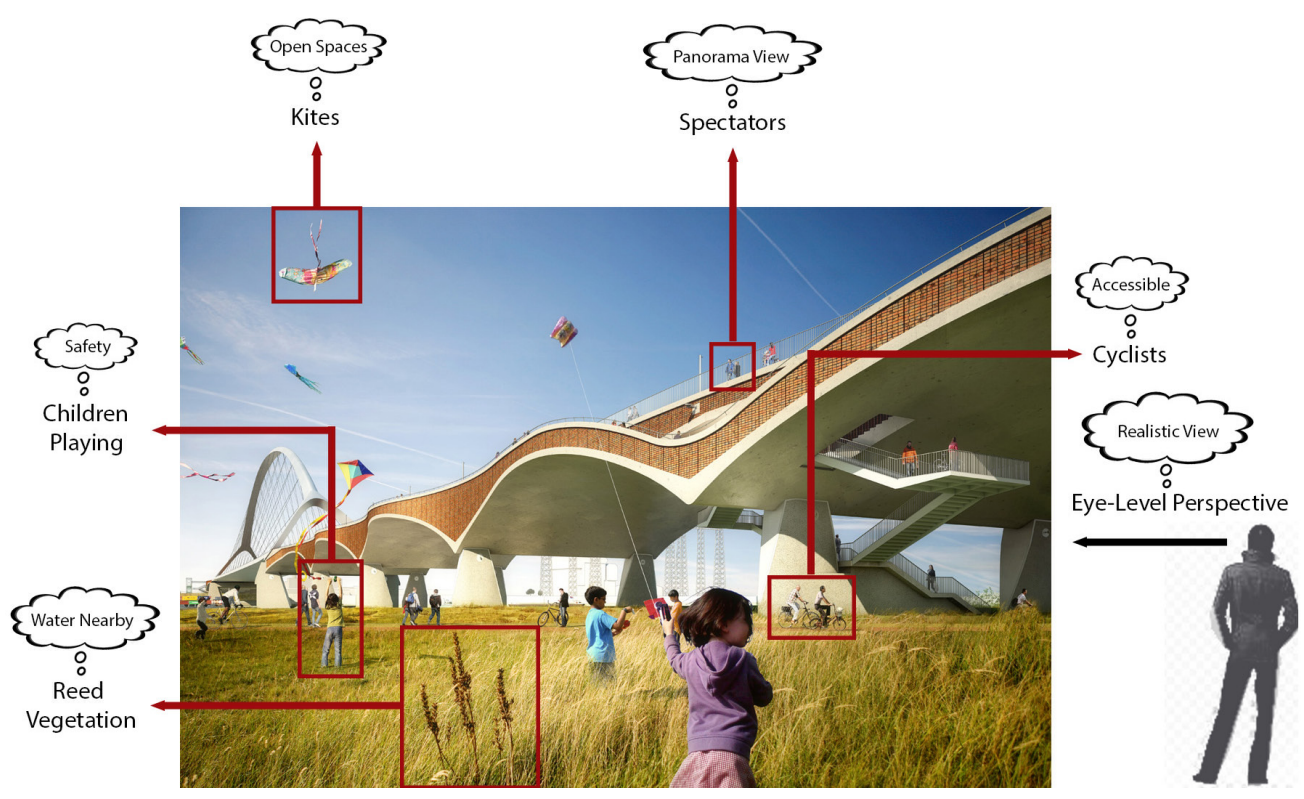

Figure 2.6. Denotative Meaning and Possible Connotations in Photomontage (BMD, 2011) (own analysis).

\section{Production Stage: Visual Discourse Analysis}

For the production stage, we refer to the concept of power/knowledge and define visual representations of landscape designs as discourses. If we consider visual discourses as the materialization of power, then it is imperative to question whose power is being represented, and what this power consists of. To study how a particular discourse is structured and how it produces certain kinds of knowledge, Rose (2012) suggests a Foucaultian visual discourse analysis. Foucault argues that the institutional location of the discourse should be studied by analysing statements in terms of the position of the speaker and his or her social status. To do so, one should look at how the institutions concerned function by focusing on what Foucault calls their apparatuses and technologies (Foucault 1974). The apparatus of the image producer can be defined as - and this is where we observe an overlap with the audiencing stage - the environment where the image is presented: for example, an exhibit at a convention centre, a presentation at the offices of municipal 
planners, a workshop at a community centre, a poster at a bus stop, or a newsletter viewed in the privacy of your home. Likewise, we adopt a broad definition of the technologies a producer may use, for example, the panels on which a design poster is printed, the 3D model on virtual reality glasses, the charcoal of an artist's sketch, and even the watermarked stationery on which a report is printed. Once again we note an overlap. The image stage is intertwined with the production stage.

Foucault presented several distinct methodologies in the course of his career, e.g. discourse studies, genealogy, but he never prescribed any concrete analysis techniques. Rather, his works provide a framework with 'things to look for' when applying techniques of data collection (Kendall \& Wickham 1999). Some of the main questions within a visual discourse analysis relate to the social context: who produces the image; what is the producer's social authority; what producerspecific medium or materials are used; what is the preferred meaning (Hall 1997) of the image; and in what kind of physical environment was the image presented to its audience? Techniques such as in-depth semi-structured interviews with the producer of a representation can be applied for a reflective case study, as well as interviews with the intended audience, to elicit elaborate descriptions of the communication process. Furthermore, observation techniques can be applied reflectively, or during a more experimental set up in an on-going design process, to ascertain the characteristics of the physical apparatuses involved.

\section{Audiencing Stage: Social semiotics}

The extent to which the intended meaning, established through a visual discourse analysis, was conveyed to its intended audience can be studied at the audiencing stage through social semiotic analysis. Social semiotics is the study of the social effects of implicit semiotic strategies on the perception of human artefacts (Hodge \& Kress 1988; van Leeuwen 2005). Derrida's concept of différance adds the notion that the meaning of signs is constantly influenced by the ever-changing context of a sign. If we consider the landscape planning and design process 
as a sign-system, the meaning-making of an image does not occur at a specific moment in time, but rather during a process of agency in time. Influenced by the changing context the interpretation of these images will also change. As a result, images acquire a variety of socio-political statuses within different social groups at different stages of the design process.

This diversity of meaning and social status connects the audiencing stage to Baudrillard's theory of the simulacrum. The research objective is to trace the precession of landscape design simulacra through contextualization: by reconstructing the social, political and institutional contexts in which the image was received. We focus not only on the production of discourse (the intended meaning) but primarily on the reception of discourse (interpretation). Social semiotics, therefore, complement visual discourse analysis by describing the interpretation of the different precession stages of a landscape design simulacrum. In this way we can trace the simulacrum at the production stage and describe the socio-political functions of the images at the audience stage. In terms of knowledge construction: a simulacrum occurs when the interpreted knowledge differs greatly from the represented knowledge.

To do so, we need to determine the lifespan of these images and establish a timeline to describe the 'functional' moments in the design process. This enables us to trace whether, for different stakeholders, the socio-political function of a design visualization has changed over time. This can also be related to the concept of power/knowledge: if an image indeed assumes an intended socio-political function, to what extent was this determined by the apparatus and technologies used by the producer?

To answer these questions, semi-structured interviews can be used. To attain so-called 'socio-semiotic responses', including, for example, detailed descriptions of how respondents experienced the use of design representations, a 'probing' strategy can be used. These probes can be verbal, i.e. follow-up questions, nonverbal i.e. body language (Bernard 2011) or material using stimulus objects 
(Törrönen 2002), such as design representations. The interview strategy can consist in the first instance of broad, general questions that set the stage for a wider topic, e.g. the design process, communication techniques, or knowledge production. Based on these general descriptions, probes are used to elicit responses to specific topics, e.g. participatory design workshops, sketches, 3D visualizations and the mutual understanding of situated knowledge.

\section{$\nabla$ \\ 2.7 Conclusions}

In this paper, we argue that by connecting visual and critical social theories and methods of other visually oriented disciplines, e.g. architecture, art history, and human geography with those of landscape planning and design, a comprehensive critical visual research approach can be developed. We have indicated how theoretical concepts such as semiotics, iconography, simulacrum and simulation, and power/knowledge, provide insight into the meaning-making process that design representations engage in and how these images attain a certain sociopolitical status during design processes. Also why they should be considered to be - and treated as - an important tool in the construction of knowledge.

Semiotics and iconography teach us that there are as many meanings as there are stakeholders. Peirce's triadic system of object-representamen-interpretant explains the logic behind connotation, and illustrates the complexity of meaning. It is essential to consider this complexity in order to develop a mutual understanding of design challenges and solutions. The theory of the simulacrum provides an insight into hyper-reality; how representations relate to the (imagined) designed reality and how an uncritical use of visual techniques can influence future design decisions and create expectations that might be impossible to meet. The concept of power/knowledge explains why the ability to visualize design ideas is an 
extremely powerful ability, and that a more equal distribution or mediation of this ability amongst stakeholders is desirable for a fair design process.

The visual methodologies presented in this paper enable studies of various analytical depth. Iconographical content analysis can prove to be a very effective tool for establishing an initial overview of the characteristics of visual material. Subsequently, the researcher might decide to engage in a more detailed iconographical study of visual semiotics or focus on an in-depth visual discourse analysis. In a closed design environment, the audience of an image might be easily traced and this will enable a detailed social semiotic analysis of personal meaning-making. In a more public design process, the researcher might prefer a more general social semiotic analysis in order to determine the social effects of a landscape design over time.

Considering the social complexity of design processes, we do not claim to offer a 'recipe' for carrying out critical visual studies, nor do we advocate a standardized visualization approach to design processes. Such strategies would impair the creative process as well as ignore case-specific circumstances. Rather we plead for a critical visual research approach tailored to the specific type of design process, the stakeholders that are involved, and the visual materials that are available. We argue that the theories and methods presented in this paper are particularly relevant, yet we also encourage other approaches that contribute to a comprehensive critical visual research approach.

Our proposed research approach can help make the process of meaningmaking between producers, images and users more transparent. Furthermore, it emphasizes the need to think critically about the recent trend of outsourcing visualization activities to third-party graphic designers to meet the market's graphic expectations. These practices externalize the design phase during which most expectations are raised. We therefore conclude by saying that a reflective, knowledge based and transparent use of design representations, in both practice 
and education, could contribute to a wider acceptance of visual studies as a communicative and transformative research strategy.

\section{只 \\ Acknowledgements}

The research for this article was carried out in the context of the research programme 'Multifunctional Flood Defences', funded by the Stichting Technologie \& Wetenschap (STW), part of the Netherlands Organisation for Scientific Research (NWO). Additional 'in-kind' funding has been provided by knowledge institute Deltares. The authors would like to thank the Editor and the anonymous reviewers for their kind words and constructive criticism on the earlier version of this paper.

Notes

The photomontage used for Figure 2.5 and Figure 2.6 is entitled: Stadsbrug 'De Oversteek' in Nijmegen, Design: Ney Poulissen, Visualization: BMD. 


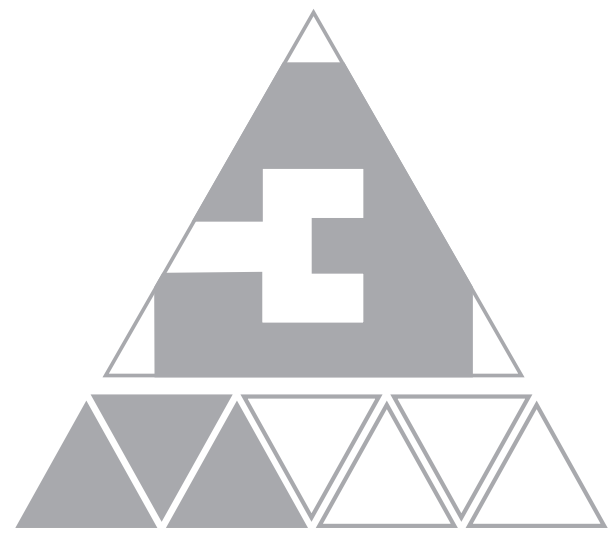


This chapter is published as:

Raaphorst, K., Roeleveld, G., Duchhart, I., van der Knaap, W. and van den Brink, A. (2018). Reading Landscape Design Representations as an Interplay of Validity, Readability, and Interactivity: A Framework for Visual Content Analysis. Visual Communication. (Online first).

\section{Keywords}

Visual content analysis, semiotics, iconography, landscape architecture, design representation. 


\title{
Chapter 3
}

\section{READING LANDSCAPE DESIGN REPRESENTATIONS AS AN INTERPLAY OF VALIDITY, READABILITY AND INTERACTIVITY:}

a framework for visual content analysis

\begin{abstract}
$\pi$
Abstract

Considering the importance of visual representations for communication between stakeholders in landscape planning and design processes, we identify a lack of critical visual research methods supportive of the disciplines involved. As part of such a method we have developed an analytical framework based on semiotic and iconographic theory that enables a visual content analysis and iconographic interpretation of landscape design representations. Two projects from Rebuild by Design, a participatory transdisciplinary design competition organized in the New York City area after hurricane Sandy, were analysed to demonstrate this framework. Our paper presents a semiotic vocabulary based on four categories: medium, mode, formulation and knowledge with which to 'read', discuss and potentially create design representations. This enables a syntactic analysis for assessing the semiotic complexity of design representations in terms of validity, readability and interactivity. This assessment enables further qualitative study of the production and interpretation of landscape design representations in practice.
\end{abstract}




\section{$x$}

\subsection{Introduction}

Landscape planners and designers find solutions to a broad range of spatial issues on a wide range of scales. Current issues include human health and wellbeing with attention to climate, water and energy as well as spatial quality issues arising from ongoing urbanisation (van den Brink \& Bruns 2014). In addressing these issues, landscape planners and designers use diverse visual representations such as sketches, drawings, maps, models and video, to express design proposals for future spatial lay-out and aesthetic quality to an increasingly diverse set of stakeholders (Raaphorst et al. 2017).

The legitimacy of these design representations has become crucial due to the decisive role they play in the range of political, social and ecological challenges that landscape planners and designers face. Moreover, whereas previously landscape plans and designs might have been considered as blueprints for construction, nowadays they are tools (Mertens et al. 2010) for finding possible multifunctional and integrated landscape solutions (Thering \& Chanse 2011; van den Brink \& Bruns 2014). The objectives of these representations vary during participatory planning and design processes. Sometimes the visuals have to provoke an active debate, or maybe they have to illustrate jointly made decisions. Moreover, design images have to engender effective communication between people with different levels of visual literacy. Therefore, effective visual communication in participatory processes with an increasingly diverse group of stakeholders, like decisionmakers, NGO's, community-based groups and the general public has become a vital component of landscape planning and design processes.

Although a number of studies relating to visualizing landscape transformations exist, most of them discuss, for example, different materials (e.g. pencil, paint, paper, models) (Pallasmaa 2009), skills (e.g. drawing in perspective, the use of colour) (Treib 2008), sensorial qualities (e.g. hyper-realism, atmosphere 
drawing, brightness and contrast) (Amoroso 2012; Kullmann 2014; Lewis 2012) and techniques (e.g. photography, digital modelling, GIS, CAD) (Macdonald 2012; Mertens et al. 2010). Other literature explores the potential use of digital technologies in the act of designing (Nijhuis 2015; Steinitz 2012; Walliss \& Rahmann 2016). However, the study of design representations as communicative entities, i.e. both the visual qualities and the content of the image and its relationship to the planning and design process and its phases, is scarcely addressed or only in general terms.

There is a lack of a critical visual research approach to studying visual landscape design representations in a comprehensive way (Dee 2004). In developing such a critical visual research approach (Raaphorst et al. 2017) we aim to contribute to an understanding of the principles of effective visual communication in participatory landscape planning and design processes. Although research on visual methodologies generally identifies three stages in visual meaning-making: the production, the image and the audiencing stage (Rose 2012), in this paper we will focus primarily on the analysis of the image stage. We argue that, before studying the production and audiencing of images, there is a need for a good understanding of the visual content of landscape design representations (Raaphorst et al. 2017).

We draw on semiotic and iconographic theory to address the multiplicity of meanings that can be attributed to landscape designs and their representations. We argue that studying this multiplicity of meaning in terms of semiotic complexity helps to explain and anticipate the effectiveness of design communication between stakeholder groups and experts of different disciplines. In this explorative study, we address the question of how the semiotic complexity of visual landscape design representations can be analysed systematically. In answering this question, we developed an analytical framework for a visual content analysis and iconographical interpretation of landscape design representations.

We demonstrate this framework by applying it to designs used in two participatory design projects. These projects were part of Rebuild by Design (RbD), 
a design competition for generating multifunctional landscape solutions using an explicitly participatory and collaborative approach, set in the greater metropolitan area of New York in the aftermath of hurricane Sandy. The objective of this demonstration is to define a semiotic vocabulary with which to study the semiotic complexity of landscape design representations in a participatory setting.

This paper is structured as follows. First, we describe how we can look at landscape, its representations and landscape planning and design processes from the point of view of communication. In the second section we explore how this landscape perspective to communication can be applied in a visual content analysis of the $\mathrm{RbD}$ competition submissions. Finally, and in conclusion, we show how the images can be described in terms of their visual content and semiotic complexity and we assess the potential communicative qualities of the images through an iconographical interpretation.

\section{$\triangle$}

\subsection{Landscapes, representations and designs}

'Landscape' is defined as 'an area, as perceived by people, whose character is the result of the action and interaction of natural and/or human factors' (Council of Europe 2000). This definition emphasizes the diversity of landscape materiality; it is a perceived entity, composed of both natural and social components (Jacobs 2004). There are different traditions of landscape design that employ different visual modes and media. Especially the distinction of natural and social components is reflected in the landscape design traditions of both European and American scholars and practitioners (Laurie 1975; MacHarg 1969; Ndubisi 2014; Steiner 2006). These traditions use the concept of 'landscape systemics' (Duchhart 2007; Vroom 2006). These systems are represented in maps, cross-sections and diagrams to illustrate how a given landscape 'works' and what are the drivers of change (Barnett 2013) (Figure 3.1). 


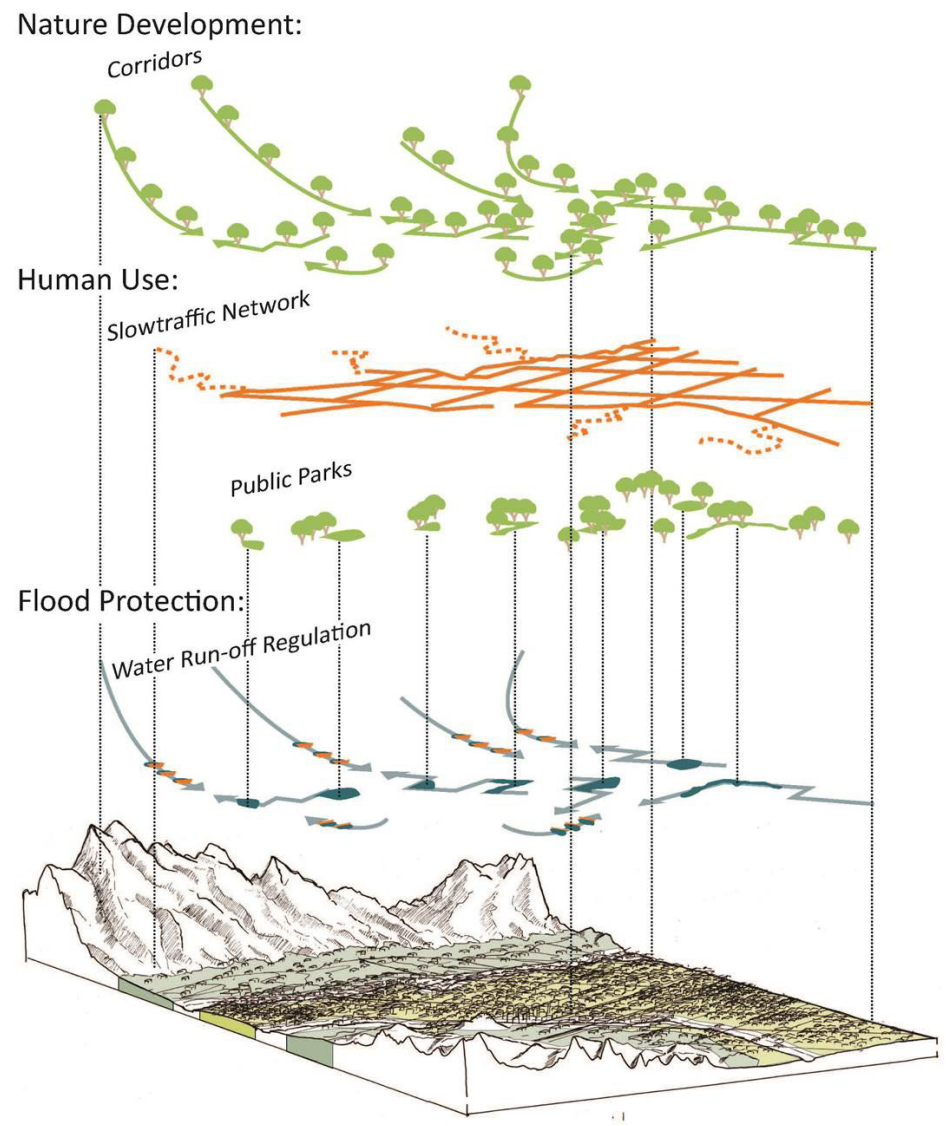

Figure 3.1. Example of schematic visual representation showing landscape systemics (Winder, 2014).

Others conceptualize landscape as a perceived entity. Scholars part of this tradition argue that it is not only important to study how the landscape works, but also how the landscape is experienced (van der Knaap 1997; van Etteger et al. 2016; Wylie 2005). This perception of the real-life physical landscape is not static. It is based on experience (for example, by walking through the landscape in question), education, time of day, season and even our personal mood. It is also influenced by exposure to visual and verbal landscape representations, such as films, paintings or poetry (Cosgrove \& Daniels 1988; Mitchell 2002). This continually changing 
landscape experience is often expressed with atmospheric images (Figure 3.2) or time-lapses.

Some scholars turned to textual metaphors in their search for new methodological foundations to enable them to grasp this diversity of perceived landscape meaning (Bellentani 2016; Duncan \& Duncan 1988; Lindström et al. 2014). By attributing textual qualities to physical landscapes, or to visual representations of landscapes, these 'landscape texts' could be considered as communicative entities. As texts, landscapes acquire authors, readers and narratives (Potteiger \& Purinton 1998; Spirn 1998), which positions the landscape planner or designer as someone who gains (or claims) authorship of the narrative they intend the designed landscape to express. In most cases design representations that have to express such a story require a certain sequence of images, a collage (Figure 3.3) or moving pictures.

However, we would be missing the complexity and multiplicity of landscape designs if we limited our understanding of landscape planning and design to just the implementable design and the story it tells. A 'design' is not always an end product: unrealized design ideas and concepts also have a strong influence on contemporary design culture (Cridge 2015; Morrison 2015). A design is also not a fixed materiality, i.e. a drawing, rendering, 3D model, building or physical landscape (Gough 2016). Instead, designing is a process of creation and establishing authorship that involves the accumulation of knowledge, ideas, emotions and imagination (Cross 2006; Rattenbury 2002). These elements materialize in design representations through various visual techniques and media. Each of these

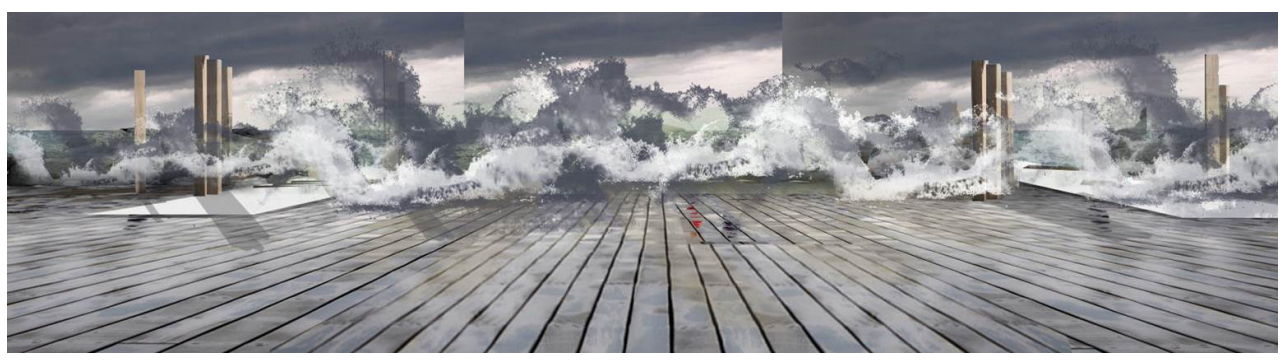

Figure 3.2. Example of atmospherical visual representation showing the potential experience of a flood event (Valcarlos, 2014). 
representations contains specific communicative qualities that influence the planning and design process. For instance, a drawing can be seen as a producing agent or 'ideational catalyst' and not just as a representation of a landscape. Rather, it can depict the capacity of that landscape to be transformed into a desired future state (Corner 1992).

We need to regard all kinds of landscape design representations as important means of visual communication that enable a critical reflection on a landscape design and on the process of that design's creation. This includes expressions of how the landscape works as a system (Figure 3.1) as well as the atmospheric interpretation of sensory perception (Figure 3.2). We argue that theories on semiotics and iconography provide a foundation for an analytical framework with which to address the diversity of design representations and the multiplicity of meanings that can be attributed to them.

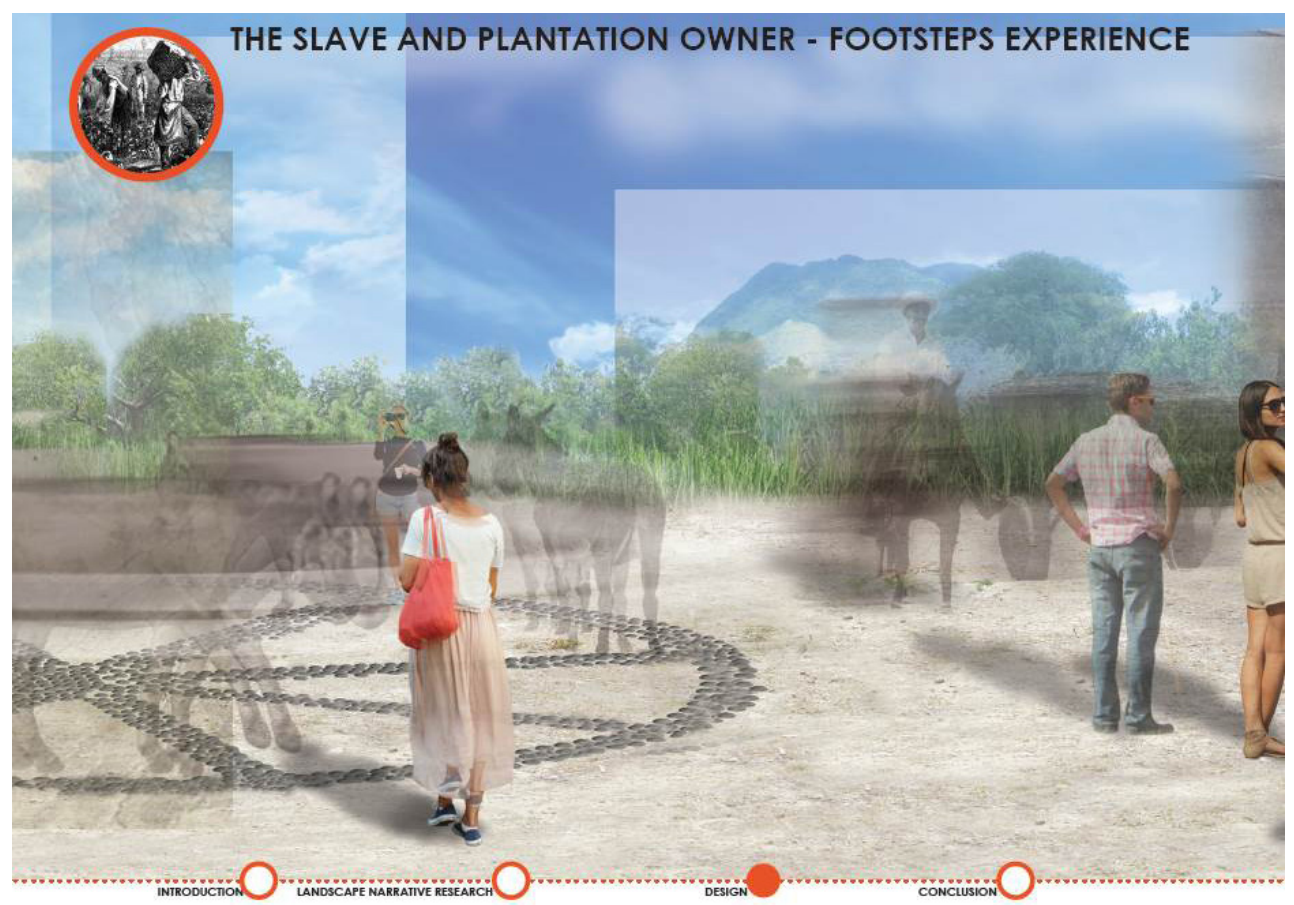

Figure 3.3. Example of a visual representation that shows the historical narrative of a landscape (van Zuiden 2016). 


\section{$\triangle$ \\ 3.3 A lens of semiotics and iconography}

Semiotic theories describe how the meaning of signs is constructed and interpreted through a process of semiosis (Jappy 2013). Peirce (1958), as cited by Eco (1976: 15), defines semiosis as an action or influence that involves the cooperation of three components: a sign or representamen, its object and its interpretant. Semiosis is always a triadic function and therefore not resolvable into actions between pairs. According to Eco (1976), following Peirce, 'everything' has the potential to be a sign as long as it has a substantial significance to something else for someone. Visual representations of landscape designs, such as Figures 1-3, can thus be considered as signs. They also contain a great number of other signs within themselves, such as their visual elements, techniques and symbols. Even the planner's or designer's intention and knowledge embedded in the design can considered to be signs. As a whole, a landscape design and its many representations consist of multiple sign systems.

In this paper we are interested in the relationship between the design image (expression) and the design properties it refers to (content) as well as how this signifying relationship can - potentially - be interpreted in practice through communication. We adopt the hybrid theory of semiotics developed by Umberto Eco (Eco 1976) to study this relationship. In Eco's theory, dyadic signification (Hjelmslev 1953) is embedded within a triadic (Peirce 1958) communication process (Chandler 2007). In this way, dyadic 'semiology' offers tools for defining categories and a semiotic vocabulary for a content analysis focused on the informed, descriptive meaning of landscape design representations: what kind of image it is, what it looks like and what kind of information it shows. Triadic semiotics connects these informed, descriptive meanings to possible interpretations in a process of communication. In this way we embed the dyadic relationship of expression and content within a triadic system of interpretative sign production (Figure 3.4). 


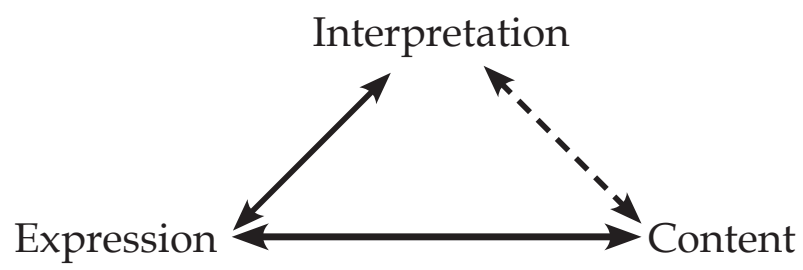

Figure 3.4. Hybrid Semiotics (based on Eco): dyadic theory of Hjelmslev embedded in the triadic logic of Peirce (own figure).

The triadic logic of interpretation is not a closed process where certain content is to be transferred via an expression to establish a definitive interpretation. The interpretation of a sign forms a new 'sign within the mind' which refers to another object, which in turn may refer to something else (Eco 1976). For example, a representation of a dike construction refers to the physical flood defence structure it represents. For an engineer, this image triggers a process of additional significations related to structural capacity, measurements and building materials. For a city planner, that same image signifies real estate exploitation, zoning regulations and budget restraints. This string of connotations, potentially endless, is known as a signifying chain.

Understanding the workings of signifying chains is necessary to study the semiotic complexity and possible interpretations of landscape design representations. This complexity arises along three lines of logic: semantics, syntactics and pragmatics (Morris 1938). Semantics is defined as the study of the abstract relationship between signs and the objects they signify. Syntactics entails the relationship between signs, whilst pragmatics involves the study of the origin and use of signs in relation to their interpreters (Allwood 1981). The interplay between these three concepts constitutes the complexity of meaning-making processes in practice.

Whereas the semantics and syntactics of landscape design representations can be systematically analysed using categories derived from the dyadic semiotics of Hjelmslev - as explained later in this paper - the analysis of pragmatics requires 
an additional interpretive framework. As researchers, we are neither the authors nor the target group for design representations. Analysing the pragmatics of design representations requires that we interpret and reconstruct signifying chains outside the original context of the image. By collecting and sampling image data with a specific motif in mind - semiotic complexity - the researcher's intuition becomes part of the method (Müller 2011: 286). Knowledge of the researcher regarding design processes and competitions, public participation, climate change and flood defence projects determines the contextualization of the image data. Similarly, iconographers interpret historical works of art by reconstructing the historical context of the artwork (Panofsky 1955). The iconographic tradition provides an insight into the conventionality - and predictability - of the way in which different types of meaning are constructed under different circumstances.

Following Van Leeuwen (2001), we distinguish three types of 'pragmatic' meaning produced by the semantics and syntactics of signifying chains: 'representational meaning', 'iconographical meaning' and 'iconological meaning'. Representational meaning refers to the most direct expressional and factual meaning that can be derived from everyday experience, stylistic conventions and mediation by different visual technologies (van Leeuwen 2001). This type of denotative meaning constitutes an image's 'objectivity' (Barthes 1977: 19). It is the most commonly shared universal type of meaning based on practical everyday experience sensitive of social and cultural traditions (Panofsky 1955). Iconographical meaning is based on generally accepted conventions: signs that are able to work together in a conventional manner. For instance, smiling figurines in a photomontage signify a safe, happy place, whilst dark clouds on the horizon above a dike structure could signify the looming danger of climate change. Furthermore, through intertextuality (Rose 2012: 191), it is possible to relate an image to its context, whether this is, for example, a design proposal, a design competition or issues of climate change. 
Finally, iconological meaning is derived from unconventional symbolism used by specific social groups (van Leeuwen 2001). These types of signs are used consciously or unconsciously, yet signify the underlying principles of their creation. Specific signs, reserved for specific disciplines, might lead others to overor misinterpret them. Not to claim we can predict 'good' interpretations we could at least ascertain which interpretations are 'bad' (Eco 1992: 52). For landscape planners and designers, the use of certain perspectives and colour schemes or even the inclusion of a flock of birds as a finishing touch, might seem conventional, yet could invoke unintended interpretations for stakeholders from other disciplines (Raaphorst et al. 2017).

\subsection{Analytic framework}

The analytical focus of this paper is the image stage (Rose 2012) and therefore on the relation between the visual representation and the content it refers to. An analytic framework is necessary to code and categorize design representations in a way that facilitates a systematic content analysis of semantics and syntactics. We zoom in on the dyadic relationship of content-expression through the Deleuzian interpretation of Hjelmslev's semiotic framework (Hjelmslev 1953). This framework provides analytical categories with which to describe 'expression' and 'content' in ways that resonate with existing theories of map making (MacEachren 1995) and cartographic 'semiology' (Bertin 2011) that are well-known within the disciplines of landscape planning and design.

Hjelmslev was a linguist who developed dyadic semiotics into a form of logistics that is partly compatible with Peircean triadic logic (Eco 1976; Parret 1984). In total, Hjelmslev describes six analytical categories. Each so-called 'plane', the plane of expression and the plane of content, has its own form, substance and matter (Deleuze \& Guattari 1987; Hjelmslev 1953; Taverniers 2008). Deleuze and 
Guattari operationalized Hjelmslev's linguistic framework for the poststructuralist semiotic analysis of non-linguistic signs, such as natural and social phenomena. They argue that the relationship between content and expression is not one of correspondence, as it is understood within Eco's structuralist semiotic theory, but rather one of reciprocal forms of semiotic organization (Deleuze \& Guattari 1987: 50). For example, just as colour needs a certain shape to express itself, each of the six categories cannot exist without the other. They are fundamentally intertwined. We follow the reciprocal conceptualization of Deleuze and Guattari, but partly based on Chandler (2007), we translate the six categories into categories suitable for a visual analysis of landscape design representations (see Table 3.1). These translated categories are based on literature from art history (Acton 1997; Taylor 1981), film studies (Metz 1973), environmental design (Lang 1987) and social semiotics (Kress 2009).

Both categories of matter are theoretical and embody the potential of content and expression (Deleuze \& Guattari 1987; Taverniers 2008). Here these categories determine the scope for the conceptualization of content and expression of landscape design representations within the context of participatory planning and design processes of multifunctional flood defence solutions. Since we define

Table 3.1. Operationalization of Hjelmslev's categories for landscape design representations.

\begin{tabular}{|c|c|c|c|}
\hline $\begin{array}{l}\text { Operationalized } \\
\text { category }\end{array}$ & Description & Category & Plane \\
\hline Medium & $\begin{array}{l}\text { The physical materials that carry the } \\
\text { visualizations }\end{array}$ & Substance & \\
\hline Mode & $\begin{array}{l}\text { The visual techniques, perspectives, scale, } \\
\text { and styles }\end{array}$ & Form & Expression \\
\hline Visual Culture & $\begin{array}{l}\text { Array of visual means available: colors, } \\
\text { shapes, projections }\end{array}$ & Matter & \\
\hline Subject Matter & $\begin{array}{l}\text { Landscape components: soils, climate } \\
\text { systems }\end{array}$ & Matter & \\
\hline Formulation & Message, narrative, intent & Form & Content \\
\hline Knowledge & Data, information, measurements, statistics & Substance & \\
\hline
\end{tabular}


this scope as such, we only analyse the empirical categories, namely 'knowledge', 'formulation', 'mode' and 'medium', for the visual content analysis. The scope and context defined by the 'matter' categories are used to contextualize the images during the iconographical interpretation.

The expression categories of 'medium' and 'mode' are analysed visually akin to compositional interpretation (Taylor 1981). We review the source material by, for instance, looking at what technique, perspective and visual style as well as which media were used to present the images. The content categories of 'knowledge' and 'formulation' are analysed by looking at the 'anchorage' (Barthes 1977) of the images: their original descriptions, titles, subscripts, legends and references in the text.

Each of the four empirical categories has its own sign function and constitutes a semiotic component of the image as a sign system. These components evoke an arrangement of sign-systems that influence the design representation communication process. This arrangement is determined by syntactics; the relationships between signs. For this study, we identify and introduce three important syntactic relationships to conceptualize the way design representations are organized, namely interactivity, readability and validity (Figure 3.5).

Interactivity is the primary relation between the medium and the mode. For example, a sketch (mode) on paper placed on a table (medium) enables active participation because participants can, and may feel invited to, modify the drawing. Yet, that same sketch (mode) projected on a projection screen (medium) creates distance and prohibits openness and accessibly. Readability is the primary relation

\begin{tabular}{|c|c|c|c|c|c|}
\hline & & \multicolumn{3}{|c|}{ Interactivity } & \\
\hline & & Matter & Substance & Form & \\
\hline & Expression & Visual Culture & Medium & Mode & $\uparrow$ \\
\hline Sign & & & & & Readability \\
\hline & Content & Subject Matter & Knowledge & Formulation & $\downarrow$ \\
\hline
\end{tabular}

Figure 3.5. Syntactic relationships of validity, readability, and interactivity (own figure). 
between the mode and the formulation. A 3D model from an eye-level perspective (mode) shows the height differences of landscape elements (knowledge) of a final design (formulation) in a visual way that adheres to human perception and scale. A height map with a 2D top view perspective (mode) presents the same knowledge, but presents it in a quantitative and mathematical way. Finally, validity entails the primary relation between knowledge and formulation. For example, whether the physical properties of a dike construction (knowledge) are characterized as a conceptual design (formulation) or as an operational design (formulation) validates the representation of that dike construction as either design principles or blueprints, respectively. These relationships, in turn, influence each other as part of a reciprocally functioning sign system.

If we connect Eco's hybrid theory of semiosis with Hjelmslev's logistic theory of content and expression (Figure 3.4) and operationalize that theory using the categories and syntactic relationships as defined in Table 3.1 and Figure 3.5 and then position iconographic theory in relation to pragmatics, syntactics and semantics, we arrive at the analytic framework of Figure 3.6.

The semantics and syntactics of the images, based on the four empirical categories derived from Hjelmslev, are analysed through a visual content analysis. The representational and iconographical pragmatics, defined by the two 'matter' categories of Hjelmslev, are analysed through an iconographical interpretation. The iconological layer is the most subjective and interpretive type of meaning. This type of meaning ideally consists of a synthesis of different political, social and cultural sources of information relevant to the images, such as other texts as well as verbal sources such as interviews (Müller 2011). In this paper, the focus lies on the image and the visual content that can be derived from that image. Therefore, we focus only on the representational and iconographical interpretations of the semantic and syntactic properties and on the syntactic relationships between the designated categories. 


\section{Representational Iconographical}

\section{Pragmatics Interpretation}

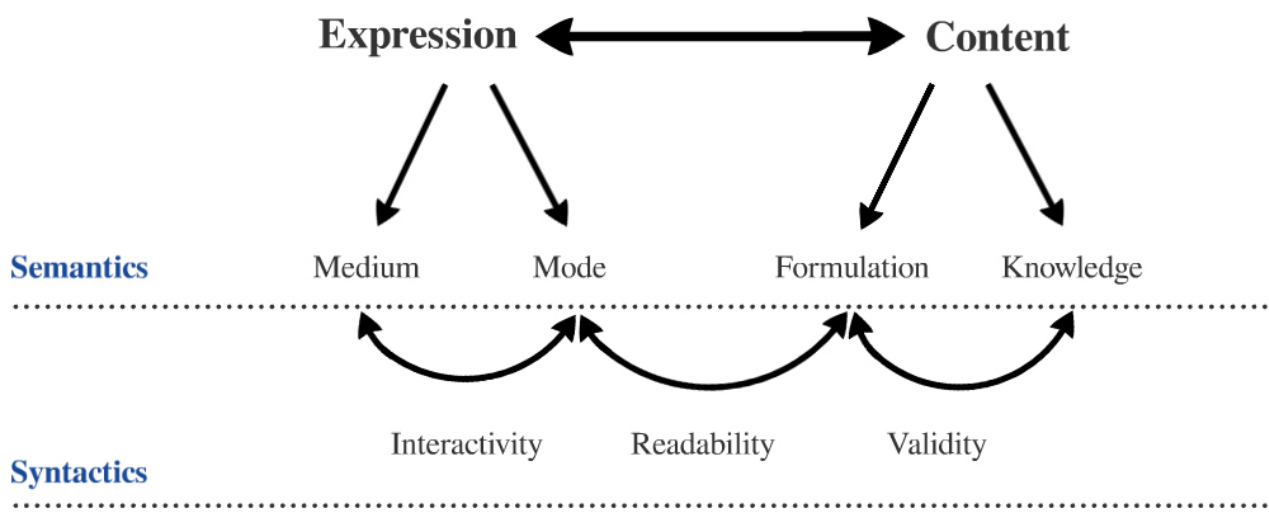

Figure 3.6. Analytic framework: combining iconography with Peirce, Eco \& Hjelmslev (own figure).

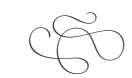

\subsection{Images, codes and interpretation}

The analytic framework presented in Figure 3.6 provides the basis for a visual study that demonstrates how semiotic complexity can be identified for further, qualitative analysis. This method consists of two parts: (1) a quantitative visual content analysis and (2) a qualitative iconographical interpretation. First, we will describe the image material that was collected for our study. 


\section{Design projects}

We applied our method to two submissions for the Rebuild by Design (RbB) competition. Design competitions, such as $\mathrm{RbD}$, result in well-documented collections of visual representations from various design phases (Chupin 2011). Furthermore, the multifunctional and participatory nature of $\mathrm{RbD}$ provides a good example for analysing the diversity of visual techniques used in the context of such projects. RbD began as a transdisciplinary participatory design competition, modelled after the 'Dutch approach', that advocates collaborative planning and design solutions on a regional scale (de Jonge 2009; Sijmons 1990; Vroom 1990).

The goal of $\mathrm{RbD}$ was to arrive at multifunctional landscape solutions in the greater metropolitan New York area in the aftermath of hurricane Sandy in 2012. Since then it has become an innovative process capable of generating implementable solutions for a more resilient region (Ovink \& Boeijenga 2016). In 2013, the design brief (Rebuild by Design 2013) stated that project teams were required to combine international, regional and local knowledge to, for example, achieve a comprehensive SWOT analysis of the area, design regionally applicable solutions that have the public support of local communities and federal agencies and strengthen the relationship between local and regional stakeholders.

Two out of the seven winning RbD proposals were selected for analysis based on their scale, spatial and collaborative complexity as well as their specific flood challenge type: one river area and one coastal area proposal. The project areas are of a regional scale, i.e. land use consists of both natural and urban areas. Consequentially, several economical and socio-political stakeholder groups are involved in the projects. A substantial part of the project teams consist of Dutch design firms that are well accustomed to the regional and collaborative approach that is advocated by RbD. The first proposal, the New Meadowlands plan (MITCAU et al. 2014), addresses the area of the New Jersey Meadowlands. This area is a 30.4 square-mile $\left(79 \mathrm{~km}^{2}\right)$ district, contains a preserved river wetland area and encompasses parts of fourteen municipalities and two counties. This proposal was 
made by the MIT-CAU project team, which consisted mainly of MIT-CAU and Dutch design firms ZUS and Urbanisten. The second proposal, the Living With the Bay plan (Interboro 2014), addresses Nassau county's south shore. This area is a 155 square-mile $\left(250 \mathrm{~km}^{2}\right)$ district, contains Long Island, part of Jones Beach Island, marshlands and many different villages and towns. The proposal was produced by the Interboro team, which consisted mainly of NY-based Interboro and Dutch design firms $\mathrm{H}+\mathrm{N}+\mathrm{S}$, BoschSlabbers and Palmbout. Both proposals deal with a comparable diversity of landscape areas and stakeholder configurations yet the teams' research approaches differ concerning the use of quantitative and qualitative data. This difference in approach enables us to compare different types of content within a similar design context. For instance, the MIT-CAU project strongly emphasises statistical data, whilst the Interboro team uses mostly qualitative sources.

An inventory of the visual material produced during these two projects resulted in a database of 759 images: 203 images of the MIT-CAU project and 556 images of the Interboro project. These images are derived from different sources, such as presentation boards, project reports, slide show presentations and handdrawn sketches from the designers' archives. However, not all the images that were produced were made public. For instance, the majority of hand drawn sketches are often discarded and destroyed once a design leaves its analytical and conceptual stages. Therefore, counting the absolute number of maps, sketches and photomontages does not result in a totally valid identification of the preference for certain modes or media during a particular phase in the design process. However, it does tell us what kind of visual materials were produced to communicate particular kinds of landscape design content in the context of a design competition. In the following section we describe the methods used to analyse the images developed during these two projects. 


\section{Visual content analysis}

In this paper, we build on the work of several scholars (Bell 2001; Kress \& van Leeuwen 1996; Rose 2012) who argue that a visual content analysis, focused on both the content and expression of images, can be applied for the quantitative analysis of qualitative semiotic materials. Content analysis stems from a 'long and welldeveloped tradition of dealing quantitatively with qualitative data (...) where the issue is one of counting the frequency and sequencing of particular words, phrases or concepts found in the data.' (Miles et al. 2014: 43). When performing a visual content analysis the researcher also counts and analyses how frequently certain visual codes occur in a defined set of sample images (Rose 2012). However, the semiotic nature of our approach requires a strong focus on not just the occurrence, but also on the co-occurrence of visual codes to determine the syntactic qualities of the material. The question, therefore, is not how often a certain code occurs, but rather how often that code occurs in combination with other codes.

A content analysis involves the managing of a large collection of data due to the number of images, and/or the number of quotations, codes, and notes attributed to those images. For this reason qualitative researchers are encouraged to use computer software to facilitate an efficient, organized analytical process (Lewins \& Silver 2007; Peters \& Wester 2007). For our analysis, we used Atlas.TI, a software package that allows the coding and subsequent analysis of both written and visual data.

Categories, variables, and codes. Content analysis involves the classification of data into categories. Categories need to be explicitly defined into variables and values to enable a consistent study of content (Bell 2001). In our case, the categories relate to the empirical semiotic components of media, mode, knowledge and formulation. Each of these categories is defined through variables describing visual qualities or dimensions (Table 3.2).

For the expression categories, the technology and type of the medium (Kress \& van Leeuwen 1996), the graphical techniques, spatial projection, perspective, 
Table 3.2. Categories and variables identified from Kress \& van Leeuwen (2006), Taylor (1981), Acton (1997), Lawson (2006), Simonds (1998), and Lang (1987).

\begin{tabular}{ll|ll}
\hline Category & Variable & Category & Variable \\
\hline Medium & Technology & Formulation & $\begin{array}{l}\text { Analytical } \\
\text { Conceptual } \\
\text { Type }\end{array}$ \\
& Perspective & & Operational \\
& Spatial projection & Knowledge & Physical subsystem \\
& Graphical technique & & Socio-economical subsytem \\
& Explanational & & Prodedural \\
& Style & & \\
& Scale & & \\
\hline
\end{tabular}

scale, explanational elements and style of the mode (Acton 1997; Taylor 1981), are all considered as variables. For the content categories, the formulation type relates to the design stage (Lawson 2005; Simonds 1998) and can be generally divided into analytical, conceptual or operational stages. Knowledge variables describe the different subsystems of the landscape (Lang 1987) or describe the design process itself. The values of these variables consist of elements that belong to the same class. For instance, values of the medium variable 'type' consist of book, flyer or projector screen, whilst the mode variable 'technique' consists of values such as sketch, photomontage, map and 3D model. The conceptual formulation has values such as 'design principles', 'design impression' or 'strategy', whilst the knowledge of the physical subsystem contains values such as 'biodiversity', 'soil', 'elevation' or 'climate change'.

Our content analysis involves three rounds of coding using an emergent coding strategy. By using such a strategy, the researcher becomes immersed in the data and creates a code list 'on the fly' by writing down the codes as they are encountered. In our study, these codes were grouped, evaluated and compared with existing literature on visual semiotics, iconography and landscape design. The material was revisited after the first coding round to ensure that codes, which emerged later on 
in the dataset, were applied consistently to the entire dataset. A third round was done as final verification of the coding scheme. This coding scheme constitutes the apparent description of visual materials: the semantic and syntactic codes.

A semantic code refers to an apparent object. It constitutes 'what we see' and can be determined with relative ease. Examples of semantic codes are media. We can easily determine whether an image comes from a book, poster or projection screen. Certain visual techniques can be distinguished as well, such as a 3D model, a satellite image or a photomontage. However, the distinction between a sketch and a drawing, or a design plan and a design concept, is more open to interpretation. These syntactic codes are more ambiguous and emerge during several rounds of coding.

In contrast to semantic codes it is more difficult to define independent variables or mutually exclusive values for syntactic codes. Syntactic codes are more dependent on pragmatics and are therefore of a more interpretative nature. Bell (2001) points to the work of Kress and Van Leeuwen in explaining how certain theoretically informed syntactic concepts can be simplified into variables and values. For instance, Kress and Van Leeuwen (1996: 256) define a variable 'modality' - which compares to the variable 'style' in our coding scheme - as 'the degree to which certain means of pictorial expression (colour, representational detail, depth, tonal shades, etc.) are used'. This variable is defined as a range. Its values are a depiction of the degree to which that variable is present. For example, Bell (2001: 30) defines three values of 'modality': high, medium and low sensory modality.

In our study, we propose three general definitions of style: atmospheric, realistic and schematic. With an atmospheric style, the image contains a soft color palette, little contrast and/or a 'washed out' effect. The image triggers certain feelings or emotions related to the perception and experience of the proposed landscape or area. With a realistic style, the image resembles a realistic view of the landscape. It uses natural colors that directly relate to the physical reality. Photographic components can also be included and little use is made of visual or artistic filters. 
With a schematic style, the image contains a lot of hard contrasts. A strong color palette is used and there is little resemblance to the physical landscape. The image often contains numerals, lines and/or legend items.

Another example of a syntactic variable is that of formulation. In landscape architecture (Simonds 1998) and in planning and design disciplines in general (Lawson 2005), the design process can generally be categorized into three phases: the analytical, the conceptual and the operational. In the analytical phase, design representations show current landscape characteristics as objectively as possible. These representations aim to illustrate how a given landscape 'works', what are the drivers of change and what opportunities, threats and strong and weak points can be defined. In the conceptual phase, design representations show the main characteristics of the possible future state of a landscape, including eventual underlying principles of change. Conceptual images are of a visionary character and relate to desired or feared future situations. In the operational phase, design representations show the desired future state as it will be realised through the implementation of specified interventions. Operational images have a level of detail that fits the purpose of bringing across the message: 'These are the changes to be made to the existing landscape and this is how it will look when the proposed transformations have been realized'.

\section{Iconographical Interpretation}

We can select images based on different kinds of semiotic complexity by looking at the co-occurrence of different medium, mode, knowledge and formulation codes. For example, the modal complexity might warrant further analysis if more than one visual technique, style or scale is present in the mode category. Similarly, several knowledge codes might indicate a technically complex design, whilst different co-occurring formulation codes can produce images that are ambiguous in their message. We can also select images based on their syntactic relationships by 
looking at the co-occurrence between categories, e.g. how frequently combinations of mode and formulation codes occur.

The goal of the iconographical interpretation is to describe the syntactic relationships of the selected images and embed those relations in the pragmatics of their social context using representational and iconographical layers of meaning (Müller 2011). The representational layer consists of the semantic and syntactic codes that emerge from visual content analysis: the types of knowledge, formulation, visual modes and the media that are used. The iconographical layer is described through intertextuality. This includes relating the different semantic and syntactic codes to each other 'internally' and arriving at the syntactic relationships of validity, readability and interactivity. It also includes relating the image 'externally' to other design representations that are part of the design proposal, as well as to the context of both a participatory design process and the $\mathrm{RbD}$ competition.

\section{$\Delta$}

\subsection{Demonstrating the framework}

We demonstrate our analytical framework in two phases. First, we give a semantic and syntactic account of the visual content of the image data. This overview provides us with a general understanding of the images used in the RbD competition in terms of semiotic complexity. Second, we will describe the representational and iconographical meaning of three images to demonstrate how the syntactic relationships of validity, readability and interactivity can be interpreted.

\section{Visual content analysis}

Semantic and syntactic coding scheme. The three coding cycles of the visual content analysis resulted in the coding scheme of Table 3.3, which shows the categories, variables and occurrence of codes. These categories and codes form 
the semiotic vocabulary within the visual database. The 'medium' category has two variables: technology (analog or digital) and type. These codes define the materiality of the medium and the way the audience can interact with the image. Most of the images collected are digital and derived from the digital publication of the project book. The Interboro project team also provided digital presentation documents (152 images) and a presentation board (containing 42 separate images). The MIT-CAU project team produced a brochure (39 images) and booklet (5 images). Their presentation board consisted of only one image and we will address this later in this section.

The 'mode' category consists of variables that relate to composition and technique, namely perspective, spatial projection, graphic technique, style, scale and explanational elements. In both projects, the majority of the material consisted of maps, or drawings of maps, as well as photomontages, photographs and models. Most images have a schematic style (537), while atmospheric (106) and realistic (151) styles are used to about the same extent. The images show a slight tendency to focus on the regional scale with local and area scales following closely. Most explanational elements are textual: titles, captions and textboxes occuring most frequently.

The 'formulation' category consists of analytical, conceptual and schematic variables. Most of the material consists of conceptual (396) and analytical (308) images, whilst only a few images (55) have an operational formulation. The 'knowledge' category is divided into codes relating to the physical subsystem (mostly flood and water related codes), socio-economical subsystem (e.g. land use and infrastructure related codes) and procedural knowledge, e.g. about the phasing of the plan and the design process itself.

Syntactic co-occurrence analysis. The complexity of medium, mode, formulation and knowledge, as well as the semiotic complexity of the image can be described in terms of internal syntactics by looking at the frequency with which code combinations occur. For example, the co-occurrence of different style and formulation codes can help to assess the visual ambiguity of an image, e.g. whether 
Table 3.3. Coding scheme and code occurrences for the categories, variables, and values used in the visual content analysis of landscape design representations for each RbD project.

\begin{tabular}{|c|c|c|c|c|}
\hline Category & Variable & Value & MIT-CAU & Interboro \\
\hline & & & \# occ. & \# occ. \\
\hline \multirow[t]{16}{*}{ Medium } & Technology & Analog & 38 & 12 \\
\hline & & Digital & 160 & 536 \\
\hline & Type & Board & 1 & 42 \\
\hline & & Book & 107 & 344 \\
\hline & & Booklet & 5 & 0 \\
\hline & & Brochure & 39 & 0 \\
\hline & & Card game & 1 & 2 \\
\hline & & Exhibition & 1 & 0 \\
\hline & & Flyer & 0 & 0 \\
\hline & & Painting & 1 & 0 \\
\hline & & Paper & 4 & 8 \\
\hline & & Poster & 1 & 0 \\
\hline & & Presentation & 0 & 152 \\
\hline & & Printout & 0 & 0 \\
\hline & & Projector screen & 1 & 0 \\
\hline & & Transparent paper & 5 & 4 \\
\hline \multirow[t]{19}{*}{ Knowledge } & Physical subsystem & Biodiversity & 11 & 22 \\
\hline & & Elevation & 0 & 22 \\
\hline & & Flood & 39 & 93 \\
\hline & & Soil & 7 & 34 \\
\hline & & Water: climate change & 4 & 7 \\
\hline & & Water: dike structure & 4 & 21 \\
\hline & & Water: management & 0 & 20 \\
\hline & & Water: system & 12 & 28 \\
\hline & Socio-economical subsystem & Demography: density & 3 & 6 \\
\hline & & Demography: income & 2 & 6 \\
\hline & & Demography: vulnerability & 9 & 16 \\
\hline & & Geography: municipalities & 1 & 6 \\
\hline & & Geography: watersheds & 0 & 8 \\
\hline & & Infrastructure & 31 & 47 \\
\hline & & Land Use & 115 & 259 \\
\hline & & Public Sentiment & 1 & 9 \\
\hline & Procedural & Design process & 10 & 26 \\
\hline & & Institutional organization & 2 & 4 \\
\hline & & Phasing & 5 & 40 \\
\hline
\end{tabular}




\begin{tabular}{|c|c|c|c|c|}
\hline Category & Variable & Value & MIT-CAU & Interboro \\
\hline & & & \# осc. & \# осс. \\
\hline \multirow[t]{35}{*}{ Mode } & Perspective & Bird's eye & 53 & 167 \\
\hline & & Eye-level & 10 & 50 \\
\hline & & Elevated eye-level & 20 & 41 \\
\hline & & Front view & 15 & 46 \\
\hline & & Oblique & 3 & 9 \\
\hline & & Top view & 89 & 207 \\
\hline & Spatial projection & 3D model & 9 & 21 \\
\hline & & Cross section & 30 & 61 \\
\hline & & Map & 78 & 235 \\
\hline & & Perspective drawing & 7 & 0 \\
\hline & Graphical technique & Aerial photograph & 4 & 7 \\
\hline & & Collage & 4 & 0 \\
\hline & & Drawing & 41 & 135 \\
\hline & & Engraving & 1 & 0 \\
\hline & & Graph & 8 & 8 \\
\hline & & Infographic & 10 & 0 \\
\hline & & Photograph & 16 & 44 \\
\hline & & Photomontage & 22 & 59 \\
\hline & & Sattelite image & 8 & 47 \\
\hline & & Sketch & 30 & 6 \\
\hline & & Model & 20 & 98 \\
\hline & Explanational & Caption & 48 & 104 \\
\hline & & Compass & 0 & 1 \\
\hline & & Landforms & 38 & 149 \\
\hline & & Landmarks & 5 & 1 \\
\hline & & Legend & 22 & 44 \\
\hline & & Text box & 32 & 180 \\
\hline & & Toponyms & 20 & 47 \\
\hline & & Title & 116 & 349 \\
\hline & Style & Atmospheric & 30 & 76 \\
\hline & & Realistic & 35 & 116 \\
\hline & & Schematic & 137 & 400 \\
\hline & Scale & Local (large) & 64 & 155 \\
\hline & & Area (medium) & 56 & 155 \\
\hline & & Region (small) & 77 & 204 \\
\hline
\end{tabular}




\begin{tabular}{lllrr}
\hline Category & Variable & Value & MIT-CAU & Interboro \\
\hline \multirow{3}{*}{ Formulation } & & \# occ. & \# occ. \\
& \multirow{3}{*}{ Analytical } & Analysis & 73 & 221 \\
& & Challenge & 53 & 47 \\
& & Documentation & 5 & 13 \\
& & Opportunity & 17 & 2 \\
& \multirow{2}{*}{ Conceptual } & Design concept & 29 & 118 \\
& & Design principles & 29 & 73 \\
& & Design impression & 20 & 69 \\
& & Strategy & 34 & 141 \\
& & Abstract concept & 12 & 19 \\
& & Detailed design & 9 & 10 \\
& Operational & Design plan & 12 & 26 \\
\hline
\end{tabular}

the style 'fits' the type of formulation. Analytical images are mostly visualised in a schematic way to show specific - often quantitative - data, just like operational images show concrete physical interventions, required calculations and maps indicating location in a schematic way.

Table 3.4 shows the co-occurrence of style and formulation codes of the images belonging to the Interboro project. This co-occurrence table can be interpreted in two ways for each variable: out of 287 conceptual images, the majority $(0.74)$ have a schematic style. However, we also see that out of 76 images with an atmospheric style, the vast majority (0.86) also have a conceptual formulation. By comparing both relationships (F/S and S/F), we can derive a relative preferred standard of style and formulation. For example, out of all the images with an atmospheric style, very few (0.04) have an analytical formulation. Out of all analytical images, very few (0.01) have an atmospheric style. It might warrant further investigation if this combination of codes does occur to see whether this was a deliberate choice of the makers and whether it affects the analytical qualities of the image.

Similarly, the visual complexity of the 'mode' category can be assessed by analysing the co-occurrence of different 'mode' codes. For instance, the 
Table 3.4. Co-occurrence table of formulation and style codes of the Interboro project.

\begin{tabular}{|c|c|c|c|c|c|c|c|}
\hline Formulation & Analytical & & Conceptual & & Operational & & Image Total \\
\hline Style & & $\mathrm{F} / \mathrm{S}$ & & $\mathrm{F} / \mathrm{S}$ & & $\mathrm{F} / \mathrm{S}$ & \\
\hline Atmospheric & 3 & 0.04 & 65 & 0.86 & 11 & 0.14 & 76 \\
\hline$S / F$ & 0.01 & & 0.23 & & 0.32 & & \\
\hline Realistic & 70 & 0.60 & 48 & 0.41 & 4 & 0.03 & 116 \\
\hline$S / F$ & 0.30 & & 0.17 & & 0.12 & & \\
\hline Schematic & 179 & 0.45 & 212 & 0.53 & 29 & 0.07 & 400 \\
\hline $\mathrm{S} / \mathrm{F}$ & 0.76 & & 0.74 & & 0.85 & & \\
\hline Image Total & 235 & & 287 & & 34 & & \\
\hline
\end{tabular}

Image totals of formulation are corrected for 'double coding', and thus differ from the sum of related codes of table 3.3.

F/S \# Formulation codes divided by \# images with style code

S/F \# Style codes divided by \# of images with formulation code

combination of visual technique and explanational codes helps to assess the selfexplanatory qualities of the image, i.e. whether certain 'anchorage' is needed to guide interpretation when certain visual techniques are used. Table 3.5 shows the co-occurrence of explanational and visual technique codes of the images belonging to the Interboro project. We found that the most occurring explanational elements are textual: title, text box and caption. When we compared the different visual techniques with the number of explanational codes that occurred, we saw that maps, cross sections and schematic models require the most explanational elements. Sketches contain very few explanational elements: no title or text box and perhaps at the most a caption. Other explanational values, such as land forms, landmarks and toponyms, have an explanatory function in terms of geographical orientation and therefore occur mostly in geographical images such as maps and drawings.

A similar analysis is possible for the content of the image: the co-occurrence of formulation and knowledge codes. This type of analysis shows how several types of knowledge codes are used in combination to support an analytical claim, or the idea behind a conceptual design. The combination of mode and formulation shed light on the preferred visualization choices for specific design phases, whilst 
Table 3.5. Co-occurrence table of technique and explanational codes of the Interboro project.

\begin{tabular}{|c|c|c|c|c|c|c|c|c|}
\hline $\begin{array}{l}\text { Explanational } \\
\text { Technique }\end{array}$ & Caption & $\mathrm{A} / \mathrm{T}$ & Compass & $\mathrm{A} / \mathrm{T}$ & Land forms & $\mathrm{A} / \mathrm{T}$ & Landmarks & $\mathrm{A} / \mathrm{T}$ \\
\hline 3D model & 3 & 0.14 & 0 & 0.00 & 0 & 0.00 & 0 & 0.00 \\
\hline $\mathrm{T} / \mathrm{A}$ & 0.03 & & 0.00 & & 0.00 & & 0.00 & \\
\hline Aerial Photograph & 0 & 0.00 & 0 & 0.00 & 0 & 0.00 & 0 & 0.00 \\
\hline $\mathrm{T} / \mathrm{A}$ & 0.00 & & 0.00 & & 0.00 & & 0.00 & \\
\hline Cross Section & 11 & 0.18 & 0 & 0.00 & 3 & 0.05 & 0 & 0.00 \\
\hline $\mathrm{T} / \mathrm{A}$ & 0.11 & & 0.00 & & 0.02 & & 0.00 & \\
\hline Drawing & 33 & 0.24 & 0 & 0.00 & 43 & 0.32 & 1 & 0.01 \\
\hline $\mathrm{T} / \mathrm{A}$ & 0.32 & & 0.00 & & 0.29 & & 1.00 & \\
\hline Graph & 3 & 0.38 & 0 & 0.00 & 4 & 0.50 & 0 & 0.00 \\
\hline $\mathrm{T} / \mathrm{A}$ & 0.03 & & 0.00 & & 0.03 & & 0.00 & \\
\hline Map & 31 & 0.13 & 1 & 0.00 & 135 & 0.57 & 1 & 0.00 \\
\hline $\mathrm{T} / \mathrm{A}$ & 0.30 & & 1.00 & & 0.91 & & 1.00 & \\
\hline Photograph & 9 & 0.20 & 0 & 0.00 & 8 & 0.18 & 0 & 0.00 \\
\hline $\mathrm{T} / \mathrm{A}$ & 0.09 & & 0.00 & & 0.05 & & 0.00 & \\
\hline Photomontage & 16 & 0.27 & 0 & 0.00 & 0 & 0.00 & 0 & 0.00 \\
\hline $\mathrm{T} / \mathrm{A}$ & 0.15 & & 0.00 & & 0.00 & & 0.00 & \\
\hline Sattelite Image & 10 & 0.21 & 1 & 0.02 & 11 & 0.23 & 0 & 0.00 \\
\hline $\mathrm{T} / \mathrm{A}$ & 0.10 & & 1.00 & & 0.07 & & 0.00 & \\
\hline Schematic Model & 19 & 0.19 & 0 & 0.00 & 12 & 0.12 & 0 & 0.00 \\
\hline $\mathrm{T} / \mathrm{A}$ & 0.18 & & 0.00 & & 0.08 & & 0.00 & \\
\hline Sketch & 2 & 0.33 & 0 & 0.00 & 2 & 0.33 & 0 & 0.00 \\
\hline $\mathrm{T} / \mathrm{A}$ & 0.02 & & 0.00 & & 0.01 & & 0.00 & \\
\hline Image Total & 104 & & 1 & & 149 & & 1 & \\
\hline
\end{tabular}

A/T \# Explanational codes divided by \# images with technique code T/A \# Technique codes divided by \# images with explanational code

the combination of medium and mode indicates whether an image was active of passive in terms of interactivity. We address these syntactic relationships by looking at the pragmatics of the images. 
Legend A/T Text box A/T Title A/T Toponyms A/T Image Total

$\begin{array}{rlrlrlrlr}0 & 0.00 & 5 & 0.24 & 20 & 0.95 & 0 & 0.00 & 21 \\ 0.00 & & 0.03 & & 0.06 & & 0.00 & & \\ 0 & 0.00 & 3 & 0.43 & 5 & 0.71 & 0 & 0.00 & 7 \\ 0.00 & & 0.02 & & 0.01 & & 0.00 & & \\ 2 & 0.03 & 34 & 0.56 & 45 & 0.74 & 1 & 0.02 & 61 \\ 0.05 & & 0.19 & & 0.13 & & 0.02 & & \\ 1 & 0.01 & 54 & 0.40 & 94 & 0.70 & 2 & 0.01 & 135 \\ 0.02 & & 0.30 & & 0.27 & & 0.04 & & \\ 1 & 0.13 & 4 & 0.50 & 6 & 0.75 & 0 & 0.00 & 8 \\ 0.02 & & 0.02 & & 0.02 & & 0.00 & & \\ 30 & 0.13 & 71 & 0.30 & 159 & 0.68 & 47 & 0.20 & 235 \\ 0.68 & & 0.39 & & 0.46 & & 1.00 & & \\ 7 & 0.16 & 5 & 0.11 & 11 & 0.25 & 5 & 0.11 & 44 \\ 0.16 & & 0.03 & & 0.03 & & 0.11 & & \\ 0 & 0.00 & 18 & 0.31 & 38 & 0.64 & 0 & 0.00 & 59 \\ 0.00 & & 0.10 & & 0.11 & & 0.00 & & \\ 10 & 0.21 & 7 & 0.15 & 18 & 0.38 & 0 & 0.00 & 47 \\ 0.23 & & 0.04 & & 0.05 & & 0.00 & & \\ 5 & 0.05 & 38 & 0.39 & 65 & 0.66 & 7 & 0.07 & 98 \\ 0.11 & & 0.21 & & 0.19 & & 0.15 & & \\ 0 & 0.00 & 0 & 0.00 & 0 & 0.00 & 0 & 0.00 & \\ 0.00 & & 0.00 & & 0.00 & & 0.00 & & \\ 44 & & 180 & & 349 & & 47 & & \\ & & & & & & & & \\ & & & & & & \\ & & & & & & \\ & & & & & & \\ & & & & & & & \\ \end{array}$

Iconographical interpretation

Pragmatics. Images can be selected on the basis of the frequency of code cooccurrence and subsequently subjected to an iconographical interpretation to study the images' syntactic relationships of validity, readability and interactivity at the representational and iconographical layers of pragmatics. As an example, we interpret three images based on their semantic and syntactic codes and define their syntactic relationships. 
The validity of an image depends on the way a formulation is related to the knowledge behind it. In the case of Figure 3.7 it was not the combination of codes that made the image stand out, but the lack of a certain combination of codes. The image shown in Figure 3.7 is coded with the variables and values presented in Table 3.6.

Through a representational interpretation of the Figure 3.7 map we can describe the greater metropolitan area of New York and that the University of South Carolina's Social Vulnerability index has been used to map the degree of social vulnerability in this area. Usually, an analytical image that promotes a challenge such as this includes several knowledge codes: vulnerability illustrated by flood scenarios or due to the elevation of the area. This image contains only the demographic vulnerability code. The question is whether this information is self-explanatory in a visual way, or whether it needs textual anchorage to convey its message.

The title and heading given to this image contain the words 'vulnerability index', 'risk' and 'opportunity'. It also makes a strong claim: 66\% of most vulnerable communities live within a half a mile of the flood zone. The flood zone is an important element in this statement. However, a geographical depiction of the flood area is not visible on the map. The validity of the data on social vulnerability is not really in question. However, the statement in relation to the flood zone, and whether $66 \%$ of the population live within that zone, cannot be verified on the basis of the image and its textual anchorage.

A further iconographical interpretation - contextualization - helps to assess the validity of the statement illustrated by the map. The vulnerability index standardizes 'indicators of vulnerability based on data such as income levels, poverty rates, ethnicity, language, and access to transportation. Additionally, on-the-ground fieldwork helped the teams gather knowledge beyond what they could glean from the data alone' (MIT-CAU et al. 2014: 43). We therefore assume this index has sufficient scientific credibility. Through intertextuality we also find 
Table 3.6. Codes attributed to the image of Figure 7. Relevant code signified in the color grey.

\begin{tabular}{ll|ll}
\hline \multicolumn{2}{c|}{ Expression } & & Content \\
\hline Medium & Mode & Formulation & Knowledge \\
Book & Icons \& Symbols & Analytical & Demography: vulnerability \\
& Legend & Challenge & \\
Map & & \\
& Scale: small & & \\
& Style schematic & & \\
& Text & & \\
& Title & & \\
& Top view & & \\
&
\end{tabular}

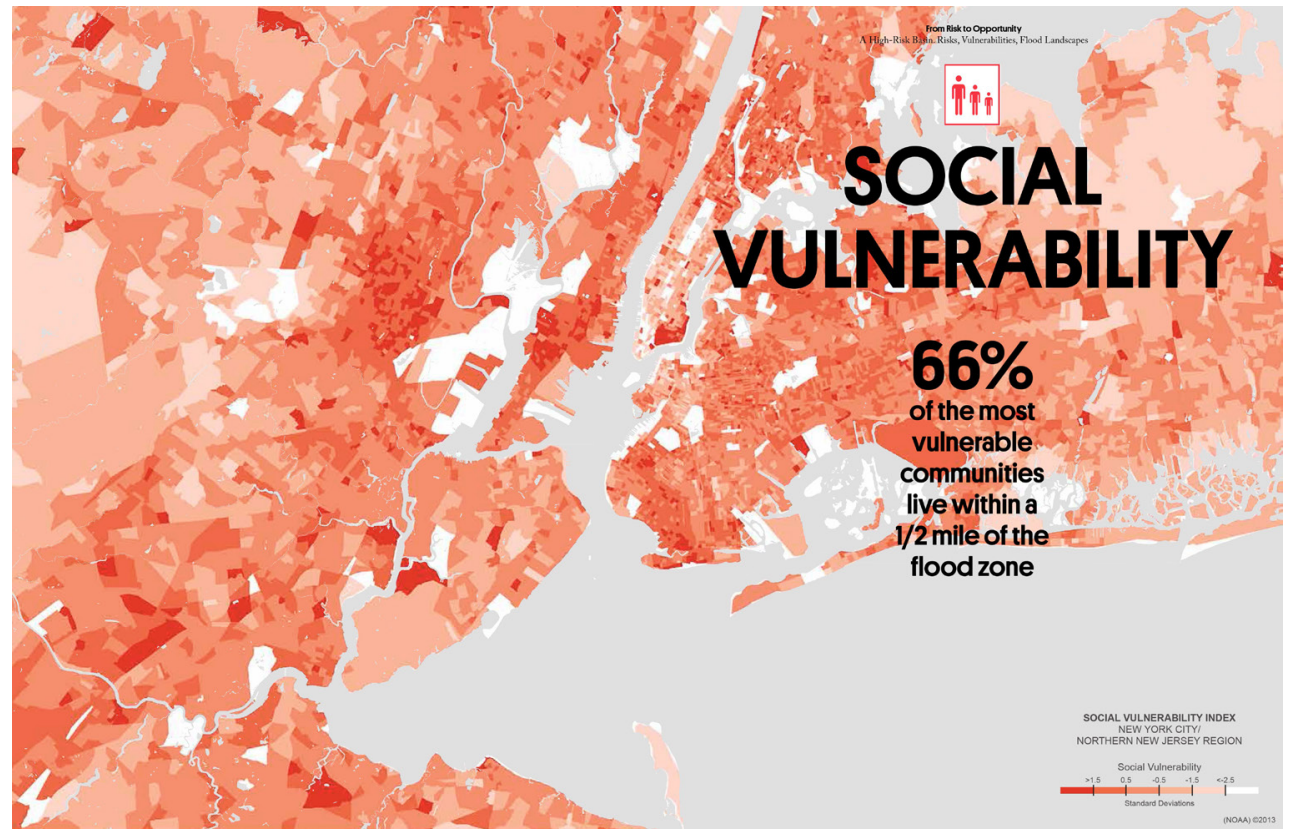

Figure 3.7. Validity: an image with an analytical formulation (MIT-CAU et al. 2014).

that the map is part of a larger regional analysis that also addresses geographic topologies, the flood zone, pollution and vital infrastructure networks. In the context of a design proposal, with the understanding of how regional analysis works, the social vulnerability map can be placed in series with other similar 
maps. This allows the viewer to project the flood zone from the accompanied map in his or her interpretation of the social vulnerability map. This makes a statement such as the one on this map possible without displaying all the information in the same image.

Readability entails whether the mode and the formulation of the image are in coherence. The image in Figure 3.8 was coded with the following semantic and syntactic codes and was selected because of the rare co-occurrence of atmospheric style and analytical formulation (Table 3.7). At a representational level, the image depicts an analysis of coastal areas affected in the flood zone. The atmospherical style is applied to an overlay of a map with a satellite image of a small section of the coast. In combination with a schematic map, this provides an overview of the larger coast area and depicts the most vulnerable locations. The textual anchorage explains that sea levels are expected to rise and that a large number of energy facilities and houses will be at risk if there is a flood event in the future.

At an iconographical level, the atmospherical style adds meaning to the schematic map: the washed out effect of the storm water could connote the severity of the flood. The affected areas are visible in a satellite image so one can see the individual houses. This provides both a strong sense of geographical orientation

Table 3.7. Codes attributed to the image of Figure 8. Relevant codes signified in the color grey.

\begin{tabular}{ll|ll}
\hline \multicolumn{2}{c|}{ Expression } & & Content \\
\hline Medium & Mode & Formulation & Knowledge \\
\hline Bigital & Land forms & Analytical & Flood scenario \\
& Map & Challenge & Land use \\
& Satellite image & & \\
& Style: atmospheric & & \\
& Style: schematic & & \\
& Text box & & \\
& Title & & \\
& Top view & & \\
& Toponyms & & \\
&
\end{tabular}




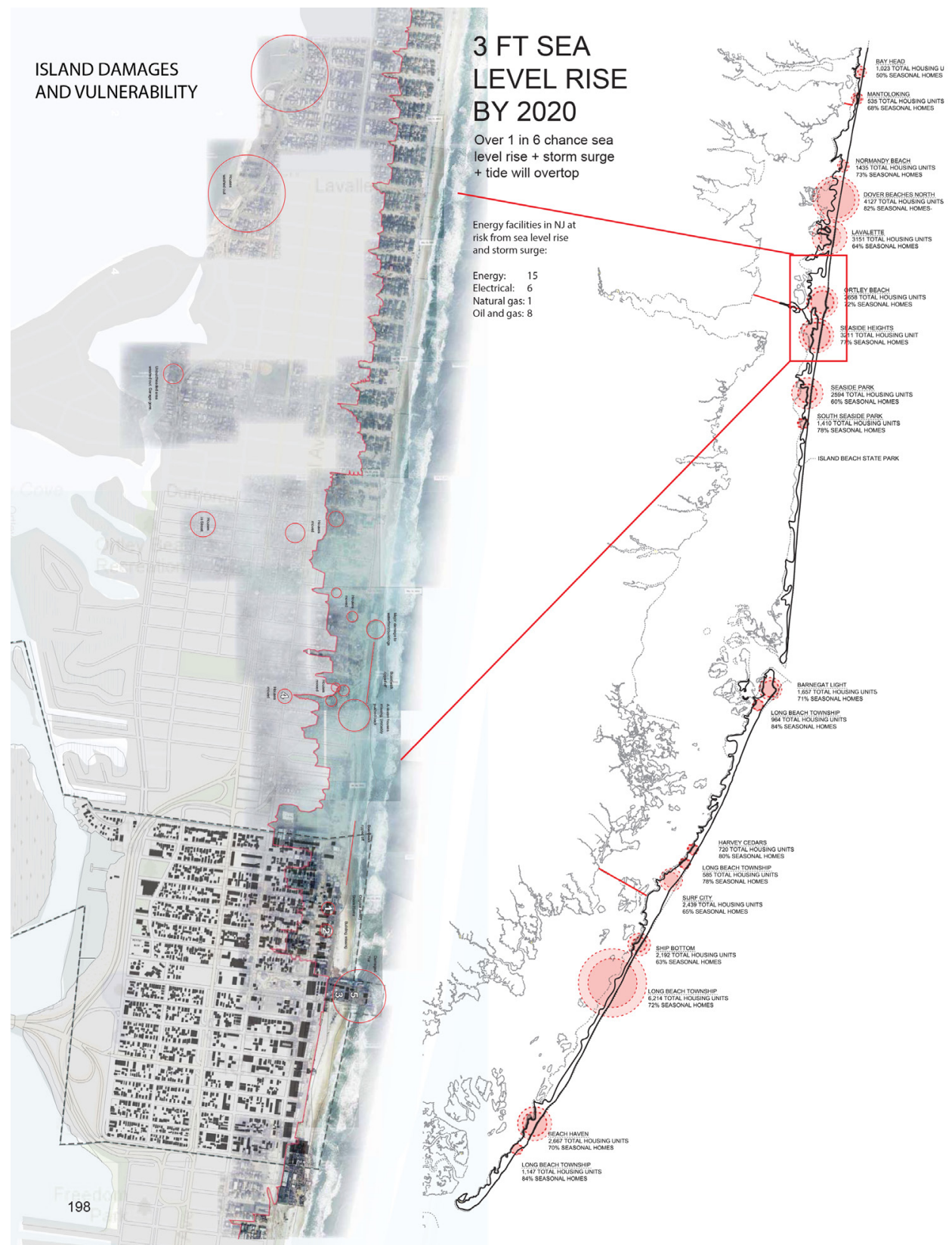

Figure 3.8. Readability: an analytical image with an atmospherical style (Interboro, 2014). 
and an impression of the number of houses likely to be affected. The unaffected areas remain anonymous: they are visible as a schematic map on which one can only distinguish the streets and blocks. The mixture of visual styles therefore directs attention to the affected areas that are shown in more detail.

Interactivity is defined as the relationship between the mode and the medium, i.e. the way the audience is able to interact with an image. The image shown in Figure 3.9 was selected because the co-occurrence analysis of the medium 'board' with the mode 'visual technique' yielded only two codes for the MIT-CAU project (cross section and drawing, Table 3.8), whilst the board material in the Interboro project consists of eight different visual techniques.

At a representational level, the image consists of a design impression of three different types of flood defence landscapes linked together in one strategy: 'protect, connect, grow'. The perspective drawing is placed on top of a cross section showing terrain elevation. The strategy is outlined in a text box in the middle of the poster in relatively small font. In terms of interactivity, the board works on at least two levels of view distance: from afar through the title, concept terms and the design impression, and from up close through the fine print description.

If we analyse the board using an iconographic lens, the accompanied documentation tells us it was presented during an important meeting where the designers were able to comment and offer further explanations of the board. The choice of visual technique and style invites the viewer to interact with not only the board, but also the designers themselves. As a communication device, such a board contains little information in itself, yet establishes a specific kind of interactive communication that triggers and facilitates discussion, embedded within the context of a design presentation.

These three examples of interpreting the validity, readability and interactivity of images illustrate how frequently combinations of semantic and syntactic codes occur can indicate a semiotic complexity that warrants further qualitative study. 
Table 3.8. Codes attributed to the image in Figure 9. Relevant codes signified in the color grey.

\begin{tabular}{ll|ll}
\hline \multicolumn{2}{c|}{ Expression } & \multicolumn{1}{c}{ Content } \\
\hline Medium & Mode & Formulation & Knowledge \\
\hline Analog & Cross section & Design impression & Land use \\
Board & Drawing & Strategy & Infrastructure \\
& Logo & & \\
& Perspective drawing & & \\
& Scale: large & & \\
& Style: atmospheric & & \\
& Text Box & & \\
& Title & & \\
&
\end{tabular}

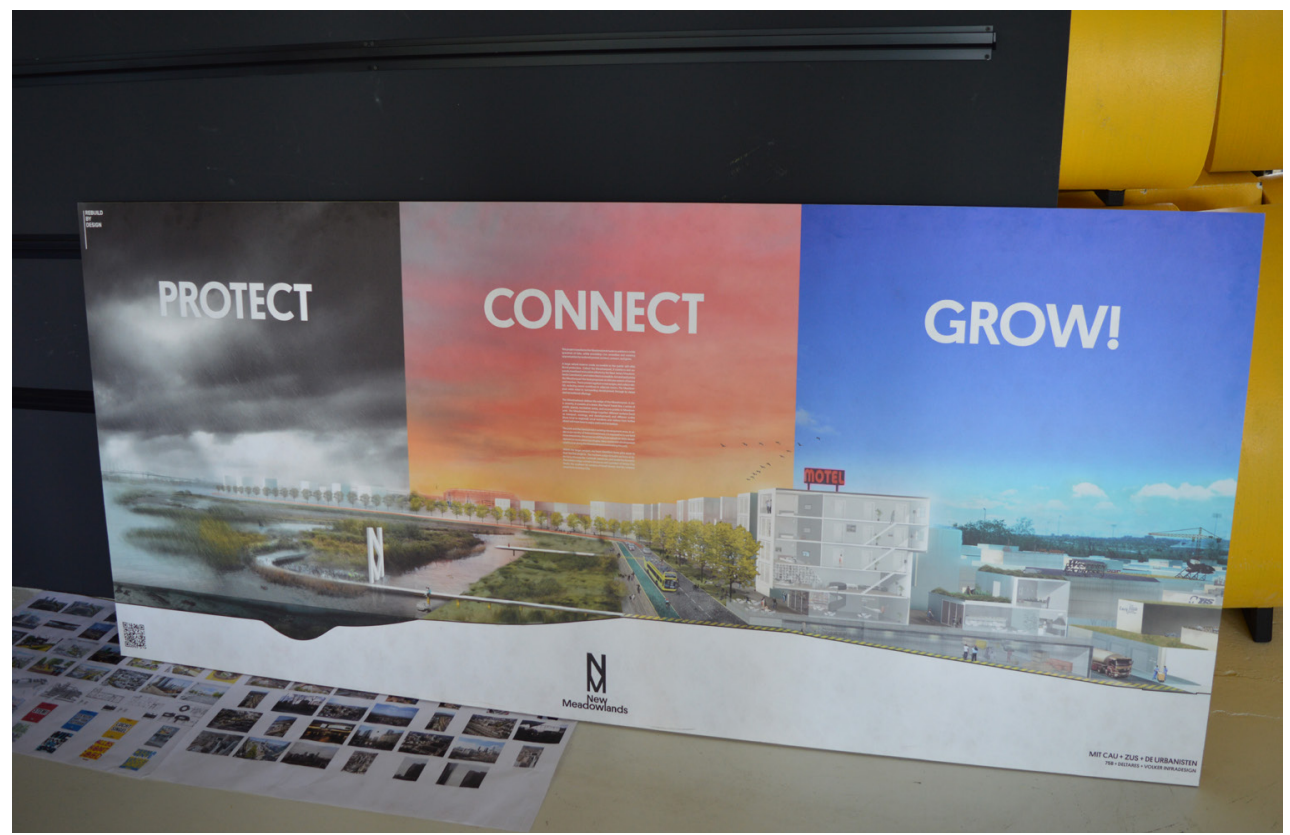

Figure 3.9. Interactivity: a board presentation with singular visual techniques (MIT-CAU et al. 2014). 


\section{$\nabla$}

\subsection{Discussion and conclusion}

This paper presents and demonstrates an analytic framework that enables a systematic visual analysis of landscape design representations. We conceptualize landscape designs as sign systems, to be interpreted by a range of different social actors and expressed through a variety of visual techniques and materials. Through this conceptualization, we are able to discuss design representations in terms of semantics, syntactics and pragmatics. The analytical framework that is based on these terms enables a visual content analysis and subsequent iconographical interpretation.

Our analytical framework is based on the assumption that dyadic and triadic semiotics complement each other. The writings of Eco demonstrate that such hybrid semiotics provide a comprehensive theory relevant to signification and communication, as well as an explanatory view on the logic of interpretation and possible over-interpretation. The issue of over-interpretation is especially relevant for an image-centred visual analysis such as presented in this paper. Overinterpretation occurs when, from a minimal relationship (between expression and content), the maximum possibility is deduced (Eco 1992). We therefore intentionally refrain from generalized conclusions that relate to the external meaning of the image, i.e. the meaning of the image in landscape planning and design practice. For example, it would not be valid to conclude that $\mathrm{x} \%$ of flood defence landscape designs are visualized using perspective $y$, technique $\mathrm{z}$, based on the data collection used in this study. Instead, our analysis and interpretation of the data focuses on the internal semantic and syntactic meaning and the iconographical context of the image.

Furthermore, when performing a content analysis one must be aware of its quantitative characteristics. As Rose (2012: 102) states, 'numbers do not translate easily into significance'. Not every occurrence is equally important. A large 
occurrence might even be less significant. For instance, considering that landscape planning and design are inherent spatial disciplines, the majority of the images are based on maps. Thus, the frequent occurrence of the code 'map' in the content analysis is in itself not surprising. Moreover, that which is not shown plays an equally important part in the significance of images. We address these 'invisible others' (Rose 2012) in our study during the syntactic analysis by considering a low co-occurrence of codes just as interesting as a high co-occurrence of codes.

As a semiotic entity, a landscape design representation contains categories and variables that always function in relation to each other. In our demonstration the co-occurrence analysis involves the code combinations of only two categories or variables at a time. A further, in-depth syntactical study is possible by looking at the co-occurrence of multiple variables and categories to take into account all the semiotic properties of the image. Nevertheless, the categories and variables defined in this paper do enable the systematic analysis of different design images. However, the list of codes that emerged during this study is not a general checklist for the interpretation of landscape design representations. Each landscape design project is different in terms of both content and expression. Consequently, the list of codes and semiotic descriptions that emerge from analysing another project will differ to a certain extent as well. To strengthen the scientific validity of these descriptions, the coding scheme could be subjected to a review by an expert panel of landscape practitioners to solidify, for instance, the definitions of more ambiguous, syntactic codes.

The descriptions of the syntactic relations and pragmatics are based on the iconographical interpretation of the researchers to demonstrate the methodological qualities of our framework. In practice, the final iconological layer might prove to play a decisive role in the interpretation of design representations. For instance, the novelty of digital visualization techniques can distract viewers from interpreting the content presented (Lewis et al. 2012). In this way, low content validity could be consciously compensated by the high interactivity of the medium. Similarly, 
showing the complexity of an urban microclimate system and claiming a high level of validity can consciously make the image more complex and unreadable. The iconographical interpretation should, therefore, be triangulated with its iconological meaning to comprehensively study the pragmatics of design representations. This can be done in a subsequent research phase through interviews with the producers and the intended audience of the images, or ideally by monitoring the design process. The analytical framework presented in this paper is, therefore, by no means a shortcut to the truth. This framework does offer a way of assessing the semiotic complexity of visual design representations as a starting point for a more in-depth, qualitative research strategy.

In conclusion, our analytical framework substantiates the perspective that design representations are more than just images to be looked at. They consist of several semiotic categories, i.e. mode, medium, knowledge and formulation which contain variables and codes that function through an interplay of validity, readability and interactivity. These terms, resulting from our operationalization of semiotic and iconographic theory for the disciplines of landscape planning and design, provide a new vocabulary with which to 'read', analyse, assess, discuss and create visualizations of landscape designs.

We demonstrated our framework by using two RbD projects as examples. This demonstration showed that qualitative data analysis software such as Atlas.TI enables the researcher to process a large amount of visual data in both an explorative and a systematic way. It also resulted in a list of semantic and syntactic codes with which the researcher and/or practitioner can create a semiotic topology within a visual database. This topology allows a researcher to identify semiotically complex images by filtering out the most and least occurring syntactics.

Furthermore, our demonstration shows that a qualitative interpretation of landscape design representations is possible through an iconographic lens. This lens enables a researcher to better understand, for instance, how textual anchorage helps explain certain visual choices. It also allows us to derive more contextual 
information from the image compared to a semantic analysis. In this way, it is possible to identify those signifying and communicating elements of an image that could lead to over- or under interpretation, which in turn could help to improve visual communication during participatory design processes.

Our conceptualization of landscape designs explains how planning and design practitioners, in making design representations, are also producing sign systems. From this perspective, the insights from our study can be used as visualization guidelines if we turn the process of sign interpretation into a process of conscious sign production. This can enable landscape planners and designers to consciously prevent possible misinterpretations of landscape designs by producing images that are more semiotically coherent. The ability to produce 'semiotically conscious' design representations would be a valuable skill for practitioners, especially considering the increasing complexity of participatory planning and design processes and the tendency for increased multifunctionality in future landscape design solutions.

\section{Z Acknowledgements}

The authors of this article would like to thank the anonymous reviewers for their positive and constructive feedback. The authors would also like to thank the Wageningen MSc students, MIT-CAU, and Interboro for the kind permission to use their image materials.

\section{Funding}

The research for this article was carried out in the context of the research programme 'Multifunctional Flood Defences', funded by the Stichting Technologie \& Wetenschap (STW), part of the Netherlands Organisation for Scientific Research (NWO). Additional 'in-kind' funding has been provided by knowledge institute Deltares. There is no conflict of interest. 



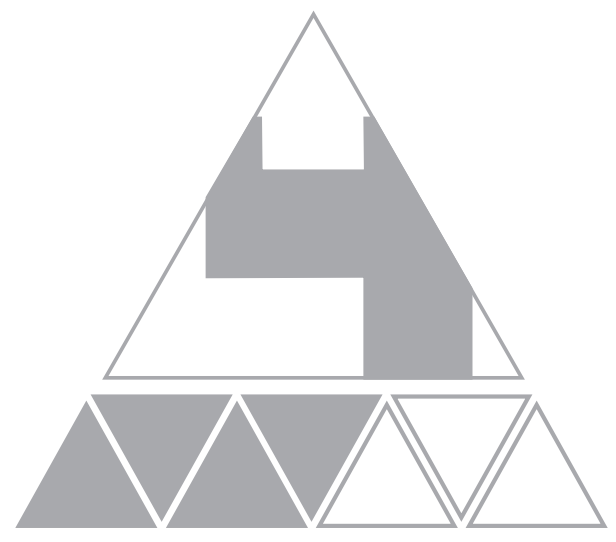


This chapter is published as:

Raaphorst, K. (2018). Knowing your audience: the contingency of landscape design interpretations. Journal of Urban Design, 23(5), 654-673.

Keywords

Design representation, landscape architecture, social semiotics, urban design, rebuild by design. 


\title{
Chapter 4
}

\section{KNOWING YOUR AUDIENCE:}

\author{
the contingency of landscape design interpretations
}

\section{$\pi$ \\ Abstract}

To address visual communication issues in landscape planning and design processes, an analytical framework that enables the study and possible anticipation of the interpretation of visual design representations is presented here. This framework consists of a hybrid theory of Peircean social semiotics and Laclaudian post-foundational discourse analysis (PDA). The semiotics of Peirce, through the concept of the interpretant, enable the conceptualization of the discourses that make up the socio-political contexts of design projects as so-called interpretive habits'. This framework is demonstrated by partly reconstructing the socio-political context of Rebuild by Design, a design competition organized in the wake of hurricane Sandy in New York. It is suggested through this demonstration that the sign systems and discursive networks that influence the interpretations of design images by different stakeholders can be partially uncovered during the design process itself. By recognizing these interpretive habits during specific phases of the design process, planners and designers could potentially better anticipate the productive and counter-productive interpretations of their design representations. 


\section{$x$}

\subsection{Introduction}

Landscapes and urban landscapes are the result of collective processes where multiple perspectives and perceptions engage in conflict and negotiation (Duineveld et al. 2017; Gailing \& Leibenath 2015; van der Stoep et al. 2017). The analysis of these discursive struggles is imperative to understanding how urban landscapes are constructed through participatory planning and design processes. The increasing interdisciplinarity of participatory planning and design projects (Thering \& Chanse 2011; van den Brink \& Bruns 2014) makes the socio-political context within which planning and design professionals operate more complex (de Jonge 2009). Consequentially, the outcomes of such participatory processes are not always interpreted and evaluated in the way planners and designers expect (Fleming 2016; Tobias et al. 2015).

In an attempt to improve understanding and reduce the unpredictability of interpretation the agency of plans and designs in landscape planning and design processes is becoming an increasingly important object of critical study (van Dijk 2011; Kallus 2016; Raaphorst et al. 2017). Furthermore, planning and design visualizations are 'being produced with new techniques and by new actors, within increasingly complex scopic regimes' (Swords \& Liu 2015: 1235). As a result, 'with images you do not know which fraction of the impression each viewer chooses or is coerced into seeing' (Dorling \& Hennig 2015: 1346). Several urban design scholars have engaged with (social) semiotic theory to study the role of such images in processes of place-making (Biddulph 2014), historical significance (van Assche et al. 2012), the power of planning imagery and cartography to influence planning decisions (Pojani \& Stead 2016), and even the creation of iconic projects in the building industry (Sternberg 1996).

However, it is still unclear how the divergent interpretations of planning and design representations by different stakeholders can be studied from a 
semiotic perspective and to what extent these interpretations can be anticipated. The research question central to this paper is therefore as follows: How are the interpretations of landscape design representations constructed by different stakeholders and to what extent can this be anticipated? To answer this question, two important 'research objects' are defined: the process of interpreting visual design representations and the socio-political contexts of participatory planning and design projects wherein these interpretations are constructed.

The process of interpretation, in Peircean semiotic theory, is conceptualized as the 'actualization of contextualized rules' (Gaspard 2016: 358). The Peircean concept of the 'interpretant' will be used to understand how these contextualized rules form 'interpretive habits' that guide interpretation (Peirce 1958). The socio-political context of planning and design processes is defined as a discursive sign system or network that ensures the 'control of both the production and interpretation of discursive acts' (Gaspard 2015, 554). Discursive networks are formed through semiotic relationships between signs that hold a particular significance for someone (Laclau \& Mouffe 2014; Marttila 2016).

The following section describes how Peircean semiotics and Laclaudian discourse theory can be combined to perform a 'social semiotic post-foundational discourse analysis' (SSPDA). To demonstrate the applicability and value of this method the SSPDA is applied to a participatory planning and design process. This process took place in the greater metropolitan area of New York as part of the regional design competition Rebuild by Design ( $\mathrm{RbD})$. This analysis demonstrates how the interpretation of design representations is influenced by discursive networks that constitute the socio-political context of participatory planning and design processes. The question of whether the interpretations of landscape design representations can be anticipated through such an analysis is discussed in the concluding section. 


\section{$\triangle$ \\ 4.2 Semiosis, the Interpretant, and Relational Ontologies}

Design representations can be defined as complex sign-systems that contain a large number of signs within themselves (Raaphorst et al. 2018). Different sign components, i.e. the visual characteristics and content of landscape design representations, but also the socio-political context in which they are used strongly influence the creation and interpretation of landscape designs (van Assche et al. 2012). The communication of design ideas through this creation and interpretation of landscape design representations can be defined as a triadic process of semiosis (Raaphorst et al. 2017). Eco (1976), following Peirce, defines triadic semiosis as an action or influence that involves three sign components: a representamen, its object and its interpretant. Together, these components enable a sign-function that facilitates the process of 'meaning making' through the interpretation of a representation of an object by someone. The cross-section of a dike, for example, represents the elevation profile of a proposed flood intervention, and can be interpreted by an engineer in terms of structural integrity.

As argued by Gaspard (2015, 2016), triadic Peircean semiotics show great potential for analysing discourse if 'discourse analysis' is defined as an inquiry into the production and interpretation mechanisms of meaning in contemporary society. This potential lies in the 'theory of Interpretants and the recognition of the role of interpretative habits regulating their adequate selection' (Gaspard 2015: 554). To clarify, the interpretant is not a person or interpreter. Rather it is a sign 'within the mind' that associates the object of one sign with the object of another sign (Eco 1976). The interpretant is a concept that enables us to think of meaning as something that is not fixed, but rather as an 'effect of signs dependent on a context' (Gaspard 2015: 553) and as a 'collateral experience' or 'previous acquaintance with what the sign denotes' (Peirce, as cited in Gaspard 2015: 557). Interpreting a sign in context means that one magnifies and neglects specific possible interpretants 
depending on the situation (Eco 1976). For instance, the interpretation of a crosssection by an engineer could lead from 'structural integrity' to flood safety norms, construction materials and building costs. A hydrologist might interpret that same image in terms of water flow capacity, sedimentation patterns and erosion problems. This selection of possible interpretants, tailored to specific contexts, is also called an interpretive habit. A sign is never interpreted without the habit necessary for its understanding, and it is the discursive context of the sign that determines how these habits are formed (Gaspard 2015).

In giving such a prominent role to the concept of the interpretant, the appropriate type of discourse analysis must be ontologically compatible with the Peircean definition of the sign and its context-driven interpretative logic. Furthermore, to render visible the unknown discursive context at work it is necessary to study local planning and design contexts in an open, exploratory way. The relational ontology of post-foundational discourse analysis (PDA) (Marttila 2016) enables such an exploratory study from a Peircean perspective. Post-foundational discourse theory underlines it is impossible to close any context along pre-defined structures. In postfoundational terms, 'discourse' can refer to 'any particular relational configuration of meaning-conveying objects (...) in which objects appear and are related to each other, and which constitutes the meaning (...) of these objects' (Marttila 2016: 155). This relational ontology makes clear that discourse can never be grounded on a stable foundation and does not signify a higher objectivity (Cederström \& Spicer 2013). Instead, the 'foundation' of discourse remains permanently contingent (Glynos \& Howard 2007). This contingency means that discourses revolve around affective forces that sustain or suppress them. A social semiotic perspective conceptualizes these affective forces in terms of the interpretant, i.e. a process of infinite semiosis by specific actors and institutions that forms the interpretive habits of those actors. The study of habits, as structures that depend on evolving sign-systems, allows one to anticipate the likeliness of particular interpretations 
to occur. Contingency thus embodies the realm of interpretations that could potentially occur rather than those that will occur with absolute certainty.

Besides their relational ontologies, Peircean social semiotics and postfoundational discourse theory are also compatible in their topology of semiotic relations and their explorative research perspectives. In post-foundational discourse theory the relations between semiotic objects are based on shared commonalities, or relations of equivalence and representation (Laclau \& Mouffe 2014). Recognizing the importance of this type of relations connects postfoundational discourse theory to Peircean triadic semiotics. Peirce distinguished specific functions of signification, i.e. relationships between signs and signcomponents, based on different kinds of equivalence and representation (Eco 1976; Jappy 2013). The relation of representation enables the articulation of the 'logic of commonality that binds discursive elements into a discourse' (Marttila 2016: 127). This relation is semiotic in nature, and, following Peirce's interpretant, can have an indexical, iconic, and symbolic sign-function (Jappy 2013). Indexical, if the signfunction is determined by causality. Iconic, if the sign-function is determined by similarity. Symbolic, if the sign-function is determined by conventional, cultural and emotional connotations. The contingency of interpretive habits entails the symbolic accumulation of indices (a non-linear sequence of events) and icons (qualities related to those events). Habits are formed when events, histories and experiences connect symbolically within a sign system or discursive network. From this perspective a reciprocal relationship is established: discourses determine the context for semiotic interactions, yet semiotic interactions and their typology also constitute the formation of discourses.

The explorative nature of PDA and Peircean social semiotics ensures that the prescriptive character of the ontological and epistemological omniscience of the expert' (Vannini 2007: 127) is rejected by embracing the interpretations of social actors in all their contradictions and complexity. Such an analytical strategy is necessary to do justice to the uniqueness of interpretations and to prevent 
mistaking a particular interpretation as the 'truth'. As such, researchers can never just encounter objective facts for them to be described. One can only arrive at a discursively constructed reality based on theoretically and culturally shaped descriptions (Torfing 2005). The objects of research are, therefore, entities that the researcher actively constructs, rather than entities that are 'ready to be identified and mapped' (Jørgensen \& Phillips 2002: 144). In short, 'doing' discourse analysis is not about describing a discourse, but about conceptualizing a discourse based on specified ontological premises. The analysis presented in this paper follows the relational ontology of post-foundational discourse analysis from a social semiotic perspective. The analytical framework for this social semiotic post-foundational discourse analysis (SSPDA) is presented in the following section.

\section{$\triangle$ \\ 4.3 Analytical Framework}

This section explains how four important concepts of post-foundational discourse theory, i.e. discursive identities, discursive relations, nodal points and discursive regimes (Marttila 2016), are operationalized for empirical analysis from a social semiotic perspective, and how these concepts can be studied using the technique of situational mapping.

Considered from the perspective of a relational ontology, signs or discursive elements, i.e. human and non-human meaning conveying objects, cannot possess inherent identities. The discursive identity, or the meaning of signs in relation to the discourse they are part of, varies according to the relations between elements. A map, a legislative document, a natural disaster, a mayor, or a design firm are all discursive elements that can be attributed identities of value, agency and function within a discourse depending on the perspective of the inquiry. To conceptualize discursive identities in this way means to identify their 'semiotic potential' (van Leeuwen 2005: 4) by constantly contextualizing all elements in relation to the perspectives relevant 
to the problem at hand. In doing so one does not describe what certain discursive elements mean, but what they could mean in relation to other elements.

If the discursive identities of discursive elements depend on their relations to other elements, these discursive relations between elements embody the typologies with which one element can be distinguished from another. These relational typologies of representation, i.e. icon, index, and symbol, and difference also lead to the identification of the elements that belong to the same discourse. Nodal points are formed through these relations of representation. Martilla, following the work of Laclau (Laclau \& Mouffe 2014), defines a nodal point as a sign that 'presents its particular features as the expression of something transcending its own particularity' (Marttila 2016: 49). A nodal point constitutes a sign that is 'bigger than itself' and thus signifies a larger concept or sub-configuration of signs. From a social semiotic perspective, nodal points are signs that represent the shared identity of different discursive elements, e.g. a design intervention that encompasses knowledges from various disciplines, and that function as a conceptual instrument to regard these elements as one entity.

Discourses are rationalized by social actors that reason from the perspective of specific social roles, e.g. the 'facilitator' or the 'project developer'. At the same time, social roles are 'discursive positionalities' rationalized by these discourses. For instance, the discourse of a specific stakeholder configuration in a participatory planning and design workshop influences the social roles of participants. Institutions such as the 'design firm' or 'state government' are mediated and rationalized by certain social roles and 'validate discourses by retaining their function as the epistemic foundation for institutional operations' (Marttila 2016: 137). For this concept PDA draws on Foucaultian analysis and considers this threeway reciprocal relationship between discourses, social roles and institutions to constitute a discursive regime. Such a regime can thus be defined as the situated enactment, validation and perpetuation of a discourse, or set of discourses, by specific institutions through actors that are assigned specific social roles. 


\section{Situational mapping of discursive networks}

Figure 4.1 illustrates where the concepts described in the section above are situated in a discursive network and how they relate to each other. The first step in analysing such a discursive network is the problematization of the social status of the research object. This entails defining particular problems in a specific sociopolitical context with the intention of deconstructing the 'taken-for-grantedness of the research object' and the 'presupposed self-evidential social meaningfulness' of previous attempts to define the problem (Marttila 2016: 140). This enables the open coding of discursive elements that, seemingly irrelevant to the research object, might form important nodal points and discourses underlying the problem at hand. The outcome of this analysis is a deconstruction of a socio-political context whereby these dominant discourses can be made explicit in the second step of a post-foundational discourse analysis. This second step involves the reconstruction of the research object by rendering visible the 'discourse(s) that was responsible for the historical formations and modifications of our research project in the focused

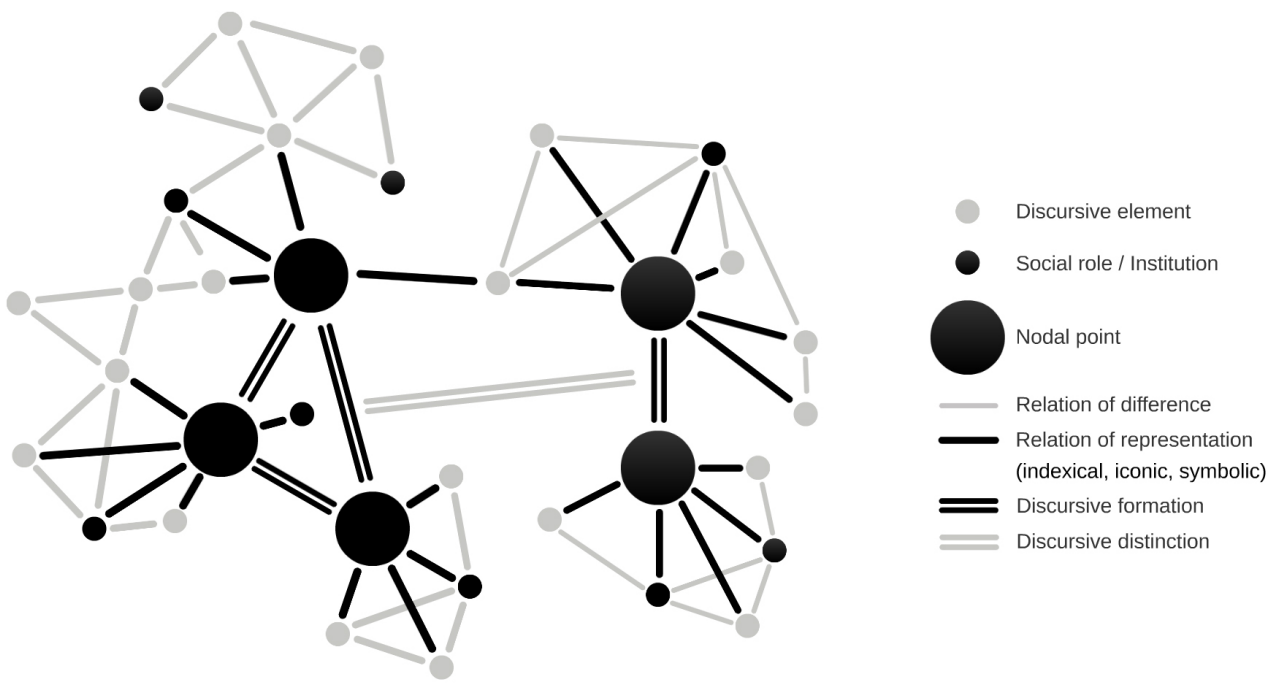

Figure 4.1. Example of a discursive network and its components (own figure, adapted from Marttila (2016) and Clarke (2005). 
spatiotemporal context' (Marttila 2016: 140). The emphasis on historical formation, i.e. the indexical sign-function of discursive elements and its linkages to other sign systems, provides insight into the formation of interpretive habits with which to analyse the contingency of interpretations.

Deconstructing and reconstructing discursive networks is done using the technique of situational mapping (Marttila 2016; Clarke 2003; Clarke 2005). Situational mapping is an analytical coding process that uses an incremental bottom-up strategy to identify the semiotic interactions in which the group of studied stakeholders partake. In the first step of deconstruction, the relevant discursive elements, or signs, are laid out descriptively in a rough situational map. In the second step of deconstruction the relations between these elements are identified in a relational map according to how they are described by the social actors who are part of the situation under study (first order hermeneutics).

In the first step of reconstruction the discourse analyst then reconstructs the relational map following a social semiotic relational ontology (second order hermeneutics). This distinction between first and second order hermeneutics is a key principle within post-foundational discourse analysis. Different elements are not necessarily part of the same discourse because social subjects think they have a similar meaning (first order); instead, they belong to the same discourse because they produce 'mutually consistent sets of relations' (Marttila 2016: 171) within networks of similarities (second order). Because it is the researcher that actively constructs these relations a constant critical reflection on methodological and analytical choices is needed to remain coherent within the theoretical foundations of discourse analysis (Torfing 2005).

In the second step of reconstruction nodal points are identified by looking at relations of equivalence and representation between elements, i.e. iconic, indexical and symbolic relations. These relations are then conceptualized: relations of representation group a number of nodal points into a discourse while relations of difference and contrariety between grouped nodal points distinguish one 
discourse from another. The final step of reconstruction consists of determining which discursive regimes are at work by identifying the institutions and social roles that produce and sustain the discourses previously identified. Social roles and institutions are identified by looking at which discursive identities are assigned to them by social actors.

\section{0 \\ 4.4 Rebuild by Design}

\section{Problematization}

The analytical framework of a social semiotic post-foundational discourse analysis (SSPDA), as it is described in the previous paragraphs of this paper, is applied to the socio-political context of the interdisciplinary participatory planning and design processes that were part of the Rebuild by Design competition. Rebuild by Design (RbD) started as a regional design competition in response to hurricane Sandy that struck the greater metropolitan area of New York, USA in 2012. Since then, $\mathrm{RbD}$ has developed into a regionally focused design approach for developing climate resilient areas all over the world (Ovink 2017). When the design competition started in 2013, one of its main premises was the recognition that a transdisciplinary participatory approach is needed to arrive at regionally applicable solutions that enjoy broad public and political support. Out of ten finalists, six design proposals were awarded funding for implementation. The project teams were assembled from design and engineering companies from across the globe with a substantial presence from the Netherlands. The RbD competition has therefore been characterized as a means to bring the process and expertise of the holistic 'Dutch' way of designing multifunctional flood defence solutions on a regional scale to the United States (Rebuild by Design 2015).

In 2016, two years after competition funds had been awarded, some of the design proposals were met with unforeseen public and/or political resistance 
(Fleming 2016) whilst others were making great strides towards implementation. Despite a carefully documented public outreach process, the lack of progress in the implementation of several projects suggests there are socio-political discourses unknown to the design teams that influenced the interpretation of the design proposals and that prevented design ideas from being implemented.

\section{Data}

Interviews were conducted with team members, stakeholders and advisors of several $\mathrm{RbD}$ proposals to study whether counter-productive interpretations of the design proposals could be traced back to the socio-political contexts of those projects. The body of knowledge used for the analysis represents the 'immediate social context', i.e. 15 transcribed interviews with designers, policy makers, engineers, hydrologists, academic advisors and local stakeholders involved in four RbD project teams: the 'New Meadowlands', 'Living with the Bay', 'Resist, Delay, Store, Discharge' and the 'Big U' proposals. 'General discourses' i.e. written reports of both the design processes and the stakeholder meetings that took place during those processes, were used to contextualize the interviews. To demonstrate the SSPDA method the data consists of excerpts from interviews with a member of the environmental community, a project developer, an ecologist, an organizing member of a Citizens Advisory Group (CAG), an urban designer, and two landscape architects. Other interviews, observations and literature have been used to contextualize the demonstrated example in the wider perspective of $\mathrm{RbD}$ as a design competition.

\section{Interviewing technique}

While collecting data for a social semiotic analysis the context of the research object is mapped in an explorative way to trace the formation of interpretive habits. This was done by using an open-ended semi-structured interview strategy and by refraining from asking any closed questions. During these interviews, a 'probing' 
strategy has been used to attain socio-semiotic responses, i.e. detailed descriptions of how the respondents experienced the use of visual semiotic resources (Vannini 2007). These probes consisted of design visualizations as stimulus objects (Törönen 2002). Törönen (2002) distinguishes three ways in which stimulus objects might function semiotically during an interview: as a clue, as a microcosm and as a provoker. These three functions relate to the Peircean distinction of index, icon, and symbol, respectively. As an index, the stimulus object is relational: what role did a certain design visualization play during the design process; was it an important product that helped to advance the process or did it cause a stagnation? As an icon, the stimulus object stands for a larger whole. It is representational: to what extent does this image reflect your expertise, do you recognize your input in these designs, in what way is this expressed visually? Finally, as a symbol, the stimulus object can be used to elicit strong personal reactions. What does this image mean to you personally? Are you content with the design or displeased by it. If so, why?

\section{0 \\ 4.5 Co-constructing Rebuild by Design}

In a step of first order hermeneutics the section below describes the historical context of the Meadowlands area and its conservation laws as it is recalled by some of the project participants of the 'New Meadowlands' project proposal (MIT-CAU et al. 2014). In the successive section the identification of discursive elements and nodal points that constitute the most dominant discourses that are relevant to the interpretation of the design representations is described in a step of second order hermeneutics. This demonstrates how significant discourses that surround the $\mathrm{RbD}$ competition can be (re)constructed. In describing the process of discourse analysis the same incremental bottom-up strategy is applied as during the analysis to illustrate that participants' descriptions (first order hermeneutics) contain signs or discursive elements. These elements, depicted in bold, form sign 
systems and, through a typology of semiotic relations, are used to identify nodal points that represent the components that make up a larger discourse (second order hermeneutics).

The New Jersey Meadowlands is a 30.4 square-mile $\left(79 \mathrm{~km}^{2}\right)$ area that encompasses two counties, fourteen municipalities and a large preserved wetland area. An historical precedent regarding large-scale development in the Meadowlands area has played a formative role in the attitude of the environmental community towards spatial development projects. This history has been described by an ecologist who is an assistant professor at a local university in the State of New Jersey and advisor to the project team. She recalls the story as follows:

\section{Ecologist local university:}

About 20 years ago, there was a big proposal for development, for a shopping mall. (...) and it was an international developer (...) They wanted to fill, about 300 acres of wetlands. (...) It would have been the largest wetland fill east of the Mississippi river since the United States passed the clean water act. (...) And the environmental community just stood up and said: no more. And they were fighting against the New Jersey Meadowlands Commission, they (the meadowlands commission - KR) were a regional planning agency (...) both those entities supported the project. The developer (...) had strong connections in Washington. So there were a lot of very powerful people who wanted this to go forward, and after many years of fighting this through the courts (...) they (the environmental community - KR) actually won the fight. And that land is preserved now in perpetuity.

It took the environmental community a lot of effort to fight against the project. Their efforts resulted in the solidification of the clean water act, which permanently preserves the wetlands for their natural functions and is considered to be their crown achievement. The legislative power of the clean water act and other 
environmental laws is recognized by the environmental community and developers alike. A local project developer, key stakeholder in the New Meadowlands design project team, explains:

Project developer:

Our environmental regulations preclude flood control today, pretty much. (...) Petition wildlife, clean water act, national resources (...) let's assume you have a blank cheque (...) you can't get these permits.

Semiotic relations, nodal points, and discursive regimes

The first nodal point that can be identified is the 'clean water act' (Figure 4.2). This sign symbolizes the history of resistance against urban development in the wetland areas by the environmental community and against the shopping mall development proposal in particular. It is also iconic of the other environmental laws at work, such as petition wildlife, that prevent any development in the wetlands. At the same time it serves as an index for the courts that ruled in favour of the law. However, that legislative power alone is not considered to be enough to preserve the wetlands. The chairman of a local environmental protection agency and key stakeholder in the New Meadowlands project emphasizes the need for the environmental community to safeguard the implementation of environmental laws:

Environmental protectionist:

In the US we have laws that govern these kinds of things, that are not well enforced, unfortunately. If they were well enforced there would be no need for me. (...) The clean water act (...) said all our waterways will be fishable and swimmable by 1985. Has not happened. And every time you turn around there is a new threat to the quality of the water, the availability of the water. 


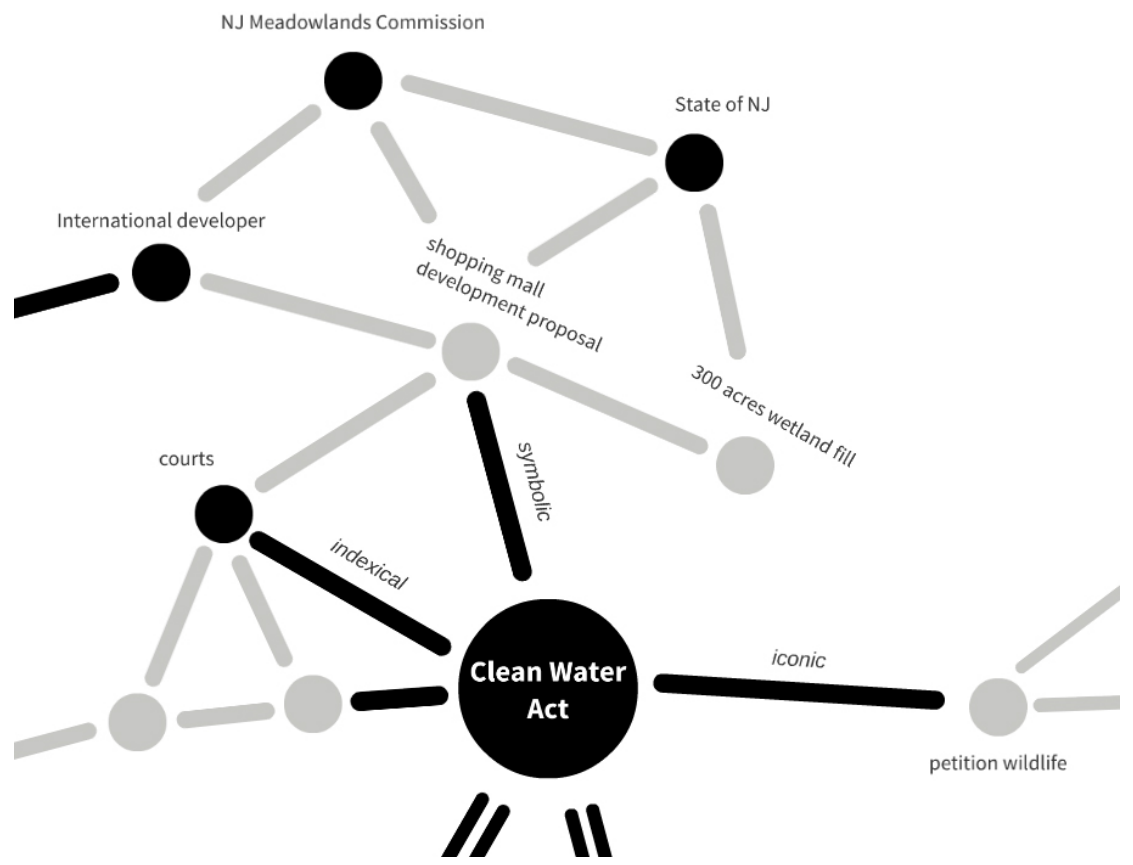

Figure 4.2. Formation of the 'clean water act' nodal point (own figure).

The political power-play surrounding the wetland fill project, i.e. the development being supported by the government and the improper enforcement of environmental laws since then, connects the clean water act to another nodal point: 'failing government'. For example, according to the interviewee, this nodal point is an index for the type of politicians involved in State governance and their career interests:

Environmental protectionist:

Politicians are the last that need to be making these decisions, (...) their attention span is as long as their term of office. They want to make sure that nothing bad goes down on their watch.

This nodal point is also iconic for the recent changes in State administration and the merging of conflicting interests: 
Environmental protectionist:

The New Jersey DEP (department of environmental protection - KR) has an assisting commissioner for economic development embedded in the department, and that's wrong. (...) the environmental protection department is supposed to be the ones that stand there and hold the line (...) but now they are not allowed to deny permits if the assistant commissioner says that New Jersey needs this economic boost, so all bets are off. (...) I knew right away when they announced that the grant (design competition funding - KR) would go to the DEP that we're in trouble.

This resistance of the environmental community to any policy coming from the state government is an index for a number of recent activities undertaken by the current governor's administration that have strong symbolic connotations of hostility for that environmental community:

Ecologist local university:

There was a claim, a 9 billion dollar claim that had gone through the courts for years, that the state of New Jersey was suing (anonymized international oil and gas company - KR), and the judge was ready to roll on a claim (...) because they have been found guilty (...) and the state settled for 215 million, against a 9 billion dollar claim, (...) and this was in the middle of (current governor's - KR) presidential run, so that just added fuel to the fire. They commissioned a study that they want to privatize a significant portion of Liberty State Park, where they have private concessions, so that's very controversial, they disbanded their regional planning entity, the Meadowlands Commission.

The 'failing government' nodal point refers to that perceived hostility towards the environmental community in a broader sense, and connects to the nodal point 'clean water act' indexically as an interpretive habit due to the lack of implementation 
of that law in practice. Together, the historical formation of these nodal points form the discourse of 'environmental preservation', depicted in Figure 4.3. This discourse and its habits constitute the contingency of how the designs presented during the planning and design process of the 'New Meadowlands' Rebuild by Design project can be interpreted. For example, a map (Figure 4.4) depicting a flood barrier in the preserved wetland triggered the following interpretation from the perspective of the environmental protectionist:

\section{Environmental protectionist:}

They showed us a map of where they wanted to place these 30 foot high barriers, where they were looking to go right through conservation land. (...) we worked really hard to protect what's left, one of the reasons being that that's (conservation land - KR) our first line of defence against these kind of activities, these kind of disasters.

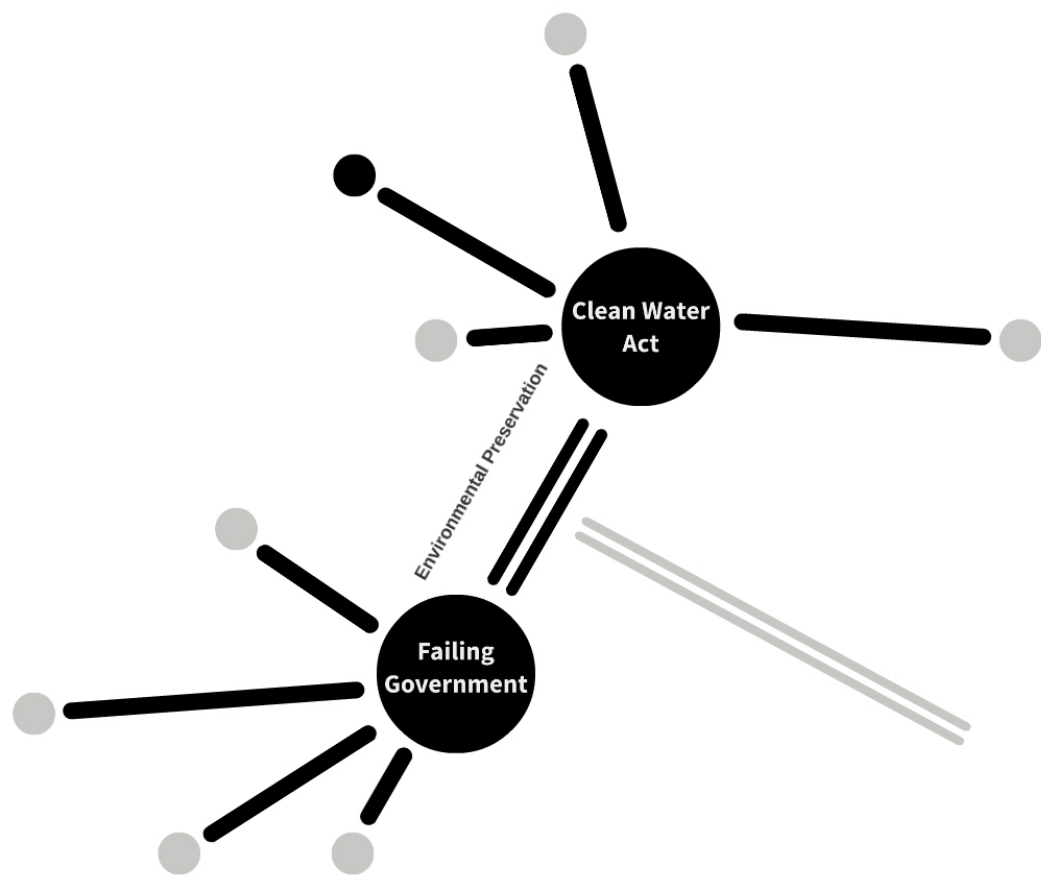

Figure 4.3. Formation of 'Environmental Preservation' discourse (own figure). 


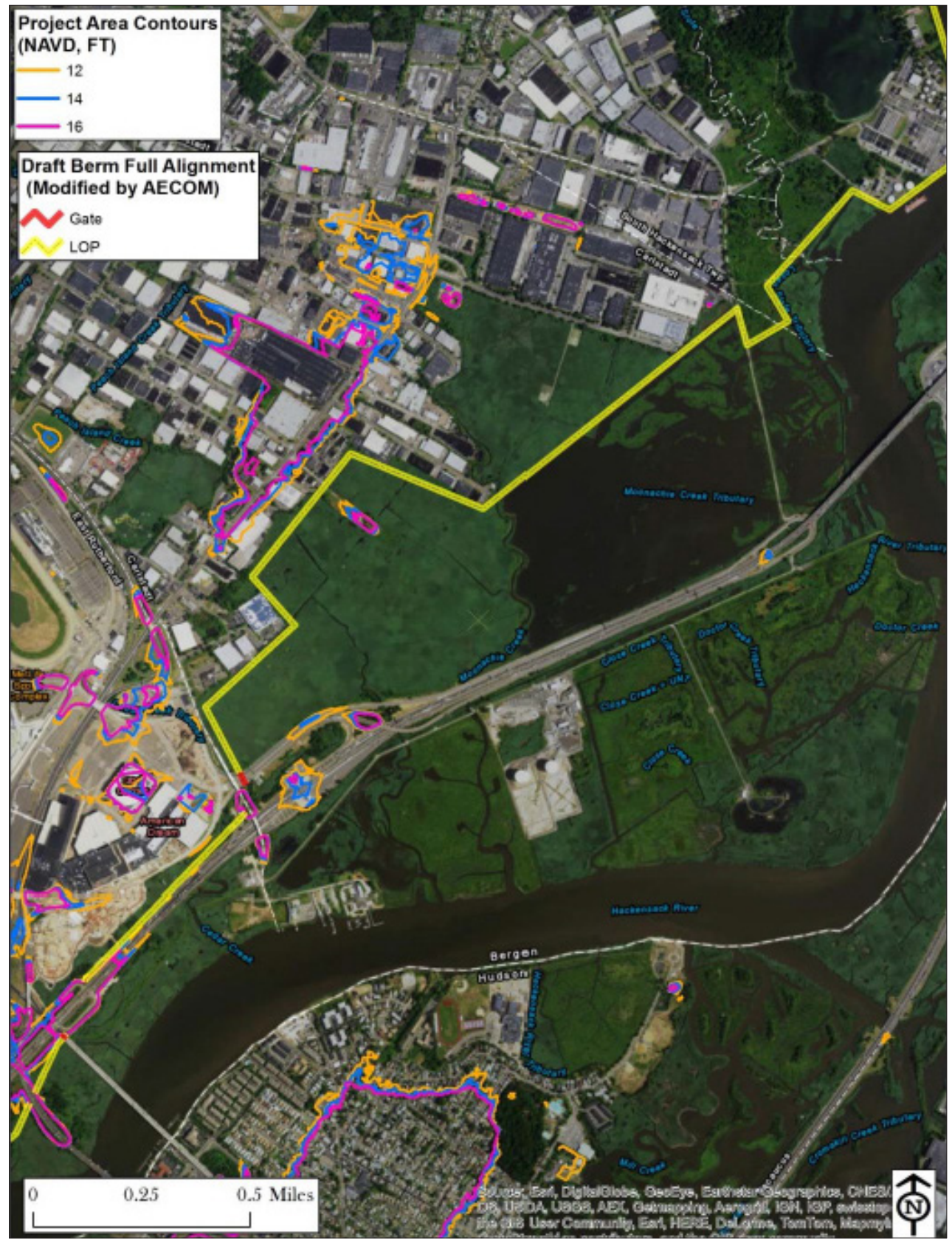

Figure 4.4. Example of a map that depicts the 'Preliminary Flood Protection Alignments' in a part of the Meadowlands. The proposed location of a berm through the conservation land is depicted in yellow. (New Jersey Department of Environmental Protection). 
The discursive regime of 'environmental preservation' (Figure 4.3), linked to the map in Figure 4.4, is perpetuated by the environmental community in which one person identifies his own social role and institution, as well as the agency of actors attached to his organization:

\section{Environmental Protectionist:}

Besides being the river keeper, I' $m$ the chairman of the (anonymized) Conservation Trust, which owns several very large wetlands areas for conservation purposes. Nothing can ever be built there, it can only be managed as a natural area. They had the line for the dike drawn right through the property, without ever consulting me, or my board, or my attorney. (...) I said: if you try I will tie you up in court, and it will be just long enough for them to forget that they wanted to do it.

The link with the judicial system and the resulting power to legally slow down development projects is iconic for the key measures the environmental community can take. Moreover, the social role of the environmental protectionist is not only an icon for being the chairman of an organization, it is also a symbol of the reason why this organization exists and how it is funded:

\section{Environmental Protectionist:}

We have our foundational grants, philanthropic foundations, that fund us (...) And then we have our donor base (...) People have made donations, some of them in the thousands of dollars, some of them is in the 10 to 15 dollars. You know, it's public support. The public believes in what we do.

The analysis presented above illustrates how the discursive regime of environmental preservation, its social roles and institutions, nodal points and sign systems can be reconstructed using a social semiotic post-foundational discourse analysis. Environmental preservation, along with other significant discourses, 
forms a discursive network that surround(ed) the Rebuild by Design participation processes of which the following section provides a more abstract overview.

\section{Discursive networks}

Movements of self-organization through community engagement, such as the river keeper organization, form a nodal point habitually as an index for the need to protect interests that are otherwise unprotected. The nodal point of 'community engagement' connects to another nodal point, i.e. 'mandate', through symbolic necessity: the nature of self-organization as the result of various NIMBY sentiments ensures the mandate of this type of community engagement. As one of the leading landscape architects from the BIG-U team explains:

Landscape architect from BIG-U team:

These community organizations are so active because that is the only way of preventing they'll be disadvantaged. That is what a lot of these processes (Rebuild by Design community outreach - KR) are about as well: to make sure that especially corporations and wealthy white people don't get the chance of displacing the more vulnerable communities.

Social vulnerability constitutes another important nodal point, as it ties into the aforementioned issue of governmental distrust:

Landscape architect local university

Social segregation within New Jersey is according elevation: higher land is safe and desirable, lower land is unsafe. So you have low income groups on unsafe land. (...) these folks have a massive distrust in the government and have the experience that the government does not care for them, but for the big investor. 
These nodal points of community engagement, madate, and NIMBY form a discourse of 'grassroots movements' (Figure 4.5).

This sense of community connects once again to another dominant discourse: that of 'local identity'. The urban developments proposed by the design teams, visualized in Photoshop images (Figure 4.6), are iconic for a degree of building density and gentrification that seems to contradict the symbolic idea that local inhabitants have of the area. A project developer, key stakeholder in the New Meadowlands project, explains:

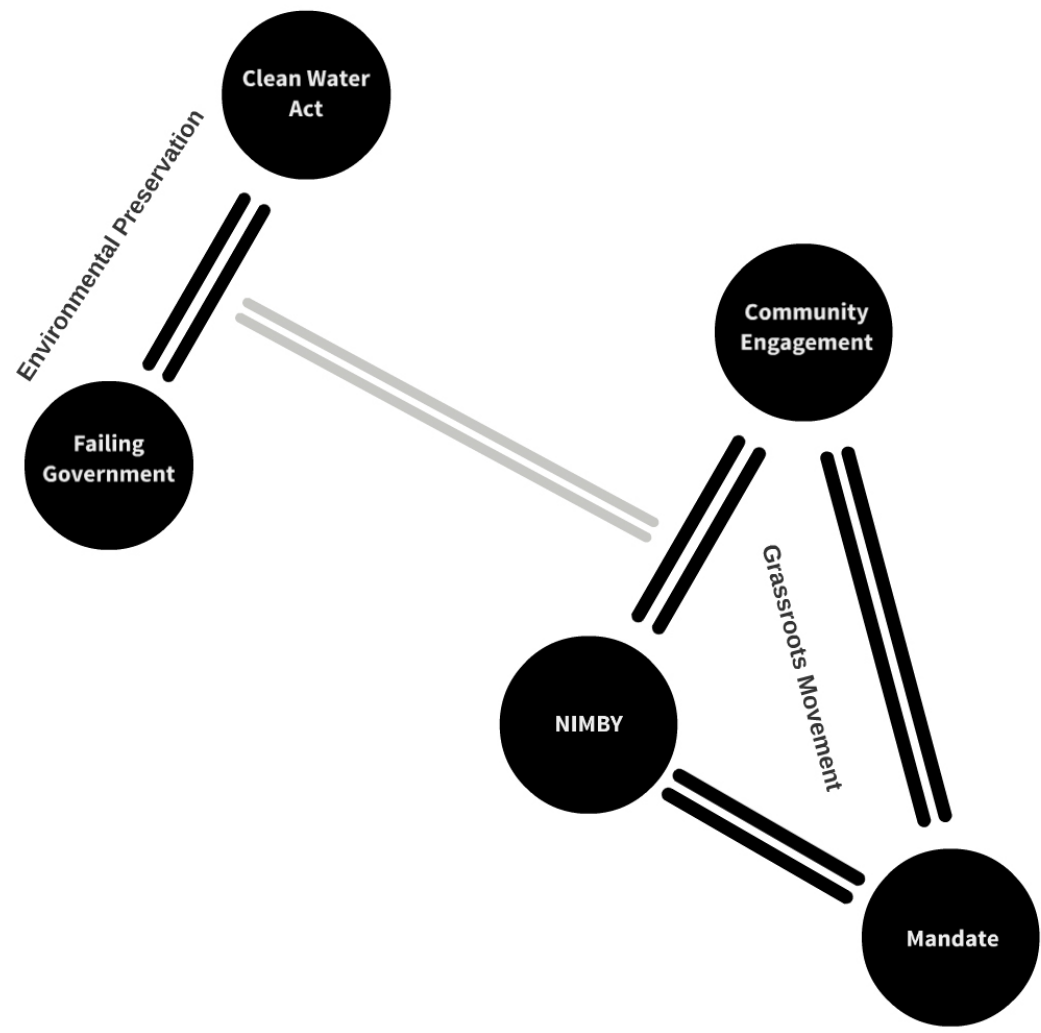

Figure 4.5. Discursive formation of 'environmental preservation' and 'grassroots movement' (own figure). 
Project developer:

People in New Jersey are afraid of density, even though it's the densest populated state in the country, they saw apartment buildings, and when they see apartments they think: transients, young professionals, people that may move from NYC.

Despite the seemingly apparent 'density' of the Meadowlands area this nodal point is symbolically connected to another nodal point, i.e. 'small communities'. The Photoshop image showing new urban development along the proposed berm (Figure 4.6) triggered a counter-productive interpretation amongst several stakeholders. The environmental protectionist, key stakeholder in the New Meadowlands proposal explains:

Environmental protectionist:

They had pictures of apartment buildings (...) all these high rises, and right now along that property line there there's probably all individual homes (...) that gives it that small town flavour that people want. People that live in the Meadowlands live there because they don't like New York city.

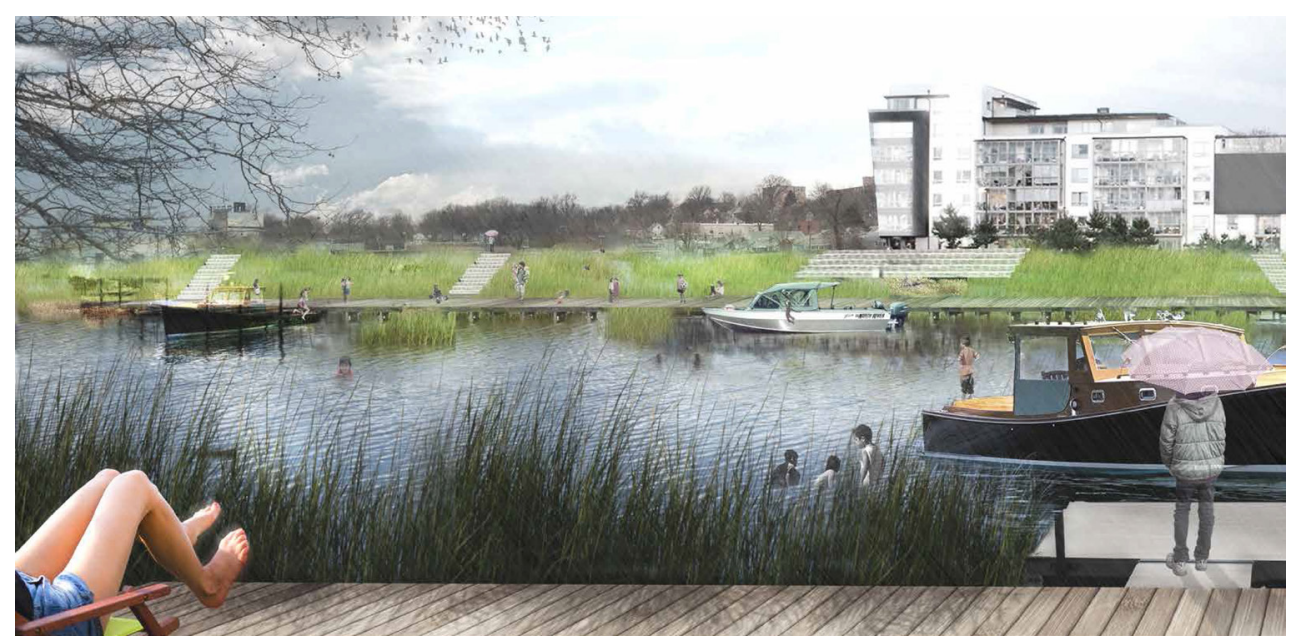

Figure 4.6. Example of Photoshop rendering of urban developments along the Hackensack river in the Meadowlands (MIT-CAU et al., 2014). 
To the local stakeholders, the Photoshop images showing new urban development symbolized an image of wealth and the political institutions behind that wealth that contradict their idea of small communities:

Landscape architect local university:

People know this kind of graphic language from rich investors and rich people.

(...) New York Times magazine, the first couple of pages are all about these investments and big fancy homes, and that's the same graphics.

This type of graphic language is inextricably linked to the nature of design competitions. Rebuild by Design, as a competitive jury-led and time-pressured process that is focused on simultaneously achieving innovative flood defence solutions, transdisciplinarity, public participation, and political support, placed the design teams in a complex web of partly irreconcilable sign systems that gave rise to conflicting interpretants. For instance, the position of the design teams in relation to the implementation of their own projects in the long term was uncertain:

Lead urban designer OMA team:

The amount expected from the teams in the competition was unusually high (...) huge investments from the teams (...) it didn't actually mean you won funding for the project, you won funding for the state to implement the projects.

This overturning of the design process to the State exposed the differences in politics and culture between the implementing agencies and the design teams:

Lead organizer Citizens Advisory Group Hoboken:

Yes there were interdisciplinary design experts involved, and yes there was a wide section of the public, but as the money (...) gets turned over to the public agencies, because its government money, it goes from being a community and 
participatory NGO driven process to a bureaucratic and public procurement process. (...) A government entity as opposed to an NGO (...) their interests and their value system, and their matrices quite honestly are different.

The design teams were presented with the challenge of facilitating public outreach sessions whilst at the same time catering to the expectations of potential implementing agencies. The visual representations that were attuned to the interpretants of project developers and implementing agencies had an adverse effect on the interpretations of those same images by local stakeholders. The interpretive habits of local stakeholders, i.e. the sign-systems that actively guide their interpretations, differs substantially from those of the implementing agencies. Having the state government as their client made the design teams prioritize communications attuned to a specific audience, despite the disruptive power that one local stakeholder and his or her lawyer might have on the implementation of their projects.

The examples above illustrate that mapping a discursive network following a relational ontology from a social semiotic perspective results in myriad connections between discourses and nodal points. Nodal points, such as 'community engagement', or 'failing government' can also belong to several discourses at the same time, acting as points of convergence within a network. In the following section it is discussed how one can arrive at a selection of the most significant interpretive habits and discursive regimes, and how one can determine the limit of the context that needs to be mapped. 


\section{$\nabla$ \\ 4.6 Discussion}

The excerpts described above only reproduce parts of the social-political context of the $\mathrm{RbD}$ competition. In practice, it is imperative to come to a selection of the most significant discourses at play to determine with which interpretive habits landscape planners and designers need to engage. This selection is based on the semiotic relationships between elements and nodal points, as well as on the proponents of the relevant discursive regimes, i.e. social roles and institutions. The significance of semiotic relationships is primarily dependent on their semiotic topology, i.e. whether these relationships are symbolic, iconic or indexical. For instance, a chain of indexical relationships constitutes a strong causality of events, e.g. the history of urban developments in the New Meadowlands wetlands, and thus has a strong ontological significance for habit formation. Chains of symbolic relationships, e.g. the perspectives and values of the environmental community and the project developer as a result of the historical developments in the area, map the range of contingent interpretations of the New Meadowlands design by those stakeholders.

For Peirce, 'the identity of a habit depends on how it might lead us to act, not merely under such circumstances as are likely to arise, but under such as might possibly occur, no matter how improbable they may be' (Peirce 1992: 131). This means that the interpretive habits that belong to social roles and institutions, as proponents of discursive regimes, determine how design representations are interpreted and acted upon. By extension, these habits constitute the context of the audience with which the designers need to engage. Determining the semiotic hierarchy of discourses and their proponents is heavily dependent on the semiotic typologies attributed by the researcher. A comprehensive and conscious approach to the first order hermeneutic coding round of the analysis is therefore crucial 
for a consistent reconstruction of interpretive habits during the second order hermeneutic coding round.

The premise of the relational ontology and explorative approach of SSPDA advocates developing the ability to uncover latent discourses that would otherwise be overlooked. It does so by detailing a discursive network that allows the tracing of the interconnectedness of all material and non-material signs, nodal points and discourses. In turn, this could make it possible to anticipate design interpretations not only by looking for dominant power structures, but also by identifying other, latent semiotic relations whose significance has not immediately been acknowledged yet which can shape the contingent effects of interpretive habits.

The incremental approach to the explorative mapping of discursive elements and relations to arrive at interpretive habits does raise questions of interpretive drift' (Eco 1990). If, as Derrida claims, infinite semiosis is an endless deferral of meaning (Baugh 1997) one is confronted with the challenge of determining the 'limit of context'. The discourse analyst needs to know when saturation is achieved in mapping the context. This can be problematic if every sign is potentially connected to another sign, as it is presupposed by the relational ontology of postfoundational discourse analysis. Eco invoked the Peircean definition of the sign to explain that unlimited semiosis does have a sense of direction, as opposed to an interpretive 'free for all'. Peirce stated that 'a sign is something by knowing which we know something more' (Eco 1990: 28) ‘Knowing more' implies that the deferred interpretation enriches the meaning of a sign within a specific context. During this process of 'enrichment' meaning accumulates in relation to different sign systems as specific interpretants are highlighted in conjecture with a given 'universe of discourse' (Eco 1990). This means that by defining a problem-driven universe of discourse to interpret the research object one also determines the openness of the analysis. This raises the crucial question whether a SSPDA can still be performed to uncover discourses without a-priori excluding them. 
The answer lies in the combination of the problem-driven contextualization with a theory-driven contextualization of the research object (Marttila 2016). The limit of context is thus constructed during the first order hermeneutic analysis of relational interpretations by the interviewees themselves. The construction of discourses within that context is done during the second order hermeneutic analysis. In this way the limit of context emerges from the open-ended collection of empirical data, whilst the semiotic interrelatedness of discourses within that context, as the historical formation of their interpretive habits, is incrementally constructed by the researcher.

Is it possible, then, to arrive at the anticipation of stakeholders' interpretive habits provided that a SSPDA enables the (re)construction of a relational sociopolitical context? An interpretive habit requires, according to Eco, 'a community as an intersubjective guarantee of a non-intuitive, non-naively realistic, but rather conjectural, notion of truth' (Eco 1990: 39). The contingent and conjectural nature of interpretive habits implies that these specific interpretations are, to a certain degree, shared between stakeholder groups. At the same time, the notion of contingency also implies that the 'collateral experience' of stakeholders continuously changes. Contexts, and therefore sign systems, evolve or come into play during the different stages of a planning and design process. Moreover, the interpretations of different design iterations 'enrich' the habits of stakeholders as one visualization follows another. The mapping of discourses and the anticipating of interpretations is thus an ongoing activity tailored to each design stage and stakeholder configuration, and thus to changing local political and cultural sensitivities.

The contingency of interpretive habits arises from the historical formation of discursive networks coupled with the discursive agency of social roles and institutions within discursive regimes. However, to anticipate which range of contingent interpretations is 'productive' for a design process, one first has to determine the range of interpretations that is theoretically 'counterproductive' (Eco 1990, 1992). The relations of difference and contrariety help to anticipate these 
'counterproductive' interpretations by identifying nodal points and discourses that cannot be reconciled with the content or expression of a design representation. For example, the Photoshop representation showing urban development (Figure 4.6) could have had the intention of showing the increased level of flood protection of the hinterlands as the result of a proposed berm. However, this image contradicts the discourse of local identity with its nodal points of small communities, gentrification, density and failing government. Taking this incompatibility into account the designer could look for other ways to visualize the possibility of urban development due to increased flood protection, or rethink the ways to express flood safety in general.

\section{$\nabla$ \\ 4.7 Conclusion}

The relational conceptualization of discursive formations, as described in this paper, demonstrates how specific discourses within the socio-political context of the $\mathrm{RbD}$ processes influenced the interpretation of the representations of the $\mathrm{RbD}$ process outcomes. As discourses express themselves through signs, or a collection of signs, landscape planners and designers may be able to identify discursive networks during the different phases of participatory planning and design processes. An SSPDA enables the recognition of these networks, which then could enable the landscape planner or designer to anticipate which discourses influence the interpretation of the ideas that are embedded in a design representation. For example, when a stakeholder informs a designer that the proposed design solution is impossible due to a violation of environmental laws one should not interpret this statement as a mere notification of legislative facts. One should interpret this statement as a sign connected to other signs that make up a discourse that influences an interpretation that could prevent a design from being implemented. When confronted with a sign, or nodal point, such as the Clean Water Act the designer 
could then proceed by asking why it is impossible to build in that area, how powerful these environmental laws are, how these laws came into being, and which actors actively enforce them. These questions could uncover a discursive network that helps to anticipate the design's interpretation in consecutive design phases.

By adapting a more post-foundational and social semiotic perspective, planners and designers can become more sensitive to how discourses inform what people think and do, what kind of stakeholders are involved and how they present themselves in interaction with others. The notion of contingency helps planners and designers to think in possibilities instead of certainties and enables the identification of signs with strong 'semiotic potential', such as the clean water act or the high rise buildings depicted in an artist's rendering. Moreover, such a perspective helps to understand how habits are formed, and consequentially, how they can be influenced through particular visualization choices that have the discursive power to do so.

Ideally, plans and designs resulting from participatory processes reflect the input and imaginations of local communities, the knowledge of disciplinary experts, and the wishes of a project commissioner. Attuning design representations to specific socio-semiotic discourses is about depicting specific content in ways that resonate with the images' intended audiences. In turn, more consciously attuned visualizations could help attain public and political support for implementing a design based on its content rather than on the way that content is visually expressed. 


\section{要 \\ Acknowledgements}

The research for this paper was carried out in the context of the research programme 'Multifunctional Flood Defences', funded by the Stichting Technologie \& Wetenschap (STW), part of the Netherlands Organisation for Scientific Research (NWO). Additional 'in-kind' funding has been provided by knowledge institute Deltares.

The author would like to thank the anonymous reviewer(s) of the Journal of Urban Design for their constructive and insightful commentary, as well as the WUR colleagues that provided helpful comments on earlier iterations of this paper. Furthermore, the design offices of MIT-CAU, ZUS and Urbanisten and the New Jersey Department of Environmental Protection are also thanked for the kind permission to use their image materials in this paper. 



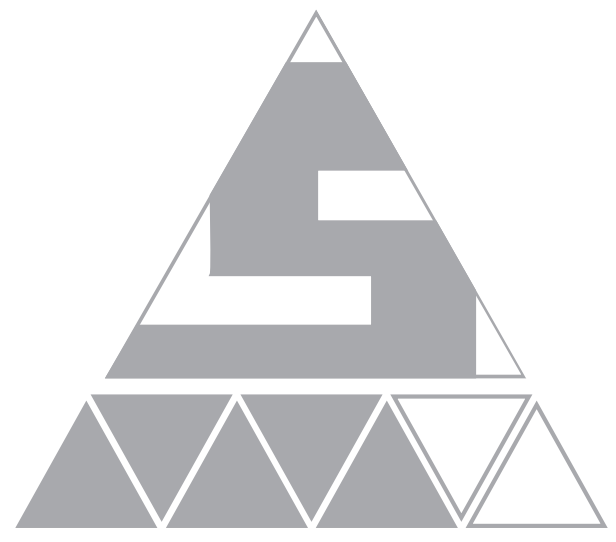


This chapter is submitted as:

Raaphorst, K., van der Knaap, W., Roeleveld, G., and van den Brink, A. Visualization, Participation and Rhetoric: The Discursive Power of Landscape Design Representations in Participatory Processes. Journal of Landscape Architecture (in review).

Keywords:

Design visualization, visual discourse analysis, participatory design, visual rhetoric, rebuild by design 


\title{
Chapter 5
}

\section{VISUALIZATION, PARTICIPATION AND RHETORIC:}

the discursive power of landscape design representations in participatory processes

\begin{abstract}
$\pi$
Abstract

Visual representations of landscape designs are used to facilitate communication and knowledge exchange during participatory planning and design processes. The production of design representations during such processes is considered to be a discursive act: actors and institutions construct specific knowledge with a certain authority and credibility during a design process through the use of visual expression. In this paper we aim to understand landscape design representations as discursive materializations of power and knowledge by studying the social relations within which the production of representations is embedded and how these relations manifest themselves in the communicative qualities of design representations. An analytical framework for conducting a visual discourse analysis is presented and applied to the transdisciplinary design competition of Rebuild by Design. The analysis uncovers interdependencies between three components of visual discourse: the arrangement of the participatory process, the interactivity of the media that are used, and the visual rhetoric embedded within the composition and style of the image. A conscious use of these discursive components could help prevent miscommunication, manage participant expectations, and increase the validity of participatory design process outcomes.
\end{abstract}




\section{$x$}

\subsection{Introduction}

Within the context of (re)designing flood defence landscapes planners and designers are increasingly required to arrive at multifunctional landscape solutions (van Veelen et al. 2015). This multifunctionality entails combining existing and new spatial functions with the participation of a wide range of experts and stakeholders. Landscape planners and designers use various kinds of visual representations to facilitate the communication of design ideas among project participants (Gill et al. 2013; Kallus 2016). For example, the planning and designing of multifunctional flood defence (MFFD) landscapes involves, among many things, the use of sketches, maps, cross-sections, photomontages, artist impressions, and 3D models of diverging visual complexity (Raaphorst 2017).

Design representations are not considered as neutral communication devices (Barnaud et al. 2013). For example, the act of drawing is seen by Dee (2016) 'less as a technique, and more forcefully as an experimental method' (Dee 2016: 52). Designers and planners facilitate the exchange of knowledge among scientific experts, stakeholders and local inhabitants through visualization by either letting participants draw or by mediation of the designer (Kempenaar et al. 2016). As intermediaries, landscape architects 'provoke situations of exchange and dialogue between a place and a public' (Keravel 2010: 70) wherein different degrees of participation reflect the interactivity of an image with its audience. For participants the ability to draw and the level of access to the production of visualizations define the influence of those participants on the outcomes of planning and design projects.

The conscious and unconscious use of increasingly accessible digital visualization technologies by spatial designers has become an important topic of scholarly debate (Downes \& Lange 2015; Kingery-Page \& Hahn 2012). For example, it is argued that digital technologies enable designers to think on higher levels of complexity and achieve different solutions in terms of systems, form, 
and materiality (Walliss et al. 2014). However, Kullmann (2014) notes the limits of hyper-realism for representing landscape designs because digital media, and the necessity of skills to use them, which increase the distance between the producers and viewers of the image. Instead, 'loose-realism' techniques such as digital freeform collages and montages should aim to retain control over the medium whilst enjoying the benefits of its modern visual styles. (Kullmann 2014).

The nature of participatory processes not only consists of consensus-driven facilitation (Schroth et al. 2011; van den Brink et al. 2007) but also of more antagonistic democratic processes (Laclau \& Mouffe 2014). This entails that the use of representations as 'communication tools' in participatory processes is not singular: the design image is not only a means to arrive at consensus but also a podium for explicating differences. Against this background it seems legitimate to conclude that the use of landscape design representations in planning and design processes revolves around questions of interactivity and power (Petrow 2011; Raaphorst et al. 2017). Although many studies deal with the power of specific types of spatial representations, such as maps and digital cartography (Bueno Lacy \& Van Houtum 2015; Crampton 2001; Harley 1988; Pojani \& Stead 2016), decisionmaking tools (Bishop et al. 2013), and interactive media (Salter et al. 2009), no known studies locate that power within the process of landscape visualization production, i.e. how and when planners and designers attribute what kind of discursive power to what aspects of design representations. By taking such a perspective we consider creating visual design representations to be a powerful discursive act, i.e. the construction of specific knowledge with a certain authority and credibility (Rose 2012).

In this paper we understand landscape design representations as discursive materializations of power and knowledge by studying the social relations within which the production of representations is embedded and how these relations manifest themselves in the communicative qualities of design representations. We assume that these relations determine whose knowledge is being represented 
with what kind of truth claim. Our research question is: What constitutes the discursive power of visual landscape design representations and how is that power used by planners and designers to facilitate communication during participatory design processes?

To answer this question, in this paper we present a visual discourse analysis of the landscape design representations and participatory planning and design processes which were part of Rebuild by Design $(\mathrm{RbD})$. $\mathrm{RbD}$ was a design competition organized in the wake of Hurricane Sandy that struck the greater metropolitan area of New York in 2012 (Ovink 2017). The RbD processes that took place were explicitly participatory and involved a diverse range of experts and participants. The projects resulted in a wealth of written and visual materials that describe the organization and outcomes of the design processes.

The structure of this paper is as follows. First, we introduce a conceptual and analytical framework for the study of visual discourse. This results in a classification of different discursive functions of design representations. In the subsequent section we analyse these discursive functions by means of design representations that were produced during three Rebuild by Design processes. Finally, we address the issue of planning and design as a situated practice, i.e. the kinds of discursive power that function through different types of representations at different phases of the design process.

\section{$\triangle$}

\subsection{Conceptual framework}

Visual landscape design representations play an obvious part in the process of visual communication: design content is expressed visually and interpreted by an audience. During this process, different types of knowledge are formulated as, for example, a design analysis, a spatial vision or a design solution, expressed using various visual modes presented on a particular medium. As such, a visual design 
representation has three main communicative elements: its medium possesses a level of interactivity, its visual mode provides a degree of readability, and its content is arranged in such a way it provides a certain validity (Figure 5.1) (Raaphorst et al. 2018). Design representations are interpreted by different audiences in different ways because every interpreter has a particular 'interpretive habit' (Raaphorst 2018). These habits consist of discursive networks, or strings of connotations, that are triggered by a design representation or particular aspects of that representation.

Actors and institutions use different means of visual expressions, and thus different levels of validity, readability and interactivity, to make a 'claim to truth'

\section{Representational Iconographical}

Pragmatics Interpretation

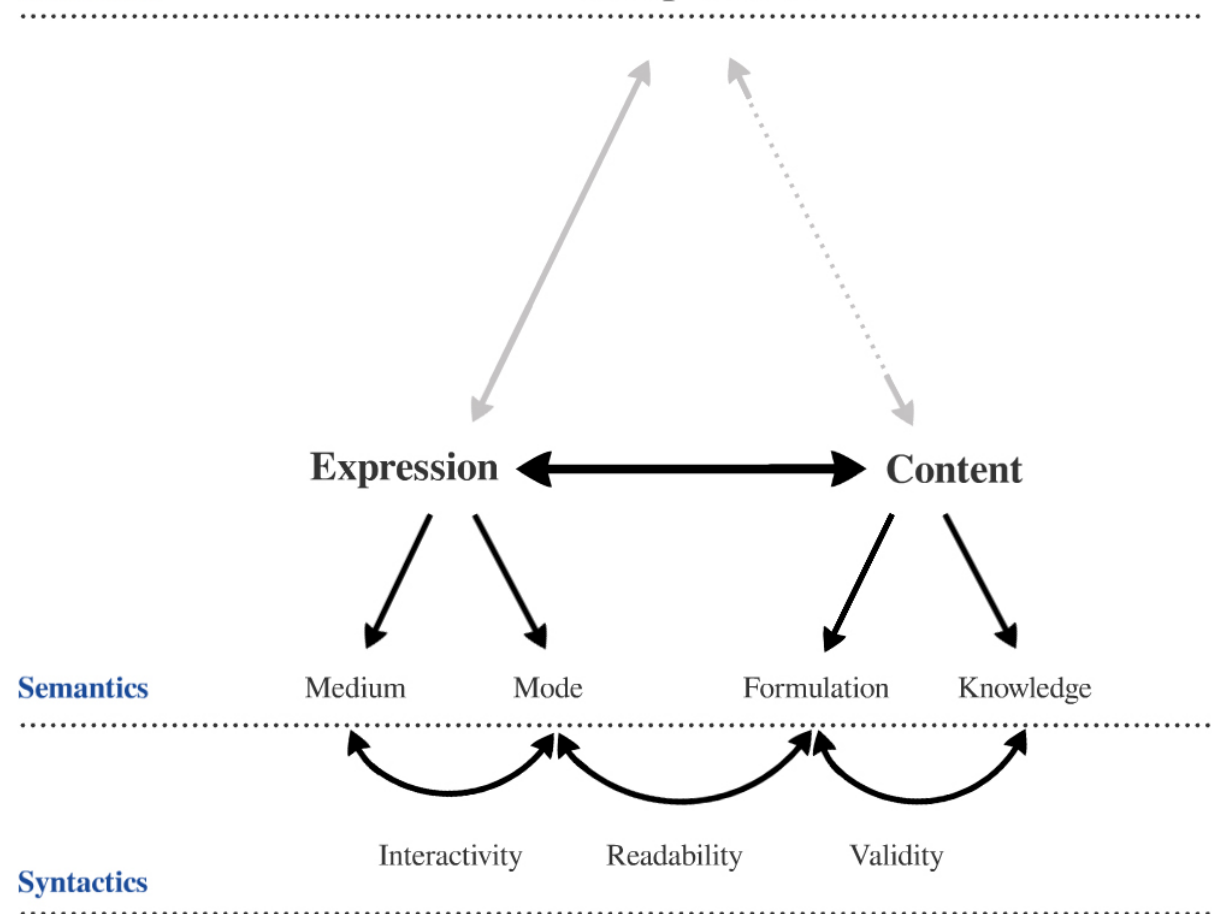

Figure 5.1. Conceptual framework for landscape design communication (Raaphorst et al. (2018). 
and solidify their knowledge, interests, and preferences during a design process (Crampton 2001; Dorling \& Hennig 2015). Such truth claims are made by attempting to influence the 'habits' or discursive networks through which audiences interpret design representations. Truth claims made through visual expression are defined as utterances of 'visual discourse' (Rose 2012). Visual discourse is inscribed upon an image via three key aspects during its creation: the institutional apparatus, the institutional technology and the rhetorical organization (Rose 2012). Elements of the apparatus are, for example, '... institutions, architectural forms, regulatory decisions, laws, administrative measures, scientific statements, philosophical, moral and philanthropic propositions' (Foucault, 1980: 194) that serve as strategies or relations supporting and supported by specific types of knowledge. Technologies consist of the 'practical techniques used to practice that power/ knowledge' (Foucault, as cited by Rose 2012: 230). The rhetorical organization of discourse can be uncovered by studying key themes, complexity, contradictions, the invisible as well as the visible, and their effects of truth (Rose 2012). For this study, these three aspects of discourse are conceptualized as the arrangement of the participatory process (apparatus), the interactivity provided by the medium on which the image is presented (technology), and the visual rhetoric of the image determined by the validity and readability of the design representation (rhetorical organization) (Figure 5.2). These conceptualized aspects (Figure 5.3) are used to structure the analytical framework of the visual discourse analysis conducted in this study.

\section{Arrangement of the participatory process}

In the context of visual discourse the level of participatory arrangement 'sets the stage' for visual design representations to function. In the course of participatory processes certain forms of knowledge become more dominant at the cost of others (Janes 2016). Landscape planners and designers, as facilitators, have the ability and opportunity to govern the extent in which these knowledge hierarchies are 


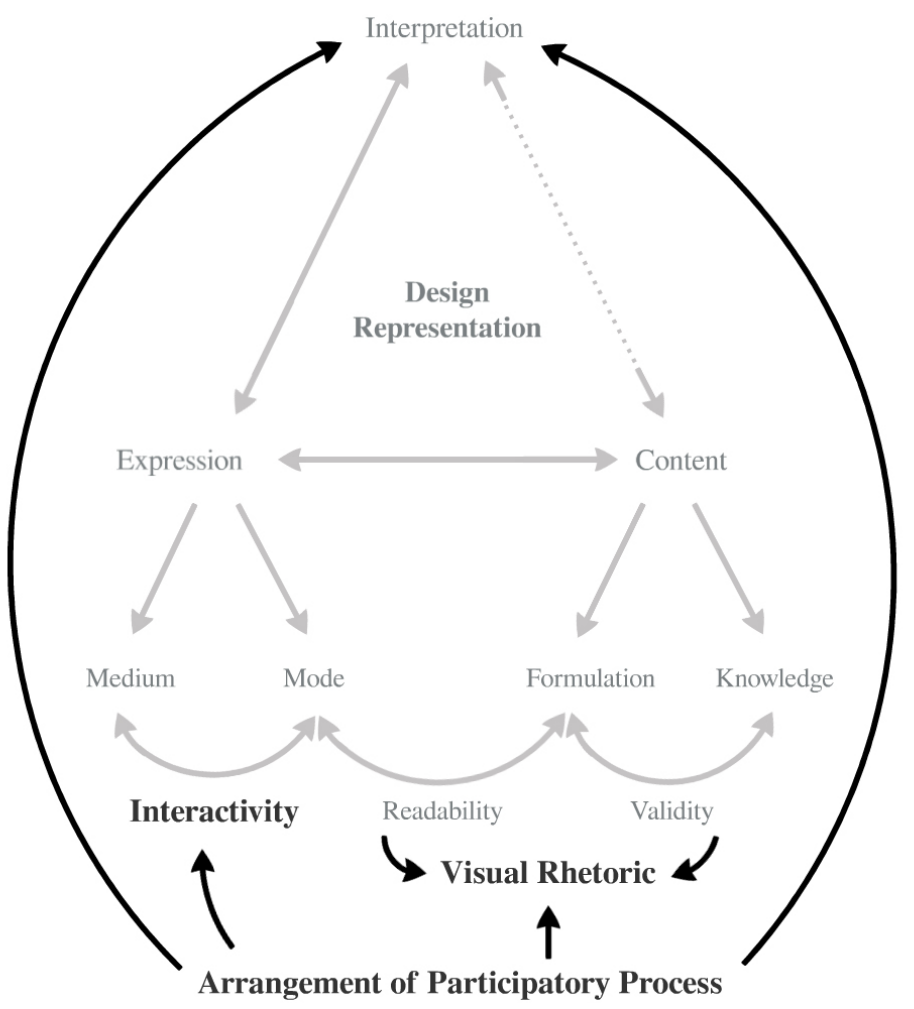

Figure 5.2. Conceptual framework for visual discourse in landscape design representations (Adapted from Raaphorst et al. (2018)).

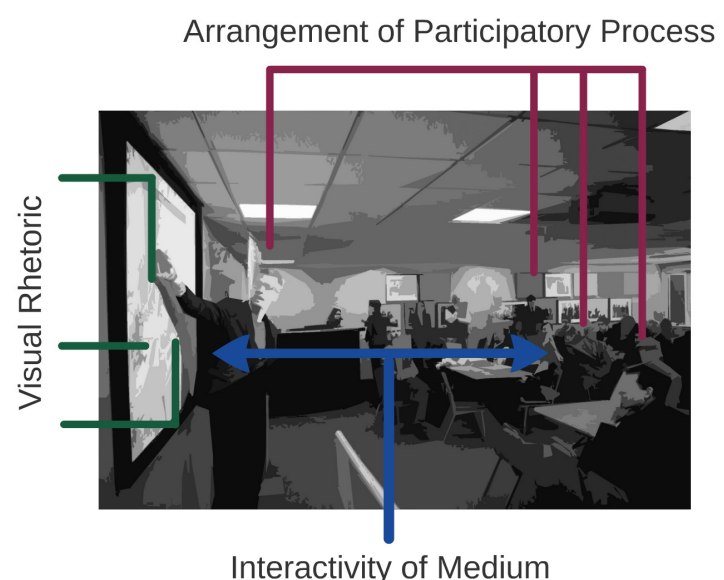

Figure 5.3. Three conceptualized aspects of visual discourse for participatory planning and design. (Image adapted from MIT-CAU et al. (2014)). 
reproduced and made productive. A concept that helps us to understand that ability is 'governmentality' (Foucault 2000). This concept refers to the governing abilities of planners and designers at all levels of abstraction, i.e. not only at the level of stakeholder groups, but also of individuals, relationships and visual representations. To study 'governmentality' means to examine to what extent planning and design practices aim to influence and govern the behaviour of others and 'the self' (Huxley 2008). For example, planners and designers are often able to choose who to invite, in what capacity, at specific moments in the design process.

Such practices can be called 'truth regimes' which are made possible by the 'invention and assemblage of particular apparatuses and devices for exercising power and intervening upon certain problems' (Rose, 1999: 19). For landscape planners and designers these apparatuses consist of the physical and social surroundings of design sessions they can choose, such as a community centre, design office or municipal hall. An apparatus can also consist of the regulative powers (Foucault 2000) that govern the degree of participation if and when planning and design processes are organized according to specific principles set by a commissioner, bill of law, or a design competition brief. Planners and designers attribute a certain role, level of influence, and authority to the participants they invite. The role of 'expert' or 'layman' can then be internalized by the participant depending on how he or she is involved, addressed, and valued (Janes 2016). The control over participation is thus exercised through many different tactics and strategies that ensure a legitimate mandate for the process organizers to eventually act upon the outcomes of the processes they set in motion.

\section{Interactivity of the medium}

All design representations that are produced during participatory processes can be powerful tools that enable and disable certain knowledge, forms of power, and visions of the future (Raaphorst et al. 2017). Landscape planners and designers are able to make this intersection between power and knowledge happen through 
the interactivity of the media they use and control. This interactivity can be both productive and constraining; the dominance of one discourse over another is established when the knowledge claim embedded in one discourse appears to be more 'true' than the other (Foucault 1974). Different forms of power need to be enabled to come into play because some discourses are more dominant than others, and the medium that planners and designers use has consequences for the forms of power it enables. For instance, a paper sketch offers a type of adaptability of design content that a projector screen cannot. At the same time, accessibility, or the lack thereof, might allow for the reproduction of local conflicts or other inequalities in local communities (Janes 2016). For example, although a paper sketch could invite people to draw, this does not necessarily mean people are able to draw, be it in terms of drawing ability or level of confidence.

The ability to share and construct knowledge is a form of power. According to Foucault (1980), power consists of any type of 'force', e.g. expertise, technical skill, political influence and charisma, that shapes our knowledge of the world (Foucault 1980). Different forms of power enable different participants to share their knowledge and expertise during planning and design processes (Janes 2016). As power shapes the knowledge that is embedded in landscape designs it also depends on that knowledge to function. The visual discourse expressed through the interactivity of media can thus exercise control over the message of the image (Sletto 2009), the extent in which knowledge can be shared and by whom, how that knowledge is materialized, and what kind of authority that knowledge has.

\section{Visual rhetoric}

Images represent their content with a distinct normativity that is obscured by the presumed objectivity of their visualization. This process of 'subjective naturalization' occurs through conscious and unconscious use of visual rhetoric (Barthes 1977). This means that the way planners and designers visualize design ideas. i.e. through the use of style, composition, colour scheme, text etc., expresses 
a certain subjectivity in a 'natural' way. According to Barthes (1977) these layers of visual rhetoric consist of linguistic, denotative, and connotative messages.

A linguistic message consists of textual elements that serve as an anchorage and relay. The anchorage of design representations describes the content of the design by means of a title, subscript, and captions. It directs the audience to specific elements of the image on the 'correct' level of perception. Textual relay points towards a direction or order of succession in which images or parts of images are perceived, such as the arrangement of text and images on a poster presentation.

A denotative message consists of the visual elements that make up an image. Those elements are 'coded' in a certain way (Barthes 1977) because an image cannot be a 1:1 depiction of a current or future reality. Planners and designers use representational codes, such as colour, perspective and scale, to approach that reality to a certain extent. The use of representational codes implies a simplification and therefore a selection and highlighting of certain aspects of a design. Each type of image represents, simplifies and selects its content in its own way. The more 'realistic' an image looks the fewer representational codes of simplification, selection, and technique are observed. For example, the cross-section of a dike shows very specific technical aspects of a flood defence structure while a photomontage shows the visual impression of a dike in its surrounding landscape. Moreover, visualization techniques, e.g. drawing by hand or on a computer, bind the denotative meaning of a technique to an image. For example, if a dike is depicted both by a photomontage and a GIS map the technique of representation ensures that the denotative meaning of those images differs. While a photograph, and therefore also a photomontage, could present a 'natural being-there of objects' (Barthes 1977), a sophisticated GIS map grants the depicted information a geographical and even mathematical credibility. In these cases, the denotative messages naturalize their symbolic meanings by depicting a designed future dike structure photorealistically and by presenting information about that structure with authoritarian accuracy. 
A connotative message consists of the possible interpretations of ambiguous denotative codes. In this sense, visual rhetoric consists of the specific composition or interplay of visual elements that invokes additional meaning compared to the described denotative message. For instance, the use of specific styles or techniques and the depiction of weather conditions influences the connotations attached to a design representation. This connotative 'surplus' can create a discontinuity between the written descriptions and explanations of a design's qualities and the visual elements that make up the design image. A study of the visual rhetoric of design representations is achieved by inferring the connotative message of the image by means of its anchorage and by relating the acts of representation, simplification, selection and the authority of its visualization technique back to its denotative message (Barthes 1977).

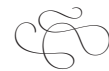 \\ 5.3 Visual discourse analysis}

The conceptualized aspects of visual discourse, i.e. the arrangement of the participatory process, the interactivity of the medium and the visual rhetoric of the image, are operationalized into the analytical categories depicted in Table 5.1. These categories constitute the analytical framework used to study examples of different participatory settings that occurred during the planning and design processes of the Rebuild by Design competition $(\mathrm{RbD})$ and the visual materials produced during these processes.

\section{Rebuild by Design}

In its mission statement (Ovink 2017), the RbD organization advocated an holistic and inclusionary perspective on the planning and design of resilient flood defence solutions. The different project consortia consisted of, among others, planners, designers, engineers, hydrologists and geologists, that were required 
Table 5.1. Visual discourse analytical framework.

\begin{tabular}{ll}
\hline Visual discourse component & Analytical categories \\
\hline Arrangement of participatory process & Physical setting \\
& Social setting \\
& Regulative powers \\
& Accessibility of design content \\
Interactivity of medium & Adaptability of design content \\
Visual rhetoric of image & Anchorage and Relay \\
& Denotation \\
& Connotation \\
\hline
\end{tabular}

to establish productive relationships with local citizens, businesses, NGO's, politicians and legislators through participatory approaches to attain strong public and political support for the implementation of the process outcomes (Rebuild by Design 2013).

For this paper, three of the winning $\mathrm{RbD}$ proposals (Table 5.2) serve as embedded case studies within the $\mathrm{RbD}$ competition. This selection of projects is based on feasibility in terms of data collection, i.e. good availability of image materials and the availability of interview respondents. The projects and locations depicted in Table 5.2 cover a wide range of landscape scales, types, flood risk challenges and stakeholder configurations.

Each project team provided a detailed report of their design process as part of the submission for the RbD competition. Each report (Interboro 2014; MIT-CAU et al. 2014; OMA 2014) contains a detailed analysis of the project area, an actor analysis of important stakeholders, an overview of the workshops and sessions that were organized, design concepts of proposed design solutions as well as more concrete

Table 5.2. Overview of RbD proposals used for visual discourse analysis.

\begin{tabular}{lll}
\hline Project Team & Project Title & Project location \\
\hline OMA & Resist, Delay, Store, Discharge & Hoboken, NJ \\
MIT-CAU+ZUS+URBANISTEN & The New Meadowlands & Meadowlands, NJ \\
Interboro & Living with the Bay & Long Island, NY \\
\hline
\end{tabular}


plans for phasing and implementation. Besides the documents of the submissions, 16 interviews were conducted between 2015 and 2016 with landscape architects and urban designers, a city planner, an organizer of public outreach processes, a real estate developer, a leader of an environmental protection agency, academic experts and other stakeholders of the project teams of the three $\mathrm{RbD}$ proposals. These interviews were transcribed, coded and analysed using Atlas.TI software.

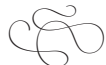 \\ 5.4 Participation, Interactivity, and Rhetoric}

In this section examples are presented from the selected $\mathrm{RbD}$ projects wherein the arrangement of participation, the interactivity of the medium, or the visual rhetoric of the image has been purposefully used by the designers to facilitate the planning and design process in a certain way. Three distinct phases or moments in the design competition process are distinguished: the analytical stage, the codesign stage, and the final presentation stage. The data inventory techniques that were used by the Hoboken project team illustrate the analytical stage of the design competition. The participatory drawing techniques used by the Long Island project team describe the co-designing stage. Finally, the visual rhetoric of the Hoboken project and the poster strategy used by the Meadowlands project team exemplifies the final presentation stage of the design proposals.

\section{Inventory analysis: Hoboken, NJ}

In the analysis and inventory stage of the $\mathrm{RbD}$ process the design teams aimed to gather information on their project area, make an inventory of local knowledge and explore potential design solutions. In the beginning of the Hoboken project the arrangement of the participatory process was focused on education: 


\section{Member Community Advisory Group:}

"Consider an "awareness, education, and engagement" spectrum (...) at this whole end of the spectrum of awareness, people don't understand the problem, therefore they cannot even envision a solution, along that spectrum, once you get people aware, you need to try and engage them in something and that's where participatory design comes in.'

In service of that educational process the project team came up with several techniques to facilitate participation. For instance, the team developed a pamphlet (Figure 5.4) containing real-life examples of statistically relevant events in service of raising flood risk awareness:

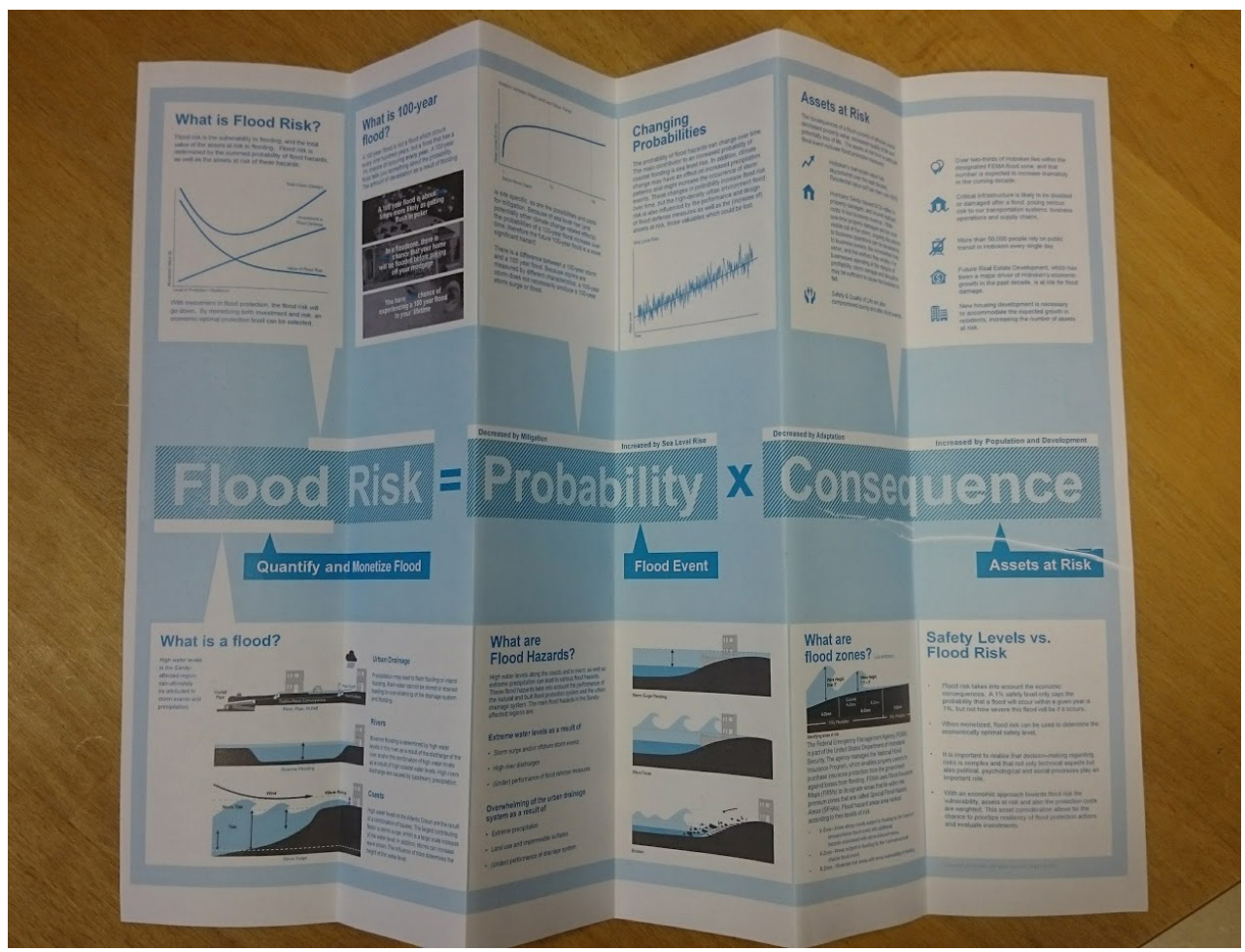

Figure 5.4. Flood risk pamphlet produced by the Hoboken project team (OMA 2014). 
Lead architect:

'People were making assumptions that Sandy is a 1 in 100 years event, so they don't have to worry for another 100 years (...) but a 100 year flood is about five times more likely as getting a flush in poker. (...) Tying it to a mortgage, in terms of buying a house which is an event that most people go through and it has a time scale within a kind of single life time (...) what is the frequency of the said event during that kind of time period? (...) We felt it helpful, both in our work with stakeholders but also internally (...) being able to quantify risk and be able to monetize it to a degree people could understand.'

A pamphlet is a passive medium, i.e. it presents information as facts to be perceived and remembered by its audience. In this case, it was used to establish a baseline knowledge level to increase people's awareness of flood risk.

Once the relevant stakeholders were aware of the severity of the risks they were exposed to it was time to start thinking in terms of possible solutions. An educational game was introduced to help people understand the possibilities for combining different flood defence options, as the designer from OMA further explains:

'(...) a game where people had 10 kinds of tokens to invest in resist, delay, store, and discharge measures so they can understand the trade-offs between the different components.'

This kind of game uses an active medium with no preferred goal, i.e. there is no 'one best way' of distributing tokens. Rather, the goal was to make people understand the complexities and interdependencies of the different strategies that were proposed. Once a shared understanding of the risks, problems, and the feasibility of potential solutions was established the team attempted to engage with 
a larger public group to explore the attainment of public support for the different design options:

Rebuild by Design assistant organizer:

'(...) during the public comment period you had comment cards that were already stamped and addressed, so you just have to write what you want, tape them closed, and send them, you just put them in the mail.'

Such a 'take home' approach establishes a form of interactivity and participation that is different from regular voting procedures. People are able to think, discuss and evaluate design ideas at home and at their own leisure without the necessity of providing immediate feedback, which is sometimes resisting and conservative.

\section{Co-designing on paper: Long Island, NY}

In the co-designing stage the arrangement of the participatory process was aimed at designing together with local stakeholders to iteratively calibrate design concepts to the preferences of project participants. Workshops were organized where the designers presented their initial ideas, followed by breakout sessions to adjust and improve those ideas. The medium used, and the interactive technology bound to that medium, influences the 'truth status' of the content that is created during such participatory workshops. For instance, one of the lead designers of the Long Island project team explains how drawing on paper is preferred to using a GIS:

Lead designer:

'You should start by drawing, in that way the whole situation is not so threatening and more related to the origin of the ideas. If you use a digital Maptable to draw a line, that line is immediately made part of a GIS: it becomes exact. But if I cover up 
that Maptable with transparent paper, use a big marker, and draw a line across 20 houses, it doesn't matter because it's just a sketch.'

Not only the perceived legitimacy of the representation was a factor for them to opt for an analogue medium, the mobility of digital technologies played an important role as well:

'Using a GIS that line becomes part of a model. You can make a handout and take it home with you. In the case of a sketch you can remove the underlying map and all that remains is an arrow and a stream, and people will agree: "yeah that seems about right". It's not threatening.'

A paper sketch allows the status of its content to be controllable. In this case the content entailed preliminary ideas which were part of an ongoing design process and which were not meant to leave the room. This aspect of controllability intertwines with the visual rhetoric of a paper sketch. For instance, the anchorage of a paper sketch is highly situational; often there is no title or legend added to the sketch. Instead, the meaning of a sketch is shared between the participants that were present when it was created:

'If you're part of the creation process and the act of drawing, you remember that the next time you're there.'

During a participatory drawing session the 'connotative surplus' is kept to a minimum since each point, line or shape denotes that which is agreed upon at the moment it's being drawn. The rhetoric of a sketch thus functions by virtue of its interactivity and participatory setting. A different audience is left with no clear instructions to decode the image if such a sketch were to be transported outside its original context without the necessary anchorage to denote its meaning. The 
example of Figure 5.5 illustrates how a sketch without anchorage is 'transported' into the project report by adding a caption, a base map for orientation, and a colour and texture scheme. The caption is necessary to provide anchorage for the elements that represent sediment management processes.

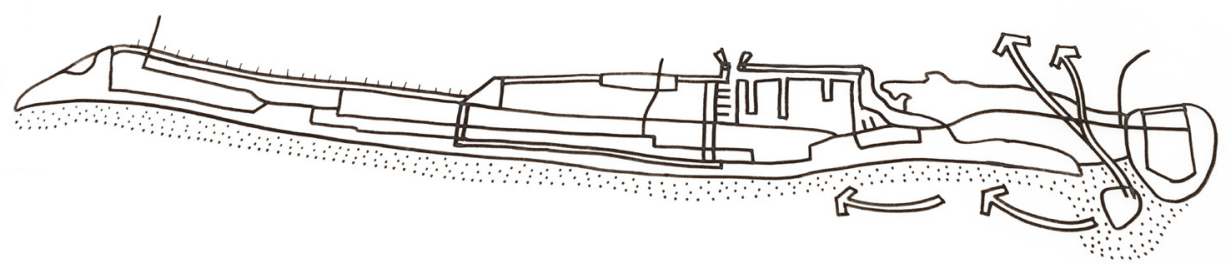

Figure 18. The implementation of the strategy for resilient sediment management within Nassau County results in a broad ocean beach with dunes. The relocation of the Jones Inlet (between Long Beach and Jones Beach) toward the east would allow the accumulated sediment to nourish the eroding beach of Long Beach. A wash over between Point Lookout and Malibu Park would allow more sediment to be transported toward the bay. Nourishment of the ebb-tidal delta would provide sediment for the coast and the bay on the long term.

Figure 5.5. Paper sketch without anchorage (top) vs. digitized drawing with anchorage of later iteration (bottom) (Interboro, 2014).

\section{Presentation: Hoboken rhetoric}

The design ideas that are part of the Hoboken project consist of a diverse collection of proposed flood adaptation initiatives located throughout the city. The team came up with the strategy of 'resist, delay, store, discharge' in need of a unifying rhetoric. The lead architect of the Hoboken project explains:

'(...) the most important thing was creating a strategy that was well communicated and that served as a kind of umbrella or framework for the city to not only push forward with our (design office - authors) recommendations, but a host of other initiatives that they (the city - authors) were doing under resiliency.' 
This strategy constitutes the main anchorage with which the different design concepts are categorized, either as barrier (resist), as increased permeability of the street surface (delay), or as catchment (store) and transportation devices (discharge). This anchorage is used throughout the reports and presentations of the design team and connects a variety of images of different visual styles and techniques (Figure 5.6). Such a comprehensive strategy appropriates the denotative elements in the images in terms of their flood defence functionality. The top image (a) denotes the locations where this functionality is needed, the middle image (b) shows how such functionality can be visually embedded in the urban landscape, whilst the bottom image (c) denotes the relative contribution of each principle to the attainment of a desired level of flood safety.

A consistent anchorage creates consistency on a conceptual level, e.g. 'Resist, Delay, Store, Discharge' in Figure 5.6. This figure however, is composed of several images which differ in visual form, creating a 'connotative surplus' of many non-comparable visual elements. In the absence of proper additional anchorage this inadvertently leads to an increased variability of connotative messages. For instance, the middle image (b) provides impressions of four types of interventions, yet not all of the proposed interventions at the locations that are depicted on the map will share those specific spatial characteristics. For example, the design proposal explains that the interventions of the 'store' category consist primarily of underground pipes and valves that retain and transport excess water towards its 'discharge' locations (OMA 2014). However, based on the impression in the middle image of Figure 5.6, a connotation could be that these wide, green infrastructural elements will be placed throughout the city. Moreover, the 'store' measures connote a high degree of importance as a visually dominant element in the top image (a). Yet, that importance is not reflected in the bottom schematic drawings considering the relatively small part of the 'store' measures for reducing the chances of a flood in Hoboken. 


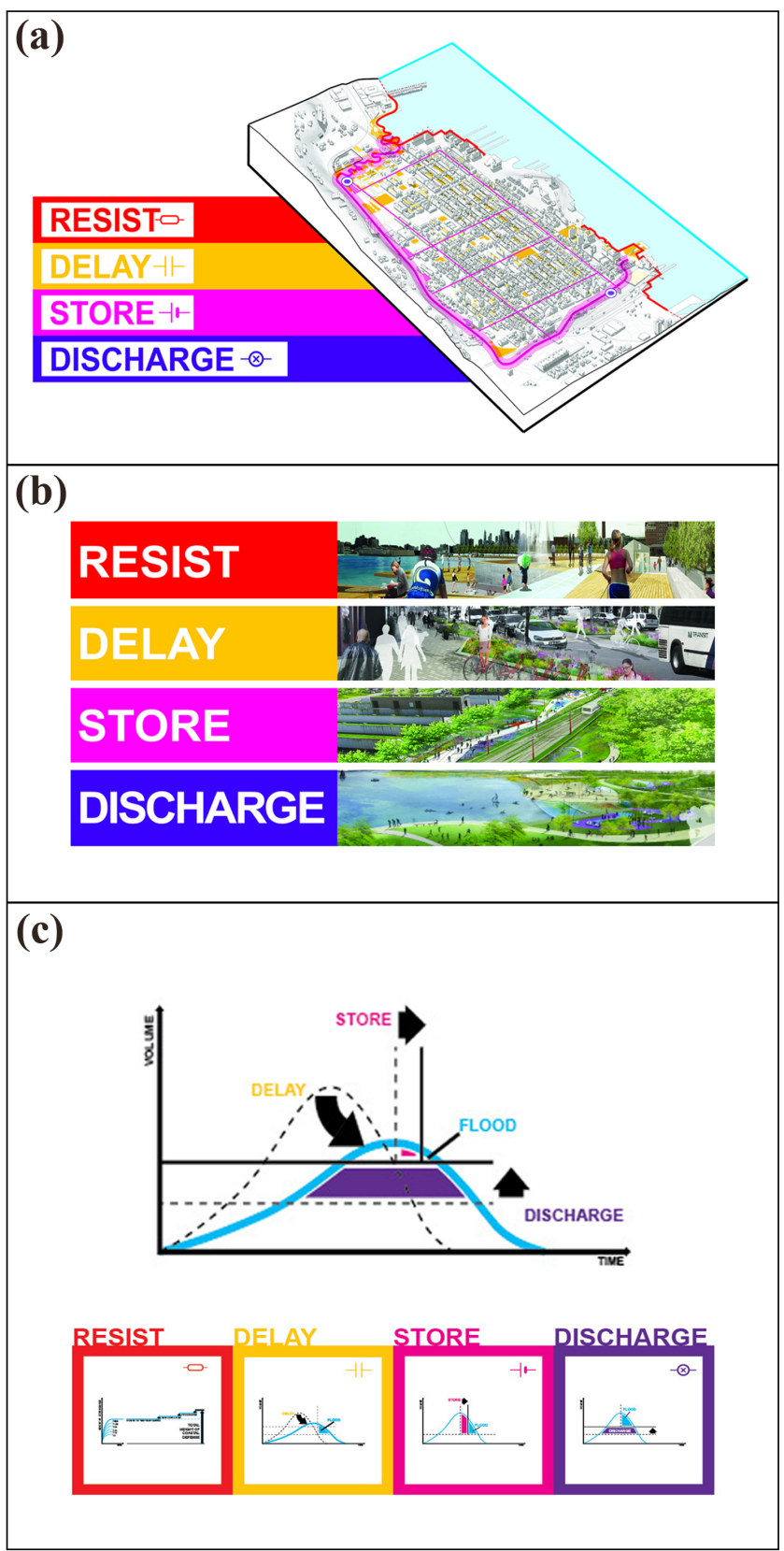

Figure 5.6. Visual rhetoric: examples of the 'resist, delay, store, discharge' rhetoric in the Hoboken design proposal (OMA 2014). 


\section{Presentation: Meadowlands poster strategy}

An 'open market' was organized on the eve of the design competition's finale. At this stage, the design concepts were finalized, the materials were printed and the presentations for the jury were prepared. The participatory arrangement of this event was meant to offer the general public, the other design teams, and $\mathrm{RbD}$ organizers a first impression of the various projects. Simultaneously, it enabled the design teams to gauge the first reactions of a potentially wider audience. One of the designers of the Meadowlands team describes the physical and social setting as follows:

'The day before the (final) presentation we had an open market. We could present our ideas, or ask questions (...) The ten proposal boards were in a big room, with a lot of people coming in, with some drinks, a nice environment. We had to "catch attention".'

The participatory arrangement at this particular moment materialized in the form of an exhibition wherein the goal of the teams' presentations was to catch people's attention and to promote and explain the design proposals. The poster boards themselves provided a low level of interactivity; the content of the poster not adaptable by the audience, and the accessibility of the posters is fixed in the setting of an exhibition. This means that the poster boards were not interactive in the sense that the public could add or adjust their contents.

The Meadowlands team attuned the medium and visual rhetoric to the type of interaction the 'open market' setting had to offer. The representations and media they used consisted of a poster board (Figure 5.7), an abstract scale model, and a comprehensive 'coffee table book' that contained a detailed report of the whole planning and design process. This combination of media provided a dynamic between the designers and their audience that exceeded the static setting of an exhibition, as one of the designers explained: 
'We made a poster board, containing a cross-section, with the three concepts of protect, connect and grow. The idea behind it being it was more or less the complete idea of the plan but simplified. (...) Many people were looking at the boards, the book, and the model, and they said: "Do you have something in between? Not as abstract as this (model-author), but not as dense as this? (book-authors)" And I said: "I am the one to talk to".'

The Meadowlands proposals attracted a considerable crowd compared to the other design teams because of this combination of media. According to one of the designers the interactivity that was achieved, namely the triggering of the audience to engage in conversation with the designers, enabled the project team to explain their ideas face to face:

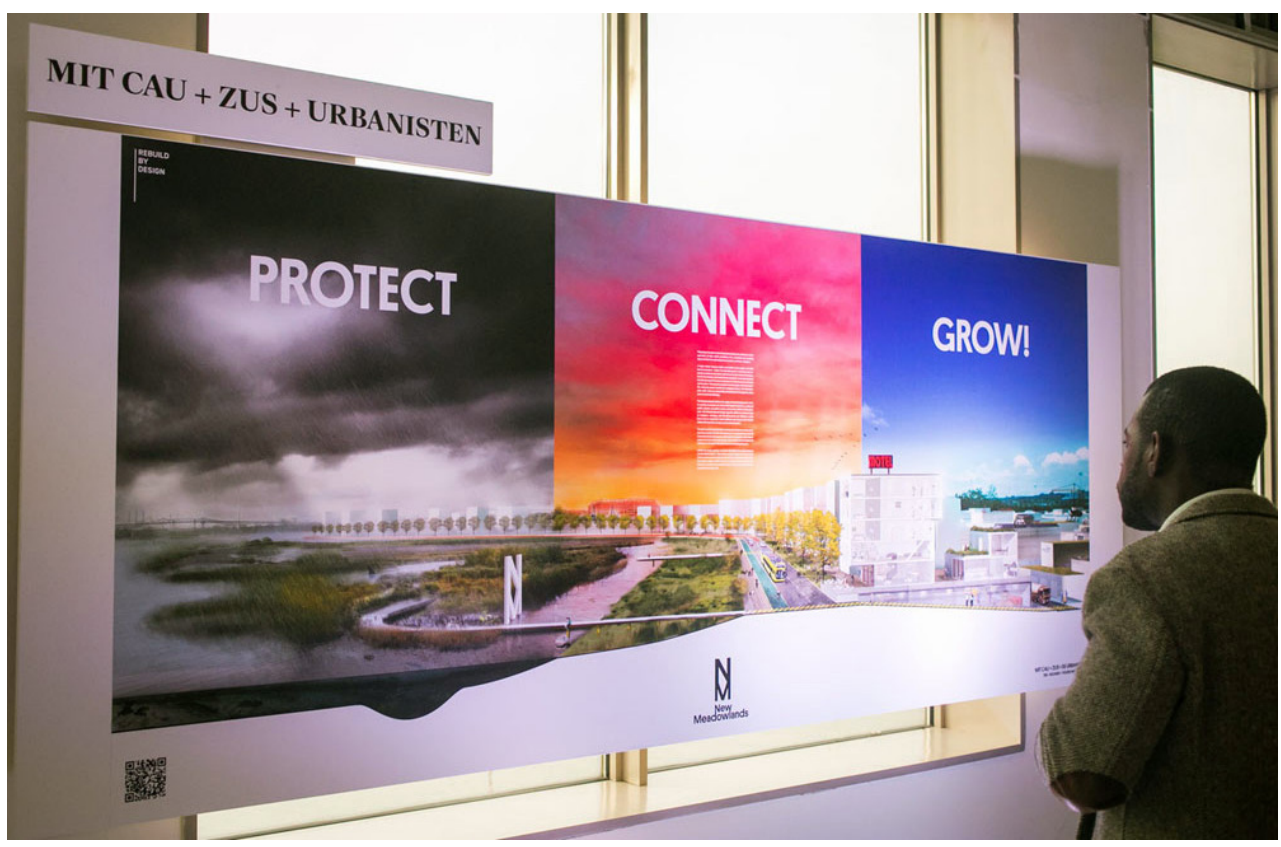

Figure 5.7. Poster boards of the Meadowlands project team. (MIT-CAU et al. 2014). 
'Our presentation drew a large crowd. People saw this beautiful rendering, and wanted to know what's happening. They came up to us with questions, and it was very nice to explain to people what the plan was about. And that was really easy to do: taking them through the whole story using the scale model for reference.'

The visual rhetoric used for the poster board served a specific purpose, especially in relation to that particular medium. As the designer from the New Meadowlands project further explains:

'We didn't have to explain what there is to see on these tiny images or graphs, like it is the case with these other posters (See Figure 5.8 for reference-authors). And the thing that also worked was that this style combined the technical story with the attractive story. We actually tried to turn it into a "happy" landscape, where people could envision themselves jogging, etcetera. And it worked.'

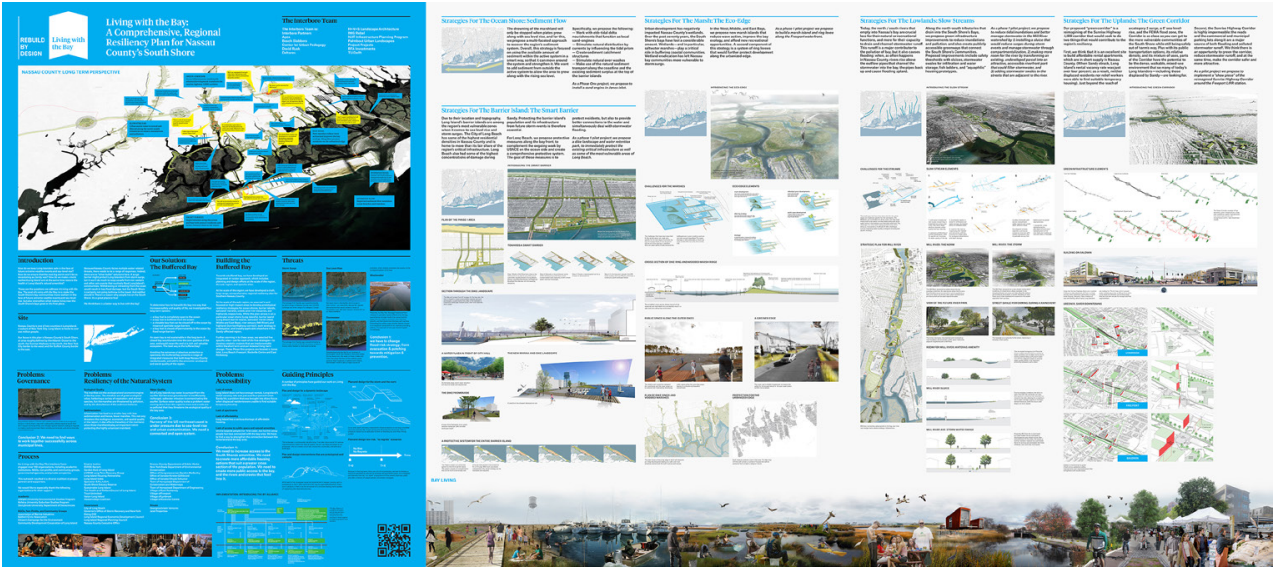

Figure 5.8. Poster boards of the Long Island project proposal (Interboro 2014).

The textual anchorage encompasses the design strategy and consists of three words and a text box in the middle of the poster that summarizes the main ideas. A relay is established through font-size, i.e. the strategy is readable from a distance whilst the text box requires a closer look. However, the majority of the anchorage 
is provided verbally by the designers whilst they engage in conversation with their audience. In doing so, the denotative meaning of the image becomes fluid: the designers can add or adjust the 'verbal' anchorage to the conversation they have. In this way, the designers consciously manipulated the social setting of the presentation event by increasing the interactivity of their representation.

However, the connection between the denotation of the image and the medium on which it is presented is crucial once the verbal anchorage is taken out of consideration. For instance, the project report becomes the sole anchorage that explains the denotative meaning of the image when the poster boards and the project reports are distributed through other communication channels such as the internet. The connotative signs that refer to the aforementioned 'happy' landscape are no longer grounded, as the mental image of jogging is not a functional component of the design. As such, the connotative surplus of the happy landscape can create a discontinuity with the functionality of the design.

\section{$\nabla$}

\subsection{Conclusion and discussion}

The aim of this paper is to understand landscape design representations as discursive materializations of knowledge and power. The analytical framework that is presented identifies three components of design representations that have a discursive functionality, i.e. the visual elements of the image that constitute a visual rhetoric, the interactivity of the medium, and the arrangement of the participatory setting wherein these representations are created and perceived. The analysis presented depicts three cases of participatory arrangements, i.e. analytical workshops, co-design drawing sessions, and final (poster) presentations. In each case, the use of specific media and their interactive qualities supported the level of participation that the designers wanted and the visual rhetoric operated in service of the participative setting for which it was created. An important interdependence 
between the three categories of visual discourse is found. For example, the interactivity of the Hoboken token-game enhances the level of participation by improving the degree of understanding of design possibilities; the rhetoric of paper sketches only functions by virtue of a distinct participatory setting and drawing ability; the interactivity of a poster board is complemented by the setting of an 'open market' wherein designers can engage their audience verbally.

The discursive power of images shapes interpretive habits of audiences towards its own truth claim. As such, this paper illustrates that planners and designers are able to use that discursive power in at least two ways. A first strategy entails the alignment of the participatory setting, media interactivity and rhetoric of images with the expectations of participants to assure specific forms of knowledge construction, evaluations of the process, and validity of the designs that are produced. An example of this strategy is the use of analogue drawing techniques during co-design sessions by the Long Island project team. The second strategy entails changing the expectations of participants through the use of specific participatory settings, media interactivity and visual rhetoric, to unite participants' preferences with the anticipated vision or result of the process facilitators. Examples of this strategy are the flood risk pamphlet and interactive game of the Hoboken team that aimed to increase the awareness and understanding of flood issues by local participants, but also the unifying rhetoric of 'resist, delay, store, discharge' to integrate existing municipal initiatives into new design ideas.

The aforementioned interdependence between participatory arrangement, media interactivity and visual rhetoric is demonstrated to be an important prerequisite for visual discourse to function. For instance, co-creation cannot occur when planners or designers use a medium that does not allow the design content to be adjusted. Moreover, visual rhetoric might connote unintended meanings and give rise to counterproductive interpretations amongst audiences once an image is taken out of its original context. Similarly, the chances of misinterpretation increase when a different rhetoric is used to visualize the same content. 
In hindsight, a complementary perspective on the interdependence between elements of visual discourse can be found in the theory of 'resemiotization' (Iedema 2001; Scollon 2008). Resemiotization 'is about how meaning-making shifts from context to context, from practice to practice, or from one stage of a practice to the next' (Iedema 2003: 41). For instance, the artist impression depicted on the New Meadowlands poster boards (Figure 5.7) was created specifically for the presentation sessions. This means that all of the design content that was developed up to that point was previously created, communicated, and stored using a variety of different media and different forms of visual rhetoric. The poster presentation might very well be the first time that project participants see the design expressed in such concrete visual terms; they might not fully recognize the earlier iterations of the design or their own contributions to the project.

It is important to note that resemiotization occurs through multimodal means, i.e. a combination of different semiotic modes, such as speech, gesture, and written text (Kress 2009). The creation of a visual design representation, with the intent of using that image in different design contexts, entails a transposition of multimodal content into a single self-explanatory image (Iedema 2001). For instance, a codesign workshop results in design sketches whose meanings are at least partly agreed upon by different participants. Proper anchorage and relay, e.g. title, legend, and date, is necessary for a 'basic' resemiotization of the content of the sketch. However, that anchorage and relay can never fully cover the intricacies of a participatory design session. Since design representations always function in a multimodal context the cause of a possible discursive effect can never be traced back to just one (visual) representation. So even though the discursive functions of design images are important tools of power, a broader multimodal study of how these images are created and subsequently used could be useful for arriving at a more complete understanding of the communication processes that occur during participatory planning and design projects. 
In this paper we studied the creation process of landscape design representations from the perspective of the 'production', i.e. the designers that facilitate co-design sessions. As such, the research presented here emphasizes the intention and vision of the planners and designers who made the design representations. However, the extent in which representations function as they are intended by their makers also depends on the interpretation of and evaluation by their audiences. For example, the mentioned 'happy landscape' of Figure 5.7 could easily be interpreted by someone who fears urban development in the area as 'unhappy'. Even though the designers that were interviewed were able to recognize the effect and use of their visual representations, a triangulation of those effects as they were perceived by their intended audiences could provide valuable insights into the reasons why certain strategies work better than others.

Visual discourse is consciously and unconsciously applied whenever design visualizations are created for a specific purpose with a specific audience in mind. Future research could be focused on purposefully creating design representations and subsequently monitoring their use and reception over time. Doing so could increase our understanding of the influence of specific visual discursive elements on specific audiences. Locating discursive power within the production of visual landscape design representations during participatory planning and design processes can therefore enhance awareness amongst landscape planners and designers of discursive power in their work. In turn, the discursive qualities of design representations can be used more productively in service of participation by minimizing miscommunication and consequently ensuring a broader inventory of local and expert knowledge and a more widely supported co-design process. 


\section{至 \\ Acknowledgements}

The research for this paper was carried out in the context of the research programme 'Multifunctional Flood Defences', funded by the Stichting Technologie \& Wetenschap (STW), part of the Netherlands Organisation for Scientific Research (NWO). Additional 'in-kind' funding has been provided by knowledge institute Deltares.

The authors would like to thank the anonymized interview respondents for participating in this research project. 


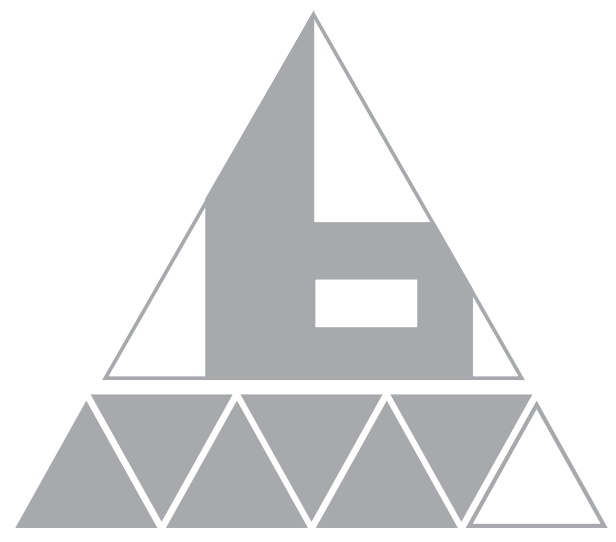





\section{Chapter 6}

\section{CONCLUSIONS AND DISCUSSION}

\section{$x$}

\subsection{Introduction}

This thesis investigated how the different meanings of visual design representations shape the progress and outcomes of participatory landscape planning and design projects. The study was triggered by the notion that spatial planning and design projects, especially in the light of climate change adaptation, increasingly call for multifunctional landscape solutions. This multifunctionality leads to the inclusion of both academic and professional experts as well as local stakeholders. Usually, each expert or stakeholder has a different perception of what the design challenges and solutions are, or could be, in different phases of the design process. As a result, the task of facilitating participatory planning and design processes becomes more complex and the ability of design representations - as primary means of communication that have different meanings to different project stakeholders - to shape these participatory processes becomes more important as well. Despite the centrality of visual representations within spatial design disciplines, a critical visual research approach to studying how the wide variability of analogue and digital visual representation techniques shapes participatory planning and design processes is lacking.

The context described above led to the following research objective: to contribute to a more conscious use of visual design representations in participatory landscape planning and design projects by studying the visual communication that occurs between designers, experts and stakeholders as meaning-making processes. Identifying the factors within the meaning-making processes that shape the progress and outcomes of participatory planning and design projects could 
enable more effective and transparent communication in the future. Therefore, the research question central to this thesis is as follows:

What meaning-making processes are visual landscape design representations part of, and how do these processes shape the progress and outcomes of participatory landscape planning and design projects?

In this thesis, the subjects of inquiry were (1) visual design representations in their many forms and appearances; (2) the meanings that are attributed to or derived from these representations by designers, planners, and project stakeholders; (3) the participatory planning and design processes of multifunctional flood defence landscape projects where these representations are used. To study these subjects I developed a critical visual research approach through the exploration and operationalization of semiotic and critical social theory and through the development and application of visual research methods. I then used this critical visual research approach to study the visual representations that were produced during participatory design processes that were part of Rebuild by Design $(\mathrm{RbD})$. $\mathrm{RbD}$ was set up as a design competition organized in the wake of hurricane Sandy for the design of multifunctional flood defence landscapes in the greater metropolitan area of New York. This design competition brought together landscape architects, planners, engineers, climate experts, politicians and local communities in a participatory setting. The visual communication processes that took place amongst and between these complex stakeholder configurations provided the case studies that formed the basis of this thesis.

I arrived at the answer to the research question of this thesis by studying the visual communication of landscape designs as meaning-making processes. This entailed studying landscape design representations from four research foci: the critical functions of design representations, the image, its interpretations by their intended audiences, and its production by planners and designers. In Chapter 2, a 
theoretical exploration provided the outline for a critical visual research approach in the field of landscape architecture to study the critical functions of design representations. The analytical framework of this critical visual research approach is the result of the synthesis of Chapters 3,4 , and 5 of this thesis. This framework depicts a semiotic conceptualization of visual landscape design representations with a focus on the image, its interpretations, and its production. In this conceptualization I distinguish - in Chapter 3 - between the semiotic complexity of the image and its content, the interpretative habits that guide the audiences' interpretations in Chapter 4, and in Chapter 5 the production of visual discourse, which influences those habits. I present and explain this analytical framework in the following section of this chapter, after which I introduce a typology of semiotic qualities that can be used to describe how the meaning-making processes of design representations shape participatory projects.

In the discussion section, I position the results of this study within the current academic debate on landscape design visualization and discuss the extent to which the developed critical visual research approach complements or contradicts the existing body of knowledge. Subsequently, I reflect on the research approach itself and on my role as a researcher in relation to the different subjects of inquiry. Lastly, I make recommendations for further research and indicate the societal relevance of this thesis.

\section{$\triangle$ \\ 6.2 A critical visual research approach}

The answer to the first part of the research question of this thesis involves the development of an analytical framework for a critical visual research approach to studying the visual communication of landscape designs as meaning-making processes. The main premise of this critical visual research approach is based on 
the potential of different theoretical views and visual research methods for making the implicit critical functions of design representations more explicit (Chapter 2).

Within this thesis's critical visual research approach semiotic complexity (Chapter 3, Figure 3.6) is studied from an iconographic perspective. Using such a perspective, semiotic complexity is studied systematically by following a specific semiotic vocabulary and categories of visual content. The 'semantic' categories used in this thesis, i.e. knowledge, formulation, mode, and medium, constitute the main sign components of landscape design representations. The relationship between these sign components helps to identify three internal 'syntactic' relationships or communicative elements: validity, readability, and interactivity. The validity of design ideas relates to the functionality, visual aesthetics and feasibility of the design. This element is determined by whether the design solutions are suitable for arriving at a multifunctional landscape design; whether these solutions are deemed necessary and implementable; and whether they are supported by the project stakeholders. The readability of a design image relates to the extent to which participants can read and understand the content of a design. This element is determined by whether the graphical techniques used, the colour schemes and perspectives, etc. match the visual literacy of the audience. The interactivity of a design image relates to the medium on which the image is presented. This element is determined by the extent to which that medium allows the participants to interact with the content and expression of the design.

The iconographic perspective helps to relate the visual content of design representations to its 'pragmatics' or original context: the moments of its production during the participatory process and the context within which it was interpreted. This method of visual content analysis enables the identification of 'semiotically complex' representations. This identification is useful for selecting specific images for the further qualitative analysis of the visual discourse embedded within them during their production and of the conflicting interpretations that their semiotic complexity could give rise to. 
Interpretations are conceptualized as the interpretive habits of different project stakeholders. The study of interpretive habits, as social semiotic discourses, explores how people construct their interpretation of a design image and whether that interpretation can be anticipated by the planner or designer. As a contextual analyst, the landscape planner or architect can create 'situational maps' of the sociopolitical contexts of participatory planning and design projects. These contexts are considered as networks of signs or discursive elements, discursive relations, nodal points and discursive regimes or formations (Chapter 4, Figure 4.1). A cartographical map, a legislative document, a natural disaster, a mayor or a design firm are all signs or discursive elements that can be attributed identities of value, agency and function within a discourse. Discursive relations between discursive elements embody the typologies with which one element can be distinguished from another. These relational typologies also lead to the identification of the elements that belong to the same discourse. Nodal points are signs that represent the shared identity of different discursive elements, e.g. a design intervention that encompasses knowledges from various disciplines, and that function as conceptual instruments that can be used to consider these elements as one entity. A discursive regime can be defined as the situated enactment, validation and perpetuation of a discourse or set of discourses by specific institutions through actors that are assigned specific social roles. Mapping the interpretive habits of specific participants enables a contingent anticipation of how the validity, readability and interactivity of a design representation can be evaluated and acted upon by different project participants.

The interpretive habits of audiences are considered as discursive networks that are constantly influenced by signs and sign systems that have discursive power. The production of design images is conceptualized in terms of this discursive power, i.e. the visual discourse. The analysis of visual discourse entails the study of how the producer of design representations can influence the ways in which these representations are interpreted (Chapter 5, Figure 5.3). A producer could 
be a landscape planner or architect but also a civil engineer, a hydrologist, or a local inhabitant, depending on how the participatory process has been arranged. A visual discourse analysis studies this participatory design process arrangement as well as the interactivity of the medium on which an image is presented and the visual rhetoric, i.e. the combination of validity and readability, that is embedded in the images.

A synthesis of the analytical frameworks depicted in Chapters 3, 4 and 5 of this thesis (Figures 3.6, 4.1 and 5.3) constitutes the analytical framework of a critical visual research approach to studying the visual communication of landscape designs in participatory projects as meaning-making processes (Figure 6.1). These meaning-making processes are conceptualized as the relationship between semiotic complexity, interpretive habits, and visual discourse. The semiotic complexity of images gives rise to varying degrees of validity, readability and interactivity. What is considered to be valid, readable and interactive depends on the interpretive habits of the audience. Habits, in their turn, are influenced by the discursive power embedded in the images by the producers.

\section{$\Delta$}

\subsection{How visual meaning-making processes shape participatory projects}

The answer to the second part of the main research question of this thesis is derived from the application of the critical visual research approach to visual representations produced during the Rebuild by Design competition. Based on the empirical findings of Chapters 3, 4, and 5, the ability of visual representations' meaning-making processes to shape participatory projects can be categorized into a typology of nine 'semiotic qualities'. This typology is based on the premise presented in Chapter 3 of this thesis, i.e. design representations are signs that contain a great number of signs within themselves, and on the idea that each sign has a sign-function. The three main 'signs' of design representations constitute the 
Interpretive Habits

\section{Semiotic Complexity}

\section{Visual Discourse}

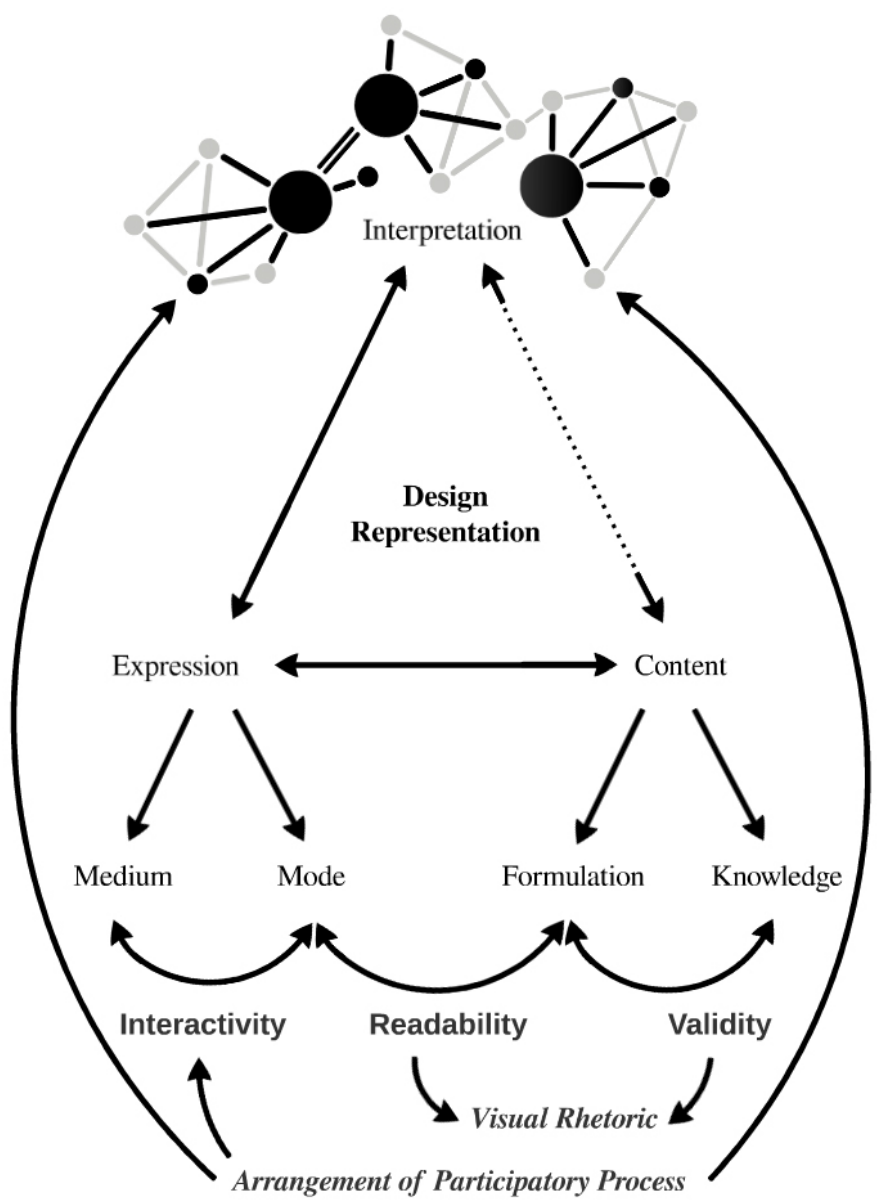

Figure 6.1. Analytical framework of a critical visual research approach to studying the meaningmaking processes of visual landscape design representations (own figure).

three main communicative elements of a visual landscape design representation: the validity of its content, the readability of its expression, and the interactivity of its medium (Figure 6.1).

As presented in the theoretical framework of Chapter 4, the sign-functions of each sign 'work' or signify by following three general lines of logic: iconic, symbolic, and indexical (Jappy 2013). Signifying logic is understood as the ways in which signs refer to their objects. For reasons explained in the next paragraph I 
translate the iconic and indexical signifying logic as representational and existential signification, respectively.

Iconic signification entails the relationship of physical or conceptual similarity of the sign to what it represents. This means that iconic signs are to be taken at facevalue: the depiction of a dike signifies the physical structure that needs to be built; the colour red signifies a certain degree of redness as a property of an object that is red; and an eye-level perspective signifies the perspective that is most commonly used by a viewer of average height. This definition deviates from the more common understanding of the icon as microcosm, i.e. a dominant representative symbol of a larger whole (Törrönen 2002). For example, this would be the case when the statue of liberty is seen as an icon of New York City. To avoid conceptual contamination, the iconic signifying logic in participatory processes is translated into a representational logic. Each communicational element of a design representation is representative of its actual degree of validity, readability, and interactivity.

Symbolic signification is based on subjective, cultural, and personal connotations. For example, the depiction of a dike can connote negative emotions connected to past flood events; strolling figurines might trigger memories of a past romantic relationship; a flock of birds could symbolize a sense of freedom. The communicative elements of design representations as symbolic signs in participatory processes are therefore not only representative of what they are; they are also symbolic for what they are perceived to be.

Indexical signification occurs through the logic of causality and by virtue of the occurrence and manifestation of a sign in a certain context. This means that the depiction of a dike in a landscape design refers to the flood hazards of the past that led to the urgency for implementing such an intervention. It also refers to the level of flood safety that could potentially be achieved in the future by constructing that dike. Indexes are also signs that enable or prevent certain activities from occurring. For example, the inclusion of figurines that show human activity, i.e. children playing or people strolling leisurely, are indexical for an area that is suitable for 
recreation. At the same time this type of human activity could make it difficult for certain ecosystems to thrive. As indexical signs within a participatory planning and design process, each communicative element is existential to the design project; they constitute the accumulation of previous iterations and determine possible pathways for the further development of a design.

In total, I define nine semiotic qualities at the intersection of the three communicative elements of design representations - validity, readability, and interactivity, and their three types of signifying logic - representational, symbolic, and existential sign-functions (Table 6.1).

Table 6.1. Nine combinations of communicative qualities and sign-functions.

\begin{tabular}{lccc}
\hline Communicative Element & \multicolumn{3}{c}{ Sign-Function } \\
\hline & Representational & Symbolic & Existential \\
Validity & $\mathrm{x}$ & $\mathrm{x}$ & $\mathrm{x}$ \\
Readability & $\mathrm{x}$ & $\mathrm{x}$ & $\mathrm{x}$ \\
Interactivity & $\mathrm{x}$ & $\mathrm{x}$ & $\mathrm{x}$ \\
\hline
\end{tabular}

Below, I define the nine semiotic qualities of Table 6.1 as a triadic typology of representational, symbolic and existential communicative elements that shape participatory planning and design processes. Nine drawings (Figures 6.2-6.4) represent hypothetical communicative settings within a participatory planning and design process. The figurines in these drawings depict a random number of hypothetical stakeholders that have particular interpretive habits. The square at the centre of the first six drawings (Figures 6.2-6.3) represents the same visual design representation with a different semiotic quality in play for each situation. In Figure 6.4 the lines stand for the progress of a planning and design process, which is hampered or accelerated by particular representational or symbolic qualities.

With each drawing I present a short definition of each semiotic quality and provide a contextualized example based on the empirical research documented in this thesis (Tables 6.2-6.4). Firstly, I distinguish between validity, readability, and interactivity in terms of their representational sign-functions (Figure 6.2 and Table 
6.2). Secondly, I distinguish between these same communicative elements in terms of their symbolic sign-functions (Figure 6.3 and Table 6.3). Lastly, I distinguish between these communicative elements in terms of their existential sign-functions (Figure 6.4 and Table 6.4).

The significance of each of the nine semiotic qualities for shaping participatory planning and design processes is addressed in the subsequent section following the typology presented below.

\section{A hierarchy of significance?}

The typology presented above distinguishes between the representational, symbolic and existential sign-functions of validity, readability, and interactivity. Each of these nine semiotic qualities of design representations is active whenever a design representation is produced or presented during participatory planning and design processes. However, not every semiotic quality has the same significance for every participant during a specific phase of the participatory planning and design process. For example, the existential function relates to precedent design choices and the consequences of these choices for the progress and implementation of a design. In the context of flood adaptation - due to the strict technical demands of flood defence designs - successful design implementation is particularly relevant. This makes existential functions more dominant during the planning and designing of multifunctional landscapes.

The existential qualities of design representations also lead to an accumulation of meaning during a participatory design process. This means that, during the course of a participatory design process, the existential qualities of design representations influence the formation of the interpretive habits of participants via the discursive power of their representational and symbolic qualities. Mapping the interpretive habits of the most relevant participants, during a specific phase of the design process, provides clues about which representational and symbolic qualities should be considered as most significant. For example, representational validity 
might be the most significant quality to convince a building engineer during the design of a multifunctional flood defence structure's technical construction plan. Symbolic validity, on the other hand, could be the most significant quality to convince potential investors of a design office's expertise.

Symbolic qualities might also serve as a catalyst for discussion or as an instrument for attracting media attention. Symbolic qualities are also key in creating design narratives where meanings are attached to design representations (and their content) that are not necessarily representational or existential. The 'appropriateness' of using symbolic qualities depends on how they will affect the evaluation of the design, the progress of the design process and consequently the chances of implementation. The likelihood that miscommunication occurs decreases by an awareness of the distinction between representational and symbolic qualities and by using the nine semiotic qualities consciously for a specific purpose. As a result, fewer participants are confronted with signs that are incompatible with their interpretive habits. Consequently, participants might be able to interpret the different 'critical functions' of design representations more easily and value their content accordingly. 


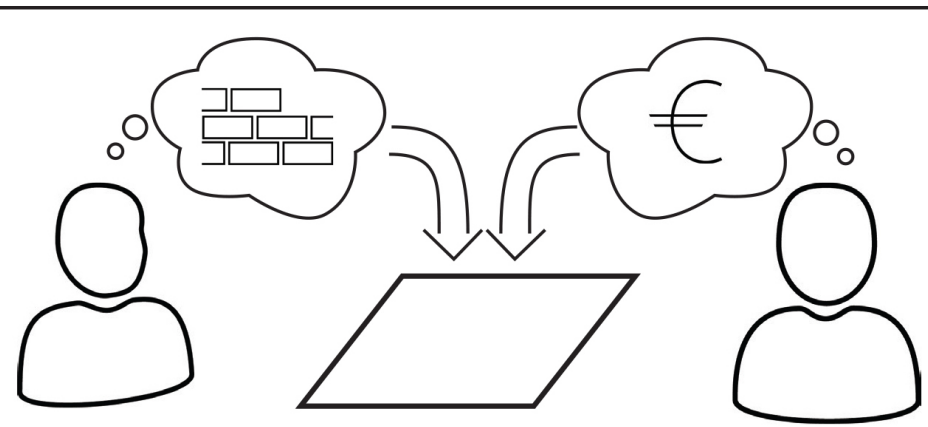

A. Representational validity: technical expertise about construction (left) and financial matters (right) are incorporated into the design.

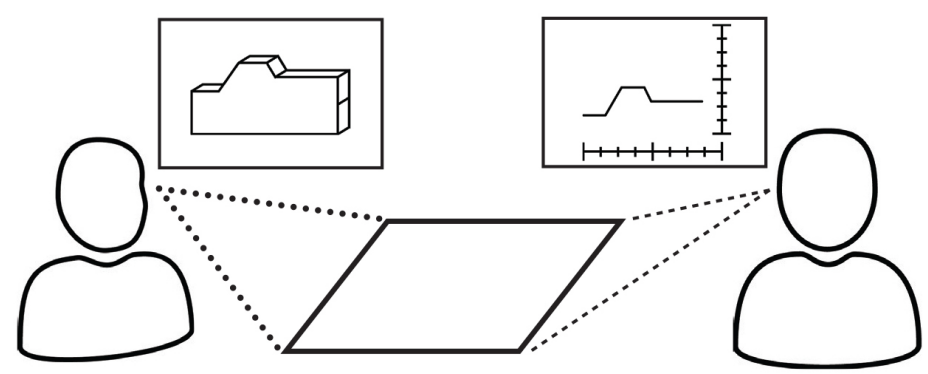

B. Representational readability: a 3D perspective view of a dike construction is needed (left), whilst another stakeholder requires a 2D cross-section with accurate measurements (right).

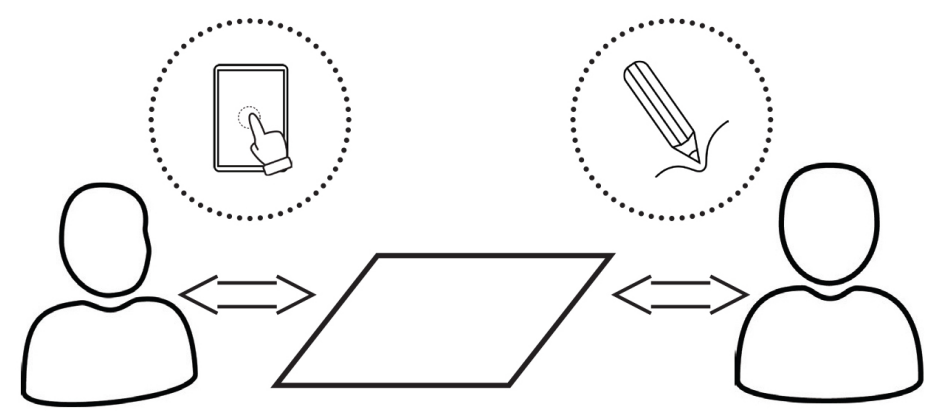

C. Representational interactivity: one stakeholder prefers a touch screen to zoom in and out of a map (left) whilst another stakeholder would like to be able to draw (right).

Figure 6.2. Representational semiotic qualities of design representations (own figure). 
Table 6.2. Definitions and empirical examples of representational validity, readability and interactivity.

\section{Representational qualities}

Representational validity (Figure 6.2A) relates to the extent to which the design's content represents the knowledge and preferences of the project participants. Not all participants' knowledge and preferences are included in the design in the same way and certain ideas are emphasized at the cost of others. Whether participants agree on the way that the different types of knowledge are integrated into the design depends on how that integration process occurred, and how well that process is reflected in the representation. For example, as concluded in Chapter 4, the New Meadowlands design proposal did not represent the knowledge of an environmental protectionist about natural conservation laws. From this participant's perspective the representational validity is low. From the designers' perspective the reason for this omission was that such legal restraints would hinder innovative thinking and have a different impact on the validity of the design.

Representational readability (Figure 6.2B) relates to the extent to which the knowledge and ideas contributed by participants can be read and understood by at least the participants themselves. The visual content analysis presented in Chapter 3 of this thesis shows the variability of graphical techniques, spatial projections and visual styles present in design proposals. The degree of readability refers to the extent to which these visual categories correspond to the visual language of their audience. For example, a civil engineer might require a cross-section representation to determine the height of a dike structure, whilst local inhabitants need an eye-level perspective impression to discern whether that height will have an impact on their view of the river. A combination of graphical techniques, e.g. map, artist impression, and schematic model, allows participants to recognize their own input in the design and understand how it relates to the input of others. As it was done in the Hoboken project (Chapter 5, Figure 5.6), such 'combined' visuals depict design solutions in different ways whilst being united by a shared visual rhetoric.

Representational interactivity (Figure 6.2C) relates to the extent to which the accessibility of the medium represents the way that participants are supposed to share their knowledge and perceive the design's content. Interactivity also relates to the ability of participants, as producers, to adapt the content and form of a design. From the perspective of the audience, interactivity also involves the degree to which participants are able to influence the way in which they perceive a design, e.g. by zooming in and out of an interactive map or by navigating a 3D model. However, drawing a sketch or reading a map are skills that not every participant necessarily possesses. Ensuring that the medium is in tune with the interactive skills of participants, or that participants are trained in these skills, is necessary for an inclusive participatory process. This could be done by organizing workshop sessions that facilitate the stakeholders' abilities to draw a sketch on paper, as it was done in the Living with the Bay project (see Chapter 5), or by constructing a scale model. 


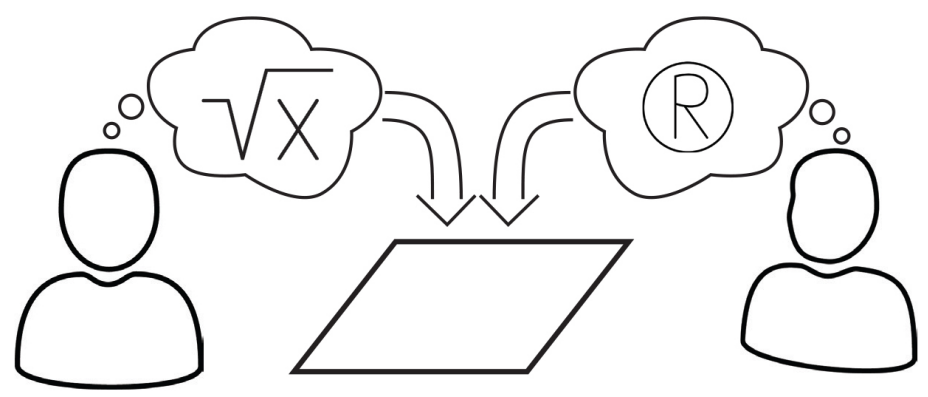

A. Symbolic validity: the suggestion of mathematical accuracy (left) or a token of authorship by a design firm (right).

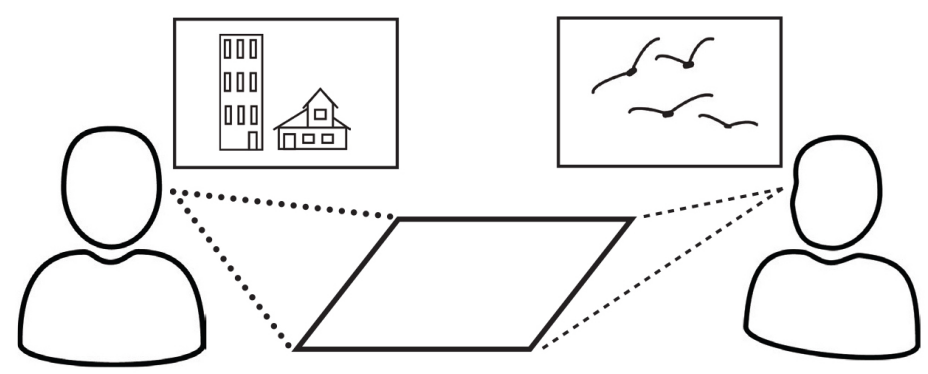

B. Symbolic readability: the depiction of expensive looking residential buildings (left) or the inclusion of a flock of birds to suggest natural qualities (right).

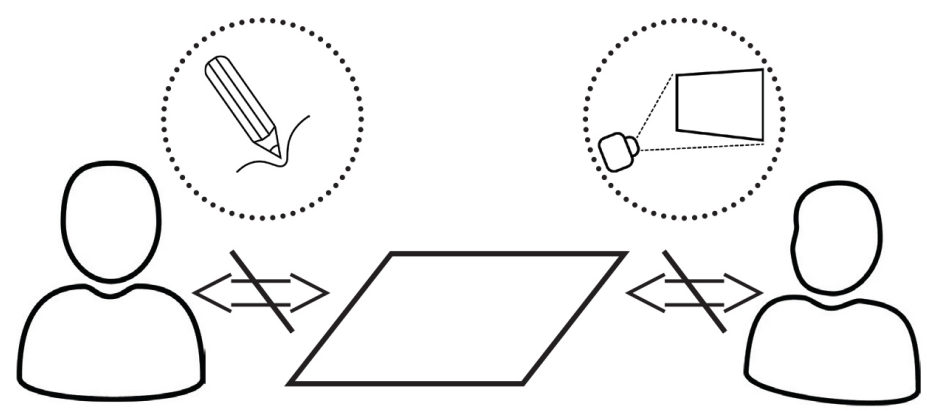

C. Symbolic interactivity: when stakeholders have the ability to draw on a finished design (left), or when a passive projector screen is used to present preliminary sketches (right).

Figure 6.3. Symbolic qualities of design representations (own figure). 
Table 6.3. Definitions and empirical examples of symbolic validity, readability and interactivity.

\section{Symbolic qualities}

Symbolic validity (Figure 6.3A) relates to the extent that participants perceive a design's validity in terms of its credibility and authority. Social and political authority influences the perception of a design's validity when, for example, respected institutions provide a design with their stamp of approval or when a design firm with the right credentials adds their token of authorship. Likewise, for certain participants, the inclusion of technical information with high mathematical credibility can add to the perceived validity of a design. For others, the quantification of highly subjective information, such as the social vulnerability map produced by MIT-CAU for the New Meadowlands project (see Chapter 4), might trigger a negative response and a lower perceived validity.

Symbolic readability (Figure 6.3B) relates to whether the style or visual rhetoric of an image connects to or is in conflict with the preferred interpretive habits of its audience. This type of semiotic quality is not about participants being able to discern the information presented, but about seeing the information presented in the way that a specific participant prefers or detests. Adhering to a specific visual frame or preference, such as making a photorealistic rendering of residential developments for a project developer, can result in a participant becoming more involved in a project. Likewise, it can also add visual meaning that is not necessarily part of the design's content. This 'visual surplus', e.g. a flock of birds or expensive looking residential buildings, can increase the visual appreciation of a design, but simultaneously create unrealistic expectations or trigger unnecessary resistance despite the high validity of design content. For example, in the New Meadowlands project (Chapter 4) some stakeholders described a rendering of waterfront residential development as an example of the visual language typically used by New York real estate developers, who they believe are attempting to colonize the New Jersey housing market.

Symbolic interactivity (Figure 6.3C) relates to whether the perceived accessibility of a medium corresponds to the degree of participation that stakeholders actually have. Specific phases of the design process require specific degrees of interactivity depending on how the participatory process is arranged. Presenting a Photoshop rendering on a projector screen during a first public meeting, as it was done in the New Meadowlands project, can be perceived as limiting participants' influence despite the fact that the intention is to just present some initial ideas (Chapter 4). Similarly, poster boards with different design scenarios on which participants can label their preferences can be perceived as open and interactive (Hoboken project, Chapter 5), despite the fact that the design ideas of each scenario have already been solidified into a proposal. In the first example, the perceived lack of interactivity could hamper the making of an inventory of local preferences. In the second example, the perceived interactivity can actually help to achieve the desired level of participation: the scenarios could be the result of co-design sessions and the posters serve as a way of getting feedback for selecting the most favourable scenario. 


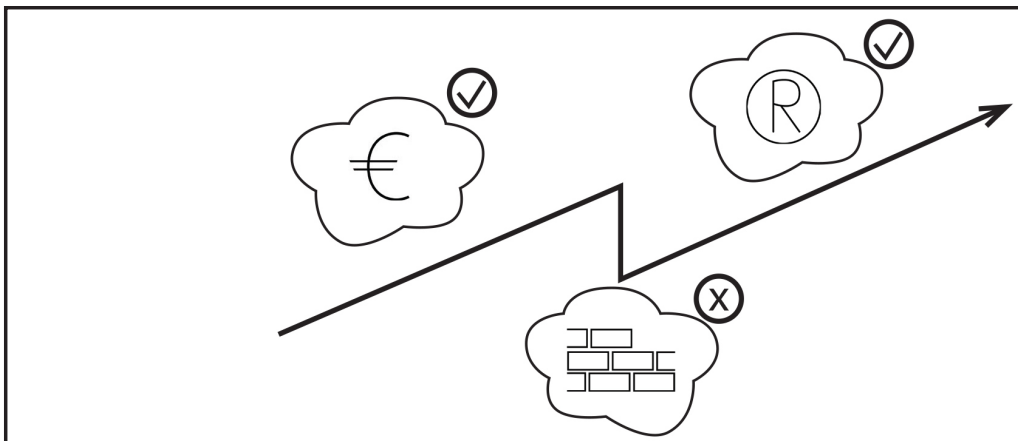

A. Existential validity: a positive project budget gave the green light to go ahead with the design (left), whilst technical problems with regards to construction slowed down the process (middle), which could eventually be solved by the approval of a renowned engineering firm which provided enough certainty to continue (right).

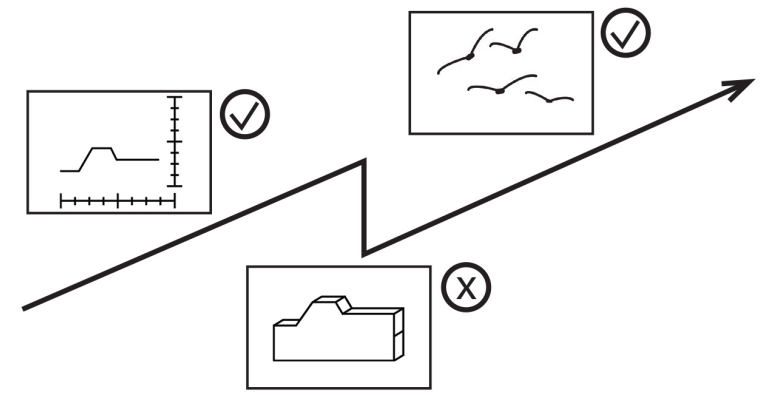

B. Existential readability: technical cross sections provided the right instructions to further work out the design (left). A 3D rendering of the dike structure confronted local stakeholders with its visual impact (middle), which temporarily halted the design process. An atmospheric impression was then used to secure public support for implementing the design (right).

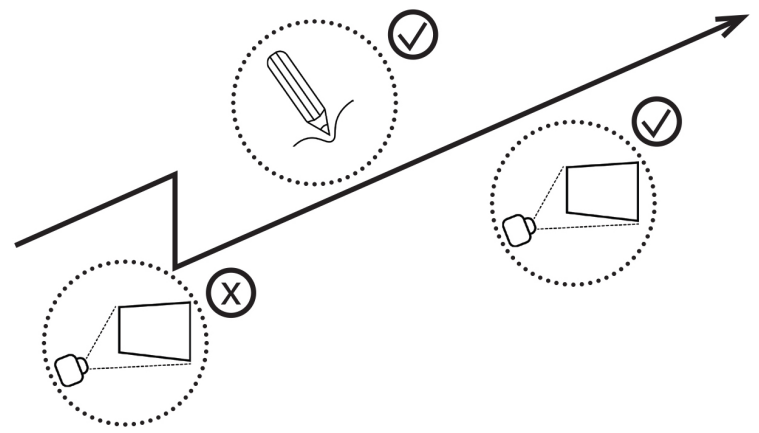

C. Existential interactivity: presenting designs at the beginning of a community meeting on a projector screen hampered public involvement (left), instead a drawing session helped to involve the relevant stakeholders (middle). That same projector screen was more suitable during a final presentation for the competition jury (right).

Figure 6.4. Existential qualities of design representations (own figure). 
Table 6.4. Definitions and empirical examples of existential validity, readability and interactivity.

\section{Existential qualities}

Existential validity (Figure 6.4A) relates to whether the validity of the design's content contributes to or detracts from the progress of the planning and design process. The design process can move forward if all or a majority of participants agree on the validity of design ideas, or if a design commissioner or competition jury is convinced that the proposed solutions will work. The most common qualities of a design, i.e. functionality, feasibility and visual aesthetics, are not considered equally valid in quality and importance by all participants. Finding the right balance between designers' and participants' preferences is crucial for the development of a design process. An environmental protection agency can threaten to take legal action and halt an entire design process, as it almost happened in the New Meadowlands project (see Chapter 4). Similarly, the approval of mayors and council members may be necessary for an 'institutional buy-in' to go forward into the implementation stage of the design process as was done in the Hoboken project (see Chapter 5).

Existential readability (Figure 6.4B) relates to whether the extent to which participants are able to read and understand the visualization contributes to or detracts from the progress of the planning and design process. Finding a shared 'visual language' that every participant understands or creating a 'tailor-made' representations for specific stakeholders, will ensure that the design ideas are understood in terms of their content, e.g. functionality, visual impact and/or feasibility. When the image is not understood 'properly' due to unreadability, i.e. a discrepancy in visual language between the producer and his or her audience, the quality of the design might not be evaluated based on its content but rather on a misunderstanding of that content. In the case of the New Meadowlands, for example, the design team made an effort to prevent 'unreadability' by combining different media and techniques in their poster presentation to cater to a wider range of audiences and by using the communicative setting of an 'open market' to provide additional explanations verbally (see Chapter 4).

Existential interactivity (Figure 6.4C) relates to whether the extent to which participants are able to engage with the medium of representation contributes to or detracts from the progress of the planning and design process. During the production of a design representation, the interactivity of the medium determines whether participants are able to interact with the content directly or indirectly. For example, participants who are willing to draw and are able to do so can share their ideas instantly, whilst a facilitated drawing session by a landscape architect, or a further working out of design ideas at the design office can lead to the additional mediation and translation of knowledge. A lack of interactivity can lead to the inability to share one's knowledge. For example, in the case of Rebuild by Design, such a lack of interactivity could have disqualified a project team from the design competition as the inventory of local knowledge through stakeholder workshops was a prerequisite activity. On the other hand, a sequence of workshops with interactive media could generate trust and engagement amongst participants and contribute to swift implementation. 


\section{$\nabla$}

\subsection{Discussion}

In this section, I will discuss both the process of abductive reasoning involved in developing a pragmatic conceptual framework and the critical visual research approach that has been developed in this thesis. I also discuss the relevance of the three main components of the critical visual research approach, i.e. semiotic complexity, interpretive habits and visual discourse, for studying landscape architectural design representations. Furthermore, I compare the typology of nine semiotic qualities, as distinguished in the previous section, to existing studies on design representations. This comparison will enable me to discuss in what way this typology and the developed research approach complement or contradict the existing body of knowledge. Finally, I will address the multimodality of design practice and its implications for design visualization.

\section{A pragmatic conceptual framework}

The development of the critical visual research approach presented in this thesis started out with the pragmatic hypothesis that Peirce's concept of the sign and his theory of 'triadic semiotics' provided a workable conceptual framework for studying how the meaning-making processes of visual landscape design representations shape participatory planning and design projects. This thesis shows that an open and abductive approach to the conceptualization of visual communication as a meaning-making process establishes a multi-perspective framework with which to study the different stages (Rose 2012) of visual communication. For example, drawing on the writings of Eco (1976), Peirce's model facilitates the further development of the triadic model with the dyadic model of Hjelmslev for the in-depth analysis of the semiotic complexity of the image in Chapter 3. The abductive nature of the resulting analytical framework lies in the combination and iterative translation of analytical categories based on 
literature from the fields of art history, film studies, environmental design and social semiotics through several rounds of coding.

The analytical framework of Chapter 4, which is used to analyse how the interpretations of visual representations are constructed by different project stakeholders, is the result of a similar abductive process. Peirce's theory of the Interpretant allows for a under-explored connection with post-foundational discourse theory via the writings of Gaspard $(2015,2016)$ and Marttila (2016). A comparison of both theories revealed the strong compatibility of concepts. The application of the resulting combined analytical framework to the Rebuild by Design projects demonstrates the analytical power of an explicitly social semiotic approach to discourse analysis for uncovering relevant connections that might otherwise be ignored.

The inclusion of Barthes' writings on the rhetoric of the image (Barthes 1977) and Foucault's power/knowledge and governmentality concepts (Foucault 1980, 2000) also proves to be an useful addition for studying the discursive power and influence of the image producer on the interpretations of images in Chapter 5. Barthes' theory is quite analytically explicit in terms of analysing elements of visual rhetoric, whereas the operationalization of the Foucaultian concepts required several more abductive steps. In this case the interview material was explored for possible translations of the 'technologies' and 'apparatuses' of the designer in terms of medium interactivity and the arrangement of the participatory process before settling on the final definitions. Finally, in the concluding chapter of this thesis, Peirce's typology of sign-functions (Jappy 2013) contributes to the final frame with which the different ways in which the validity, readability and interactivity of visual representations shape participatory planning and design processes can be categorized.

\section{A critical visual research approach}

The study presented in this thesis adds several new perspectives to and insights into the visual communication of landscape design representations. Previous 
research into visual research approaches and frameworks of evaluation criteria for design visuals (Bates-Brkljac 2009; Downes \& Lange 2015; Sheppard 2001) have led to the dominant circulation of such criteria as visual 'realism', 'accuracy' and 'abstraction'. Definitions of these terms vary between authors and most critical studies on the use of visualizations in landscape design practice target specific digital and hyper-real visualization technologies. In this thesis, visual design representations are conceptualized in terms of their semiotic qualities, i.e. what these images could mean and what meaning-making processes they are part of. Representations are not conceptualized as merely 'images' or 'tools'; they constitute an interplay of design content, visual expression, their moment of production, and the context of their interpretation. In light of this re-conceptualization existing criteria for design visualization become highly situational. What is considered to be 'realistic', 'accurate' or 'abstract' is very much dependent on the nature of the project, the type of participation, the phase of the design process and the type of stakeholder involved.

The combination of the critical visual research approach and the analytical framework presented in this thesis provides a strong tool for dealing with this situational complexity. For example, this thesis introduces a semiotic vocabulary that exceeds the categorization of content or visual techniques. Rather, it promotes the idea that each colour, perspective, textual element, drawing technique or medium is a sign in itself. As signs, each of these variables influences the interpretation of the image as a whole and, therefore, the interpretation of the design content which is represented. The overlooking of this semiotic complexity is apparent, for example, in past experiments that aim to research the effectiveness of different 2D, 3D and VR techniques. In these studies (e.g. Bates-Brkljac 2009; Gill et al. 2013), prototypes are produced with different graphical techniques, yet not all remaining variables remain the same: additional colours, textures, and shading are introduced in the different prototypes that detract from the variable under scrutiny. This thesis offers an analytical framework which can enable the 
identification of these additional signs more clearly and, consequently, to take them more consciously into account while conducting visual experiments in both controlled and practical environments.

Moreover, in this thesis explicit attention is paid to the process of interpretation and the socio-political context that influences the 'interpretive habits' of project stakeholders. Although the relevance of taking into account the audience of design representations has been widely acknowledged (e.g. Lovett et al. 2015; Williams et al. 2007), the audience's interpretations are rarely conceptualized as interpretive habits involving of discursive networks that can be triggered or activated by the specific signs or sign systems that make up the design image. In establishing such a strong connection between the semiotic complexity of images and the sociopolitical context of the planning and design process, interpretations are no longer considered a black box of unpredictable responses. Instead, they consist of a contingent range of possibilities which can be anticipated and acted upon by the producers of design representations.

Finally, the discursive power of design images and their production process has often been overlooked, despite some critical studies of hyper-real images of urban and landscape designs. These studies (e.g. Kullmann 2014; Lee \& Pae 2017) justifiably identify the discrepancy between representations and their built counterparts and recognize the potential for and danger of miscommunication. However, from the discursive viewpoint presented in this thesis it is possible to better conceptualize and explain the influence that such imagery has on the interpretive habits of its audience. Visual discourse, by its conceptual nature, struggles for assimilation and domination (Rose 2012) within the discursive networks that make up the socio-political context of participatory planning and design processes. In recognizing such discursive powers, e.g. by looking at medium interactivity and visual rhetoric, the influence of planners and designers on the interpretations of their representations can be identified more clearly and used more consciously. 


\section{A typology of semiotic qualities}

The comprehensiveness of the typology of nine semiotic qualities becomes clear when it is compared to existing literature on the use of landscape visualizations. Most previous research has focused on specific types of images, e.g. maps, interactive tools, or photomontages. These techniques are assessed according to a singular perspective, i.e. either purely on their validity, readability, or interactivity but not in their entirety. For example, interactive 3D landscape visualizations are a commonly researched visual technique in terms of interactive knowledge construction in participatory planning processes (e.g. Bailey \& Grossardt 2010; Gill et al. 2013; Schroth et al. 2011). The study of Schroth et al. is focused on the integration of conceptual and empirical knowledge across disciplines and local community stakeholders, wherein interactivity is used to increase the mutual understanding of complexity data by enabling a dialogue between participants. Existential interactivity, in the terms of this thesis, is seen in that study as a means of establishing representational validity, i.e. whether all knowledge is properly integrated in the service of existential validity. The symbolic dimensions of interactivity, e.g. the 'wow effect' of the ability to fly through virtual environments using different perspectives and its effect on visual preference, is not addressed, neither is the influence of different forms of readability such as colour schemes and 3D realism, for example.

Other studies deal with usability in terms of user experience of new media and techniques in general, but not necessarily in relation to planning and design processes (e.g. de Boer et al. 2011; Jallouli \& Moreau 2009; Ruotolo et al. 2013). For example, de Boer et al. describe techniques for the realistic visualization of historical landscapes. Accurate data needed for ensuring content validity often does not exist for the historical reconstruction of landscapes. Semiotically, the signs that are created, i.e. visual representations, have no real object. Rather, the image signifies a preconceived notion of what historical landscapes are or could have looked like. As a result, the study focuses on the representational interactivity of $3 \mathrm{D}$ environments 
and the symbolic readability of historical content, not taking into account how that affects the users' perceptions of that content, i.e. its representational validity.

Studies of affective appraisal (e.g. Houtkamp 2012; van Lammeren et al. 2010) approach the comprehensiveness of the representational and symbolic qualities the most. These studies deal with symbolic readability, for example, by studying the emotive responses of audiences to modified visual traits (textures, figurines) of design representations. These visual traits are placed in relation to representational validity, i.e. they provoke value statements about the physical environment represented. This validity and readability is also combined with multisensory interactivity, for example, by inducing environmental sounds into the experiments. However, the critical counterpart of such - predominantly technical - studies of visualizations, that is the symbolic dimensions of validity and interactivity, is not taken into consideration.

Out of the range of visual techniques under scrutiny, analytical design images such as maps are by far the most analysed and critically studied. However, when maps are discussed critically, the focus lies on the symbolic validity, e.g. the credibility and authority of maps and their authors (Wood 2003), and symbolic readability, e.g. the visual rhetoric used to achieve a desired effect (Crampton 2001; Dühr 2007; Monmonier 1996). These semiotic qualities are often discussed in the light of their existential effects: how they influence decision making processes (Carton 2007; Gaberell \& Debarbieux 2014) and/or the public debate (Bueno Lacy \& Van Houtum 2015). When maps are discussed technically, e.g. by GIS experts, representational validity is primarily addressed in terms of how accurately spatial data is classified, aggregated, and simplified. These studies lean on classic works (e.g. Bertin 2011; Tufte 1990) in terms of representational readability, i.e. how spatial data can be represented most accurately. Most notably, the semiotic qualities of interactivity are only discussed with regards to technological innovations such as the map table (Vonk \& Ligtenberg 2010). Although such studies centre on the representational interactivity of the medium and its effects on the validity 
of content, its effect on the readability of maps is often not discussed or only in general terms.

Techniques that are used to produce conceptual images, such as photomontages and 2D/3D artist impressions, are discussed critically with regard to their hyperreal qualities (e.g. Kullmann 2014; Lee \& Pae 2017). These discussions on hyperreality focus on symbolic readability and its existential implications and its relationship to representational validity. Interactivity is not addressed critically, even though the detailed and 'finished' nature of these 'artistic' landscape representations - often presented on passive media such as projector screens, websites, and billboards - signifies a degree of certainty that does not cohere with the design's conceptual and 'open' character.

Implementational images, especially in relation to technical designs such as flood defence structures, centre on representational readability and validity. These images, e.g. maps, cross sections, and blue-prints, are discussed as technical representations with a specific purpose: to explain how a design works and how it needs to be built (e.g. van Veelen et al. 2015; Voorendt 2017). Despite the clarity of functional information provided by such images, these studies generally neglect the (unintended) symbolic qualities and the potential effects of technical drawings and mathematical formulas on the progress of a design project.

To conclude, even though the literature discussed above contains clear analytical foci, several factors (e.g. colours, contrasts, perspectives, techniques, media) unintentionally contaminate the results of these visual experimental studies. Of course, it is difficult (if not impossible) to conduct visualization experiments in an environment that is sufficiently controlled to enable the study of all nine semiotic qualities in play. Yet, an awareness of a broader range of semiotic qualities - as promoted in this thesis - could help neutralize such factors and achieve more controlled research environments than are currently being used. 


\section{Participatory planning and design: a situated practice}

The critical visual research approach and analytical framework presented in this thesis can be used to study all the types of visual imagery that are used during various phases of design processes. This comprehensiveness is necessary when dealing with a diversity of stakeholders and is reflected in the diversity of validity, readability and interactivity of design representations. These three communicative elements and their representational, symbolic, and existential sign-functions may complement each other, but they can equally well overpower or even contradict each other. The balance and outcome will vary from project to project. For example, the validity of the content that is being represented will depend on the interpreter: a civil engineer will consider the feasibility of the project based on mathematical calculations; a designer may appreciate a project for its visual aesthetics; local inhabitants may worry about the sunset being hidden by a dike. All of these values contain a certain validity, which will influence how the design is interpreted. The readability of representations will also depend on the stakeholder that views them. For example, participants who are intimately involved with the project might understand a design without actually 'reading' it because they know the content by heart. Similarly, the outside jury of a design competition - without such involvement - will need to interpret the design's content purely on its visual and interactive merits. Finally, visual techniques are not equally interactive, and can be created and interpreted differently. GIS maps can be overlaid with hand drawn sketches; photomontages can be created using photos made by local inhabitants and 3D models can be explored at leisure with online gaming engines.

Representational, symbolic, and existential sign-functions seem to provide an encompassing categorization for studying the qualities of design representations that shape participatory planning and design processes. However, semiotically, the symbolic sign-function is by far the broadest and most complex category of signfunctions. For example, in a design competition the designer or design team must meet the standards set by a panel of experts who often apply a range of 'fuzzy' 
criteria (van Wezemael 2011), rather than the standards set by a single client. Competitions, by their very nature, consist of arenas where a diversity of discourse and interests from different fields intersect according to a prescribed format. Design briefs and their competition submissions could, therefore, lead to results that 'reflect the preferences of jurors rather than clients or users and can be derailed by political intervention' (Davison et al. 2018: 2). Persuasion and manipulation are sometimes regarded as valuable tactics in attaining public and political support for design ideas in competitive settings. These visualization strategies take place in the context of the symbolic qualities of the typology presented in this thesis and entail, amongst other things, the use of narrative structures and visual rhetoric. Although the visual rhetoric of design representations is discussed in this thesis, a more intricate analytical framework is necessary to study different rhetorical strategies, their visual tactics, and their intended and unintended consequences for planning and design processes. A Peircean approach of speculative rhetoric to perlocution (Gaspard 2018), i.e. the myriad effects of a speech act on its audience, could help to establish a clearer visual code of conduct for typical communicative settings during planning and design processes.

Furthermore, following the line of thinking of many continental philosophers (Baudrillard 1981; Lacan 1977; Nietzsche 1967) the 'symbolic order' is becoming a more dominant sign-function, trumping its representational consort in terms of significance. This would entail that, in some planning and design processes, one could achieve great success by focusing on symbolic rather than representational validity, readability and interactivity. As a result, credibility, authority, the suggestion of accuracy, visual seduction and a facade of open participation could become leading as opposed to creative problem-solving, visual clarity and democratic co-design workshops. Such a scenario is becoming more likely as scholars (e.g. Chomsky \& Barsamian 2017; Žižek 2015) recently discussed the arrival of the 'post-truth' era in the light of 'fake news' where facts are deemed secondary to personal opinions. In this context questions arise relating to visualizing designs 
in a way that is expected by both commissioners and the public while maintaining integrity as a landscape designer. Within this context of seemingly incompatible demands a further specification of the three symbolic sign-functions presented in this thesis, drawing on the fields of psychology, cognitive semiotics, and cultural studies in general, could provide an even clearer perspective on the most or least significant symbolic qualities of design images.

\section{$\triangle$}

\subsection{Reflection on the research approach}

In the following section I reflect on the conceptual framework of this thesis and its possible limitations, as well as on my choice of research design, the validity of the case study, and my position as a researcher in relation to the subjects of inquiry.

The conceptual framework that I use in this thesis serves as a theoretical model and should be considered to be adaptable to change and reconceptualization provided that epistemological consistency is maintained. This means that the theory of Peircean semiotics provides a communication model that is applicable to any communicative context, as long as the various sign components are reconceptualised appropriately. Peirce's triadic model of the sign provided me with a clear structure for studying representations with three distinct research foci: the image, its interpretation, and its production. By applying this model to one and the same case study the differences between and complementarity of the three foci was made clear and the relative validity and purpose of each focus area was demonstrated within the same planning and design context.

The choice for the Rebuild by Design $(\mathrm{RbD})$ competition as a case study is based on its focus on multifunctional flood defence (MFFD) landscapes and the nature of its participatory planning and design processes. Studying design competitions has advantages in terms of the internal validity of the research, e.g. a wealth of documentation on comparable projects, yet in terms of external validity does 
not always provide an accurate representation of planning and design practice (Banerjee \& Loukaitou-Sideris 1990). In the case of $\mathrm{RbD}$, several respondents noted that the explicitly participatory co-design approach advocated in the RbD design brief did mirror approaches commonly used in planning and design practice. However, the same respondents also noted that the financial and time restraints that were caused by the competition framework led to 'pressure cooker' situations where there could not be a follow up to every stakeholder meeting. Even though the planning and design processes that took place as a whole are not seen as representative of planning and design practice, the communicational settings used and the visual communication processes that occurred between designers and stakeholders are nevertheless considered representative of the way communication takes place in planning and design practice.

By studying these visual communication processes in terms of interpretive habits and visual discourse, 'meaning' itself is not the main research object, but rather the process of meaning-making. To conduct a valid study of how representations are interpreted differently by different project stakeholders, a detailed account of different interpretations of the same project and project context was needed (Vannini 2007). Considering the international character of the case study and the resulting availability of respondents, 20 interviewees - both audiences and 'producers' - were identified based on their professional background as key stakeholders within the different $\mathrm{RbD}$ project proposals. This group provided a varied yet detailed enough account of the communication processes that occurred during the design competition. This allowed me to both demonstrate the critical visual research approach developed in this thesis and provide examples of the topology of the nine semiotic qualities presented in this chapter.

Semiotic analysis, central to this thesis, constitutes a form of interpretive research (Yanow \& Schwartz-Shea 2014). Since interpretive research is characterized as being an open, abductive approach without fixed analytical structures, the positivist criteria of reproducibility and generalizability are less relevant (Schwartz-Shea 
\& Yanow 2012; Silverman 2001). Rather, the validity of my interpretations as a researcher depends on my positionality in relation to the subjects of my inquiry, and to the transparency, systematicity, and imitability of my research approach and data analysis. The 'double hermeneutics' concept is often used for positioning the researcher in relation to his or her research subjects (Alvesson \& Sköldberg 2000; Marttila 2016). This concept entails a transparent description of the interpretations of a research phenomenon by interviewees, respondents, and literary sources $\left(1^{\text {st }}\right.$ order hermeneutics), and the effects of the interpretive framework of the researcher ( $2^{\text {nd }}$ order hermeneutics).

In this thesis I take up three different positions in relation to empirical data in three different chapters. Firstly, in Chapter 3, that of the iconographer who studies the semiotic complexity of design images and their contextual information through a multi-layered interpretive iconographic framework. As iconographic interpreter, I only studied the so-called representational and iconographical layers of the meaning of design representations. I intentionally left out the third iconological layer of iconographical interpretation since this layer constitutes the most personal and connotative interpretation of images. Secondly, as a situational analyst, in Chapter 4 I studied interpretive habits of project stakeholders by analysing interviews from a social semiotic perspective. Here, my position was to find semiotic connections, following indexical, iconic and symbolic sign functions, between and within the different descriptions of interviewees. The anonymity of the interviewees has been maintained at their request and personal opinions that were not meant to go on record were excluded from the transcriptions. Thirdly, as a visual discourse analysist, in Chapter 5 I studied the visual rhetoric of images and their visual discourse through interview- and document analysis. The analysis of the visual rhetoric of the design images constitutes my interpretation of images whilst following the Barthesian theory on the rhetoric of the image. I describe and explain this 
interpretive process of 'de-naturalising' the image in an open, transparent way to explain what the visual rhetoric of the analysed images could consist of.

\section{$\Delta$}

\subsection{Suggestions for further research}

Design representations have to function within different socio-political contexts during the implementation, construction and (ideally) monitoring of a design. The final phases of the planning and design process were not addressed by the empirical situations analysed in this thesis. The outcomes of the $\mathrm{RbD}$ competition consist of design concepts rather than comprehensive master plans or technical designs fit for implementation. This means that the $\mathrm{RbD}$ case study does not represent the entire process of designing, even though the comprehensive participatory strategy of $\mathrm{RbD}$ has led to communication processes that are representative of design practice. For future research, the scope of a critical visual research approach to participatory designs and their representations could be widened to include the final stages of the planning and design process. A study and monitoring of the entire planning and design process could answer the more specific question of how visual design representations influence the extent to which a design actually gets built in the way that was intended.

Chapter 2 of this thesis proposes studying planning and design processes using the theory of Simulacra and Simulation (Baudrillard 1981). This would entail tracing the different stages of 'simulacra' that a design goes through and would require a meticulous reconstruction of the planning and design process or an active engagement with that process by the researcher. Both approaches are beyond the scope of this thesis, yet future research could benefit from a more action-research oriented strategy to advance the critical visual research approach proposed in this thesis to a more transformative approach. Such a more transformative actionresearch strategy could help to further verify and enrich the semiotic vocabulary 
of Chapter 3, study the degree to which interpretations can be anticipated using the SSPDA method presented in Chapter 4, and study the discursive power of representations, as proposed in Chapter 5 in more detail through visualization experiments during the course of a planning and design process.

Apart from the participatory planning and design processes of MFFD projects many cases could benefit from the critical visual research approach presented in this thesis. For example, the visual content analysis of Chapter 3 has been applied to a study of the use of photographic comparison to triangulate qualitative outdoor thermal perception surveys (Cortesão et al. 2018). This study suggests that the identification of particular combinations of visual signs, which signify degrees of outdoor thermal comfort, can help (urban) designers to conduct more valid qualitative inquiries and, in turn, communicate the microclimatic conditions of their designs more effectively. Similarly, participatory and non-participatory planning and design projects in general, with themes that range from the energy transition and urban microclimate to heritage preservation, can benefit from studies of design preferences based on visual impact.

\section{$\triangle$}

\subsection{Societal relevance}

The conclusions of this thesis provide insights into visual communication processes that have important implications for planning and design practice. However, considering the diversity and complexity of multifunctional landscape projects, this thesis cannot deliver clear-cut recommendations for the use of visual representations in participatory design processes. Rather, it suggests ways of looking at visual representations and organizing the planning and design process that can help planners and designers, as process facilitators, to determine the most appropriate communicative strategy needed at a specific moment, for specific participants. Making 'appropriate' visual representations requires both the 
ability to look critically at the design's content, as well as the ability to express that content in a visual way while taking into account the creative and interpretive context of a participatory design process. This means one needs to be sensitive to participants' backgrounds, i.e. both their personal and professional interpretive habits, understand how visual techniques function discursively and assess what degree of semiotic complexity is appropriate in a given context.

The ability of a planner or designer to facilitate communication between experts, stakeholders, and local participants influences the extent to which scientific, expert and local knowledge is integrated and political and public support is attained. Such capacities are not gained overnight and will require practical experience with participatory planning and design projects and a stronger emphasis on these abilities in design education. Nevertheless, based on the insights of this thesis, the following strategies are suggested for planners and designers who facilitate participatory processes. For a visual representation to be effective and communicate its content successfully, landscape planners and designers need to pay close attention to the three main communicative elements of visual design representations: validity, readability and interactivity.

Firstly, the validity of design representations can be improved by ensuring that the design content depicted is considered to be feasible and informative. Planners and designers can increase the feasibility of a design's content by preventing the inclusion of visual signs that could, logically speaking, not be present at the location where they are depicted. These signs can be small and relatively harmless for the overall interpretation of the image, e.g. jogging figurines in tall grass or a flock of birds in a crowded city street. However, bigger (and therefore more influential on the interpretation and evaluation of the design) are signs that signify a landscape function that could never exist, e.g. the depiction of high-rise residential development at locations that are currently characterized by single family homes.

Design representations can be made more informative by making sure that the functional claims made by the designers are supported by the visual signs in 
the image. The planner or designer needs to check whether all visual elements are grounded in the content of the design, or whether they are only there for visual beautification or so-called 'window dressing'. A proper grounding of signs with design content prevents possible over-interpretation. Such over-interpretations occur when certain functions or qualities are inferred that are not supposed to be part of the design, or when emotional connotations are made that will probably not be experienced in the implemented design.

Secondly, the planner or designer can further help to prevent over-interpretation by taking into account the degree of readability, i.e. whether the viewer can read and understand the content that is represented. Questions that help to ascertain whether this is the case relate to the extent to which the image shows how the design 'works', what problems it aims to solve and the extent to which it should be considered a 'sketchy' or 'finished' design. Furthermore, it is important for planners and designers to have a good understanding of the visual literacy of their audience. This means that image producers should know how familiar local inhabitants are with cross-sectional drawings or whether civil engineers will discern useful information from beautified Photoshop renderings. Alternatively, it could also be worthwhile to develop a shared visual language with the members of a consistent stakeholder group. However, the involvement of external audiences, e.g. politicians or a competition jury, requires a careful translation of the developed design into a readable image for that new audience at a different design phase.

Thirdly, this thesis shows that the degree of interactivity that a particular medium offered during a participatory planning and design process also consciously or unconsciously signifies the level of participation that is desired by the process facilitator. This means that the designer, planner, and/or process facilitator needs to determine the desired level of interactivity for a particular design representation in a particular communicative setting. They must ask themselves how much control is needed over the integrity of the scene, e.g. the perspective and duration of viewing or over the integrity of the content, e.g. the ability of participants to add 
or delete ideas. Another question is whether the type of interactivity should be a quality in itself, e.g. the 'wow-effect' of virtual reality glasses; or to what extent the image should be self-explanatory, e.g. whether there is a possibility for providing additional information.

The development of a necessary sensitivity to the complexity of stakeholder groups and its consequences for visualization strategies can start in the classrooms of landscape planning and design education. Courses on design representation can, besides focusing on technical skills, also teach students about the use of visual signs and how they might be interpreted by different audiences. A visualization lab where students can experiment with different techniques and media (e.g. virtual reality, 360 degrees photomontages, 3D printing) could help them find out how usable and useful such techniques are for designing and for communicating and evaluating their ideas. Furthermore, a stronger link between landscape architecture courses and strategic spatial planning could provide a more encompassing framework for taking into account the socio-political dimension of landscape design, a dimension often overlooked from within the perspective of the design studio.

By acknowledging the complexity of participatory planning and design processes and by producing (and interpreting) visual representations according to the critical visual research approach developed in this thesis, communication can be more conscious, empathic and, ultimately, more effective. This can lead to an increased sense of confidence and design ownership among the project stakeholders and participants which in turn will improve the chance that the design will be implemented as intended.

Finally, a key insight based on this thesis research is that planning and design projects cannot be reduced to a single image: a 3D bird's eye view does not show all the design ideas that make up a project; neither does a handmade scale model. The only way of achieving a 'complete' representation of a design project - if such a thing were possible - would be to look at all of the images that were produced and to identify the various ideas and interests that are embedded within them 
much in the same way that has been done in the research approach presented in this thesis. This also means that the hyper-real 3D renderings or photomontages that end up on a website or billboard only represent a small part of a design, even though these are often the images that become the focus of public discussion. 



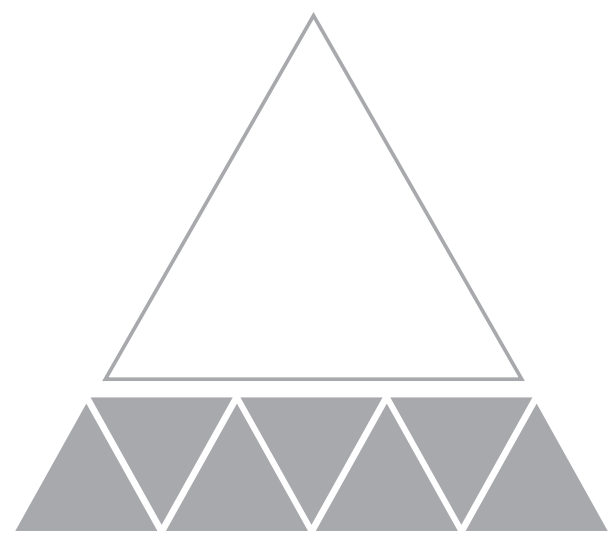





\section{References}

Acton, M. (1997). Learning to Look at Paintings, London: Routledge.

Allwood, J. (1981). On the Distinctions Between Semantics and Pragmatics. In W. Klein \& W. Levelt, eds., Crossing the Boundaries in Linguistics, Dordrecht: Reidel, pp. 177-189.

Altamrano, M., \& van der Heijden, J. (2013). Water keren en combineren: functiecombinaties bij waterkeringen, Delft.

Altürk, E. (2008). Architectural representation as a medium of critical agencies. The Journal of Architecture, 13(2), 133-152.

Alvesson, M., \& Sköldberg, K. (2000). Reflexive methodology: new vistas for qualitative research, London [etc.]: SAGE.

Amoroso, N. (2012). Representing Landscapes: A Visual Collection of Landscape Architectural Drawings, London: Taylor \& Francis.

Anvarifar, F., Zevenbergen, C., Thissen, W., \& Islam, T. (2016). Understanding flexibility for multifunctional flood defences: a conceptual framework. Journal of Water and Climate Change, 7(3), 467-484.

Appleton, K., \& Lovett, A. (2005). GIS-based visualisation of development proposals: Reactions from planning and related professionals. Computers, Environment and Urban Systems, 29(3), 321-339.

Bafna, S. (2008). How architectural drawings work - and what that implies for the role of representation in architecture. The Journal of Architecture, 13(5), 535-564.

Bailey, K., \& Grossardt, T. (2010). Toward Structured Public Involvement: Justice, Geography and Collaborative Geospatial/Geovisual Decision Support Systems. Annals of the Association of American Geographers, 100(1), 57-86.

Balmori, D. (2014). Drawing and Reinventing Landscape, Chicester: John Wiley and sons.

Banerjee, T., \& Loukaitou-Sideris, A. (1990). Competitions as a Design Method: an Inquiry. Journal of Architectural and Planning Research, 7(2), 114-131.

Barnaud, C., Le Page, C., Dumrongrojwatthana, P., \& Trébuil, G. (2013). Spatial representations are not neutral: Lessons from a participatory agent-based modelling process in a land-use conflict. Environmental Modelling and Software, $45,150-159$.

Barnett, R. (2013). Emergence in landscape architecture, London: Routledge.

Barthes, R. (1977). Rhetoric of the Image. In S. Heath, eds., Image, Music, Text, London: Fontana Press, pp. 32-51.

Barthes, R. (2009). Mythologies, London: Vintage Classics.

Bates-Brkljac, N. (2009). Assessing perceived credibility of traditional and computer generated architectural representations. Design Studies, 30(4), 415-437. 
Baudrillard, J. (1981). Simulacra and Simulation, Ann Arbor, MI: University of Michigan Press.

Baudrillard, J. (1988). Selected writings, Cambridge: Polity Press.

Baugh, B. (1997). Making the difference: Deleuze's difference and Derrida's différance. Social Semiotics, 7(2), 127-146.

Bell, P. (2001). Content analysis of visual images. In T. Van Leeuwen \& C. Jewitt, eds., Handbook of Visual Analysis, London [etc.]: SAGE, pp. 10-34.

Bellentani, F. (2016). Landscape as Text. In C. J. R. Higuera \& T. J. Bennett, eds., Concepts for Semiotics, Tartu: University of Tartu Press, pp. 76-87.

Bergman, M. (2009). Peirce's Philosophy of Communication, London [etc.]: Continuum International Publishing Group.

Bernard, H. R. (2011). Research methods in anthropology?: qualitative and quantitative approaches, Lanham, MD [etc.]: AltaMira Press.

Bertin, J. (2011). Semiology of Graphics: Diagrams, Networks, Maps, Redlands, US: ESRI Press.

Biddulph, M. (2014). Drawing and Thinking: Representing Place in the Practice of Place-making. Journal of Urban Design, 19(3), 278-297.

Bishop, I. D., \& Lange, E. (2005). Visualization in landscape and environmental planning: Technology and applications, London: Taylor \& Francis.

Bishop, I. D., Pettit, C. J., Sheth, F., \& Sharma, S. (2013). Evaluation of data visualisation options for land-use policy and decision making in response to climate change. Environment and Planning B: Planning and Design, 40(2), 213233.

Bonnett, A. (2014). Unruly places: Lost spaces, secret cities and other inscrutable geographies, Toronto: Penguin.

Brown, M. (2010). Nicaraguan invasion? Blame google maps. Retrieved December 1, 2017, from https://www.wired.com/2010/11/google-maps-error-blamedfor-nicaraguan-invasion/

Bryman, A. (2012). Social Research Methods, Oxford: Oxford University Press.

Buchanan, R. (1992). Wicked Problems in Design Thinking. Design Issues, 8(2), 5-21.

Bueno Lacy, R., \& Van Houtum, H. (2015). Lies, Damned Lies \& Maps: The EU's Cartopolitical Invention of Europe. Journal of Contemporary European Studies, 23(4), 477-499.

Bushell, S. (2012). The Slipperiness of Literary Maps: Critical Cartography and Literary Cartography. Cartographica: The International Journal for Geographic Information and Geovisualization, 47(3), 149-160.

Carton, L. J. (2007). Map making and map use in a multi-actor context: Spatial visualizations and frame conflicts in regional policymaking in the Netherlands, Delft University of Technology, NL.

Cederström, C., \& Spicer, A. (2013). Discourse of the real kind: A post-foundational approach to organizational discourse analysis. Organization, 21(2), 178-205. 
Chandler, D. (2007). Semiotics: the basics, London [etc.]: Routledge.

Chomsky, N., \& Barsamian, D. (2017). Global Discontents: Conversations on the Rising Threats to Democracy (The American Empire Project), London [etc.]: Penguin Press.

Chupin, J. (2011). Judgement by design: Towards a model for studying and improving the competition process in architecture and urban design. Scandinavian Journal of Management, 27(1), 173-184.

Clarke, A. (2003). Situational Analyses: Grounded Theory Mapping After the Postmodern Turn. Symbolic Interaction, 26(4), 553-576.

Clarke, A. (2005). Situational analysis: grounded theory after the postmodern turn, Thousand Oaks, CA [etc.]: SAGE.

Corner, J. M. (1992). Representation and landscape. Word E Image: A Journal of Verbal/Visual Enquiry, 243-274.

Cortesão, J., Brandão Alves, F., \& Raaphorst, K. (2018). Photographic comparison: a method for qualitative outdoor thermal perception surveys. International Journal of Biometeorology, 1-13.

Cosgrove, D., \& Daniels, S. (1988). The Iconography of Landscape: Essays on the symbolic representation, design and use of past environments, Cambridge: Cambridge University Press.

Council of Europe. (2000). European Landscape Convention. Retrieved July 26, 2017, from https://www.coe.int/en/web/conventions/full-list/-/ conventions/rms/0900001680080621

Crampton, J. W. (2001). Maps as social constructions: power, communication and visualization. Progress in Human Geography, 25(2), 235-252.

Crampton, J. W., \& Krygier, J. (2006). An Introduction to Critical Cartography. ACME, 4(1), 11-33.

Creswell, J., \& Plano Clark, V. (2011). Designing and conducting mixed methods research, Los Angeles: SAGE.

Cridge, N. (2015). Drawing the Unbuildable: Seriality and Reproduction in Architecture, New York: Taylor \& Francis.

Cross, N. (2006). Designerly ways of knowing, London: Springer.

Davison, G., Freestone, R., Hu, R., \& Baker, S. (2018). The impacts of mandatory design competitions on urban design quality in Sydney, Australia. Journal of Urban Design, 23(2), 1-21.

de Boer, A., Breure, L., Spruit, S., \& Voorbij, H. (2011). Virtual historical landscapes. Research in Urbanism Series, 2(1), 185-203.

de Jonge, J. (2009). Landscape architecture between politics and science: an integrative perspective on landscape planning and design in the network society, Blauwdruk / Techne, Wageningen. 
de Waal, R., Stremke, S., van Hoorn, A., Duchhart, I., \& van den Brink, A. (2015). Incorporating Renewable Energy Science in Regional Landscape Design: Results from a Competition in The Netherlands. Sustainability, 7(5), 4806-4828.

Dee, C. (2004). 'The imaginary texture of the real ...' critical visual studies in landscape architecture: contexts, foundations and approaches. Landscape Research, 29(1), 13-30.

Dee, C. (2016). To Draw. Journal of Landscape Architecture, 11(2), 42-53.

Dee, C., \& Fine, R. (2005). Indoors Outdoors at Brightside: A Critical Visual Study Reclaiming Landscape Architecture in the Feminine. Landscape Journal, 24(1), 70-84.

Degen, M., Melhuish, C., \& Rose, G. (2015). Producing place atmospheres digitally: Architecture, digital visualisation practices and the experience economy. Journal of Consumer Culture, 17(1), 3-24.

Deleuze, G., \& Guattari, F. (1987). A Thousand Plateaus, London [etc.]: Bloomsbury.

Deming, M. E., \& Swaffield, S. (2011). Landscape Architecture Research: Inquiry, Strategy, Design, Hoboken, NJ: John Wiley \& Sons.

Denzin, N. K., \& Lincoln, Y. S. (2005). The SAGE handbook of qualitative research, Thousand Oaks, CA [etc.]: SAGE.

Dorling, D., \& Hennig, B. (2015). Visualizing urban and regional worlds: power, politics, and practices. Environment and Planning A, 47(6), 1346-1350.

Dorst, K. (2011). The core of 'design thinking' and its application. Design Studies, 32(6), 521-532.

Downes, M., \& Lange, E. (2015). What you see is not always what you get: A qualitative, comparative analysis of ex ante visualizations with ex post photography of landscape and architectural projects. Landscape and Urban Planning, 142, 136-146.

Duchhart, I. (2007). Designing sustainable landscapes: from experience to theory: a process of reflective learning from case-study projects in Kenya, Wageningen University, Wageningen.

Dühr, S. (2007). The visual language of spatial planning: exploring cartographic representations for spatial planning in Europe, London: Routledge.

Duineveld, M., Van Assche, K., \& Beunen, R. (2017). Re-conceptualising political landscapes after the material turn: a typology of material events. Landscape Research, 42(4), 375-384.

Duncan, J., \& Duncan, N. (1988). (Re)Reading the Landscape. Environment and Planning D: Society and Space, 6(2), 117-126.

Eco, U. (1976). A theory of semiotics, Bloomington: Indiana University Press.

Eco, U. (1990). The Limits of Interpretation, Bloomington [etc]: Indiana University Press.

Eco, U. (1992). Overinterpreting Texts. In S. Collini, eds., Interpretation and overinterpretation, Cambridge, MA: Cambridge University Press, pp. 45-66. 
Feyerabend, P. (1975). Against method, London [etc.]: Verso.

Fleming, B. (2016). Lost in Translation: The Authorship and Argumentation of Resilience Theory. Landscape Journal, 35(1), 23-36.

Foucault, M. (1974). The archaeology of knowledge, London: Tavistok.

Foucault, M. (1980). Power/knowledge: Selected Interviews and Other Writings, 19721977, Brighton: Harvester Wheatsheaf.

Foucault, M. (2000). Governmentality. In J. Faubion, eds., Power, New York: The New Press, pp. 201-222.

Gaberell, S., \& Debarbieux, B. (2014). Mapping regions, framing projects. A comparative analysis on the role of mapping in the region-building process of two European regions. Geoforum, 52, 123-136.

Gailing, L., \& Leibenath, M. (2015). The Social Construction of Landscapes: Two Theoretical Lenses and Their Empirical Applications. Landscape Research, 40(2), 123-138.

Gänshirt, C. (2007). Tools for ideas: introduction to architectural design, Basel [etc]: Birkhäuser.

Gaspard, J. (2015). Discourse analysis with Peirce? Making sense of discursive regularities: The case of online university prospectuses. Semiotica, 207, 551-565.

Gaspard, J. (2016). Discourse genres as determiners of discursive regularities: A case of semiotic predictability? Sign Systems Studies, 44(3), 355-367.

Gaspard, J. (2018). Toward a Peircean Approach to Perlocution. Philosophy E Rhetoric, 51(2), 105-123.

Gill, L., Lange, E., Morgan, E., \& Romano, D. (2013). An analysis of usage of different types of visualisation media within a collaborative planning workshop environment. Environment and Planning B: Planning and Design, 40(4), 742-754.

Glynos, J., \& Howard, D. (2007). Logics of Critical Explanation in Social and Political Theory, London: Routledge.

Gottdiener, M. (1995). Postmodern semiotics: Material culture and the forms of postmodern life, Oxford: Blackwell.

Gough, T. (2016). Are We So Sure It's Not Architecture? Architecture and Culture, 4(1), 9-29.

Gralepois, M., Larrue, C., Wiering, M., ... Szwed, M. (2016). Is flood defense changing in nature? Shifts in the flood defense strategy in six European countries. Ecology and Society, 21(4).

Grinnell, R. (1981). Social Work Research and Evaluation, Itasca, IL: F.E. Peacock.

Groat, L., \& Wang, D. (2013). Architectural research methods, Hoboken, NJ: Wiley.

Groulx, M., \& Lewis, J. L. (2017). Merely 'Design Marketing'?: Professional Perspectives on the Use and Misuse of Environmental Visualizations in Community Engagement. Journal of Planning Education and Research. doi: $10.1177 / 0739456 \times 17739112$

Habermas, J. (1972). Knowledge and human interests, Boston: Beacon Press. 
Hall, S. (1997). Representation: Cultural representations and signifying practices, London: SAGE.

Harley, J. B. (1988). Maps, knowledge, and power. In D. Cosgrove \& S. Daniels, eds., The Iconography of Landscape, Cambridge: Cambridge University Press, pp. 277-312.

Healy, S. (2003). Epistemological pluralism and the 'politics of choice.' Futures, 35(7), 689-701.

Hjelmslev, L. (1953). Prolegomena to a Theory of Language, Baltimore: Waverly Press. Hodge, R., \& Kress, G. (1988). Social Semiotics, Cambridge: Polity Press.

Holdcroft, D. (1991). Saussure: Signs, system and arbitrariness, New York, NY: Cambridge University Press.

Houtkamp, J. (2012). Affective appraisal of virtual environments, Utrecht University, NL.

Howett, C. (1987). Systems, Signs, Sensibilities: Sources for a New Landscape Aesthetic. Landscape Journal, 6(1), 1-12.

Huxley, M. (2008). Space and Government: Governmentality and Geography. Geography Compass, 2(5), 1635-1658.

Iedema, R. (2001). Resemiotization. Semiotica, 137(1/4), 23-39.

Iedema, R. (2003). Multimodality, resemiotization: extending the analysis of discourse as multi-semiotic practice. Visual Communication, 2(1), 29-57.

Interboro. (2014). Living with the Bay: A Comprehensive Regional Resiliency Plan for Nassau County's South Shore, New York.

Jacobs, M. (2004). Metropolitan matterscape, powerscape and mindscape. In G. Tress, B. Tress, B. Harms, P. Smeets, \& A. van der Valk, eds., Planning metropolitan landscapes; concepts, demands, approaches, Wageningen: Wageningen University, pp. 26-38.

Jallouli, J., \& Moreau, G. (2009). An immersive path-based study of wind turbines' landscape: A French case in Plouguin. Renewable Energy, 34(3), 597-607.

Janes, J. E. (2016). The 'Will to Participate': Governmentality, Power, and Community-Based Participatory Research. Intersectionalities: A Global Journal of Social Work Analysis, Research, Polity, and Practice, 5(1), 110-125.

Jappy, T. (2013). Introduction to Peircean visual semiotics, London [etc.]: Bloomsbury. Jay, M. (1993). Downcast Eyes: the Denigration of Vision in Twentieth-Century French Thought, Berkeley, CA: California University Press.

Jenks, C. (1995). The centrality of the eye in Western culture. In C. Jenks, eds., Visual Culture, London: Routledge, pp. 1-12.

Jones, M., \& Stenseke, M. (2011). The Issue of Public Participation in the European Landscape Convention. In M. Jones \& M. Stenseke, eds., The European Landscape Convention, Dordrecht: Springer, pp. 1-23.

Jørgensen, K. (1998). Semiotics in landscape design. Landscape Review, 4(1), 39-47. 
Jørgensen, M., \& Phillips, L. (2002). Discourse analysis: as theory and method, London [etc.]: Sage.

Kallus, R. (2016). Citizenship in action: participatory urban visualization in contested urban space. Journal of Urban Design, 21(5), 616-637.

Kempenaar, A., Westerink, J., van Lierop, M., Brinkhuijsen, M., \& van den Brink, A. (2016). 'Design makes you understand' - Mapping the contributions of designing to regional planning and development. Landscape and Urban Planning, 149, 20-30.

Kendall, G., \& Wickham, G. (1999). Using Foucault's Methods, London [etc.]: SAGE Publications.

Keravel, S. (2010). The art of transmission: mediating meaning in contemporary French landscape design. Journal of Landscape Architecture, 5(1), 60-71.

Kingery-Page, K., \& Hahn, H. (2012). The aesthetics of digital representation: realism, abstraction and kitsch. Journal of Landscape Architecture, 7(2), 68-75.

Kolko, J. (2009). Abductive Thinking and Sensemaking: The Drivers of Design Synthesis. Design Issues, 26(1), 15-28.

Kothuis, B., \& Kok, M. (2017). Integral Design of Multifunctional Flood Defenses, Delft: Delft University Publishers TU Delft Library.

Kress, G. (2009). Multimodality: A social semiotic approach to contemporary communication, London [etc.]: Routledge.

Kress, G., \& van Leeuwen, T. (1996). Reading Images. The Grammar of Visual Design, London [etc.]: Routledge.

Kullmann, K. (2014). Hyper-realism and loose-reality: the limitations of digital realism and alternative principles in landscape design visualization. Journal of Landscape Architecture, 9(3), 20-31.

Kumar, R. (2014). Research Methodology, London: SAGE.

Lacan, J. (1977). Écrits: A Selection, London [etc.]: Routledge.

Laclau, E., \& Mouffe, C. (2014). Hegemony and socialist strategy: towards a radical democratic politics, London: Verso.

Lang, J. (1987). Creating architectural theory: the role of the behavioral sciences in environmental design, New York: Van Nostrand Reinhold.

Lange, E. (2011). 99 volumes later: We can visualise. Now what? Landscape and Urban Planning, 100(4), 403-406.

Laurie, M. (1975). An introduction to landscape architecture, New York: American Elsevier.

Lawson, B. (2005). How Designers Think: The Design Process Demystified, Amsterdam: Elsevier Architectural Press.

Lawson, B., \& Dorst, K. (2013). Design expertise, Oxford: Taylor \& Francis.

Lee, M., \& Pae, J. (2017). Photo-fake conditions of digital landscape representation. Visual Communication, 17(1), 3-23. 
Lenzholzer, S., \& Brown, R. D. (2013). Climate-responsive landscape architecture design education. Journal of Cleaner Production, 61, 89-99.

Lenzholzer, S., Duchhart, I., \& Koh, J. (2013). 'Research through designing' in landscape architecture. Landscape and Urban Planning, 113, 120-127.

Lewins, A., \& Silver, C. (2007). Using Software in Qualitative Research: A Step-byStep Guide, Los Angeles, CA [etc.]: SAGE Publications Ltd.

Lewis, J. L. (2012). More art than science: The sources and effects of stylistic variation in visualization for planning and design. Environment and Planning B: Planning and Design, 39(3), 551-565.

Lewis, J. L., Casello, J. M., \& Groulx, M. (2012). Effective Environmental Visualization for Urban Planning and Design: Interdisciplinary Reflections on a Rapidly Evolving Technology. Journal of Urban Technology, 19(3), 85-106.

Lindström, K., Kull, K., \& Palang, H. (2014). Landscape semiotics: Contribution to culture theory. In K. Kull \& V. Lang, eds., Estonian Approaches to Culture Theory, Tartu: University of Tartu Press, pp. 110-135.

Lindström, K., Palang, H., \& Kull, K. (2013). Semiotics of landscape. In P. Howard, I. Thompson, \& E. Waterton, eds., The Routledge companion to landscape studies, London: Routledge, pp. 97-107.

Lovett, A., Appleton, K., Warren-Kretzschmar, B., \& Von Haaren, C. (2015). Using $3 \mathrm{D}$ visualization methods in landscape planning: An evaluation of options and practical issues. Landscape and Urban Planning, 142, 85-94.

Macdonald, A. (2012). Windfarm Visualisation: Perspective or Perception?, Dunbeath: Whittles.

MacEachren, A. M. (1995). How maps work: representation, visualization, and design, New York: Guilford.

MacHarg, I. L. (1969). Design with nature, New York: Natural History Press.

Marttila, T. (2016). Post-foundational discourse analysis: from political difference to empirical research, Houndmills [etc]: Palgrave Macmillan.

Matos Castaño, J., Hartmann, T., Dewulf, G. P. M. R., \& van Huffelen-de Kort, I. A. T. (2015). 'What is going on and what should we do?' Divergent frames in multifunctional projects. Engineering Project Organization Journal, 5(1), 36-48.

Menatti, L. (2011). From non-place to rhizome: A geophilosophical analysis of contemporary globalized space. Environment, Space, Place, 3, 22-50.

Mertens, E., Robinson, M., \& Kirkland, A. (2010). Visualizing landscape architecture: functions, concepts, strategies, Basel: Birkhäuser Verlag.

Metz, C. (1973). Methodological Propositions for the Analysis of Film. Screen, 14(1-2), 89-101.

Miles, M. B., Huberman, A. M., \& Saldaña, J. (2014). Qualitative data analysis: a methods sourcebook, Los Angeles, CA [etc.]: SAGE.

MIT-CAU, ZUS, \& Urbanisten. (2014). The New Meadowlands, Cambridge, MA.

Mitchell, W. T. J. (2002). Landscape and Power, Chicago, IL: SAGE. 
Monmonier, M. (1995). Drawing the line: Tales of maps and cartocontroversy, New York, NY: Henry Holt.

Monmonier, M. (1996). How to lie with maps, Chicago: The University of Chicago Press.

Morris, C. (1938). Foundations of the theory of signs, Chicago, IL: University of Chicago Press.

Morrison, T. (2015). Unbuilt Utopian Cities 1460 to 1900: Reconstructing their Architecture and Political Philosophy, London: Routledge.

Müller, M. G. (2011). Iconography and iconology as a visual method and approach. In The SAGE handbook of visual research methods, London: SAGE, pp. 283-297.

Nassauer, J. I. (2015). Commentary: Visualization verisimilitude and civic participation. Landscape and Urban Planning, 142, 170-172.

Ndubisi, F. (2014). The ecological design and planning reader, Washington, DC: Island Press.

Nietzsche, F. (1967). The Will To Power, New York: Vintage Books.

Nijhuis, S. (2015). GIS-based landscape design research: stourhead landscape garden as a case study, Delft University of Technology, NL.

OMA. (2014). Resist, Delay, Store, Discharge: a comprehensive urban water strategy, New York, NY.

OntwikkelingsBedrijf Rotterdam. (2003). Masterplan 'Het Dakpark', Rotterdam.

Ovink, H. (2017). Rebuild by Design: New Approaches to Climate Change, Rotterdam: NAi Uitgevers.

Ovink, H., \& Boeijenga, J. (2016). Rebuild by Design: New Approaches to Climate Change, Rotterdam: nai010 Publishers.

Paar, P. (2006). Landscape visualizations: Applications and requirements of 3D visualization software for environmental planning. Computers, Environment and Urban Systems, 30(6), 815-839.

Pada, R. T. S. (2009). Iterability and Différance: Re-tracing the Context of the Text. Kritike, 3(2), 68-89.

Pallasmaa, J. (2009). The thinking hand: existential and embodied wisdom in architecture, Chichester: Wiley.

Panofsky, E. (1955). Meaning in the visual arts: papers in and on art history, Garden City, NY: Doubleday Anchor Books.

Parret, H. (1984). Peirce and Hjelmslev: The two semiotics. Language Sciences, 6(2), 217-227.

Peirce, C. S. (1958). Collected Papers of Charles Saunders Peirce. (C. Hartshorne \& P. Weiss, Eds.), Cambridge, MA: Harvard University Press. 
Peirce, C. S. (1992). How to Make Our Ideas Clear. In N. Houser \& C. Kloesel, eds., The Essential Peirce, Volume 1: Selected Philosophical Writings (1867-1893), Bloomington [etc]: Indiana University Press, pp. 124-141.

Perkins, N., \& Barnhart, S. (2005). Visualization and Participatory Decision-Making. In I. D. Bishop, eds., Visualization in Landscape and Environmental Planning: Technology and Applications, London: Taylor \& Francis, pp. 241-250.

Peters, V., \& Wester, F. (2007). How Qualitative Data Analysis Software may Support the Qualitative Analysis Process. Quality \& Quantity, 41(5), 635-659.

Petrow, C. A. (2011). Hidden meanings, obvious messages: landscape architecture as a reflection of a city's self-conception and image strategy. Journal of Landscape Architecture, 6(1), 6-19.

Pink, S. (2012). Advances in visual methodology, London [etc.]: SAGE.

Pojani, D., \& Stead, D. (2016). Post-rational planning and the shifting role of planning imagery. Journal of Urban Design, 21(3), 353-385.

Popper, K. R. (1972). Objective Knowledge: an evolutionary approach, London [etc.]: Oxford University Press.

Potteiger, M., \& Purinton, J. (1998). Landscape Narratives: Design Practices for Telling Stories, New York [etc.]: Wiley.

Proto, F. (2003). Mass identity architecture: Architectural writings of Jean Baudrillard, London: Wiley.

Raaphorst, K. (2017). 'Deconstructing' the Rotterdam roof park. In B. Kothuis \& M. Kok, eds., Integral design of multifunctional flood defenses, Delft: Delft University Publishers, pp. 174-177.

Raaphorst, K. (2018). Knowing your audience: the contingency of landscape design interpretations. Journal of Urban Design, 23(5), 1-20.

Raaphorst, K., Duchhart, I., van der Knaap, W., Roeleveld, G., \& van den Brink, A. (2017). The semiotics of landscape design communication: towards a critical visual research approach in landscape architecture. Landscape Research, 42(1), 120-133.

Raaphorst, K., Roeleveld, G., Duchhart, I., van der Knaap, W., \& van den Brink, A. (2018). Reading landscape design representations as an interplay of validity, readability, and interactivity: a framework for visual content analysis. Visual Communication, Online first.

Rattenbury, K. (2002). This is Not Architecture: Media Constructions, London: Routledge.

Rebuild by Design. (2013). Promoting Resilience Post-Sandy Through Innovative Planning and Design. Retrieved August 8, 2017, from https://portal.hud.gov/ hudportal/documents/huddoc?id=REBUILDBYDESIGNBrief.pdf

Rebuild by Design. (2015). Rebuild by Design, New York: Fergus.

Rijksoverheid. (2017). Deltaprogramma: bescherming tegen overstromingen en zoetwatertekort. Retrieved August 8, 2017, from https://www.rijksoverheid. 
$\mathrm{nl}$ /onderwerpen/deltaprogramma/deltaprogramma-bescherming-tegenoverstromingen-en-zoetwatertekort

Rose, G. (2012). Visual Methodologies: An Introduction to Researching with Visual Materials, London [etc.]: SAGE.

Rose, N. (1999). Powers of Freedom: Reframing Political Thought, Cambridge: Cambridge University Press.

Ruotolo, F., Maffei, L., Di Gabriele, M., ... Senese, V. P. (2013). Immersive virtual reality and environmental noise assessment: An innovative audio-visual approach. Environmental Impact Assessment Review, 41, 10-20.

Salter, J. D., Campbell, C., Journeay, M., \& Sheppard, S. R. J. (2009). The digital workshop: Exploring the use of interactive and immersive visualisation tools in participatory planning. Journal of Environmental Management, 90(6), 2090-2101.

Scheffler, I. (2012). Four Pragmatists: A Critical Introduction to Peirce, James, Mead, and Dewey, Routledge, London: Taylor \& Francis.

Schroth, O., Hayek, U. W., Lange, E., Sheppard, S. R. J., \& Schmid, W. A. (2011). Multiple-Case Study of Landscape Visualizations as a Tool in Transdisciplinary Planning Workshops. Landscape Journal, 30(1), 53-71.

Schwartz-Shea, P., \& Yanow, D. (2012). Interpretive Research Design: Concepts and Processes, London: Routledge.

Scollon, R. (2008). Discourse itineraries. Nine processes of resemiotization. In V. K. Bhatia, J. Flowerdew, \& R. H. Jones, eds., Advances in discourse studies, London [etc.]: Routledge, pp. 233-244.

Sheppard, S. (2001). Guidance for crystal ball gazers: Developing a code of ethics for landscape visualization. Landscape and Urban Planning, 54(1-4), 183-199.

Sheppard, S., Shaw, A., Flanders, D., ... Cohen, S. (2011). Future visioning of local climate change: A framework for community engagement and planning with scenarios and visualisation. Futures, 43(4), 400-412.

Sijmons, D. (1990). Regional planning as a strategy. Landscape and Urban Planning, 18(3), 265-273.

Silverman, D. (2001). Interpreting qualitative data: methods for analyzing talk, text and interaction, London: SAGE.

Simonds, J. O. (1998). Landscape Architecture: A Manual of Site Planning and Design, New York [etc.]: McGraw-Hill.

Sletto, B. I. (2009). 'We Drew What We Imagined' Participatory Mapping, Performance, and the Arts of Landscape Making. Current Anthropology, 50(4), 443-476.

Southworth, M., Cranz, G., Lindsay, G., \& Morhayim, L. (2012). People in the Design of Urban Places. Journal of Urban Design, 17(4), 461-465.

Spencer, S. (2011). Visual research methods in the social sciences: Awakening visions, London: Routledge. 
Spirn, A. W. (1998). The Language of Landscape, New Haven [etc.]: Yale University Press.

Steiner, F. R. (2006). The Essential Ian McHarg: Writings on Design and Nature, Washington: Island Press.

Steinitz, C. (2012). A framework for Geodesign: changing geography by design, Redlands, CA: ESRI.

Sternberg, E. (1996). A case of iconographic competition: The building industry and the postmodern landscape. Journal of Urban Design, 1(2), 145-163.

Swords, J., \& Liu, X. (2015). Visualizing urban and regional worlds: power, politics, and practices. Environment and Planning A, 47(6), 1235-1240.

Taverniers, M. (2008). Hjelmslev's semiotic model of language: An exegesis. Semiotica, 2008(171), 367-394.

Taylor, J. C. (1981). Learning to Look: A Handbook for the Visual Arts, London: University of Chicago Press.

Thering, S., \& Chanse, V. (2011). The Scholarship of Transdisciplinary Action Research: Toward a New Paradigm for the Planning and Design Professions. Landscape Journal, 30(1), 6-18.

Tobias, S., Buser, T., \& Buchecker, M. (2015). Does real-time visualization support local stakeholders in developing landscape visions? Environment and Planning B: Planning and Design, 43(1), 184-197.

Torfing, J. (2005). Discourse theory: Achievements, arguments, and challenges. In D. Howarth \& J. Torfing, eds., Discourse theory in European politics, Springer, pp. $1-32$.

Törrönen, J. (2002). Semiotic theory on qualitative interviewing using stimulus texts. Qualitative Research, 2(3), 343-362.

Treib, M. (2008). Representing Landscape Architecture, London [etc.]: Taylor \& Francis.

Tufte, E. R. (1990). Envisioning information, Cheshire, CO: Graphics Press.

van Assche, K., Duineveld, M., de Jong, H., \& van Zoest, A. (2012). What place is this time? Semiotics and the analysis of historical references in landscape architecture. Journal of Urban Design, 17(2), 233-254.

van Buuren, A., Ellen, G. J., \& Warner, J. F. (2016). Path-dependency and policy learning in the Dutch delta. Ecology and Society, 21(4).

van de Ven, F., Gersonius, B., de Graaf, R., Luijendijk, E., \& Zevenbergen, C. (2011). Creating water robust urban environments in the Netherlands: Linking spatial planning, design and asset management using a three-step approach. Journal of Flood Risk Management, 4(4), 273-280.

van den Brink, A., \& Bruns, D. (2014). Strategies for Enhancing Landscape Architecture Research. Landscape Research, 39(1), 7-20. 
van den Brink, A., van Lammeren, R., van de Velde, R., \& Däne, S. (2007). Imaging the future: geo-visualisation for participatory spatial planning in Europe, Wageningen: Wageningen Academic Publishers.

van der Knaap, W. (1997). The tourist's drives, Wageningen University, NL.

van der Stoep, H., Aarts, N., \& van den Brink, A. (2017). Shifting frames: mobilizing policy attention for landscape values in a Dutch urban-rural fringe. Journal of Environmental Policy \& Planning, 19(6), 697-711.

van Dijk, T. (2011). Imagining future places: How designs co-constitute what is, and thus influence what will be. Planning Theory, 10, 124-143.

van Dooren, N. (2017). Drawing Time: The representation of growth, change and dynamics in Dutch landscape architectural practice after 1985, University of Amsterdam, NL.

van Etteger, R., Thompson, I. H., \& Vicenzotti, V. (2016). Aesthetic creation theory and landscape architecture. Journal of Landscape Architecture, 11(1), 80-91.

van Houtum, H. (2013). Van Atlas naar Hermes: Pleidooi voor een bevrijding van de cartografie [From Atlas towards Hermes: A plea for the liberation of cartography]. Geografie. Vaktijdschrift Voor Geografen, 22, 96-99.

van Lammeren, R., Houtkamp, J., Colijn, S., Hilferink, M., \& Bouwman, A. (2010). Affective appraisal of 3D land use visualization. Computers, Environment and Urban Systems, 34(6), 465-475.

van Leeuwen, T. (2001). Semiotics and iconography. In Handbook of Visual Analysis, London: SAGE, pp. 92-118.

van Leeuwen, T. (2005). Introducing Social Semiotics. New York, London: Routledge. van Leeuwen, T., \& Jewitt, C. (2001). Handbook of visual analysis, London: SAGE.

van Veelen, P., Voorendt, M., \& van der Zwet, C. (2015). Design challenges of multifunctional flood defences. A comparative approach to assess spatial and structural integration. Research in Urbanism Series; Vol 3 (2015): Flowscapes: Designing Infrastructure as Landscape, 3, 275-292.

van Wezemael, J. (2011). Research on architectural competitions: Towards a theory of jury-based decision-making. Scandinavian Journal of Management, 27(1), 157-159.

Vannini, P. (2007). Social Semiotics and Fieldwork: Method and Analytics. Qualitative Inquiry, 13(1), 113-140.

Verduijn, S. H., Meijerink, S. V, \& Leroy, P. (2012). How the second delta committee set the agenda for climate adaptation policy: A dutch case study on framing strategies for policy change. Water Alternatives, 5(2), 469-484.

Vonk, G., \& Ligtenberg, A. (2010). Socio-technical PSS development to improve functionality and usability - Sketch planning using a Maptable. Landscape and Urban Planning, 94(3), 166-174.

Voorendt, M. (2017). Design principles of multifunctional flood defences, Delft University of Technology, NL.

Vroom, M. (2006). Lexicon of garden and landscape architecture, Basel: Birkhäuser. 
Vroom, M. J. (1990). Landscape Planning in the Netherlands: the Role of Competitions. Built Environment, 16(2), 141-161.

Walliss, J., Hong, Z., Rahmann, H., \& Sieweke, J. (2014). Pedagogical foundations: deploying digital techniques in design / research practice. Journal of Landscape Architecture, 9(3), 72-83.

Walliss, J., \& Rahmann, H. (2016). Landscape Architecture and Digital Technologies: re-conceptualizing design and making, London [etc.]: Routledge.

Watson, D., \& Adams, M. (2010). Design for flooding: architecture, landscape, and urban design for resilience to flooding and climate change, Hoboken, New Jersey: John Wiley \& Sons.

Williams, K. J. H., Ford, R. M., Bishop, I. D., Loiterton, D., \& Hickey, J. (2007). Realism and selectivity in data-driven visualisations: A process for developing viewer-oriented landscape surrogates. Landscape and Urban Planning, 81(3), 213-224.

Wohl, R. R., \& Strauss, A. L. (1958). Symbolic representation and the urban milieu. American Journal of Sociology, 63, 523-532.

Wood, D. (2003). Cartography is dead (thank God!). Cartographic Perspectives, $45(45), 4-7$.

Wylie, J. (2005). A single day's walking: narrating self and landscape on the South West Coast Path. Transactions of the Institute of British Geographers, 30(2), 234-247.

Yanow, D., \& Schwartz-Shea, P. (2014). Interpretation and method: empirical research methods and the interpretive turn, Armonk, NY [etc.]: M.E. Sharp.

Yin, R. K. (2009). Case Study Research. Design and Methods, Los Angeles, CA [etc.]: SAGE.

Žižek, S. (2015). Trouble in Paradise: From the End of History to the End of Capitalism, Brooklyn: Melville House. 


\section{Appendices}

\section{Appendix A. List of Interviews}

1. Ecologist local university in New Jersey

2. Landscape architect local university in New Jersey

3. Project developer in New Jersey

4. Environmental protectionist in New Jersey

5. Landscape architect from BIG-U team

6. Lead Urban Designer OMA team

7. Project leader municipality OMA team

8. Lead organizer Citizens Advisory Group OMA team

9. Urban designer from MIT-CAU+ZUS+Urbanisten team

10. Ecological designer from MIT-CAU+ZUS+Urbanisten team

11. Landscape architect from MIT-CAU+ZUS+Urbanisten team

12. Water expert from the MIT-CAU+ZUS+Urbanisten team

13. Water expert from the Interboro team

14. Landscape architect (1) from the Interboro team

15. Landscape architect (2) from the Interboro team

16. Rebuild by Design assistant organizer

17. Academic expert on flood adaptation and real estate markets

18. Urban designer Deltares

19. Landscape architect (1) Deltares

20. Landscape architect (2) Deltares 


\section{Appendix B. List of Figures}

Figure 1.1. Old situation of the Rotterdam 'Roof Park': project area marked in red (OntwikkelingsBedrijf Rotterdam, 2003).

Figure 1.2. Early conceptual drawing of the Rotterdam 'Roof Park' (Adapted from OntwikkelingsBedrijf Rotterdam, 2003).

Figure 1.3. Early scale model of the Rotterdam 'Roof Park' (Ontwikkelingsbedrijf Rotterdam, 2003).

Figure 1.4. Bird's eye drawing of the 'Parklane' concept (Ontwikkelingsbedrijf Rotterdam, 2003).

Figure 1.5. 3D rendering of the 'Park Shops Boulevard' (Buro Sant en Co, 2007

Figure 1.6. Peirce's concept of the sign as a function.

Figure 1.7. Concept of a visual communication process (own figure, adapted from Peirce, Figure 1.6).

Figure 2.1. Dyadic Model of Semiosis by de Saussure (own figure).

Figure 2.2. Singular (left) and multiple (right) signification (own figure).

Figure 2.3. Triadic model of semiosis of Charles Sanders Peirce (own figure).

Figure 2.4. Possible assemblage of meaning through a triadic signifying chain of connotation (own figure).

Figure 2.5. Denotative Meaning in a Photomontage (BMD, 2011) (own analysis).

Figure 2.6. Denotative Meaning and Possible Connotations in Photomontage (BMD, 2011) (own analysis).

Figure 3.1. Example of schematic visual representation showing landscape systemics (Winder, 2014).

Figure 3.2. Example of atmospherical visual representation showing the potential experience of a flood event (Valcarlos, 2014).

Figure 3.3. Example of a visual representation that shows the historical narrative of a landscape (van Zuiden, 2016). 
Figure 3.4. Hybrid Semiotics (based on Eco): dyadic theory of Hjelmslev embedded in the triadic logic of Peirce (own figure).

Figure 3.5. Syntactic relationships of validity, readability, and interactivity (own figure).

Figure 3.6. Analytic framework: combining iconography with Peirce, Eco \& Hjelmslev (own figure).

Figure 3.7. Validity: an image with an analytical formulation (MIT-CAU et al. 2014).

Figure 3.8. Readability: an analytical image with an atmospherical style (Interboro, 2014).

Figure 3.9. Interactivity: a board presentation with singular visual techniques (MIT-CAU et al. 2014).

Figure 4.1. Example of a discursive network and its components (own figure, adapted from Marttila (2016) and Clarke (2005).

Figure 4.2. Formation of the 'clean water act' nodal point (own figure).

Figure 4.3. Formation of 'Environmental Preservation' discourse (own figure).

Figure 4.4. Example of a map that depicts the 'Preliminary Flood Protection Alignments" in a part of the Meadowlands. The proposed location of a berm through the conservation land is depicted in yellow. (New Jersey Department of Environmental Protection).

Figure 4.5. Discursive formation of 'environmental preservation' and 'grassroots movement' (own figure).

Figure 4.6. Example of Photoshop rendering of urban developments along the Hackensack river in the Meadowlands (MIT-CAU et al., 2014).

Figure 5.1. Conceptual framework for landscape design communication (Raaphorst et al. (2018).

Figure 5.2. Conceptual framework for visual discourse in landscape design representations (Adapted from Raaphorst et al. (2018)).

Figure 5.3. Three conceptualized aspects of visual discourse for participatory planning and design. (Image adapted from MIT-CAU et al. (2014)).

Figure 5.4. Flood risk pamphlet produced by the Hoboken project team (OMA 2014).

Figure 5.5. Paper sketch without anchorage (top) vs. digitized drawing with anchorage of later iteration (bottom) (Interboro, 2014). 
Figure 5.6. Visual rhetoric: examples of the 'resist, delay, store, discharge' rhetoric in the Hoboken design proposal (OMA, 2014).

Figure 5.7. Poster boards of the Meadowlands project team. (MIT-CAU et al., 2014).

Figure 5.8. Poster boards of the Long Island project proposal (Interboro, 2014).

Figure 6.1. Analytical framework of a critical visual research approach to studying the meaning-making processes of visual landscape design representations (own figure).

Figure 6.2. Representational semiotic qualities of design representations (own figure).

Figure 6.3. Symbolic qualities of design representations (own figure).

Figure 6.4. Existential qualities of design representations (own figure). 


\section{Appendix C. List of Tables}

Table 1.1. Selection of embedded case studies.

Table 1.2. Research methods and data collection methods for all four research foci.

Table 1.3. Overview of thesis structure in chapters and peer-reviewed journal articles.

Table 2.1. Comparison of Semiotic Concepts. Source: own figure.

Table 3.1. Operationalization of Hjelmslev's categories for landscape design representations.

Table 3.2. Categories and variables identified from Kress \& van Leeuwen (2006), Taylor (1981), Acton (1997), Lawson (2006), Simonds (1998), and Lang (1987).

Table 3.3. Coding scheme and code occurrences for the categories, variables, and values used in the visual content analysis of landscape design representations for each $\mathrm{RbD}$ project.

Table 3.4. Co-occurrence table of formulation and style codes of the Interboro project.

Table 3.5. Co-occurrence table of technique and explanational codes of the Interboro project.

Table 3.6. Codes attributed to the image of Figure 7. Relevant code signified by dashed borders.

Table 3.7. Codes attributed to the image of Figure 8. Relevant codes signified by dashed borders.

Table 3.8. Codes attributed to the image in Figure 9. Relevant codes signified by dashed borders.

Table 5.1 Visual discourse analytical framework.

Table 5.2 Overview of RbD proposals used for visual discourse analysis.

Table 6.1. Nine combinations of communicative qualities and sign-functions.

Table 6.2. Definitions and empirical examples of representational validity, readability and interactivity.

Table 6.3. Definitions and empirical examples of symbolic validity, readability and interactivity.

Table 6.4. Definitions and empirical examples of existential validity, readability and interactivity. 



\section{Summary}

This thesis investigates the communicative qualities of visual landscape design representations in a participatory context. The need for this thesis research originated from the observation that landscape planning and design projects, especially those related to climate change adaptation, require multifunctional solutions. This multifunctionality, combined with an increasing level of public participation, leads to the inclusion of a variety of experts, stakeholders and local communities. Visual representations are the primary means of communication between designers, planners, and participants during such participatory processes yet little critical research has been undertaken on how different forms of visual design communication work or what their socio-political implications are for the progress and outcomes of participatory planning and design processes.

The research objective of this thesis was to contribute to a more conscious use of visual design representations in participatory landscape planning and design processes by studying the visual communication that occurs between designers, experts and stakeholders as meaning-making processes. Identifying the influence of these meaning-making processes on the progress and outcome of participatory planning and design projects enables more effective and transparent communication between project stakeholders.

The research objective identified in this thesis translates into the following research question:

What meaning-making processes are visual landscape design representations part

of, and how do these processes shape the progress and outcomes of participatory landscape planning and design projects?

In this thesis, I focused on the use of visual design representations as a means of communication within participatory planning and design processes 
of multifunctional flood defence landscapes. The main subjects of inquiry were therefore (1) visual design representations in their many forms and appearances; (2) the meanings that are attributed to or derived from these representations by designers, planners, and project stakeholders; (3) the participatory planning and design processes of multifunctional flood defence landscape projects where these representations are used. To study these subjects, theoretical perspectives on meaning-making and visual research methods were explored and applied, and a critical visual research approach to the study of the meanings of visual landscape design representations in a participatory context was developed.

The relation between visual design representations, their production and their interpretation is considered as a process of meaning-making. This process of meaning-making is called semiosis and the study of this process is called semiotics. As meaning-conveying objects, visual design representations and their influence on participatory planning and design processes are conceptualized through a theoretical perspective that is based on the Peircean theory of semiotics. For semioticians, the meaning of the world is mediated by different kinds of signs: sounds, smells, colours, emotions, sentiments, etc. Everything has the potential to be a sign as long as it means something to someone. In Peircean semiotics three components of the sign are distinguished: representamen, object and interpretant. The representamen consists of the perceivable form of a sign, i.e. its physical manifestation as a sound, smell, form, or image. The object consists of the thing that the sign refers to. For example, a flood map (representamen) represents the extent in which an area was or could be flooded (object). The interpretant consists of the interpretation of that object by someone via its representamen. In turn, that interpretation leads to an action or influence a result which is also described as the critical function of a sign. This triadic perspective constitutes the main conceptual framework of the research carried out in this thesis. Visual design representations, as signs that contain a great number of signs within themselves, are studied from 
four perspectives: their critical functions, their representamen, their interpretation, and their object.

Firstly, the outline of a critical visual research approach for studying the influence of landscape design representations on participatory planning and design processes has been presented. This outline based on a literature study shows that concepts of visual and critical social theory (e.g. visual semiotics, simulacra and simulation, and power/knowledge) are useful for critically reflecting on landscape architectural representations. It has also been proposed to study these representations at different stages of meaning-making with different visual methodologies, e.g. iconographical content analysis, social semiotic analysis and visual discourse analysis. These research approaches have the potential to explain issues such as dominant power structures, miscommunication between participants, and visual path-dependencies during landscape design processes.

The proposed critical visual research approach was then applied to each of the so-called 'stages of visual communication', i.e. image, audiencing or interpretation and production. These stages relate to the aforementioned sign components, i.e. representamen, interpretant and object, respectively. The image stage was studied in terms of its semiotic complexity, the audiencing or interpretation stage was studied through the analysis of interpretive habit, and the production stage was analysed by studying the visual discourse embedded in the image during its production process.

The study of visual design representations and the meaning-making processes that they are part of was conducted using an explorative retrospective case study approach. The empirical case study that is representative of the subjects of inquiry, i.e. visual design representations and the participatory context in which they were produced and interpreted was identified in the form of the design processes and representations that were part of the Rebuild by Design (RbD) competition. $\mathrm{RbD}$ was a transdisciplinary design competition that took place in the greater metropolitan area of New York after 2012's hurricane Sandy. The process of visual 
communication - or visual semiosis - was studied by analysing the images that were produced during these design processes, and by interviewing the audiences and producers of those images.

The semiotic complexity of 759 landscape design representations was studied using an analytical framework based on semiotic and iconographic theory. This framework enables a visual content analysis and iconographic interpretation of two projects from Rebuild by Design, i.e. the New Meadowlands and the Living With the Bay projects. This framework also presents a semiotic vocabulary based on four categories, i.e. medium, mode, formulation, and knowledge, with which to 'read', discuss and potentially create design representations. These categories enable the assessment of the semiotic complexity of design representations in terms of validity, readability and interactivity. The validity of design ideas relates to the functionality, visual aesthetics, and feasibility of the design, i.e. whether the solutions are suitable for arriving at a multifunctional flood defence landscape; whether these solutions are deemed necessary and implementable; whether they are supported by the process participants. The readability of a design image relates to the extent to which participants can read and understand the content of a design. The interactivity of a design image relates to the medium through which it is presented and the extent to which that medium allows the participants to interact with the content and visualization of the design. What is considered to be valid, readable, and interactive depends on both the interpretive habits of the audience and the producer's discursive power to influence these habits.

The interpretive habits of landscape design representations were studied using an analytical framework for a social semiotic post-foundational discourse analysis (SSPDA). This framework was applied to a partial reconstruction of the sociopolitical context of three Rebuild by Design projects, i.e. the New Meadowlands, the Resist, Delay, Store, Discharge, and the Big-U projects. By analysing the interviews with designers and local stakeholders counter-productive interpretations of landscape designs were uncovered which could easily have been anticipated by the 
designers at the moment of creation. By recognizing such conflicting interpretive habits during specific phases of the design process, planners and designers are able to better anticipate the productive and counter-productive interpretations of their designs. Consequently, planners and designers can attune their visual representations to their audiences' interpretive habits or try to influence these habits through the use of the discursive power of their representations.

This visual discourse of landscape design representations was studied by conceptualizing landscape design representations as discursive materializations of power and knowledge. The social relations within which the production of representations is embedded and the way that these relations manifest themselves in the communicative qualities of design representations were studied using a visual discourse analysis. The visual discourse analysis of Rebuild by Design uncovered interdependencies between three components of visual discourse: the arrangement of the participatory process, the interactivity of the media being used and the visual rhetoric embedded within the composition and style of the image. A conscious use of these discursive components by planners and designers prevents miscommunication, manages participant expectations and increases the validity of participatory design process outcomes.

The answer to the first part of the main research question of this thesis consists of a tested framework for a critical visual research approach. Using this approach, the meaning-making processes of visual design representations are conceptualized in terms of being a relationship between the semiotic complexity, interpretive habits and visual discourse of design representations. The semiotic complexity of images gives rise to varying degrees of validity, readability and interactivity. Mapping the interpretive habits of specific project stakeholders enables a contingent anticipation of how the validity, readability and interactivity of a design representation will be evaluated and acted upon. These interpretive habits are constantly influenced by signs and sign systems that have discursive 
power. The production of design images is, therefore, conceptualized in terms of this discursive power, i.e. its visual discourse.

The answer to the second part of the main research question of this thesis is derived from the application of the critical visual research approach to visual representations produced during the Rebuild by Design competition. As a result, the influence of the meaning-making processes of design representations is categorized into a typology of nine semiotic qualities. This typology uses the distinction of the three communicational elements of semiotic complexity, i.e. validity, readability, and interactivity, combined with the semiotic perspective of the critical visual research approach of this thesis. This perspective stresses the importance of distinguishing between representational, existential and symbolic sign-functions of those communicational elements.

Representational functions refer to the qualities and characteristics of a design, i.e. whether a design representation is actually considered to be valid, readable and interactive. Representational validity relates to the extent that the design's content represents the knowledge and preferences of the project participants. Representational readability relates to the extent that knowledge and ideas contributed by participants can be read and understood by at least those participants. Representational interactivity relates to the extent that the openness of the medium represents the way that participants are supposed to share their knowledge and perceive the design's content.

The symbolic or connotative and rhetorical qualities of an image influence whether those qualities are actually present, or whether they are perceived to be more or less present than they actually are. Symbolic validity relates to the extent that participants perceive a design's validity not in terms of its content, e.g. function, visual impact, or feasibility but in terms of its credibility and authority. Symbolic readability relates to the extent that the style or visual rhetoric of an image connects to or is in conflict with the interpretive habits of its audience. Symbolic interactivity 
relates to the extent that the perceived openness of a medium is coherent with the degree of participation that participants actually have.

The inclusion of the existential function forces one to always consider the path of origin and the consequences of choosing for certain representational and symbolic elements for the progress and implementation of a design. Existential validity relates to the extent that the validity of the design's content contributes to or detracts from the progress of the planning and design process. This is the case whenever a particular stakeholder is needed for an 'institutional buy-in' to advance the process. Similarly, powerful stakeholders can also seriously derail a project if they consider the content invalid from their perspective. Existential readability relates to the extent that the ability of participants to read and understand the visualization contributes to or detracts from the progress of the planning and design process. An example of positive existential readability would be the development of a shared visual language amongst stakeholders during a participatory process. Existential interactivity relates to the extent that the ability of participants to engage with the medium of representation contributes to or detracts from the progress of the planning and design process. This category would, for example, justify the training of participants in the use of particular media, such as a map table or virtual reality glasses, so they would not feel excluded.

The critical visual research approach, analytical framework, and typology of semiotic qualities presented in this thesis offer a way of dealing with the situational complexity of participatory multifunctional landscape projects. For example, the semiotic vocabulary that is used to deconstruct the semiotic complexity of images exceeds a mere categorization of content or visual techniques. Rather, it promotes the idea that each colour, perspective, textual element, drawing technique or medium is a sign in itself. As signs, each of these variables influences the interpretation of the image as a whole and therefore the interpretation of the design content which is being represented. Moreover, attention is paid explicitly to the process of interpretation and the socio-political context that influences the interpretive 
habits' of project stakeholders. In establishing such a strong connection between the semiotic complexity of images and the socio-political context of the design process, interpretations are no longer considered a black box of unpredictable responses. Instead, they consist of a contingent range of possibilities which can be anticipated and acted upon by the producers of design representations. Finally, the influence that design imagery has on the interpretive habits of its audience is explained in this thesis from a discursive viewpoint. Visual discourse, by its conceptual nature, struggles for assimilation and domination within the discursive networks that make up the socio-political context of participatory design processes. In recognizing such discursive powers, the influence of planners and designers on the interpretations of their representations can be identified more clearly and used more consciously.

This thesis suggests a way of looking at visual representations and organizing the planning and design process in ways that enable planners and designers, as process facilitators, to determine the most appropriate communicative strategy at a specific moment, for specific participants. For a visual representation to be effective and communicate successfully, validity, readability and interactivity all need to be considered. In practice, the details will depend on the nature of the project, on the stakeholders involved and on how their participation is organized. Nevertheless, a keen eye for the - sometimes - subtle distinction between representational and symbolic qualities will help planners and designers to separate facts from fiction, strong visual narratives from window dressing and genuine participation tools from technological gimmicks. Existential qualities help trace the origin of certain ideas, determine the visual language that participants are used to and assess the level of participation used in previous design phases. At the same time, planners and designers are forced to look ahead: what pathways are chosen, how fixed are they and what kind of productive and counterproductive interpretations are to be expected? 




\section{Samenvatting}

Dit proefschrift behandelt de communicatieve eigenschappen van visuele representaties van landschapsontwerpen binnen de context van participatieve planning- en ontwerpprojecten. De aanleiding van dit onderzoek komt voort uit de observatie dat landschappelijke plan- en ontwerpopgaven, met name in relatie tot klimaatadaptatie, om multifunctionele ruimtelijke oplossingen vragen. Daarbij is sprake van een toenemende vraag naar publieke participatie, wat zorgt voor een vergrote betrokkenheid van en diversiteit in wetenschappelijke experts, belanghebbenden en lokale bewoners bij ruimtelijke planning- en ontwerpprocessen. Om de communicatie tussen deze diverse groep betrokken belanghebbenden te faciliteren gebruiken planners en ontwerpers visuele representaties van ruimtelijke informatie, ideeën, en scenario's. Ondanks deze grote rol van beelden in het proces is er weinig onderzoek verricht naar hoe de verschillende vormen van visuele communicatie werken, wat daarvan de sociaalpolitieke effecten zijn, en hoe deze effecten de voortgang en uitkomsten van participatieve processen beïnvloeden.

Het doel van dit onderzoek is om bij te dragen aan een bewuster gebruik van visuele representaties binnen participatieve planning- en ontwerpprocessen door de visuele communicatie die gedurende dit soort projecten plaatsvindt te bestuderen als processen van betekenisgeving. Door de invloed van deze betekenisgevingsprocessen op de voortgang en uitkomsten van participatieve planning- en ontwerpprojecten te bestuderen kan visuele communicatie in de toekomst effectiever en transparanter plaatsvinden.

Het doel van dit onderzoek is vertaald in de volgende onderzoeksvraag:

Van wat voor soort processen van betekenisgeving maken visuele representaties van landschapsontwerpen deel uit, en hoe vormen deze processen de voortgang en uitkomsten van participatieve planning- en ontwerpprojecten? 
In dit proefschrift richt ik mij op het gebruik van visuele representaties van landschapsontwerpen als communicatiemiddel binnen participatieve planningen ontwerpprocessen van multifunctionele waterkerende landschappen. De voornaamste onderzoeksobjecten zijn dan ook (1) visuele representaties van landschapsontwerpen in alle mogelijke verschijningsvormen; (2) de betekenissen die aan deze representaties worden ontleent en toegekend door ontwerpers, planners, en belanghebbenden van een project; (3) de participatieve processen van de multifunctionele waterkerende landschapsprojecten waarbinnen deze visuele representaties zijn gemaakt en gebruikt. Om deze studie mogelijk te maken heb ik een zogeheten kritische visuele onderzoeksaanpak ontwikkeld door verschillende theorieën over betekenisgeving en visuele onderzoeksmethoden te verkennen en toe te passen.

De relatie tussen visuele representaties, hun maakprocessen en de interpretaties door derden zie ik in dit proefschrift als een proces van betekenisgeving. Dit betekenisgevingsproces heet in de literatuur semiosis. De bestudering van dit proces wordt ook wel de semiotiek, of betekenisleer, genoemd. Als object van betekenisgeving conceptualiseer ik visuele representaties van landschapsontwerpen, en hun invloed op participatieve processen, middels de theorie van Peirceaanse semiotiek. Volgens Peirce, een filosoof en semioticus, geven mensen betekenis aan de wereld middels een mediatie van tekens, zoals geluiden, geuren, kleuren, emoties, sentimenten. In principe kan alles een teken zijn, zolang het maar een bepaalde betekenis heeft voor iemand.

Op conceptueel niveau bevat een teken drie componenten: representamen, object en interpretant. Het representamen bestaat uit het deel van het teken dat wij kunnen waarnemen: een fysieke manifestatie als geur, vorm, of beeld. Het object bestaat uit datgene waar het teken naar verwijst. Bijvoorbeeld, een overstromingskaart (representamen) verwijst naar de mate waarin een bepaald gebied is overstroomd, of zou kunnen overstromen (object). De interpretant 
bestaat uit de interpretatie van het object door iemand, middels het waarnemen van het representamen. Deze interpretatie leidt vervolgens tot een handeling of invloed, wat ook wel de kritieke functie van tekens wordt genoemd. Dit drieledig perspectief op de semiotiek vormt het conceptuele raamwerk van dit proefschrift. Visuele representaties zijn tekens die zelf ook weer een grote hoeveelheid aan tekens bevatten, welke bestudeerd worden vanuit vier perspectieven: de kritieke functies, het representamen, de interpretant, en het object.

Literatuurstudie laat zien dat verschillende concepten uit de semiotiek en de kritische sociale theorie bruikbaar zijn voor het bestuderen van de manier waarop visuele representaties van landschapsontwerpen worden gebruikt. In mijn onderzoek bestudeer ik representaties in verschillende stadia van betekenisgeving met verschillende visuele onderzoeksmethoden, zoals een iconografische content analyse, een sociaal-semiotische analyse en een visuele discours analyse. Deze onderzoeksaanpak heeft mij in staat gesteld dominante machtsstructuren, miscommunicatie tussen betrokkenen, en het ontstaan van een visuele padafhankelijkheid in planning- en ontwerpprocessen te verklaren.

Deze voorgestelde kritische visuele onderzoeksaanpak heb ik toegepast op drie zogeheten stadia van visuele communicatie: het beeld, de ontvangst, en de productie daarvan. Deze stadia zijn gerelateerd aan de eerder genoemde componenten van het teken in de semiotiek, respectievelijk de representamen, de interpretant, en het object. Het stadium van het beeld is bestudeerd in termen van 'semiotische complexiteit'; het ontvangen van dat beeld is bestudeerd in termen van 'interpretatieve gewoonten'; het productie stadium is bestudeerd in termen van het 'visuele discours' dat in de afbeelding wordt besloten gedurende het maakproces.

Het bestuderen van de betekenisgevingsprocessen waar visuele representaties van landschapsontwerpen deel van uit maken heb ik gedaan middels een exploratieve case studie aanpak. De empirische case die representatief is voor het bestudeerde fenomeen is geïdentificeerd als de 'Rebuild by Design' 
ontwerpwedstrijd, specifiek de ontwerpprocessen die in het kader hiervan hebben plaatsgevonden, en de visuele representaties die daarbij zijn gemaakt. Rebuild by Design was een trans-disciplinaire ontwerpwedstrijd die plaatsvond in het grotere metropolitaanse gebied van New York na de overstromingen die veroorzaakt werden door de superorkaan Sandy in 2012. De visuele communicatie die tijdens deze ontwerpprocessen heeft plaatsgevonden heb ik bestudeerd aan de hand van beeldanalyse en interviews met de makers en het publiek van deze beelden.

De semiotische complexiteit van het beeld is bestudeerd aan de hand van een iconografische content analyse. Hierbij zijn 759 afbeeldingen geanalyseerd, welke geproduceerd zijn binnen de 'New Meadowlands' en 'Living with the Bay' prijsvraaginzendingen. Het gebruikte theoretische raamwerk maakt onderscheid tussen vier hoofdcategorieën waarmee de 'visuele inhoud' van een beeld gelezen, gemaakt, en bediscussieerd kan worden: medium, mode, formulering, en kennis. Deze categorieën stellen ons in staat de semiotische complexiteit van beelden te vatten in termen van validiteit, leesbaarheid, en interactiviteit. De validiteit van een ontwerp is gerelateerd aan de functionaliteit, visuele esthetiek, en haalbaarheid van het voorstel. In dit geval houdt dat in dat de voorgestelde oplossingen passend moeten zijn om tot een goed multifunctioneel waterkerend landschap te komen; of deze oplossingen gewenst en uitvoerbaar zijn; en of er voldoende draagvlak voor deze ideeën is onder de betrokkenen van het project. De leesbaarheid van een ontwerp bepaalt de mate waarin de deelnemers van het planning- en ontwerpproces de inhoud van het ontwerp kunnen lezen en begrijpen. De interactiviteit van een ontwerp omvat het medium waarop het wordt gepresenteerd en de mate waarin dat medium de ontvanger in staat stelt om de inhoud en manier van presentatie aan te passen. Wat precies valide, leesbaar of interactief is hangt af van zowel de interpretatieve gewoonten van de ontvanger als van de discursieve invloed van de makers van het beeld.

De interpretatieve gewoonten van de ontvangers zijn bestudeerd met behulp van een sociaal-semiotisch post-fundamenteel discours analytisch raamwerk (SSPDA). 
Dit raamwerk is toegepast op een gedeeltelijke reconstructie van de sociaalpolitieke context van drie Rebuild by Design projecten: het 'New Meadowlands' voorstel in New Jersey, het 'Resist, Delay, Store, Discharge' voorstel in Hoboken en het 'Big-U' project op Manhattan. Door interviews met de betrokkenen van deze projecten te analyseren heb ik contraproductieve interpretaties blootgelegd die, op het moment van beeldproductie, door de ontwerpers en planners hadden kunnen worden voorzien. Door dit soort conflicterende interpretatieve gewoonten te herkennen gedurende specifieke fases van het ontwerpproces kunnen planners en ontwerpers beter anticiperen op productieve en contraproductieve interpretaties van hun ontwerpen. Planners en ontwerpers kunnen vervolgens hun visuele representaties afstemmen op de interpretatieve gewoonten van hun publiek, of ze kunnen proberen die gewoonten te beïnvloeden door middel van de discursieve invloed van deze representaties.

Visueel discours zit ingebed in visuele representaties van landschapsontwerpen. Dit is bestudeerd door representaties te conceptualiseren als discursieve materialisaties van kennis en invloed. Deze conceptualisatie houdt in dat de sociale relaties waarbinnen de productie van representaties plaatsvind, en de manier waarop deze relaties zich manifesteren in specifieke communicatieve kwaliteiten van representaties, bestudeerd kunnen worden aan de hand van een visuele discours analyse. De analyse van de Rebuild by Design ontwerpwedstrijd liet een onderlinge afhankelijkheid zien tussen de manier waarop het participatieve proces is gearrangeerd, de interactiviteit van het medium dat wordt gebruikt en de visuele retoriek die voortkomt uit de visuele compositie van het beeld. Een bewuster gebruik van deze discursieve componenten door planners en ontwerpers helpt miscommunicatie te voorkomen, verwachtingen te managen en de validiteit van de uitkomsten van participatieve processen te verhogen.

Het antwoord op het eerste gedeelte van de onderzoeksvraag van dit proefschrift bestaat uit een getest analytisch raamwerk voor een kritische visuele 
onderzoeksaanpak. Volgens deze aanpak worden de betekenisgevingsprocessen van visuele representaties van landschapsontwerpen geconceptualiseerd als de relatie tussen semiotische complexiteit, interpretatieve gewoonten, en visueel discours. De semiotische complexiteit van het beeld leidt tot uiteenlopende gradaties van validiteit, leesbaarheid, en interactiviteit. Het in kaart brengen van de interpretatieve gewoonten van belanghebbenden helpt planners en ontwerpers te anticiperen hoe de validiteit, leesbaarheid, en interactiviteit geïnterpreteerd zal worden en tot welke reacties en handelingen dit zal leiden. Deze interpretatieve gewoonten worden continu beïnvloed door tekens, en systemen van tekens, met discursieve eigenschappen. De productie van visuele representaties wordt daarom geconceptualiseerd in termen van deze discursieve invloed; het zogeheten visuele discours.

Het antwoord op het tweede deel van de onderzoeksvraag van dit proefschrift is het resultaat van de toepassing van de kritische visuele onderzoeksaanpak op de empirische case study Rebuild by Design. De invloed van de betekenisgevingsprocessen van visuele representaties op de voortgang en uitkomsten van participatieve planning- en ontwerpprocessen wordt gecategoriseerd in een typologie van negen semiotische kwaliteiten. Deze typologie maakt gebruik van een combinatie van het drieledige onderscheid in communicatieve elementen (validiteit, leesbaarheid, en interactiviteit), en de drievoudige functies van tekens, te weten de representationele, existentiële, en symbolische functies.

Representationele functies behelzen de kwaliteiten en karakteristieken van een ontwerp; de mate waarin een representatie daadwerkelijk valide, leesbaar, en interactief is. Representationele validiteit omvat bijvoorbeeld de mate waarin het ontwerp een representatie is van de kennis, expertise, en voorkeuren van de participanten van een project, en of het ontwerp functioneel, esthetisch, en haalbaar is. Representationele leesbaarheid duidt op de mate waarin de kennis en ideeën die zijn bijgedragen door participanten ook gelezen en begrepen kan worden door 
die participanten. Representationele interactiviteit gaat in op de mate waarin de openheid van het gebruikte medium de participanten in staat stelt om hun kennis te delen en het beeld tot zich te nemen zoals zij dat willen. Denk hierbij aan het kunnen toevoegen van informatie, of het kunnen wijzigen van de schaal of het perspectief waarmee het beeld wordt weergegeven.

Symbolische ofwel de retorische en connotatieve kwaliteiten van een representatie bepalen of die representationele kwaliteiten daadwerkelijk aanwezig zijn, of dat ze slechts als zodanig worden ervaren. Symbolische validiteit, bijvoorbeeld, omvat de mate waarin de validiteit van een ontwerp wordt beïnvloed door de geloofwaardigheid en autoriteit van de maker, of door de ogenschijnlijke complexiteit van de ontworpen oplossing, in plaats van door de daadwerkelijke functionaliteit, esthetiek, en haalbaarheid van het ontwerp. Symbolische leesbaarheid duidt op een vergelijkbare subjectiviteit. Hier gaat het om de mate waarin de visuele stijl en retoriek van het beeld aansluit op of conflicteert met de interpretatieve gewoonten van de ontvanger. Niet alleen het kunnen lezen en begrijpen van het beeld is hier van belang, maar vooral de vraag of het beeld aan bepaalde visuele verwachtingen voldoet voert hier de boventoon. De symbolische interactiviteit omvat de mate waarin de openheid van het medium aansluit op het te verwachten participatieniveau in het planning- of ontwerpproces. Hierbij moet gedacht worden aan het organiseren van een interactieve tekensessie terwijl de hoofdlijnen voor het ontwerp eigenlijk al vastliggen, of aan de weergave op een passief projectiescherm waarmee alleen wat eerste ontwerpideeën worden gepresenteerd.

De existentiële kwaliteiten van visuele representaties zorgen ervoor dat de representationele en symbolische kwaliteiten van een ontwerp enerzijds te herleiden zijn tot beslissingen die eerder in het proces zijn gemaakt, en anderzijds bepaalde richtingen en denkkaders voor de toekomst bepalen. De existentiële validiteit omvat bijvoorbeeld het idee dat bepaalde partijen, zoals een gemeente of projectontwikkelaar, het ontwerp valide genoeg moeten vinden om een project daadwerkelijk te willen uitvoeren. Anderzijds kunnen andere partijen zoals 
belangenorganisaties juridische stappen ondernemen om het project stil te leggen omdat zij een ontwerp niet als valide zien. Existentiële leesbaarheid wordt bepaald door het wel of niet ontwikkelen van een gezamenlijke visuele taal gedurende het proces. Dit kan leiden tot efficiëntere communicatie, maar ook tot moeizamere communicatie wanneer nieuwe belanghebbenden die deze visuele taal niet machtig zijn, zoals een jurylid of wethouder, in een later stadium deel gaan uitmaken van het proces. Existentiële interactiviteit omvat het trainen van participanten in het tekenen van schetsen of maken van maquettes teneinde deze betrokkenen te activeren. Tegelijkertijd kan de interactiviteit van moderne technieken, zoals de 'maptable', een barrière vormen en daarmee belanghebbenden buitensluiten.

De kritische visuele onderzoeksaanpak, het analytische raamwerk, en de typologie van semiotische kwaliteiten zoals gepresenteerd in dit proefschrift bieden een handreiking voor het omgaan met de situationele complexiteit van participatieve planning- en ontwerpprocessen. Allereerst gaat het semiotische vocabulaire, zoals voorgesteld in de iconografische content analyse, verder dan alleen het categoriseren van inhoud of visuele technieken. Het gaat uit van het idee dat iedere kleur, tekentechniek, ieder perspectief, medium, of tekstueel element een teken an sich is. Elk van deze variabelen beïnvloedt, als teken dat onderhevig is aan semiosis, de interpretatie van het beeld als geheel en de inhoud van het ontwerp dat het beeld representeert. Ten tweede wordt in dit proefschrift benadrukt dat interpretaties geen 'black boxes' zijn, maar dat zij als interpretatieve gewoonten gevoed door een specifieke sociaal-politieke context wel degelijk te anticiperen zijn in termen van contingentie. Ten slotte wordt de concrete invloed die visuele representaties hebben op deze interpretatieve gewoonten verklaard vanuit het discursieve perspectief dat dit proefschrift inneemt. Visueel discours staat altijd in dienst van de ideologieën en typen kennis die er onderdeel van uitmaken. Visueel discours, als discursief concept, heeft als functie assimilatie met en dominantie over de sociaal-politieke context van een participatief planning- en ontwerpproces te bewerkstelligen. Als planner of ontwerper is het herkennen en erkennen van deze 
discursieve krachten essentieel voor het bewuster beïnvloeden van interpretatieve gewoonten teneinde een open en transparant communicatieproces te faciliteren.

In dit proefschrift stel ik voor op een nieuwe manier naar visuele representaties en daarmee ook naar participatieve processen van landschapsontwerpen te kijken, waarbij planners en ontwerpers als faciliterende actoren de meest passende communicatieve representatiestrategie kunnen bepalen op het juiste moment en voor het juiste publiek. Om tot een effectieve communicatie te komen moeten alle drie de varianten van validiteit, leesbaarheid, en interactiviteit in acht worden genomen. In de praktijk zal de hiërarchie tussen deze semiotische kwaliteiten afhangen van de ruimtelijke opgave, de betrokken belanghebbenden, en de manier waarop participatie wordt georganiseerd. Desalniettemin, een goed oog voor het verschil tussen representationele en symbolische kwaliteiten kan planners en ontwerpers helpen om feiten van fictie onderscheiden, een goed visueel narratief te herkennen, en door visuele cosmetische façades heen te prikken. Ook helpt het om, in het kader van participatie, effectieve media te gebruiken in plaats van moderne technologische snufjes. De existentiële kwaliteiten van representaties helpen te achterhalen waar bepaalde ideeën vandaan komen, welke visuele taal gewenst is, en hoe 'direct' betrokkenen kunnen worden benaderd. Tegelijkertijd dwingt het de planner of ontwerper ook om vooruit te kijken: welke paden slaan we hiermee in, hoe definitief zijn deze, en wat voor productieve en contraproductieve interpretaties kunnen we daarbij verwachten? 



\section{Acknowledgements}

The idea to pursue academic travels in hyper-reality was originally formed during a very fruitful year at the NCBR at the Radboud University. The NCBR's critical intellectual climate propagated by Henk van Houtum, as well as by my good friend Rodrigo Bueno Lacy, sparked in me the desire for theorizing towards a critical perspective on all visual depictions of geographic phenomena. The Landscape Architecture group at Wageningen University offered me the opportunity to further develop this critical perspective. I gratefully acknowledge the support, supervision, guidance, and open-mindedness of my daily supervisor Ingrid Duchhart. You helped me get acquainted with the (unwritten) theoretical traditions of a discipline that was largely unknown to me. My gratefulness extents, of course, to my co-promotor Wim van der Knaap, my promotor Adri van den Brink, and my external supervisor Gerda Roeleveld. Furthermore, I'd like to express my gratitude to all colleagues, friends, and family who supported and tolerated my philosophical ramblings over the years.

Finally but foremost: I dedicate this work to Renske, the love of my life, and to Simone, who waited graciously for this book to be submitted. 



\title{
SENSE
}

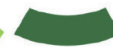

Netherlands Research School for the

Socio-Economic and Natural Sciences of the Environment

\section{I P L O M A}

For specialised PhD training

The Netherlands Research School for the Socio-Economic and Natural Sciences of the Environment (SENSE) declares that

\section{Kevin Michael Christian Raaphorst}

born on 11 February 1988 in Maarssen, the Netherlands

\author{
has successfully fulfilled all requirements of the \\ Educational Programme of SENSE.
}

Wageningen, 9 April 2019

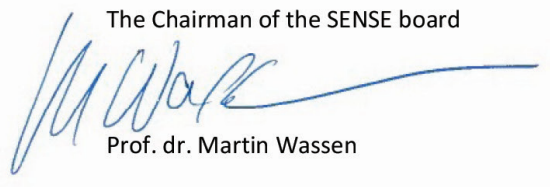

the SENSE Director of Education

The SENSE Research School has been accredited by the Royal Netherlands Academy of Arts and Sciences (KNAW)

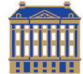

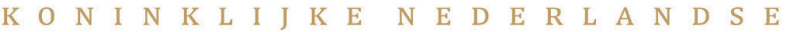

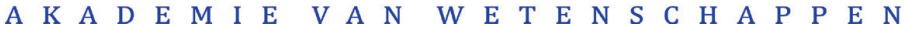




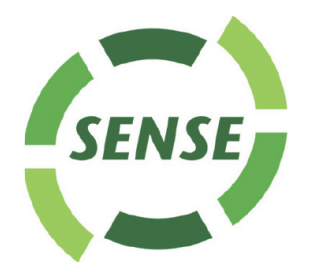

The SENSE Research School declares that Kevin Michael Christian Raaphorst has successfully fulfilled all requirements of the Educational PhD Programme of SENSE with a work load of $38.3 \mathrm{EC}$, including the following activities:

\section{$\underline{\text { SENSE PhD Courses }}$}

- Environmental research in context (2014)

- Research in context activity: 'Preparing, co-organizing, communicating and reporting in the wider context of $\mathrm{PhD}$ research in the form of reflection meeting, written contributions, general audience blogs and a research proposal' (2013-2017)

\section{Other PhD and Advanced MSc Courses}

- Interpretive Policy Analysis (IPA) pre-conference course, Wageningen School of Social Science (2014)

- Critical perspectives on social theory, Wageningen School of Social Science (2014)

- Scientific Writing, Wageningen Graduate Schools (2014)

- Communication with the media and the general public, Wageningen Graduate Schools (2016)

- Reviewing a scientific paper, Wageningen Graduate Schools (2016)

o Writing grant proposals, Wageningen Graduate Schools (2016-2017)

\section{Management and Didactic Skills Training}

- Supervising eight MSc students with thesis (2015-2018)

- Supervising $>20$ BSC student with thesis(2016-2018)

o Teaching in the BSc courses 'Introduction environmental sciences', 'Theory and methodology of planning and design', 'Research Methodology for human environment interactions', ' Landscape engineering' and 'Concepts and approaches in landscape architecture' (2014-2018)

- Teaching in the MSc courses 'Design theory' and 'Advanced design research methods' (2014-2017)

\section{Oral Presentations}

- The politics of design representations. Semiotic (un)predictability - IX Conference of Nordic Association for Semiotic Studies, 17-20 August 2015, Tartu, Estonia

- This too shall pass. Creation / Reaction - ECLAS Conference, 10-13 September 2017, Greenwich, United Kingdom

- Semiotic (un)predictability and the politics of landscape design representations. Sherlock Symposium - Sherlock Holmes and the semiotics of space and place, 16 March 2017, Wageningen, The Netherlands

\section{SENSE Coordinator PhD Education}

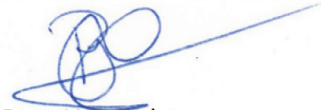

Dr. Peter Vermeulen 

The financial support for the research in this thesis by STW / TTW and Deltares is gratefully acknowledged.

\section{Colophon}

Cover design: Kevin Raaphorst, Monique Jansen

Lay-out: Monique Jansen (monique.jansen@wur.nl)

Printed by proefschiftmaken.nl

ISBN 978-94-6343-598-7

Copyright 2019 Kevin Raaphorst (kevin.raaphorst@wur.nl) 



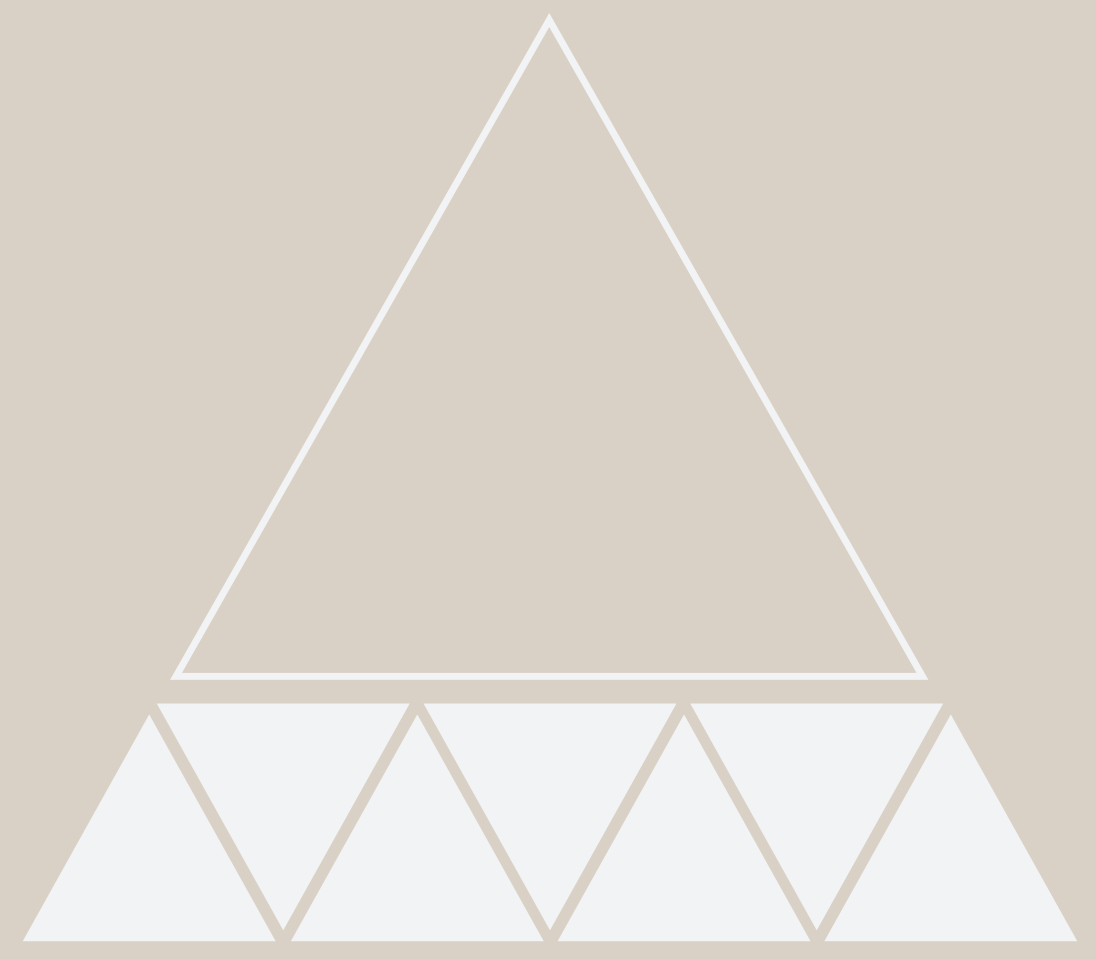

